\title{
Spektroskopie und Polarimetrie kleinskaliger magnetischer Strukturen der Sonnenoberfläche mit Methoden der Bildrekonstruktion
}

\author{
Dissertation \\ zur Erlangung des Doktorgrades \\ der Mathematisch-Naturwissenschaftlichen Fakultäten \\ der Georg-August-Universität zu Göttingen
}

\author{
vorgelegt von \\ Markus Koschinsky
}

aus

Bad Lauterberg

Göttingen 2001 
D 7

Referent: Prof. Dr. F. Kneer

Koreferent: Prof. Dr. D. Ronneberger

Tag der mündlichen Prüfung: 3. Mai 2001 


\section{Inhaltsverzeichnis}

$\begin{array}{ll}1 \text { Einleitung } & 5\end{array}$

$\begin{array}{lll}2 & \text { Einführung } & 7\end{array}$

2.1 Solare Magnetfelder . . . . . . . . . . . . . . . . . . . . . . 7

2.2 Messung solarer Magnetfelder $\ldots \ldots \ldots \ldots . \ldots \ldots$

$2.2 .1 \quad$ Zeeman-Effekt . . . . . . . . . . . . . . . . 10

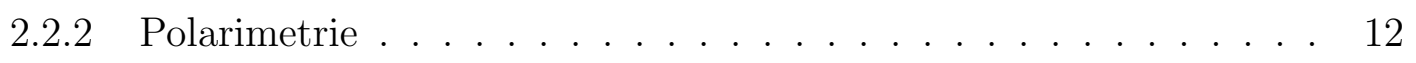

2.2 .3 Das Stokes-V-Polarimeter $\ldots \ldots \ldots$. . . . . . . . . . . 14

2.3 Seeing und Bildrekonstruktion $\ldots \ldots \ldots \ldots \ldots \ldots$

3 Optischer Aufbau 21

3.1 Das Vakuum-Turm-Teleskop (VTT) . . . . . . . . . . . . . . . . . 21

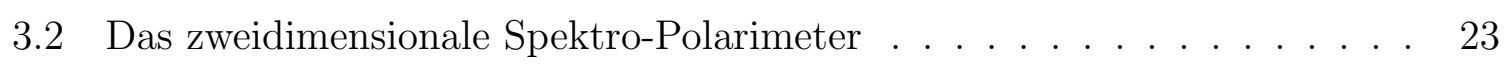

$3.2 .1 \quad$ Der Hauptstrahlengang $\ldots \ldots \ldots \ldots \ldots$

3.2 .2 Die Fabry-Perot-Interferometer (FPIs) $\ldots \ldots \ldots \ldots \ldots$

3.2 .3 Der Breitbandkana . . . . . . . . . . . . . . . . . . . 34

3.2 .4 Der Kontinuumsstrahlengang $\ldots \ldots \ldots \ldots \ldots$

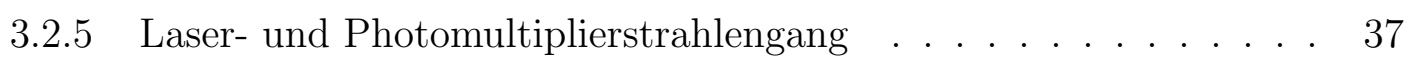

3.3 Instrumentelle Polarisation $\ldots \ldots \ldots \ldots \ldots . \ldots \ldots$

3.4 Der Beobachtungsablauf . . . . . . . . . . . . . . . . . . 40

4 Beobachtungen 42

$4.1 \quad$ Die Fe I-Linie bei $630,25 \mathrm{~nm} \ldots \ldots \ldots \ldots \ldots \ldots$

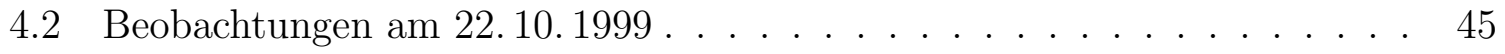

5 Datenverarbeitung 53

5.1 Bildrekonstruktion $\ldots \ldots \ldots \ldots \ldots \ldots \ldots \ldots \ldots \ldots$

$5.1 .1 \quad$ Vorverarbeitung der Daten . . . . . . . . . . . . . . . 54

5.1 .2 Korrektur der Bildverschiebung $\ldots \ldots \ldots \ldots \ldots$ 
5.1 .3 Frameselection . . . . . . . . . . . . . . . . . . 57

5.1.4 Aufteilen der Bilder in Teilbilder, Fouriertransformation und $\mathrm{Zu}-$ sammensetzen der Teilbilder . . . . . . . . . . . . . . . . . . . 59

5.1.5 Entfaltung mit der idealen Teleskopfunktion und Rauschfilterung 60

5.1 .6 Phase Diversity $\ldots \ldots \ldots \ldots \ldots \ldots$

5.1 .7 Speckle-Interferometrie . . . . . . . . . . . . . . . . . . 74

5.1 .8 Nachbearbeitung der Daten . . . . . . . . . . . . . . 80

5.1 .9 Vergleich der Rekonstruktionsmethoden … . . . . . . . . 81

5.2 Auswertung der spektroskopischen Information . . . . . . . . . . . . 85

$5.2 .1 \quad$ Korrektur der Wellenlängenverschiebung . . . . . . . . . . 85

5.2 .2 Korrektur der Filtertransmissionskurve . . . . . . . . . . . . 87

5.2 .3 Stokes-I- und-V-Spektren $\ldots \ldots \ldots \ldots \ldots \ldots$

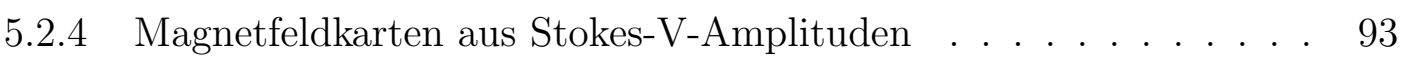

5.2 .5 Linienschwerpunktsmethode . . . . . . . . . . . . . . . . 94

$\begin{array}{lll}6 & \text { Ergebnisse } & 98\end{array}$

$6.1 \quad$ Ruhige Granulation $\ldots \ldots \ldots \ldots$

6.1.1 Gemischte Polaritäten innerhalb sehr kurzer Distanzen . . . . . . 101

6.1.2 Verstärkung von magnetischen Strukturen durch konvektiven Kollaps . . . . . . . . . . . . . . . . . . 103

$6.1 .3 \quad$ Zusammenhang zwischen magnetischem Fluss und Intensität . . . 105

6.1 .4 Zusammenhang zwischen magnetischem Fluss und Strömungen. . 108

6.1 .5 Dynamik und Lebensdauer kleiner Strukturen . . . . . . . . . 108

$6.1 .6 \quad$ Größe und magnetischer Fluss typischer Strukturen . . . . . . . . 113

6.2 Aktive Region (Plage $\ldots \ldots \ldots \ldots \ldots$

$6.2 .1 \quad$ Größe magnetischer Strukturen $\ldots \ldots \ldots$. . . . . . . . . 117

6.2 .2 Dynamik und Lebensdauer magnetischer Strukturen. . . . . . . . 120

6.2 .3 Zusammenhang zwischen magnetischem Fluss und Intensität . . . 120

6.2 .4 Zusammenhang zwischen magnetischem Fluss und Strömungen. . 126

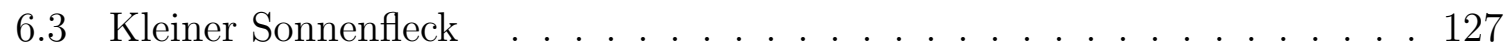

$\begin{array}{lll}7 & \text { Zusammenfassung und Ausblick } & 133\end{array}$

7.1 Einbau eines zweiten FPIs in das Spektro-Polarimeter . . . . . . . . . . 133

7.2 Bildrekonstruktion und Phase Diversity Methode . . . . . . . . . . . . 135

8.3 Untersuchung magnetischer Strukturen $\ldots \ldots \ldots \ldots$

\begin{tabular}{ll}
\hline Literaturverzeichnis & 141
\end{tabular} 


\section{Einleitung}

Die Sonne ist ein typischer Hauptreihenstern. Ihre Nähe macht sie zu einem idealen astronomischen Forschungsobjekt, wenn es um das Studium von stellaren Oberflächenstrukturen geht. Die Photosphäre der Sonne zeigt eine Vielzahl kleinskaliger Phänomene, deren Abmessungen von einigen 100 km oder weniger im Größenbereich der Skalenhöhe der Dichte liegen. Insbesondere in den aktiven Gebieten bestimmen Magnetfelder entscheidend die Struktur und Dynamik des Plasmas auf kleinen Skalen. Als typische magnetische Phänomene werden Flecken, Poren und Fackelpunkte beobachtet. Doch auch auf der nicht aktiven „ruhigen“ Sonnenoberfläche, die durch das Phänomen der Granulation geprägt ist, finden sich kleinskalige Strukturen, die Magnetfelder aufweisen.

Mit zunehmend besserer räumlicher Auflösung stellte sich heraus, dass, abgesehen von Sonnenflecken, das Magnetfeld der Sonne vorwiegend in kleinen Gebieten konzentriert zu sein scheint. Mit indirekten Methoden werden Flussdichten von bis zu 0,2 Tesla gemessen. Der Durchmesser der untersuchten Gebiete beträgt dabei nur wenige $100 \mathrm{~km}$. Verschiedene theoretische Modelle magnetischer Strukturen und numerische Simulationen sogenannter Flussröhren und ihrer Interaktion mit dem umgebenden Gas im Rahmen von Magnetohydrodynamik und Strahlungstransport zeigen komplexe, aber typische Strukturen und eine hohe Dynamik. Die Überprüfung und Verifizierung dieser Modelle macht Beobachtungen mit hoher räumlicher Auflösung unabdingbar, bei denen außerdem spektroskopische Informationen gesammelt werden müssen. Sowohl Methoden der zweidimensionalen Spektroskopie wie auch der Bildrekonstruktion zur Überwindung der störenden Einflüsse der Erdatmosphäre, die die räumliche Auflösung der Beobachtungen begrenzen, sind etablierte Arbeitsweisen der modernen Sonnenphysik.

Es war deshalb naheliegend, diese verschiedenen Ansätze zu kombinieren, um spektroskopische Information über zweidimensionale Bildfelder zu gewinnen, bei gleichzeitig höchster räumlicher Auflösung. Diesem Projekt gewidmet ist die Beobachtungsanlage „2d-Spektro-Polarimeter" der Arbeitsgruppe Sonnenphysik der Göttinger Sternwarte. Die technischen, personellen, finanziellen und zeitlichen Anforderungen an ein solches Projekt sind hoch. Deshalb befassen sich schon seit Jahren verschiedene Forschungsarbeiten mit der schrittweisen Annäherung an das Ziel, die wahren Strukturen der Sonnenoberfläche sichtbar zu machen und dabei aus der spektroskopischen Information die nötigen physikalischen Größen abzuleiten, die zum Verständnis der Vorgänge auf und in der Sonne nötig sind. 


\section{Einleitung}

Die vorliegende Arbeit begreift sich als Teil dieses Projekts. Schwerpunkte der Arbeit liegen in der technischen Weiterentwicklung und Umsetzung optischer Verbesserungen des 2d-Spektro-Polarimeters auf der einen Seite, und Entwicklung und Anwendung von Bildrekonstruktionsmethoden auf spektroskopische Daten von geringem Signal-zu-RauschVerhältnis auf der anderen Seite. Anhand magnetisch sensitiver Spektrallinien sollten Beobachtungsdaten aufgenommen und mit den angesprochenen Methoden verarbeitet werden. Die Ergebnisse sollen Aufschlüsse über die Struktur und Dynamik kleinster magnetischer Gebiete auf der Sonnenoberfläche geben.

Die Arbeit beginnt mit einer Einführung in den aktuellen Stand der Forschung über solare Magnetfelder, in die Prinzipien der Polarimetrie als Messmethode solarer Magnetfelder und in die Grundlagen der atmosphärischen Störeinflüsse und der Bildrekonstruktion. Es folgen Darstellungen des Beobachtungsaufbaus und der Datenaufnahme, an die sich eine genauere Beschreibung der Datenverarbeitung anschließt. Im Kapitel über Ergebnisse wird versucht, aus dem physikalischen Gehalt der Beobachtungsdaten neue Erkenntnisse über den Aufbau und die Funktionsweise magnetischer Strukturen zu gewinnen. Abschließend werden die Ergebnisse bewertet und ein Ausblick in folgende mögliche Entwicklungen der Beobachtungstechnik gegeben.

Die beobachtungstechnischen Aspekte und die Datenauswertung sind recht ausführlich und konkret beschrieben. Die nur an den sonnenphysikalischen Ergebnissen interessierte LeserIn mag viele Details ohne Schaden für das Verständnis der Arbeit überblättern. Für diejenigen aber, die selbst ähnliche Beobachtungen durchführen möchten, kann die Arbeit als Leitfaden dienen; außerdem soll sie den Blick dafür schärfen, dass eine gewissenhafte und sorgfältige Beobachtungstechnik und Datenverarbeitung die Grundlage aller zuverlässigen physikalischen Interpretationen und Ergebnisse sind. 


\section{Einführung}

\subsection{Solare Magnetfelder}

Der erste Nachweis, dass Sonnenflecken der Sitz starker Magnetfelder sind, gelang 1908 George E. Hale mit Hilfe der 12 Jahre zuvor von Pieter Zeeman entdeckten Aufspaltung bestimmter Spektrallinien im Magnetfeld. Mit immer besser aufösenden Instrumenten wurden räumlich immer kleinskaligere Magnetfelder entdeckt (vgl. u. a. Sheeley (1967), Beckers \& Schröter (1968), Keller \& v. d. Lühe (1992)]). Auch heute geht man davon aus, dass der wahre Durchmesser der einzelnen sog. Flussröhren unterhalb dem von mit heutigen Teleskopen direkt nachweisbaren Strukturen (ca. $150 \mathrm{~km}$ ) liegt, und dass alle größeren magnetischen Strukturen sich aus solchen kleinen Elementen zusammensetzen. Manche Modelle gehen sogar von typischen Größen von wenigen Kilometern aus, also weit unterhalb der Druckskalenhöhe und auch weit unterhalb der freien Weglänge der Photonen in der Sonnenatmosphäre (Sánchez Almeida et al. (1996), Sánchez Almeida \& Lites (2000) ). Obwohl sich diese Strukturen bisher der direkten Beobachtung und Bestimmung ihrer typischen physikalischen Parameter entziehen, ließen sich bis heute eine ganze Reihe von Aussagen über ihren Aufbau und ihre Natur machen.

Von der beobachtenden Seite her konnte gezeigt werden, dass die stärksten magnetischen Flussdichten von $300 \mathrm{mT}$ in den Umbren von Sonnenflecken auftreten. Schwächere Magnetfelder von $50 \mathrm{mT}$ in sog. Intra-Netzwerk-Elementen konnten mit Hilfe der „magnetic line ratio" Methode (Stenflo (1973) nachgewiesen werden (Lin (1995), Keller et al. (1994)). Lin \& Rimmele (1999) sprechen von „granularen Magnetfeldern“, bei denen sie magnetische Flussdichten bis herunter zu 5-10 mT aus der Zeeman-Aufspaltung von Spektrallinien im nahen Infrarot bestimmen. Messungen unter Ausnutzung des Hanle-Effekts geben sogar Hinweise auf ein turbulentes Hintergrundfeld mit Flussdichten von der Größenordnung 1 mT (Faurobert-Scholl (1993), Bianda et al. (1998), Bianda et al. (1998a), Stenflo et al. (1998)).

Verschiedene Beobachtungen weisen darauf hin, dass es zwei typische Varianten solarer Magnetfelder gibt. Zum einen die erwähnte schwache Feldkomponente mit 5-100 mT Flussdichte, die in ruhigen, nicht aktiven Gebieten auf der Sonne vorkommt, aber auch in aktiven Gebieten, den sog. Plages. Messungen des kompletten Stokes-Vektors (vgl. Kap. 2.2.2) mit anschließender Inversion der Stokes-Profile (Martínez Pillet et al. (1997)) zeigen für diese Felder vorwiegend geringe Füllfaktoren $\alpha \approx 0,1-0,2$ und starke Inklinationen $\gamma \approx 50^{\circ}-90^{\circ}$. Der Füllfaktor bezeichnet die mit magnetischem Fluss durchsetzten 


\section{Einführung}

Flächenanteile der Sonnenoberfläche, und die Inklination ist der Winkel zwischen den magnetischen Feldlinien und der Vertikalen auf der Sonnenoberfläche. Aus Gründen, die später erläutert werden sollen, sind diese Felder mit der verwendeten Beobachtungsanlage nur sehr schwer nachzuweisen, deshalb werden in der vorliegenden Arbeit vorwiegend die sog. „starken Felder“ untersucht. Diese treten u. a. an den Rändern der Supergranulen auf („Netzwerk“) und machen sich dort z. B. durch Aufhellungen im Linienkern der Spektrallinie CaII K bemerkbar (Soltau (1997)]), vor allen Dingen aber in den aktiven Gebieten, den „Plages“. Dort treten sie u. a. in Form von Poren, Fackelpunkten und „gestörter" Granulation auf. In Abb. 2.1 sind diese Phänomene deutlich zu erkennen. Typische Flussdichten dieser Strukturen sind $140 \mathrm{mT}$ auf Photosphärenniveau, die Füllfaktoren sind $\alpha \approx 0,1-0,7$, und die Inklination $\gamma$ ist in den meisten Fällen weniger als $10^{\circ}$, d. h. die Feldlinien stehen beinahe senkrecht auf der Sonnenoberfläche (Martínez Pillet et al. (1997), Soltau (1997)). Die einzelnen Stokes-Profile dieser magnetischen Strukturen zeigen typische Asymmetrien, die Hinweise auf Gasströmungen und die räumlich nicht aufgelöste, innere Struktur dieser Elemente und ihrer Umgebung geben (Martínez Pillet et al. (1997), Sigwarth (1999)).

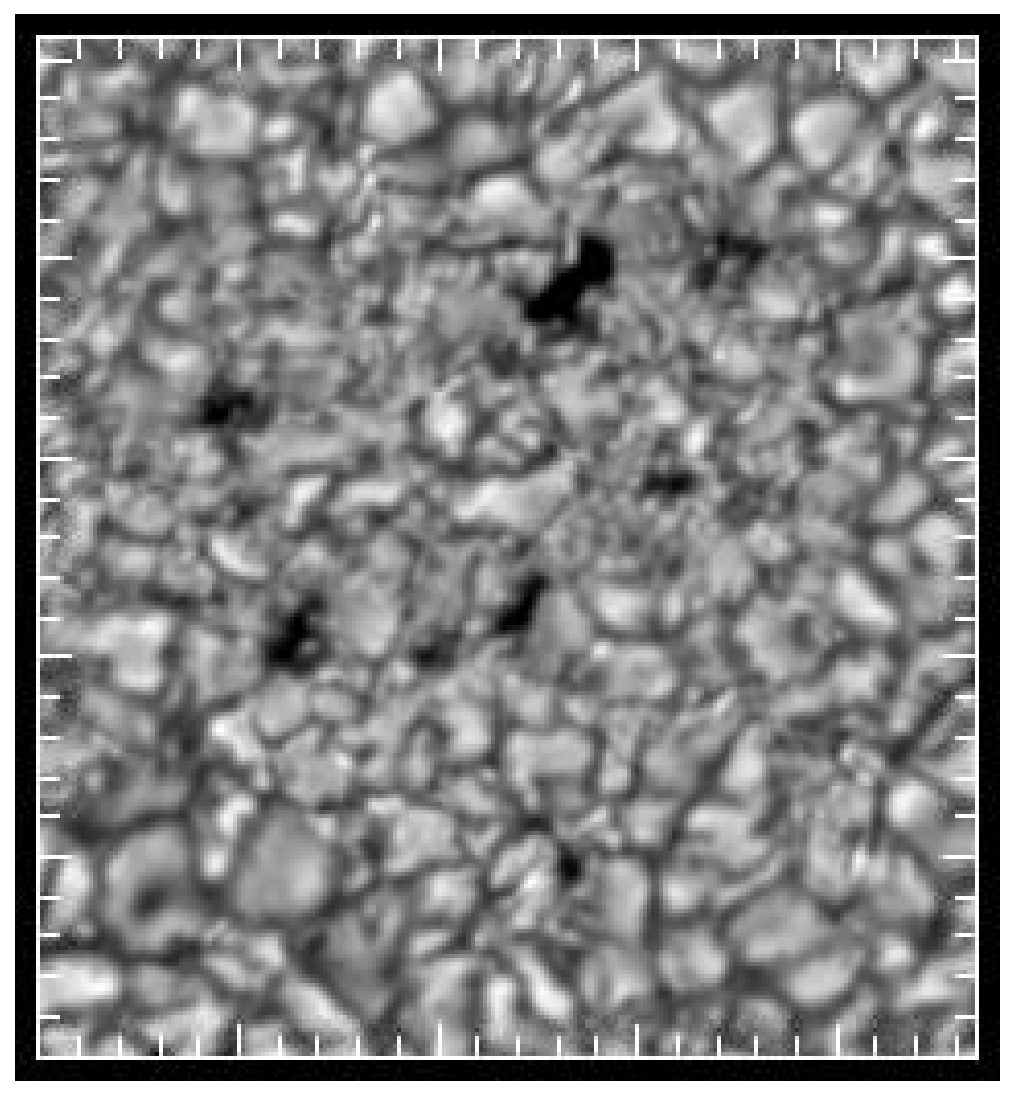

Abbildung 2.1: Diese Bildrekonstruktion (Speckle-Interferometrie) eines aktiven Gebiets (Plage-Region) vom 22.10.99 zeigt typische magnetische Strukturen: Poren, Fackelpunkte („Filigree"), gestörte Granulation. Der Abstand der Strichmarken entspricht 1". 
Von theoretischer Seite her stellt man sich vor, dass die magnetischen Feldlinien nicht gleichmäßig auf der Sonnenoberfläche verteilt sind, sondern sich in sog. Flussröhren oder Flussschläuchen konzentrieren, die in eine weitgehend feldfreie Umgebung eingebettet sind. Die Entstehung einer solchen starken Flussröhre kann durch das Zusammenwirken von zwei Prozessen beschrieben werden: Zunächst werden die im leitfähigen Plasma „eingefrorenen“ Feldlinien durch konvektive Strömungen aus dem Inneren der Granulen verdrängt und im intergranularen Raum konzentriert. Die Quelle dieser Magnetfelder kann das bereits erwähnte turbulente, schwache Magnetfeld sein, das den Raum zwischen den konzentrierten Flussröhren ausfüllt. Durch diese passive Konzentration kann das Feld ungefähr bis auf die Höhe der Äquipartitionsfeldstärke $B_{e}$ verstärkt werden. Đ Diese ist in der Photosphäre bei etwa $B_{e}=50 \mathrm{mT}$ erreicht, wenn die magnetische Energiedichte gleich der kinetischen Energiedichte der Gasströmung ist. Im zweiten Schritt tritt nun eine konvektive Instabilität ein, der „konvektive Kollaps“: Die angestiegene Feldstärke führt zu einer Verringerung des horizontalen konvektiven Energietransports, so dass das Gas im Bereich der Feldkonzentration radiativ abkühlt und adiabatisch in der superadiabatischen Umgebung absinkt. Dabei wird dieses Gas im Vergleich zu seiner Umgebung noch kälter, was die Abströmung beschleunigt. Dies führt zu einer teilweisen Evakuierung der Flussröhre. Um den fehlenden Gasdruck im Inneren der Röhre auszugleichen, muss diese in radialer Richtung kollabieren und so die Feldstärke erhöhen. Die Flussröhre ist nun ein Gebilde, bei dem der Gasdruck $p_{a}$ in der weitgehend feldfreien Umgebung im Gleichgewicht steht mit der Summe des Gasdrucks $p_{i}$ im Inneren und des magnetischen Drucks:

$$
p_{a}=p_{i}+\frac{B^{2}}{2 \mu} .
$$

Grundlagen hierzu und Modellrechnungen, auch zum dynamischen Verhalten dieser Flussröhren bei Interaktion mit der umgebenden Granulation, finden sich bei Stix (1989), Steiner et al. (1998), Grossmann-Doerth et al. (1998) und anderen. Durch die Annahme bestimmter Szenarien in Modellen lassen sich Asymmetrien von Linienprofilen auf unterschiedliche Weise erklären (Sánchez Almeida et al. (1996), Sánchez Almeida \& Lites (2000), Steiner (1999), Steiner (2000), u. a.). Dabei werden verschiedene Flussröhren mit bestimmten intrinsischen physikalischen Parametern (Feldstärke, Durchmesser, Polarität, etc.) in unterschiedliche Modelle nicht (oder auch schwach) magnetischer Atmosphären eingebettet, die wiederum durch den Verlauf bestimmter physikalischer Parameter mit der Höhe charakterisiert sind (Druck, Temperatur, Opazitäten, Geschwindigkeiten).

\footnotetext{
${ }^{1}$ Wie häufig in der Astrophysik wird hier die magnetische Flussdichte $B$ als magnetische Feldstärke

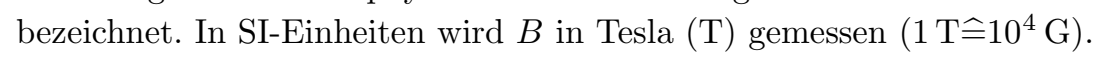




\subsection{Messung solarer Magnetfelder}

Magnetfeldmessungen auf der Sonne beruhen i. W. auf der Untersuchung geeigneter Spektrallinien unter Ausnutzung des Zeeman-Effekts oder des Hanle-Effekts. Letzterer wird meistens zur Messung sehr schwacher Magnetfelder verwendet und spielt daher bei den für diese Arbeit durchgeführten Untersuchungen keine Rolle.

\subsubsection{Zeeman-Effekt}

Die Anwesenheit eines Magnetfelds in einem Licht emittierenden Medium stellt eine Vorzugsrichtung dar und bewirkt die Aufhebung der Entartung der atomaren Energieniveaus. Solare Magnetfelder sind schwach in dem Sinne, dass die $L-S$-Kopplung von Bahndrehimpuls und Spin erhalten bleibt. Die entarteten Energieniveaus spalten daher in $2 J+1$ Unterniveaus auf. Die Energiedifferenz der aufgespaltenen Niveaus bzgl. der Energie ohne Magnetfeld ist

$$
\Delta E=g_{\mathrm{L}} \mu_{\mathrm{B}} M_{J} B
$$

mit $\mu_{\mathrm{B}}=0,927 \mathrm{Am}^{2}, M_{J}$ der Magnetquantenzahl des betreffenden Zustands und $g_{\mathrm{L}}$ dem Landé-Faktor, für den gilt:

$$
g_{\mathrm{L}}=1+\frac{J(J+1)+S(S+1)-L(L+1)}{2 J(J+1)} .
$$

Dabei sind $L, S$ und $J$ die Bahn-, Spin- und Gesamtdrehimpulse des betreffenden Atom-

${ }^{5} \mathbf{D}_{\mathbf{0}} \quad \mathbf{g}_{\mathrm{L}}=\mathbf{0}$

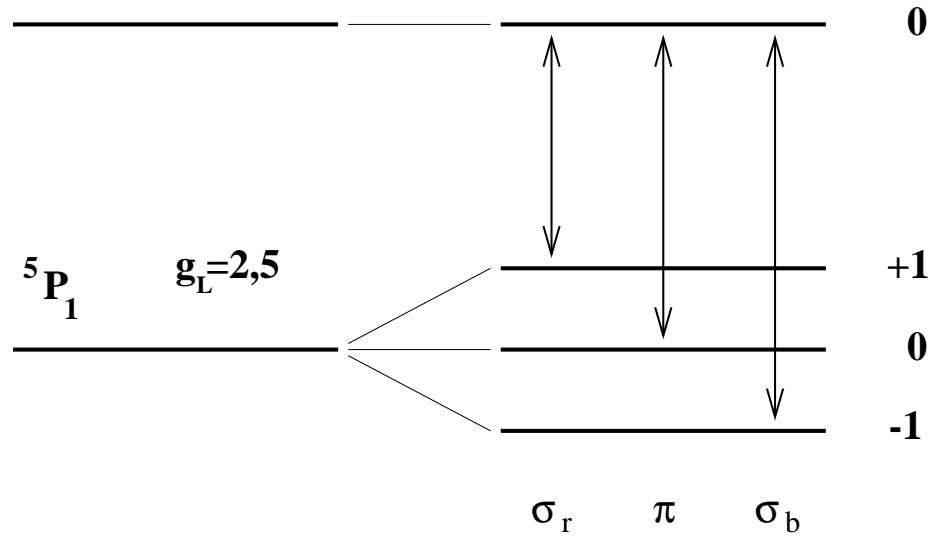

Abbildung 2.2: Termschema für die zu einem normalen Zeeman-Triplett aufgespaltene Linie Fe I 630,25 nm. Das obere Niveau ${ }^{5} D_{0}$ spaltet wegen $J=0$ nicht auf. zustands. Im allgemeinen Fall $\mathbf{M}_{\mathbf{J}} \quad$ spalten beide Energieniveaus, die an dem bei einer Lichtemission bzw. Absorption stattfindenden Übergang beteiligt sind, mit verschiedenen LandéFaktoren auf. Dadurch ergibt sich aus den einzelnen, nach den entsprechenden Auswahlregeln möglichen Übergängen ein komplexes Aufspaltungsmuster der Spektrallinie. Man spricht in diesem quantenmechanischen Allgemeinfall vom „anomalen Zeeman-Effekt". Die Übergänge mit $\Delta M_{J}=0$ ergeben die sog. $\pi$-Komponenten, die Komponenten, für die $\Delta M_{J}= \pm 1$ gilt, werden $\sigma$-Komponenten genannt. 
Für den Fall, dass beide beteiligten Energieniveaus denselben Landé-Faktor und damit dieselbe Aufspaltung haben oder dass eines der Niveaus wegen $J=0$ keine Aufspaltung im Magnetfeld zeigt, ergeben sich jeweils eine $\pi$-Komponente und zwei $\sigma$-Komponenten. Man spricht dann von einem „normalen Zeeman-Triplett“ (Abb. 2.2). Die Magnetfeldstärke ergibt sich aus der beobachteten Aufspaltung $\Delta \lambda_{B}$ der $\sigma$-Komponenten gegenüber der $\pi$-Komponente:

$$
B=\frac{4 \pi c m_{\mathrm{e}}}{e} \frac{\Delta \lambda_{B}}{\lambda_{0}^{2} \cdot g_{\mathrm{eff}}} .
$$

Hierbei bezeichnen $c$ die Lichtgeschwindigkeit, $m_{\mathrm{e}}$ die Elektronenmasse, $e$ die Elementarladung und $\lambda_{0}$ die Ruhewellenlänge der Spektrallinie ohne Magnetfeld, alle Angaben gelten im SI-System. $g_{\text {eff }}$ wird effektiver Landé-Faktor genannt und ist im Fall des normalen Zeeman-Effekts gerade $g_{\mathrm{L}}$. Im allgemeinen Fall des anomalen Zeeman-Effekts ergibt sich zunächst für jede einzelne Zeeman-Komponente ein Landé-Faktor $g_{\text {Komp }}$ aus den Landé-Faktoren und den Magnetquantenzahlen der beiden an dem Übergang beteiligten Energieniveaus:

$$
g_{\text {Komp }}=g_{L} M_{J}-g_{L}^{\prime} M_{J}^{\prime} .
$$

Der effektive Landé-Faktor $g_{\text {eff }}$ ergibt sich dann aus einer Mittelung aller Einzellinien der $\sigma$ - bzw. der $\pi$-Komponente; dabei werden die unterschiedlichen Dipolmomente und damit die Linienstärken der beteiligten Übergänge durch entsprechende Gewichtung bei der Mittelwertbildung berücksichtigt.

Im sichtbaren Spektralbereich ist die Linienaufspaltung für Feldstärken unterhalb etwa $150 \mathrm{mT}$ gering im Vergleich zur Dopplerbreite, so dass die Zeeman-Aufspaltung nicht direkt gemessen werden kann. Auch bei höheren Feldstärken kann die Aufspaltung schwer zu beobachten sein, wenn nur ein Bruchteil der beobachteten Sonnenoberfläche eines Aufösungselements von den Magnetfeldern durchsetzt wird. In beiden Fällen kann man sich aber hilfreich der Polarimetrie bedienen. Grundlage der Messmethode ist, dass die einzelnen Zeeman-Komponenten verschiedene Polarisationszustände haben. Bei transversaler Beobachtung, wenn die Sichtlinie senkrecht zu den magnetischen Feldlinien steht, sind die $\sigma$-Komponenten

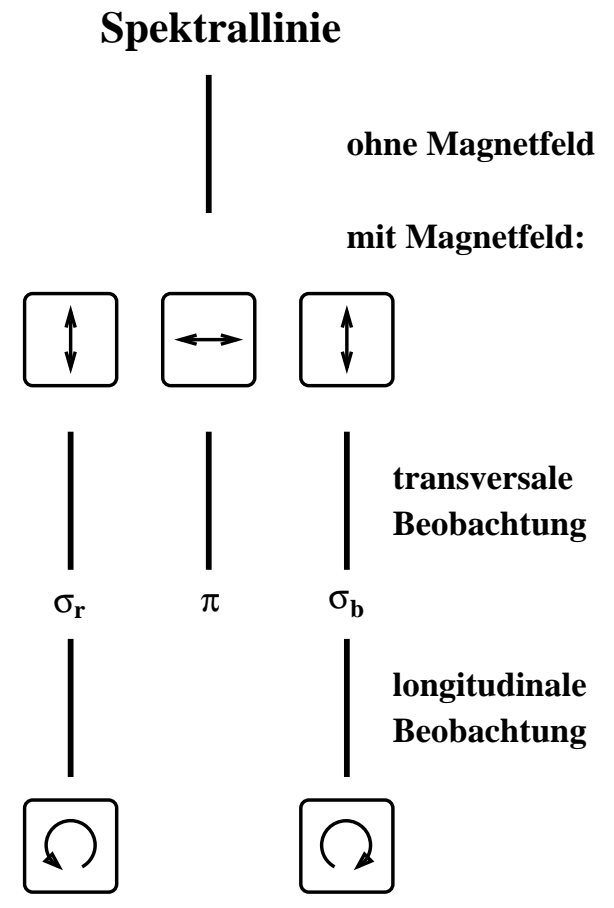

Abbildung 2.3: Polarisation beim Zeeman-Effekt senkrecht linear polarisiert zu der ebenfalls linear polarisierten $\pi$-Komponente. Bei Messung der Spektrallinien mit Sichtlinie parallel zu den Feldlinien (longitudinaler Fall) verschwindet die $\pi$-Komponente und die $\sigma$ - 
Komponenten sind, je nach Polarität des magnetischen Felds, rechts- bzw. linkszirkular polarisiert (Abb. 2.3). Ist die Sichtlinie in einem beliebigen Winkel zu den Feldlinien geneigt, ergeben sich Polarisation und Stärke der einzelnen Komponenten aus der Addition der entsprechenden Anteile des Magnetfelds senkrecht bzw. parallel zur Sichtlinie. Weiterhin ist bei der Messung von Absorptionslinien zu beachten, dass nicht die eigentlichen, an dem Übergang im Atom beteiligten Lichtquanten gemessen werden, sondern Restintensitäten nicht absorbierten Lichts. Entsprechend polarisierte Anteile des Lichts mit jeweils $90^{\circ}$ gedrehten Polarisationsrichtungen erreichen die BeobachterIn. Man spricht in diesem Fall vom „inversen Zeeman-Effekt“.

\subsubsection{Polarimetrie}

Die von der Wellenlänge abhängige Polarisation einer Spektrallinie kann auch im Fall nicht aufgespaltener Linienprofile gemessen werden, wozu geeignete Messgeräte, sog. "Polarimeter" benutzt werden. Zunächst ist es aber sinnvoll, eine geeignete mathematische Beschreibung polarisierten Lichts zu geben. Das Intensitätsprofil $I(\lambda)$ einer magnetischen Spektrallinie setzt sich i. Allg. aus einem Anteil unpolarisierter $I_{0}(\lambda)$ und elliptisch polarisierter Strahlung $I_{\mathrm{p}}(\lambda)$ zusammen. Die vier "Stokes-Parameter" oder "Stokes-Profile“ $I(\lambda), Q(\lambda), U(\lambda)$ und $V(\lambda)$ ergeben zusammen den "Stokes-Vektor" $\vec{S}(\lambda)$. Er beschreibt den Polarisationszustand des Lichts mit Hilfe der polarimetrisch messbaren Intensitäten:

$$
\vec{S}(\lambda)=\left\{\begin{array}{c}
I \\
Q \\
U \\
V
\end{array}\right\}(\lambda):=\left\{\begin{array}{c}
I_{0}+I_{\mathrm{p}} \\
I_{\operatorname{lin}}\left(0^{\circ}\right)-I_{\mathrm{lin}}\left(90^{\circ}\right) \\
I_{\operatorname{lin}}\left(45^{\circ}\right)-I_{\mathrm{lin}}\left(135^{\circ}\right) \\
I_{\text {zirk }}^{+}-I_{\text {zirk }}^{-}
\end{array}\right\}(\lambda)
$$

so dass gilt:

$$
I_{p}^{2}(\lambda)=Q^{2}(\lambda)+U^{2}(\lambda)+V^{2}(\lambda) .
$$

$I_{\text {zirk }}^{+}$und $I_{\text {zirk }}^{-}$bezeichnen rechts- bzw. linkszirkulare Polarisation, $I_{\text {lin }}(\theta)$ die lineare Polarisation in der Polarisationsebene $\theta$. Diese wird in geeigneter Weise bzgl. des Beobachtungsaufbaus festgelegt und enthält noch eine Unbestimmtheit von $180^{\circ}$, die sich auch auf die Festlegung des Azimuts der Feldrichtung bzgl. der Sichtlinie überträgt.

Eine mathematisch sauberere Definition des Stokes-Vektors stützt sich auf die Beschreibung des Wellencharakters von Licht mittels des elektrischen Feldvektors $\vec{E}$. Gegeben sei ein einzelner, monochromatischer elektromagnetischer Wellenzug, der sich in $z$-Richtung ausbreitet. Dann schwingt der elektrische Feldvektor in der $x-y$-Ebene:

$$
E_{x}=\xi_{x} \cos \phi, \quad E_{y}=\xi_{y} \cos (\phi+\epsilon)
$$

mit $\phi=\omega t-k z$ und $\epsilon$ der Phasendifferenz zwischen $E_{x}$ und $E_{y}$ mit den Amplituden $\xi_{x}$ und $\xi_{y}$. Dann sind die Stokes-Parameter folgendermaßen definiert (Shurcliff (1962)): 


$$
\begin{array}{ll}
I=\xi_{x}^{2}+\xi_{y}^{2} & Q=\xi_{x}^{2}-\xi_{y}^{2} \\
U=2 \xi_{x} \xi_{y} \cos \epsilon & V=2 \xi_{x} \xi_{y} \sin \epsilon
\end{array}
$$

Für nicht streng monochromatische Wellenpakete und teilweise polarisiertes Licht sind entsprechend zeitliche Mittelwerte dieser Größen einzusetzen.

Die Stokes-Profile erhält man durch Differenzbildung von Spektren, in denen zuvor senkrecht zueinander stehende Polarisationsrichtungen getrennt wurden. Das klassische Stokes-V-Polarimeter z. B. zerlegt das einfallende Licht in die beiden Teilstrahlen $\left[\frac{1}{2}\left(I_{0}+\right.\right.$ $\left.\left.I_{\text {lin }}\right)+I_{\text {zirk }}^{+}\right]$und $\left[\frac{1}{2}\left(I_{0}+I_{\text {lin }}\right)+I_{\text {zirk }}^{-}\right]$. Nach der spektralen Zerlegung ergibt sich aus der Differenz $\left[I_{\text {zirk }}^{+}(\lambda)-I_{\text {zirk }}^{-}(\lambda)\right]$, welches das Stokes-V-Profil $V(\lambda)$ ist. Die Summe $\left[I_{0}+I_{\text {lin }}+\right.$ $\left.I_{\text {zirk }}^{+}(\lambda)+I_{\text {zirk }}^{-}(\lambda)\right]$ ist das Stokes-I-Profil $I(\lambda)$. Kürzer lassen sich die beiden polarimetrischen Kanäle auch durch die Ausdrücke $\frac{1}{2}\left(I_{\lambda}+V_{\lambda}\right)$ bzw. $\frac{1}{2}\left(I_{\lambda}-V_{\lambda}\right)$ charakterisieren. Auf analoge Weise können mit Polarimetern, die senkrecht zueinander linear polarisiertes Licht trennen, Stokes-Q- und -U-Profile gemessen werden.

Die genaue Bestimmung von $\vec{B}$ (Betrag und Richtung des Feldvektors auf der Sonne) bedarf der Messung und Analyse des gesamten Stokes-Vektors $\vec{S}(\lambda)$. Im Folgenden werden kurz die Gründe erläutert, warum in der vorliegenden Arbeit lediglich Stokes-V-Profile verwendet werden:

- Selbst bei einer Inklination von $\gamma=45^{\circ}$, bei der gleichermaßen der longitudinale wie auch der transversale Zeeman-Effekt auftreten, ist das auf die Kontinuumsintensität $I_{\mathrm{c}}$ normierte Stokes-V-Profil stärker als die entsprechenden Q- und UProfile. Das hat sowohl atomphysikalische wie auch sonnenphysikalische Gründe: Zum einen hängt die Stärke des Stokes-V-Signals linear von dem Verhältnis von Zeeman-Aufspaltung zu Dopplerbreite der Linie $\Delta \lambda_{B} / \Delta \lambda_{\text {Doppler }}$ ab, die Stärke der Q- und U-Signale dagegen quadratisch. Da das Verhältnis für die meisten Felder und die Spektrallinien im sichtbaren Bereich kleiner als eins ist, werden intrinsisch stärkere V-Signale erzeugt (Landi Degl'Innocenti (1992)). Hinzu kommt, dass die Magnetfelder innerhalb eines Auflösungselements auf der Sonne bei ähnlichen Inklinationen verschiedene Azimute aufweisen können, z. B. wenn man in eine sich aufweitende Flussröhre hineinschaut. Solange die longitudinale Feldkomponente immer dasselbe Vorzeichen hat, summieren sich die Stokes-V-Signale auf. StokesQ- und -U-Signale können dagegen bei verschiedenen Azimuts unterschiedliche Vorzeichen haben und sich deshalb gegenseitig auslöschen. Dies führt zu einer weiteren Verringerung der ohnehin schon schwächeren Q- und U-Signale.

- Um alle vier Stokes-Parameter möglichst gleichzeitig mit kurzen Belichtungszeiten und kurzer Gesamtdauer für die Aufnahme eines kompletten Datensatzes zu messen, wäre ein hoher instrumenteller Aufwand nötig, der mit der vorhandenen Beobachtungsanlage nicht geleistet werden konnte. 
- Die linearen Stokes-Parameter sind, wenn sie denn gemessen werden, physikalisch schwerer zu interpretieren als Stokes-V-Signale. Dies liegt u. a. daran, dass die genaue Zuordnung der gemessenen Polarisationsrichtung bzgl. des Winkels zwischen der Beobachtungsrichtung und der Sonnenoberfläche kompliziert ist. Bestimmte Auswertemethoden wie die Linienschwerpunktsmethode (vgl. Kap. 5.2.5) sind nur beim Stokes-V-Signal anwendbar.

- Die instrumentelle Polarisation durch schräge Reflexionen an den Coelostatenspiegeln des Teleskops und weiteren Umlenkspiegeln betrifft hauptsächlich die linear polarisierten Anteile. Dadurch würden hierbei die systematischen Messfehler vergrößert (vgl. Kap. 3.3).

Wegen der Beschränkung der Messungen auf die zirkulare Stokes-Komponente wurden als Beobachtungsobjekte magnetische Strukturen ungefähr in der Scheibenmitte ausgewählt. Da die meisten Flussröhren in magnetisch stärkeren Strukturen nur geringe Inklinationen $\gamma \lesssim 10^{\circ}$ aufweisen (Martínez Pillet et al. (1997)), ist für solche Beobachtungen ein größtmögliches Stokes-V-Signal zu erwarten, und weitgehend kein Q- oder U-Signal, auf dessen Messung deshalb auch guten Gewissens verzichtet werden darf.

\subsubsection{Das Stokes-V-Polarimeter}

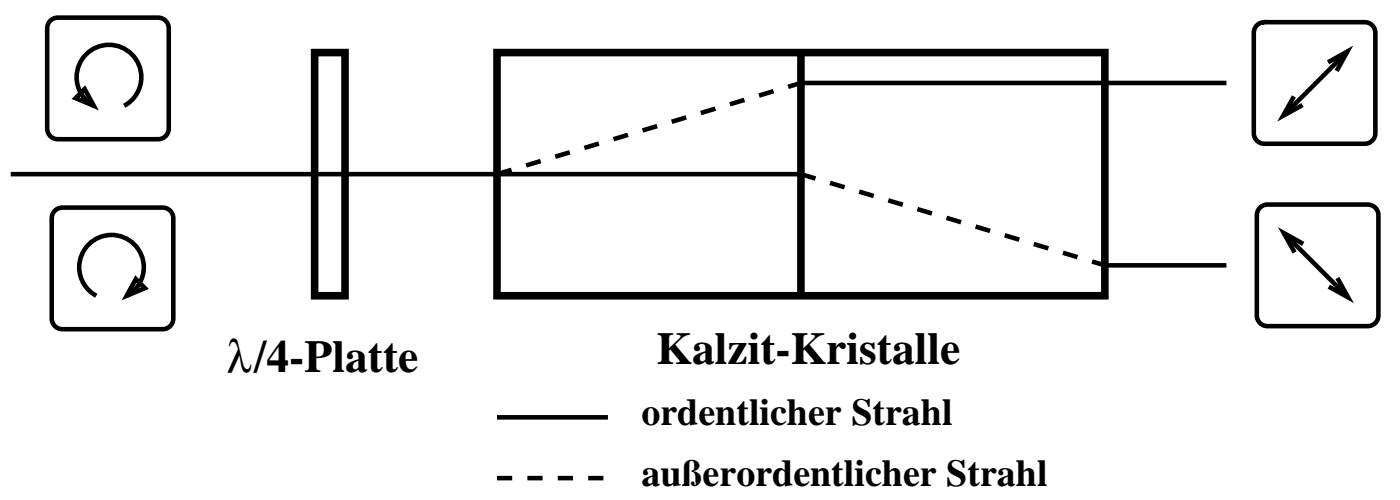

Abbildung 2.4: Aufbau des Stokes-V-Polarimeters

Das in dieser Arbeit verwendete Polarimeter zur Trennung der rechts- bzw. linkszirkular polarisierten Zeeman-Komponenten ist ein klassisches Stokes-V-Polarimeter, bestehend aus einer $\lambda / 4$-Verzögerungsplatte und zwei doppelbrechenden Kalzitkristallen. Die Verzögerungsplatte wandelt zunächst rechts- bzw. linkszirkular polarisiertes Licht in zwei senkrecht zueinander stehende linear polarisierte Lichtsorten um. Dahinter sind nacheinander zwei Kalzitkristalle so angeordnet, dass diese beiden Teilstrahlen linear polarisierten Lichts räumlich voneinander getrennt werden, so dass sie nebeneinander auf dem Detektor auftreffen. Dabei wird darauf geachtet, dass der Teilstrahl, der den 
ersten Kristall als ordentlicher Strahl durchläuft, im zweiten Kristall der außerordentliche Strahl ist und umgekehrt. So ist sichergestellt, dass die beiden Teilstrahlen durch das Polarimeter insgesamt gleiche optische Weglängen haben und das Polarimeter parallel gegeneinander versetzt verlassen (Abb. 2.4). Die Aufspaltung des Lichtbündels ist gerade so groß, dass beide Bildfelder nebeneinander auf einem CCD-Chip abgebildet werden können. Eine genauere Beschreibung des verwendeten Polarimeters findet sich bei $\operatorname{Volkmer~(1995),~}$

Die Wirkung des Polarimeters lässt sich auch mathematisch mit Hilfe von Stokes-Vektoren und Muellermatrizen ausdrücken (Shurcliff (1962), Koschinsky (1995)]). Eine Muellermatrix ist eine $4 \times 4$-Matrix, welche die polarimetrischen Eigenschaften eines optischen Geräts vollständig beschreibt. Dieses Gerät verändert die Polarisation eines Lichtstrahls in folgender Weise:

$$
\vec{S}^{\prime}=M \cdot \vec{S}
$$

Dabei seien $\vec{S}, \vec{S}^{\prime}$ die Stokes-Vektoren des Lichts vor bzw. nach Durchgang durch das Gerät und $M$ dessen Muellermatrix. Der Durchgang durch mehrere verschiedene optische Komponenten lässt sich leicht durch mehrfache Matrizenmultiplikation beschreiben. Die Wirkung einer $\lambda / 4$-Verzögerungsplatte, deren Vorzugsrichtung („schnelle Achse“) parallel zu einer frei gewählten Referenzrichtung des optischen Aufbaus steht, und ihre Wirkung auf zirkular polarisiertes Licht lässt sich in diesem Formalismus durch folgende Muellermatrix beschreiben:

$$
\vec{S}^{\prime}=\left(\begin{array}{cccc}
1 & 0 & 0 & 0 \\
0 & 1 & 0 & 0 \\
0 & 0 & 0 & 1 \\
0 & 0 & -1 & 0
\end{array}\right)_{\lambda / 4} \cdot\left(\begin{array}{l}
1 \\
0 \\
0 \\
1
\end{array}\right)=\left(\begin{array}{l}
1 \\
0 \\
1 \\
0
\end{array}\right)
$$

Rechtszirkular polarisiertes Licht wird also vollständig in linear polarisiertes umgewandelt, dessen Polarisationsrichtung einen Winkel von $45^{\circ}$ zur Referenzrichtung hat. Entsprechend wird linkszirkular polarisiertes Licht nach Durchgang durch eine $\lambda / 4$-Verzögerungsplatte linear polarisiert sein und einen Winkel von $135^{\circ}$ zur Referenzrichtung haben. Die darauf folgenden Kalzitkristalle trennen das Licht räumlich in zwei Teilstrahlen, für die jeweils eine eigene Muellermatrix angegeben werden muss. Die Kristalle sind in geeigneter Orientierung so hinter der $\lambda / 4$-Verzögerungsplatte angeordnet, dass entsprechend ihren anisotropen optischen Eigenschaften $M_{\text {Kalzit, } 1}$ und $M_{\text {Kalzit, } 2}$ die Muellermatrizen von Linearpolarisatoren unter einem Winkel von $\pm 45^{\circ}$ sind:

$$
M_{\text {Kalzit, } 1}=\frac{1}{2}\left(\begin{array}{cccc}
1 & 0 & 1 & 0 \\
0 & 0 & 0 & 0 \\
1 & 0 & 1 & 0 \\
0 & 0 & 0 & 0
\end{array}\right) \quad \text { bzw. } \quad M_{\text {Kalzit, } 2}=\frac{1}{2}\left(\begin{array}{cccc}
1 & 0 & -1 & 0 \\
0 & 0 & 0 & 0 \\
-1 & 0 & 1 & 0 \\
0 & 0 & 0 & 0
\end{array}\right)
$$

Daraus ergeben sich die beiden Muellermatrizen des Polarimeters für die beiden Teil- 
strahlen:

$$
M_{\text {Polar, 1, 2 }}=M_{\text {Kalzit , 1, }} \cdot M_{\lambda / 4}=\frac{1}{2}\left(\begin{array}{cccc}
1 & 0 & 0 & \pm 1 \\
0 & 0 & 0 & 0 \\
\pm 1 & 0 & 0 & 1 \\
0 & 0 & 0 & 0
\end{array}\right)
$$

wobei das + jeweils für den einen Teilstrahl und das - für den anderen gelten soll. Betrachten wir nun die Wirkung des Polarimeters auf vollständig zirkular polarisiertes Licht $\vec{S}_{\text {zirk }}=(I, Q, U, V)^{T}=(1,0,0,1)^{T}$ :

$$
\vec{S}_{\text {zirk }, 1,2}^{\prime}=M_{\text {Polar }, 1,2} \cdot \vec{S}_{\text {zirk }}=\frac{1}{2}\left(\begin{array}{cccc}
1 & 0 & 0 & \pm 1 \\
0 & 0 & 0 & 0 \\
\pm 1 & 0 & 0 & 1 \\
0 & 0 & 0 & 0
\end{array}\right) \cdot\left(\begin{array}{l}
1 \\
0 \\
0 \\
1
\end{array}\right)=\frac{1}{2}\left(\begin{array}{c}
1 \pm 1 \\
0 \\
\pm 1+1 \\
0
\end{array}\right)
$$

Solches Licht würde also vollständig in den einen Teilstrahl übertragen werden und diesen als linear polarisiertes Licht verlassen. Am Ausgang des anderen Kanals würde die Intensität null gemessen. Licht entgegengesetzter Polarisationsrichtung $\vec{S}_{\text {zirk }}=(I, Q, U, V)^{T}=$ $(1,0,0,-1)^{T}$ würde vollständig in den anderen Kanal übertragen werden. Unpolarisiertes Licht $\vec{S}_{0}=\left(I_{0}, 0,0,0\right)^{T}$ und linear polarisiertes Licht $\vec{S}_{\text {lin }}=\left(\sqrt{Q^{2}+U^{2}}, Q, U, 0\right)^{T}$ dagegen werden gleichmäßig auf beide Teilstrahlen verteilt:

$$
\begin{aligned}
\vec{S}_{0+\text { lin }, 1,2}^{\prime} & =M_{\text {Polar, } 1,2} \cdot \vec{S}_{0+\text { lin }}=\frac{1}{2}\left(\begin{array}{cccc}
1 & 0 & 0 & \pm 1 \\
0 & 0 & 0 & 0 \\
\pm 1 & 0 & 0 & 1 \\
0 & 0 & 0 & 0
\end{array}\right) \cdot\left(\begin{array}{c}
I_{0}+\sqrt{Q^{2}+U^{2}} \\
Q \\
U \\
0
\end{array}\right) \\
& =\frac{1}{2}\left(\begin{array}{c}
I_{0}+\sqrt{Q^{2}+U^{2}} \\
0 \\
\pm\left(I_{0}+\sqrt{Q^{2}+U^{2}}\right) \\
0
\end{array}\right)
\end{aligned}
$$

Beim Bilden des Differenzsignals der beiden Kanäle fallen also tatsächlich alle unpolarisierten und linear polarisierten Anteile des Lichts heraus, und nur die rechts- bzw. linkszirkular polarisierten Anteile bleiben übrig. Dagegen enthält die Summe der Kanäle sämtliches einfallende Licht, es bildet das Stokes-I-Signal.

\subsection{Seeing und Bildrekonstruktion}

Mit „Seeing“ werden im Allgemeinen die Einflüsse der Erdatmosphäre auf astronomische Beobachtungen bezeichnet. Dieses sind unerwünschte Störeinflüsse, deren Auswirkungen möglichst gering gehalten werden sollen. Das kann auf der einen Seite durch geeignete Maßnahmen bei der Datengewinnung erreicht werden, auf der anderen Seite nachträglich 
durch Bildverarbeitung und Bildrekonstruktion. Im Folgenden soll eine kurze, qualitative Einführung in das Wesen des Seeings und in die Grundlagen der Bildrekonstruktion gegeben werden. Da eine ausführliche Beschreibung der einzelnen Methoden der Bildverarbeitung auch in der Anwendung auf konkrete Datensätze gezeigt werden soll, erfolgt die detaillierte Erklärung der Methoden erst später in Kap. 5.1.

Ursache des Seeings sind lokale Temperaturschwankungen in der Atmosphäre. Diese bewirken Dichteschwankungen und damit Fluktuationen des Brechungsindexes, die das von der Sonne kommende Licht beeinflussen. Obwohl diese Fluktuationen gering sind, können sich die Störungen durch den langen Lichtweg in der Erdatmosphäre aufaddieren und so die Abbildung im Teleskop beeinträchtigen.

Der auf den ersten Blick am deutlichsten sichtbare Effekt ist ein ständig wechselnder, seitlicher Bildversatz („image motion“). Dieser kann durch Kreuzkorrelationsmethoden beseitigt werden, indem nachträglich die Bilder ,aufeinandergeschoben“ werden. Weiterhin wird aber eine Vielzahl von zeitlich und räumlich mehr oder weniger schnell veränderlichen Abbildungsfehlern erzeugt. Grobe Bildverzerrungen treten als lokale Änderungen des Bildmaßstabs auf, quasi durch Linseneffekte von Turbulenzelementen. Diesen ist durch Zerlegen der großen Bildfelder in Teilbilder in gewissem Maße beizukommen. Dabei werden aus unterschiedlich verzerrten Einzelbildern die sich entsprechenden, gegeneinander verschobenen Teilgebiete ausgeschnitten und wieder zusammengesetzt. Sogenannte „destretching Algorithmen“ können diese Aufgabe automatisieren.

Weiterhin treten Unschärfen auf, durch die die feinen Strukturen des beobachteten Objekts teilweise bis zur Unkenntlichkeit verschmiert und verändert werden, obwohl sie von dem Teleskop bis knapp an seine theoretische Auflösungsgrenze übertragen werden sollten. Zu diesen Abbildungsfehlern gehören zu einem wesentlichen Teil ein Vor- und Zurücklaufen des Fokus in der theoretischen Fokalebene des Teleskops um mehrere Wellenfronten $\Gamma$, aber auch seitliche Verzerrungen in einer Vorzugsrichtung (Astigmatismus, Koma), Verdopplungen der Bilder usw. Natürlich tritt auch jede Form von Kombinationen dieser Fehler auf, so dass sich ein zeitlich sehr variables, komplexes „Verzerrungsmuster" ergibt. Als Folge dessen unterscheiden sich sämtliche einzeln aufgenommenen Bilder ein und desselben Objekts: Sie zeigen unterschiedlich viele Feinstrukturen (was sich anhand des Powerspektrums nachweisen lässt), und diese sehen in jedem Bild noch etwas unterschiedlich aus.

Mathematisch lässt sich der Einfluss des Seeings anhand der Punktverbreiterungsfunktion (Point Spread Function, PSF) oder deren Fouriertransformierten, der optischen Übertragungs- oder Transferfunktion (OTF) beschreiben. Die Punktverbreiterungsfunktion ist gerade die Intensitätsverteilung des Bilds, das ein abbildendes System von einer punktförmigen Intensität $I=I_{0} \delta\left(x-x_{0}, y-y_{0}\right)$ eines Objekts in der Abbildungsebene entwirft. Für ein flächenhaftes Objekt beschreibt sie, wie das abbildende System die Intensität, die von jedem einzelnen Punkt des Objekts ausgeht und von dem System detektiert wird, über die Bildebene verteilt. Bezeichnen wir im Folgenden mit $o(\vec{x})$ ein 
perfektes Abbild des beobachteten Objekts. Das mit einem realen abbildenden System beobachtete Bild $i(\vec{x})$ des Objekts ergibt sich dann gerade aus einer Faltung des perfekten Abbilds mit der PSF $s(\vec{x})$ des Systems:

$$
i(\vec{x})=o(\vec{x}) * s(\vec{x})+n(\vec{x})
$$

$n$ sei hierbei Rauschen, das in realen Bildern immer auftritt. Die Faltung entspricht im Fourierraum dem Produkt der Fouriertransformierten. Gehen wir davon aus, dass der Informationsgehalt einer Funktion und seiner Fouriertransformierten gleich ist? und bezeichnen wir im Folgenden mit Kleinbuchstaben Größen im Ortsraum als Funktion der Koordinaten $\vec{x}$ und mit Großbuchstaben deren Fouriertransformierte als Funktionen der Frequenzkoordinaten $\vec{s}$. Glg. 2.16 wird dann zu

$$
I(\vec{s})=O(\vec{s}) \cdot S(\vec{s})+N(\vec{s})
$$

Die Fouriertransformierte $S(\vec{s})$ der PSF ist die optische Transferfunktion OTF und beschreibt, mit welcher Intensität Strukturen einer bestimmten Größe, die gerade einer Raumfrequenz $\vec{s}$ entsprechen, von dem abbildenden System übertragen werden. Beispiele von PSFs und OTFs werden in Abb. 5.3 und 5.9 gegeben.

Aufgabe der Bildrekonstruktion ist es nun, folgende Frage zu lösen: Wie sieht das beobachtete Objekt, von dem eine bestimmte Anzahl unterschiedlicher Aufnahmen vorliegen, wirklich aus? Grundlage aller Bildrekonstruktionsmethoden ist, aus den verschiedenen Einzelaufnahmen möglichst viele vertrauenswürdige Informationen über das "wahre“ Objekt herauszuziehen und aus diesen gesammelten Informationen ein Bild dieses Objekts herzustellen, das dessen reale Eigenschaften so genau wie möglich darstellt. Die Grenzen der Genauigkeit werden im Wesentlichen durch zwei Faktoren gegeben: Zum einen durch die Leistungsfähigkeit des optischen Systems, mit dem die Einzelaufnahmen erhalten werden. Dabei gehören zum optischen System die Atmosphäre, das Teleskop und die Postfokusinstrumentierung. Je mehr Informationen von diesem System übertragen werden, um so mehr Information kann in dem rekonstrierten Bild enthalten sein. Zum anderen können Informationen, die in den Einzelbildern nur sehr schwach enthalten sind, von Rauschen überdeckt werden. Rauschquelle ist in erster Linie das poissonverteilte Photonenrauschen des Lichts selbst, also das Eintreffen verschieden vieler Photonen in gleichen Zeiträumen aufgrund des statistischen atomphysikalischen Prozesses der Lichtemission. Aber auch das Ausleseverhalten des CCD-Chips und Ungenauigkeiten in der weiteren, elektronischen Verarbeitung der Daten sind Rauschquellen, die zudem noch unterschiedliche und teilweise unbekannte statistische Verhalten aufweisen können.

Die erwähnten Beschränkungen in der Übertragung von Informationen über Strukturen bestimmter Größen, die von der OTF $S(\vec{s})$ des abbildenden Systems beschrieben werden, lassen sich genauer betrachten. Die offensichtlichste Einschränkung ist natürlich

\footnotetext{
${ }^{2}$ Diese Annahme ist nicht per se erfüllt. Beispielsweise existiert von einem diskreten Datenfeld, welches einen linearen Gradienten enthält, keine gleichwertige Darstellung im Fourierraum.
} 


\subsection{Seeing und Bildrekonstruktion}

das Auflösungsvermögen des Teleskops, welches durch seinen Durchmesser D und die Wellenlänge $\lambda$ des beobachteten Lichts gegeben ist. Die OTF eines idealen Teleskops ist in Abb. 5.3 (S. 61) aufgetragen, sie beginnt bei Raumfrequenz null mit einem Wert von 1, was gerade eine Transmission des Geräts von $100 \%$ bedeutet. Sie fällt dann kontinuierlich bis auf null ab bei der Grenzfrequenz $s_{\text {grenz }}$, die gerade dem Auflösungsvermögen des Teleskops entspricht:

$$
A=\lambda / D
$$

Das bedeutet, dass Strukturen des betrachteten Objekts, die größer sind als $1 / s_{\text {grenz }}$, zwar von dem Teleskop übertragen werden, aber ihr Kontrast, je nach dem Wert der OTF, vermindert wird. Dies findet Ausdruck in dem Unterschied zwischen dem Powerspektrum des Objekts und dem der gewonnenen Abbildung. Bei einem („realen“) Teleskop, das Abbildungsfehler aufweist, liegt die OTF immer unterhalb der idealen OTF, kann lokale Maxima und Minima, Nulldurchgänge und negative Werte aufweisen.

Die Abbildungsfehler, die durch das Seeing hervorgerufen werden, ergeben ebenfalls eine OTF, die die Übertragungsqualität der Atmosphäre für Strukturen bestimmter Größen beschreibt. Diese ist im Groben der OTF eines Teleskops ähnlich. Sie kann ebenfalls z. B. lokale Maxima oder Minima aufweisen, vor allen Dingen ist sie zeitlich variabel, man spricht deshalb auch häufig von einer „momentanen OTF“. Eine für die Bildrekonstruktion wesentliche Eigenschaft der atmosphärischen OTF wurde von Labeyrie (1970) beschrieben. Er stellte fest, dass die atmosphärische OTF (bzw. deren Betragsquadrat) von kurzbelichteten Aufnahmen bis zu sehr kleinen Strukturen im Mittel größer als null ist. Kurzbelichtet heißt hier, dass sich die OTF der Atmosphäre während der Belichtungszeit nicht oder nicht wesentlich ändern soll, man spricht auch von „eingefrorenem Seeing“. Eben diese Tatsache eröffnet der Bildrekonstruktion die Möglichkeit, Informationen über sehr kleine Strukturen des Objekts, bis fast hinunter zur Beugungsgrenze $1 / s_{\text {grenz }}$ des Teleskops, aus einzelnen, kurzbelichteten Aufnahmen zu entnehmen, die erst bei langbelichteten Aufnahmen durch die Überlagerung vieler einzelner kurzbelichteter Aufnahmen ,verschmiert" werden und verloren gehen. Aus dem Verhältnis des mittleren Powerspektrums von kurzbelichteten Aufnahmen und dem Powerspektrum einer gemittelten, quasi langzeitbelichteten Aufnahme lässt sich ein Seeingparameter (Fried (1966)) abschätzen, mit dessen Hilfe anhand der Theorie der atmosphärischen Turbulenz Aussagen über die grobe, mittlere Form der atmosphärischen OTF gemacht werden kann. Dieses Verfahren wird „Methode des spektralen Quotienten“ (bzw. engl. „spectral ratio method") genannt (v. d. Lühe (1984), Korff (1973)).

Letztlich kann natürlich auch die Postfokusinstrumentierung bestimmte Abbildungsfehler enthalten und die Informationen über das beobachtete Objekt je nach Größe der Strukturen entsprechend seiner OTF schwächen oder gar ganz verloren gehen lassen.

Die OTF des gesamten optischen Systems ergibt sich aus dem Produkt der OTFs der einzelnen Komponenten, eine Unterscheidung dieser OTF-Komponenten ist aber zunächst nicht einfach und im Rahmen der verschiedenen Bildrekonstruktionsmetho- 


\section{Einführung}

den auch nicht unbedingt nötig. Natürlich fällt eine erfolgreiche Bildrekonstruktion am leichtesten, wenn die Gesamt-OTF über einen möglichst großen Bereich von Raumfrequenzen möglichst hohe Werte hat, folglich schon in den beobachteten Einzelbildern ein möglichst hoher Informationsgehalt über die Strukturen verschiedenster Größe des Objekts besteht. Die OTFs der Komponenten des abbildenden Systems lassen sich einzeln optimieren. Zunächst wird man ein Teleskop von möglichst großer Eintrittsöffnung und guter optischer Qualität benutzen. Beim verwendeten VTT (Vakuum-Turm-Teleskop) am Observatorio del Teide auf Teneriffa ergibt sich aus dem Durchmesser von $70 \mathrm{~cm}$ bei einer Wellenlänge von $\lambda=630.25 \mathrm{~nm}$ ein Auflösungsvermögen von 0,186" , was einer Größe der Strukturen auf der Sonnenoberfläche von $135 \mathrm{~km}$ entspricht\$. Außerdem wird man für das Teleskop einen geeigneten Standort und gute Beobachtungsbedingungen (Uhrzeit, Wetter) wählen, so dass das Seeing besonders gut ist. Dies ist am VTT aufgrund der geografischen Gegebenheiten des Standorts, der Konstruktion und Ausführung des Gebäudes (z. B. große Höhe der Eintrittsöffnung über dem sich erwärmenden Erdboden, weißer Anstrich aller umliegenden Gebäudeteile, Evakuierung großer Teile des Teleskops) häufig in den Morgenstunden gegeben, so dass dann für einen großen Teil der kurzbelichteten Einzelaufnahmen die OTF bis zur Auflösungsgrenze des Teleskops deutlich größer als null ist. Letztlich kann der Anteil der Postfokusinstrumentierung an der Gesamt-OTF minimiert werden. Hier kann durch große Sorgfalt bei der Gestaltung des optischen Aufbaus, der Auswahl der einzelnen Bauteile und deren Justierung erreicht werden, dass dieser Anteil an der gesamten OTF weitgehend vernachlässigt werden kann.

Die verschiedenen Methoden der Bildrekonstruktion unterscheiden sich in dem Verfahren, wie die einzelnen Anteile der Gesamt-OTF bestimmt werden und welche dieser Anteile bei der Bildung des rekonstruierten Bilds korrigiert werden. Unterschiedlich sind dementsprechend die technischen Anforderungen an das benötigte Datenmaterial und die Fehleranfälligkeit und letztlich natürlich der Erfolg der Rekonstruktion, also der Informationsgehalt der rekonstruierten Bilder über die wahren Strukturen verschiedener Größe des beobachteten Objekts.

\footnotetext{
${ }^{3} 1^{\prime \prime} \widehat{\approx} 725 \mathrm{~km}$ auf der Sonnenoberfläche. Diese Strecke ist zwischen Mitte der Sonnenscheibe und deren Rand um 0,47\% unterschiedlich. Außerdem variiert sie wegen der Elliptizität der Erdbahn zwischen $715 \mathrm{~km}$ und $735 \mathrm{~km}$.
} 


\section{Optischer Aufbau}

Der verwendete Beobachtungsaufbau (häufig als „Göttinger zweidimensionales SpektroPolarimeter“ oder kurz „2d-Spektro-Polarimeter" bezeichnet) befindet sich im Optiklabor II des Vakuum-Turm-Teleskops (VTT) (Soltau (1988) ) am Observatorio del Teide auf Teneriffa. Er entstand im Rahmen mehrerer Doktorarbeiten (Bendlin (1993), Volkmer (1995), Bendlin \& Volkmer (1995]) und wurde seitdem kontinuierlich erweitert und verbessert (Krieg (1999a). Ein wesentlicher Anteil der vorliegenden Arbeit bestand in der Implementierung eines zweiten Fabry-Perot-Interferometers (FPI), mit dessen Hilfe so kurze Belichtungszeiten für schmalbandige Filtergramme erreicht werden können, dass die Anwendung von Bildrekonstruktionsmethoden möglich ist. Weiterhin wurde eine zusätzliche CCD-Kamera installiert. Hiermit wurden Datensätze aufgenommen, mit deren Hilfe eine bestimmte Bildrekonstruktionsmethode (Phase Diversity, vgl. Kap. 5.1.6) angewendet werden sollte. Viele wesentliche Details des Beobachtungsaufbaus sind bereits in den o. g. Quellen beschrieben.

\subsection{Das Vakuum-Turm-Teleskop (VTT)}

Das Teleskop dient dazu, das von der Sonne kommende Licht zu sammeln und ein reelles Abbild eines Ausschnitts der Sonnenoberfläche zu erzeugen. Dieses reelle Bild im Primärfokus des Teleskops ist dann die Grundlage der weiteren Analyse des Sonnenlichts in dem nachfolgenden, eigentlichen Beobachtungsaufbau. Deshalb sollen hier nur kurz die für das Spektro-Polarimeter wichtigsten Eigenschaften wiedergegeben werden.

Das VTT ist ein klassisches Turmteleskop, d. h. das Teleskop ist starr und senkrecht in einem hohen Turm montiert. Das Licht des zu beobachtenden Objekts, hier also der Sonne, wird über ein Coelostatensystem, bestehend aus zwei plan geschliffenen Coelostatenspiegeln, senkrecht in den Turm eingespiegelt. Das Einstellen des Beobachtungsobjekts erfolgt manuell, die Nachführung der täglichen Erddrehung und die Feinnachführung (z. B. mechanischer oder langsamer atmosphärischer Abweichungen) geschehen dagegen vollautomatisch durch entsprechendes Drehen des jeweiligen Coelostatenspiegels.

Die Bauweise als Coelostat hat wesentliche Folgen: Zum einen findet trotz der Nachführung der Erddrehung keine Drehung des Bildfelds im Primärfokus statt. Dies ist von Vorteil, weil so nicht ständig das Bildfeld und/oder die Lage und Drehung der Detektoren nachgestellt werden müssen. 
Zum anderen hat dieser Aufbau aber den Nachteil, dass an den Coelostatenspiegeln nicht-senkrechte Reflexionen des Sonnenlichts auftreten, und die Reflexionswinkel sich zudem sowohl im Laufe des Jahres wie auch im Laufe eines Beobachtungstags verändern. Dadurch kommt es zu instrumenteller Polarisation und zum gegenseitigen Übersprechen der polarisierten Komponenten des Stokes-Vektors des beobachteten Lichts („Crosstalk“). Dies hat für polarimetrische Beobachtungen bestimmte Einschränkungen zur Folge, die in Kap. 3.3 kurz beleuchtet werden sollen.

Desweiteren wird bei flachen Einfallswinkeln des Sonnenlichts auf den ersten Coelostatenspiegel der Hauptspiegel des Teleskops nicht voll ausgeleuchtet. Das hat zur Folge, dass die Eintrittspupille des Teleskops nicht mehr kreisförmig ist, sondern ungefähr elliptisch wird. Damit ändert sich das theoretische Aufösungsvermögen des Teleskops für verschiedene Raumrichtungen in der Bildebene, die optische Transferfunktion (OTF) ist dann nicht mehr rotationssymmetrisch. Um diesen wichtigen Einfluss bei der späteren Bildrekonstruktion berücksichtigen zu können, wurde die Form der Eintrittspupille in regelmäßigen Abständen während kurzer Pausen zwischen aufeinanderfolgenden Beobachtungssätzen dokumentiert, indem sie direkt im Strahlengang des Spektro-Polarimeters fotografisch festgehalten wurde. Beispiele sind in Abb. 3.1 gezeigt. Neben der elliptischen
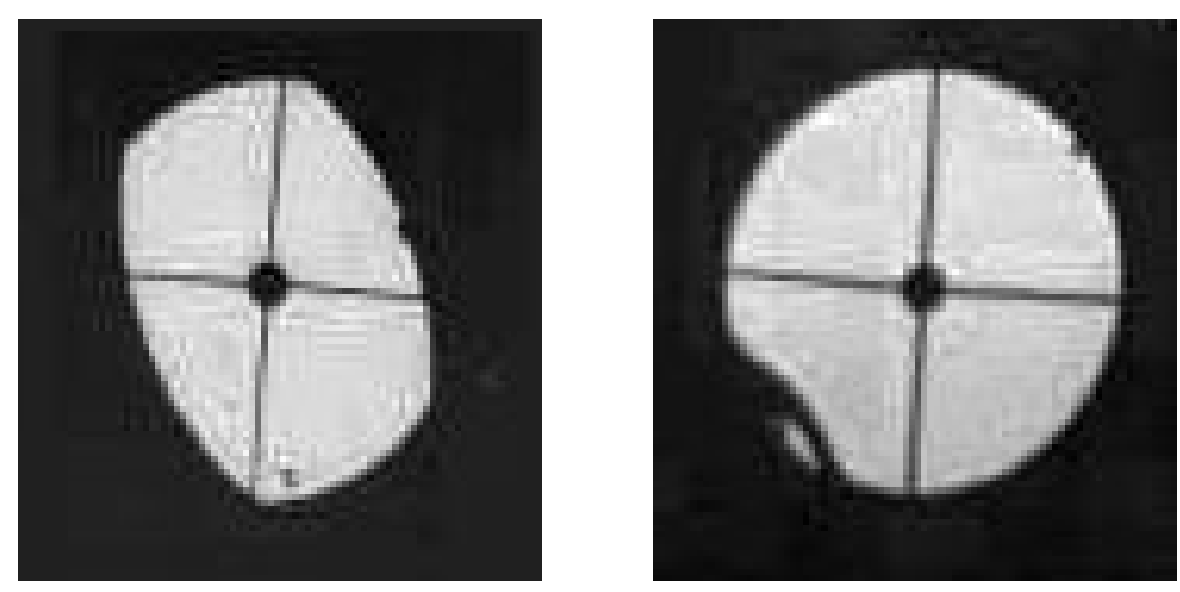

Abbildung 3.1: Fotografien des Pupillenbilds des VTTs bei verschiedenen Coelostatenstellungen.

Form sind in der Mitte des Pupillenbilds die Schatten eines kleinen Fangspiegels und seiner kreuzförmigen Halterung zu sehen. Der Fangspiegel wird für die Feinnachführung des Teleskops benötigt. Die Fotografien der Pupillenbilder wurden später digitalisiert und teilweise bei der Verarbeitung der Beobachtungsdaten berücksichtigt. In den meisten Fällen ist es indes gelungen, durch geschickte Wahl der Coelostaten-Stellung ein kreisrundes Pupillenbild zu erzeugen, das den Hauptspiegel voll ausleuchtet (in Abb. 3.1 rechts). Dadurch konnten Probleme bei der Bildrekonstruktion von vornherein ausgeschlossen werden. Der Einfluss des Fangspiegels und seines Haltekreuzes ist dabei vernachlässigbar klein, ebenso wie weitere geringe Abschattungen an den Rändern des Pupillenbilds. 
Das Teleskop selbst ist evakuiert, d. h. der größte Teil des Lichtwegs befindet sich im Vakuum. Dadurch wird erreicht, dass innerhalb des Teleskops keine die Abbildung störenden Seeingeinflüsse durch turbulente Luft auftreten. Das Licht tritt kurz hinter dem zweiten Coelostatenspiegel durch ein planparallel geschliffenes Eintrittsfenster aus optischem Glas in das Teleskop ein und ca. $2 \mathrm{~m}$ vor dem Primärfokus durch ein ähnliches (wenngleich kleineres) Austrittsfenster wieder aus.

Aufgrund der langen Brennweite des Hauptspiegels von $46 \mathrm{~m}$ ist das System als Schiefspiegler ausgelegt, der Strahlengang wird also durch einen Planspiegel, der gleichzeitig zum Fokussieren verwendet wird, gefaltet und tritt aus dem Austrittsfenster senkrecht nach unten aus. Über einen $45^{\circ}$-Spiegel wird das Licht dann in die Horizontale umgelenkt und steht der weiteren Verwendung in einem der optischen Labore zur Verfügung. Alternativ kann das Licht unter Auslassung des $45^{\circ}$-Spiegels auf einen Gitter-Spektrografen gelenkt werden.

Die wichtigsten technischen Daten des Teleskops können Tab. 3.1 entnommen werden.

\begin{tabular}{|c|c|}
\hline Durchmesser des Hauptspiegels $D$ & $70 \mathrm{~cm}$ \\
\hline Durchmesser der Coelostatenspiegel & $80 \mathrm{~cm}$ \\
\hline Brennweite $f$ & $46 \mathrm{~m}$ \\
\hline Öffnungsverhältnis $f / D$ & 65,7 \\
\hline Abbildungsmaßstab im Primärfokus & $4,59^{\prime \prime} / \mathrm{mm}$ \\
\hline Auflösungsvermögen bei $630,2 \mathrm{~nm}\left(1 / s_{\text {grenz }}\right)$ & $0,186^{\prime \prime}$ \\
\hline
\end{tabular}

Tabelle 3.1: Technische Daten des Vakuum-Turm-Teleskops (VTT) auf Teneriffa

\subsection{Das zweidimensionale Spektro-Polarimeter}

Im Folgenden soll der Aufbau des zweidimensionalen Spektro-Polarimeters in seiner aktuellsten Form beschrieben werden, wie es für die Aufnahme der vorliegenden Beobachtungsdaten verwendet wurde. Weitere Details können bei Bendlin (1993), Volkmer (1995) und Krieg (1999a) nachgeschlagen werden. Der gesamte Beobachtungsaufbau ist in einem optischen Labor des VTT fest installiert, nur wenige Teile werden zwischen den Beobachtungskampagnen abgebaut. Trotzdem muss die Anlage etwa einmal jährlich komplett justiert werden. Aufgrund der langen Lichtwege (der Hauptstrahlengang hat eine Länge von insgesamt etwa $15 \mathrm{~m}$ !) führen schon geringe mechanische Verschiebungen von Tischen und optischen Bänken zu merklichen Dejustierungen der Strahlengänge. Natürlich hinterlässt auch der tägliche Beobachtungsbetrieb Spuren wie Verschmutzungen und Fehljustierungen, die hin und wieder beseitigt werden müssen. Dazu sind selbstverständlich alle optischen Komponenten mit mechanischen Möglichkeiten zur Feinjustierung in allen Raumrichtungen ausgestattet. Ein schematisches Bild des Spektro-Polarimeters zeigt Abb. 3.2. 


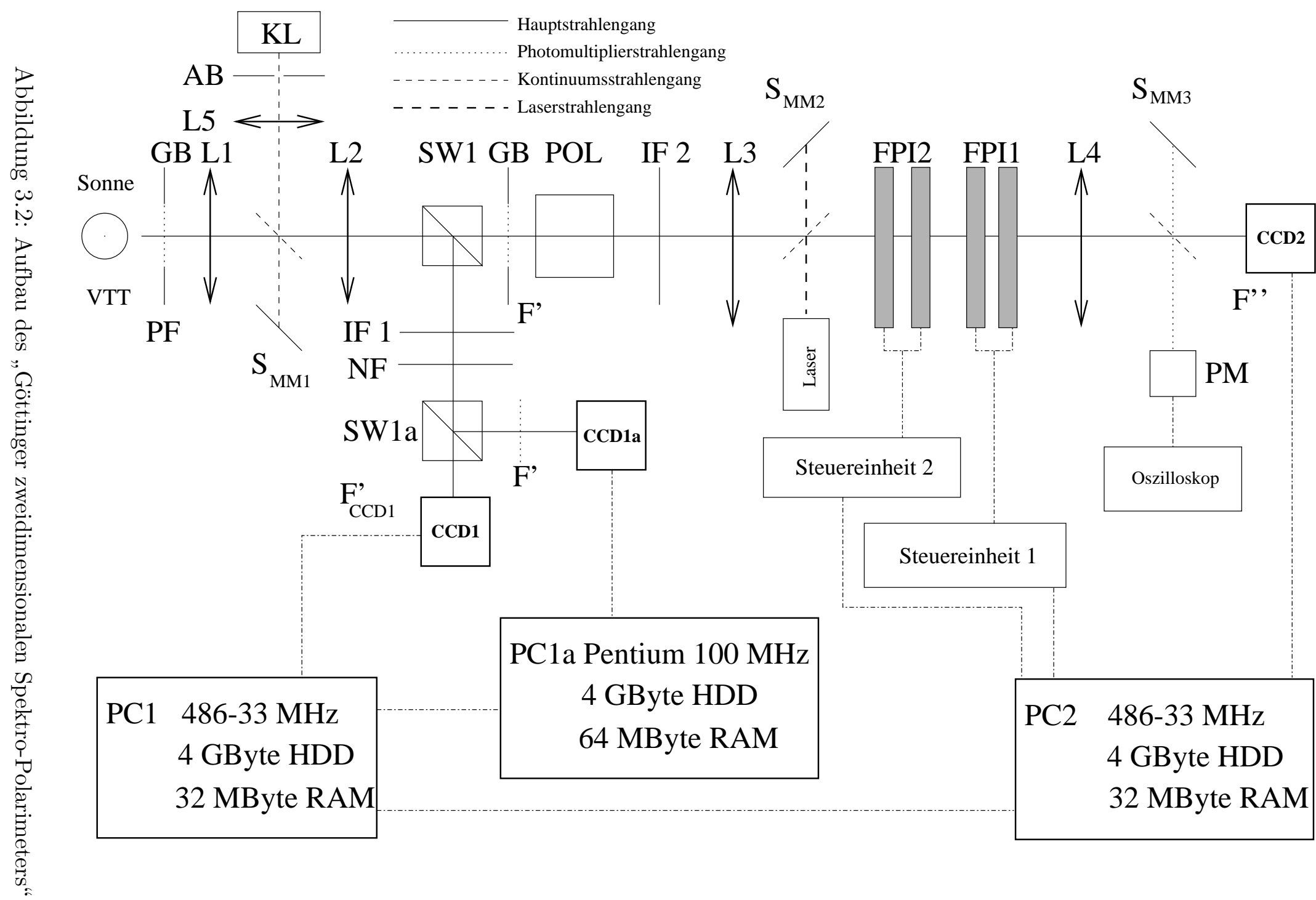




\subsubsection{Der Hauptstrahlengang}

Der wichtigste Teil der Anlage ist der Hauptstrahlengang, der beim Primärfokus des Teleskops mit Sonnenlicht beginnt, das sämtliche Wellenlängen enthält, die von den Spiegeln des Teleskops reflektiert und den Ein- und Austrittsfenstern des Vakuumtanks transmittiert werden. Er endet bei einem Abbild der Sonnenoberfläche auf einer CCDKamera (CCD 2) mit einer spektralen Bandbreite von einigen Pikometern.

Zunächst wird das Licht vom Primärfokus durch eine einfache Transferoptik aus dem Innenturm des VTT in das optische Labor OL 2 übertragen. Diese besteht aus zwei Achromaten L 1 und L 2 mit je $180 \mathrm{~cm}$ Brennweite, der Abbildungsmaßstab beträgt dabei 1:1. Die Linsen sind so aufgestellt, dass für den folgenden Bereich des Strahlengangs telezentrische Bedingungen herrschen. Dies wird erreicht, indem die zweite Transferlinse L 2 das von L 1 erzeugte Pupillenbild des VTTs ins Unendliche abbildet. Im größten Teil der Transferoptik verläuft das Lichtbündel innerhalb von geeignet montierten Kunststoffröhren. Dadurch wird vermieden, dass Turbulenzen innerhalb der Transferoptik die Bildqualität vermindern, quasi „internes Seeing“ verursachen. Bereits im Primärfokus wird mit Hilfe einer Irisblende der größte Teil des Sonnenbilds ausgeblendet, um möglichst wenig Streulicht im weiteren Verlauf der Beobachtungsanlage zu erzeugen.

Im Sekundärfokus wird mittels einer weiteren Blende das Bildfeld genau auf den später auf der CCD 2 sichtbaren Bildausschnitt reduziert. Das Licht passiert hinter dem Sekundärfokus das Stokes-V-Polarimeter, das bei Volkmer (1995) ausführlich beschrieben ist. Es wird hierbei in zwei parallele, seitlich gegeneinander versetzte Teilstrahlen aufgespalten. Die Hauptachsen der beiden Teilstrahlen liegen damit nicht mehr genau auf der optischen Achse des dahinter liegenden Aufbaus. Der Versatz beträgt aber nur wenige Millimeter. Da die nachfolgenden Abbildungen durch Linsen großer Brennweiten und Durchmesser erzeugt werden, ist in diesem Falle nicht mit einer Verschlechterung der Abbildungsqualität zu rechnen. Der Einfluss auf die Transmissionswellenlänge der nachfolgenden FPIs wird in Kap. 5.2.1 diskutiert.

Direkt hinter dem Polarimeter sorgt ein Interferenzfilter mit einer Halbwertsbreite von 0,3-2 nm für eine Vorauswahl der Wellenlänge. Bereits hier ist auf die Verwendung eines Filters mit hoher Transmission zu achten, damit möglichst kurze Belichtungszeiten erreicht werden können. Um den Einfluss auf die Bildqualität gering zu halten, werden Polarimeter und Interferenzfilter nah hinter dem Sekundärfokus montiert.

Im weiteren Verlauf wird das Licht durch eine weitere Transferoptik auf die CCD 2 abgebildet. Die beiden Linsen L 3 und L 4 haben Brennweiten von 225,4 cm und Durchmesser von $180 \mathrm{~mm}$, der Abbildungsmaßstab ist wieder 1:1. Zwischen L 3 und L 4, nahe des Pupillenbilds, wo das Lichtbündel am engsten ist, sind hintereinander zwei FPIs montiert, die in Kap. 3.2.2 näher beschrieben werden. Sie begrenzen die Wellenlänge des transmittierten Lichts auf wenige Pikometer, so dass die CCD 2 sehr schmalbandige Filtergramme aufnehmen kann. 
Aufgrund räumlicher Beschränkungen muss der Strahlengang hinter der Linse L 4 mit Hilfe eines Planspiegels gefaltet werden, der aber in Abb. 3.2 aus Platzgründen nicht gezeigt ist.

\subsubsection{Die Fabry-Perot-Interferometer (FPIs)}

\section{Grundlagen}

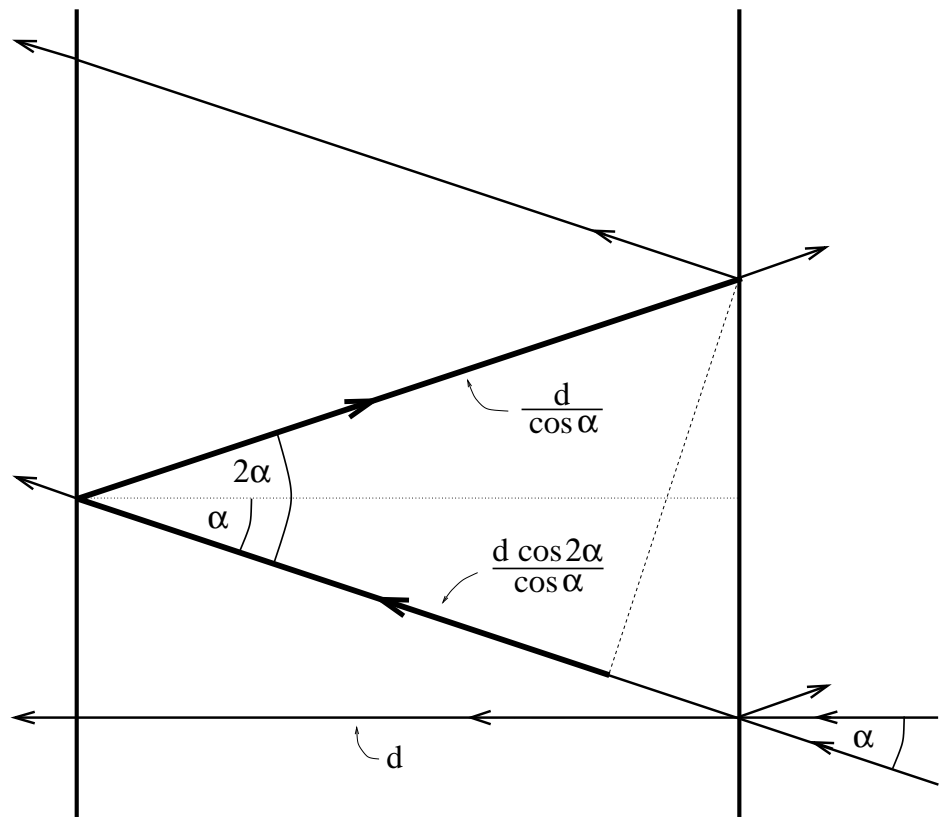

Abbildung 3.3: Reduzierter Plattenabstand für konstruktive Interferenz im FPI bei Einfall des Lichts unter einem Winkel $\alpha$ zur Senkrechten auf den Spiegelplatten.
Fabry-Perot-Interferometer (im Folgenden kurz FPIs genannt) bestehen im Wesentlichen aus zwei verspiegelten parallelen Glasplatten. Das Licht trifft ungefähr senkrecht auf die Platten auf und dringt in den Zwischenraum ein. Es wird zwischen den Platten mehrfach hin und her gespiegelt. Dabei tritt für solches Licht konstruktive Interferenz auf, das kohärent ist und für das der Wegunterschied, gegeben durch den Einfallswinkel $\alpha$ und den Plattenabstand $d$, ein ganzzahliges Vielfaches der Wellenlänge $\lambda$ ist. Abb. 3.3 zeigt, dass bei nicht senkrechtem Lichteinfall unter einem Winkel $\alpha$ zum Lot ein reduzierter Plattenabstand $d_{\mathrm{r}}$ angegeben werden kann. Dabei soll, wie beim senkrechten Lichteinfall, der Plattenabstand als halber Wegunterschied zwischen einem durchgehenden und einem einmal reflektierten Strahl definiert sein, und zwar so, dass die beiden parallelen Strahlen phasengleich sind:

$$
d_{\mathrm{r}}=\frac{1}{2}\left(\frac{d \cos 2 \alpha}{\cos \alpha}+\frac{d}{\cos \alpha}\right)=d \cos \alpha .
$$

Der Wegunterschied, der in Abb. 3.3 fett gezeichnet ist, ist also geringer als bei senkrechtem Durchgang des Lichts durch das FPI. Dies kommt einer Blauverschiebung der Transmissionswellenlänge $\lambda_{\mathrm{t}}$ gleich, es gilt:

$$
\lambda_{\mathrm{t}}(\alpha)=\lambda_{\mathrm{t}, \alpha=0^{\circ}} \cos \alpha .
$$

Dies zu berücksichtigen ist wichtig, da bei der Aufstellung der FPIs im parallelen Strahlengang der Einfallswinkel der Lichtstrahlen von der Position im Bildfeld abhängt (vgl. 
auch Kap. 5.2.1). Bei telezentrischer Aufstellung der FPIs dagegen unterscheiden sich die Einfallswinkel der Lichtstrahlen je nach ihrem Herkunftsort aus der Eintrittspupille des Instruments.

Im Prinzip gilt dieselbe Gleichung auch für Interferenzfilter, die ähnlich wie FPIs aufgebaut sind. Allerdings besteht bei ihnen der Zwischenraum zwischen den spiegelnden Flächen aus Glas oder ähnlichem Material, so dass der Brechungsindex dieses Materials noch berücksichtigt werden muss.

Für all das Licht, für das der zweifache reduzierte Plattenabstand nicht ein Vielfaches seiner Wellenlänge ist, findet bei der mehrfachen Reflexion zwischen den Spiegelplatten mehr oder weniger destruktive Interferenz statt. Aus genauen Rechnungen (Born \& Wolf (197[1]) ergibt sich eine Transmissionskurve, die das Verhältnis zwischen transmittiertem Licht $I_{t}$ und einfallendem Licht $I_{i}$ in Abhängigkeit von der Wellenlänge beschreibt. Diese heißt Airyfunktion:

$$
\frac{I_{t}}{I_{i}}(\lambda)=\frac{1}{1+F \sin ^{2} \frac{\delta(\lambda)}{2}} .
$$

Dabei sei $\delta(\lambda)$ die Phasendifferenz zwischen zwei interferierenden Strahlen:

$$
\delta(\lambda)=\frac{4 \pi}{\lambda} n d_{\mathrm{r}}
$$

mit dem Brechungsindex $n$ des Materials zwischen den Spiegelplatten. Der Faktor $F$ in Glg. 3.3 wird durch die Reflektivität der Spiegelplatten bestimmt. Dadurch ergeben sich Transmissionskurven, wie sie in Abb. 3.4 gezeigt sind. In bestimmten Abständen befinden sich bei einem gegebenen Plattenabstand $d_{\mathrm{r}}$ Transmissionsmaxima für die Wellenlängen $\lambda_{\mathrm{t}, m}\left(m \in \mathbb{N}^{+}\right)$, für die der zweifache Plattenabstand ein Vielfaches der Wellenlänge bei (mit abnehmender Wellenlänge) zunehmender Ordnung $m$ ist. Dieser Abstand wird „freier Spektralbereich“ (englisch: „free spectral range“, FSR) genannt, und es gilt:

$$
\mathrm{FSR}=\frac{\lambda^{2}}{2 d_{\mathrm{r}}}
$$

Ebenfalls von der Reflektivität der Spiegelplatten hängt das Verhältnis zwischen dem FSR und der Halbwertsbreite eines einzelnen Durchlassbereichs $\Delta \lambda$ ab, es heißt Finesse $\mathcal{F}$ :

$$
\mathcal{F}=\frac{\text { FSR }}{\Delta \lambda}
$$

Von ihr hängt das spektrale Aufösungsvermögen des Beobachtungsaufbaus ab. Da die real messbare Finesse aber nicht nur durch die Reflektivität der Spiegelplatten bestimmt wird, sondern z. B. auch durch die Unebenheit, mit der die Spiegel geschliffen sind, wird i. A. eine effektive Finesse $\mathcal{F}_{\text {eff }}$ angegeben, die diese Einflüsse berücksichtigt. Auch Fehljustierungen der Plattenparallelität gehen im Prinzip als Unebenheiten der Spiegel in $\mathcal{F}_{\text {eff }}$ ein (Atherton et al. (1981) $)$. Ein weiterer Einfluss auf $\mathcal{F}_{\text {eff kann dadurch gegeben }}$ sein, dass das Lichtbündel nicht kollimiert, sondern konvergent ist, z. B. wenn das FPI 
im telezentrischen Strahlengang aufgestellt ist. Dann werden die Durchlassbereiche um so breiter, je kleiner das Öffnungsverhältnis $f / D$ des Bündels ist (Rust (1991)). $\mathcal{F}_{\text {eff }}$ lässt sich unter entsprechender Berücksichtigung aller Einflüsse berechnen, Details hierzu finden sich bei Atherton et al. (1981), Vaughan (1989), und Bendlin (1993).

\section{Ein Doppel-FPI}

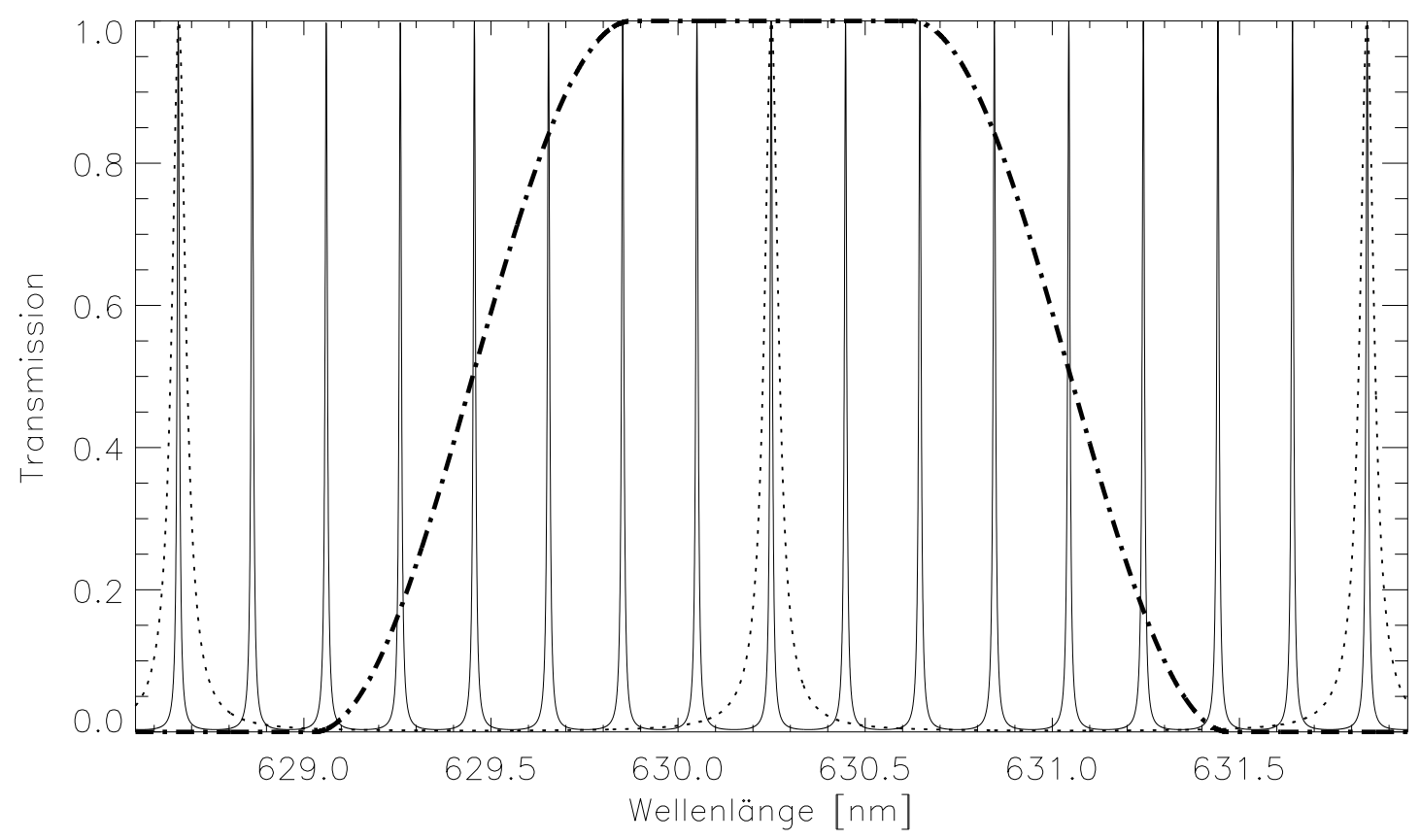

Abbildung 3.4: Transmissionskurven der FPIs und des Interferenzfilters. Durchgezogen die Airyfunktion von FPI 2 (mit $\mathcal{F}_{\text {eff }} \approx 30$ ), gepunktet die Airyfunktion von FPI 1 (mit $\mathcal{F}_{\text {eff }} \approx 35$ ) und strichpunktiert eine willkürlich angenommene Interferenzfilterkurve (1,6 nm Halbwertsbreite). Die Werte gelten für einen Plattenabstand $d_{2}$ von FPI 2 von $1 \mathrm{~mm}$.

Das Funktionsprinzip des Spektrometers ist, dass die CCD 2 nur das Licht aus einem bestimmten Durchlassbereich der Airyfunktion von FPI2 (durchgezogene Kurve in Abb. 3.4) erhalten soll, so dass ein schmalbandiges Filtergramm entsteht. Dafür muss aber das Licht aller anderen Durchlassbereiche ausgeblendet werden. Dies geschieht durch das nachgeschaltete FPI 1 mit geringerem Plattenabstand $d_{1}$. Dieses hat daher (Glg. 3.5) einen größeren FSR, so dass alle benachbarten Durchlassbereiche von FPI 2 herausgefiltert werden (gepunktete Kurve). Um das Licht aus den weiteren Durchlassbereichen von FPI 1 ebenfalls auszublenden, wird ein Interferenzfilter verwendet, das auf

\footnotetext{
${ }^{1}$ Die Reihenfolge, in der die beiden FPIs im Strahlengang montiert werden, ist dabei physikalisch irrelevant und hat lediglich mechanische Gründe.
} 
dem gleichen Prinzip wie ein FPI beruht, aber nur einen einzigen Durchlassbereich hat (strichpunktierte Kurve).

Die Durchlasskurve des schmalbandigen FPI 2 bestimmt das spektrale Auflösungsvermögen der Anlage, das breitbandigere FPI 1 und das Interferenzfilter dienen lediglich zum Heraussortieren des gewünschten Durchlassbereichs von FPI 2, also dem Filtern aller unerwünschter Ordnungen.

Diese Aufgabe wurde früher von einem durchstimmbaren Lyot-Filter und einem Interferenzfilter erledigt (Bendlin (1993)] . Das Lyot-Filter hatte aber den Nachteil einer sehr geringen Transmission von wenigen Prozent, was zu unerwünscht langen Belichtungszeiten führte. Deshalb wurde es im Rahmen dieser Arbeit (zusammen mit der Arbeit von Krieg (1999a) gegen das besagte FPI 1 ausgetauscht. Die technischen Daten der FPIs wurden dabei so aufeinander abgestimmt, dass eine hohe Transmission bei möglichst wenig Falschlicht (aus anderen Ordnungen von FPI2) erreicht werden konnte. Ein weiterer Kompromiss musste zwischen spektraler Auflösung und Lichtstärke gefunden werden. Es ist klar, dass, je schmaler der Durchlassbereich des FPI 2 ist, desto weniger Photonen insgesamt in dem Filtergramm an CCD 2 registriert werden. Der Plattenabstand $d_{2}$ von FPI 2 wurde deshalb so gewählt, dass in den Filtergrammen bei Belichtungszeiten von 20 - 40 ms hinreichend große Intensitäten gemessen werden können, die ein für die Anwendung von Bildrekonstruktionsmethoden hinreichendes Signal-zu-Rausch-Verhältnis (englisch „signal-to-noise-ratio“, SNR) aufweisen.

Ein früher eingesetzter Bildverstärker vor CCD 2 wurde hier nicht verwendet, weil er zwar die absolut gemessenen Intensitäten erhöht, aufgrund seiner geringen Quantenausbeute von nur ca. $10 \%$ aber das SNR gegenüber der CCD-Kamera mit einer Quantenausbeute von ca. $50 \%$ verringert.

Andere Beobachtungsaufbauten, bei denen Einfach-, Doppel- oder Dreifach-FPIs verwendet oder geplant werden, sind u. a. bei Mack et al. (1963), Atherton et al. (1981), Rust (1991), Kentischer et al. (1998) und v. d. Lühe \& Kentischer (2000) beschrieben.

\section{Technische und optische Daten}

Bei den beiden verwendeten FPIs (vgl. Abb. 3.2) werden bei optimal parallel justierten Platten und senkrechtem Lichteinfall über weite Wellenlängenbereiche effektive Finessen $\mathcal{F}_{\text {eff }}$ von 40 - 45 bei FPI 1 und 35-40 bei FPI2 erreicht. Typische Reflektivitäten der Spiegelplatten sind 94-97\%, die Plattenunebenheiten liegen in der Größenordnung $\lambda / 150-\lambda / 200$.

Bei den in Abb. 3.4 angenommenen Werten von $\lambda=630,25 \mathrm{~nm}$ und $d_{2}=1 \mathrm{~mm}$ ist die Intensität in den Transmissionsmaxima, die dem gewünschten Durchlassbereich benachbart sind („Falschlicht“), bei der Gesamtfilterkurve (also dem Produkt der drei gezeigten Transmissionskurven) ca. 1,5\% der einfallenden Intensität.

All diese Angaben gelten für den idealisierten Fall, dass bei den Reflexionen und Trans- 


\begin{tabular}{|l||c|c|}
\hline \multicolumn{1}{|c||}{} & FPI 1 & FPI2 \\
\hline \hline Hersteller & Queensgate Instruments Ltd. & Burleigh Instruments Inc. \\
\hline Typ & ET 50 FS & RC-150 \\
\hline Steuergerät & CS 100 Controller & RC-43/44 Ramp Generator \\
\hline Freie Öffnung & $50 \mathrm{~mm}$ & $35 \mathrm{~mm}$ \\
\hline Ebenheit der Spiegel & $\lambda / 150$ & $\lambda / 200$ \\
\hline Reflektivität & $95-97 \%$ & $94 \%$ \\
\hline Max. Transm. $(632,8 \mathrm{~nm})$ & ca. $80 \%$ & $50-60 \%$ \\
\hline Effektive Finesse $\mathcal{F}_{\text {eff }}$ & $40-45$ & $35-40$ \\
\hline Plattenabstand $d$ & $125 \mu \mathrm{m}$ & $1-2 \mathrm{~mm}$ \\
\hline FSR (bei $630,25 \mathrm{~nm})$ & $1,59 \mathrm{~nm}$ & $5,197 \mathrm{~nm}\left(d_{2}=1 \mathrm{~mm}\right)$ \\
\hline Halbwertsbreite $\Delta \lambda$ & $40-35 \mathrm{pm}$ & ca. $120000\left(d_{2}=1 \mathrm{~mm}\right)$ \\
\hline Auflösungsverm. $\lambda / \Delta \lambda$ & ca. 17000 & $\mathrm{~mm})$ \\
\hline
\end{tabular}

Tabelle 3.2: Technische und optische Daten der Fabry-Perot Interferometer (FPIs)

missionen keinerlei Absorption auftritt. Diese ist in der Praxis natürlich immer vorhanden und verringert die maximale Transmission. Einfache Messungen mit Laserlicht haben für das FPI 1 eine Maximaltransmission von ca. $80 \%$ ergeben, für das FPI 2 einen Wert von 50-60\%. Dabei bleiben jedoch die effektiven Finessen unverändert.

Die technischen und optischen Daten der beiden FPIs sind in Tab. 3.2 aufgeführt. Beide FPIs werden über elektronische Steuergeräte betrieben, dabei werden Plattenabstand und Plattenparallelität über hochspannungsgeregelte piezokeramische Elemente gesteuert. Während das neuere FPI 1 über eine sehr gut funktionierende automatische Stabilitätskontrolle verfügt, muss beim älteren FPI 2 die Plattenparallelität und mithin die effektive Finesse in regelmäßigen Abständen, auch während der Beobachtungen, nachgeregelt werden. Diese Einstellung muss, je nach Umgebungsbedingungen (Temperatur, Luftfeuchtigkeit, Erschütterungen, etc.) und „Tagesform“ des FPIs im Abstand von zehn Minuten bis zu einigen Stunden erfolgen. Beim FPI 2 ist außerdem der Plattenabstand auf mechanischem Wege grob zwischen null und mehreren Millimetern verstellbar. Der sinnvolle Einsatzbereich im vorliegenden Aufbau liegt zwischen einigen hundert Mikrometern und $2 \mathrm{~mm}$.

Die Steuergeräte sind wiederum mit einem Personal Computer verbunden (PC2 in Abb. 3.2), so dass die Steuerung während der Aufnahmen automatisiert in den Beobachtungsablauf eingebunden werden kann. Zur Ansteuerung des neueren FPI 1 mussten in Zusammenarbeit mit der elektronischen Werkstatt umfangreiche Hardware-Komponenten entwickelt und eingebaut werden, sowie die Steuerungs-Software des Spektrometers an das neue Gerät angepasst werden. 


\section{Blauverschiebung}

Die Aufstellung der FPIs im parallelen Strahlengang hat zur Folge, dass Licht von Bildpunkten, die nicht in der Mitte des Bildfelds liegen, leicht geneigt gegen die optische Achse des Aufbaus durch die FPIs treten. Das führt zu den weiter oben bereits erwähnten Blauverschiebungen der Transmissionswellenlänge über das Bildfeld.

Wird der Aufbau ohne Polarimeter verwendet, so ergibt sich mit einem Bildfeld von $29,5^{\prime \prime} \times 39,5^{\prime \prime}$ in den Bildecken ein maximaler Winkel von $0,14^{\circ}$ gegen die optische Achse. Daraus resultiert bei einer Wellenlänge von 630,25 nm nach Glg. 3.2 eine Blauverschiebung der Transmissionswellenlänge von 1,9 pm gegenüber dem Mittelpunkt des Bildfelds, vorausgesetzt, dass das FPI genau senkrecht zur optischen Achse des einfallenden Lichts montiert ist.

Wird zusätzlich das Polarimeter verwendet, halbiert sich das Bildfeld und folglich treten noch geringere Winkel und Blauverschiebungen auf. Auf die winzigen Abweichungen, die durch den seitlichen Versatz der polarisierten Teilstrahlen entstehen, wird in Kap. 5.2.1 eingegangen.

\section{Reflexe}

Zwischen den beiden hintereinander montierten FPIs kommt es zu Reflexen von Licht, das teilweise mehrfach zwischen den FPIs hin und her reflektiert wird, dann aber letztlich doch wenigstens z. T. auf der CCD 2 landet, wo es zu unerwünschtem Falschlicht und Geisterbildern führt. Gerade Licht aus den Flanken der FPI-Durchlassbereiche kann zu einem ganz erheblichen Anteil nach Mehrfachreflexionen den Detektor erreichen. Um dies $\mathrm{zu}$ vermeiden, wurde das breitbandigere FPI 1 soweit gekippt, dass die Reflexe gerade eben neben dem CCD-Chip der Ka-

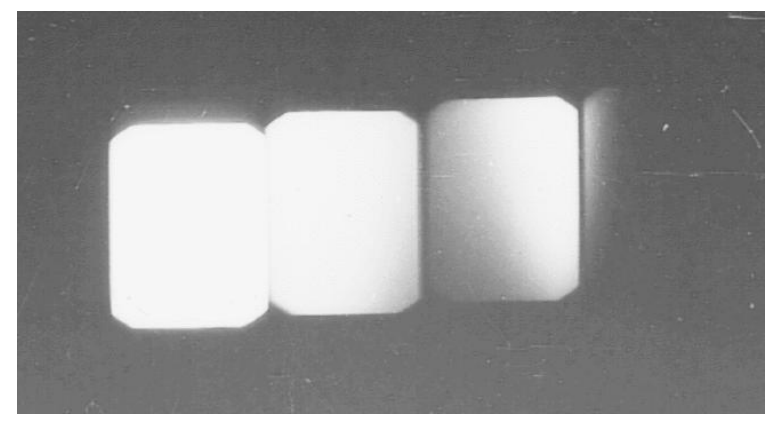

Abbildung 3.5: Reflexe zwischen den FPIs, aufgenommen in schmalbandigem Licht (mit IF 2) ohne Polarimeter. Links das Hauptlichtbündel, rechts daneben Reflexe steigender Ordnung. mera zu liegen kamen. In Abb. 3.5 ist eine Fotografie des Fokusbereichs der CCD 2 zu sehen. Wiederum gelten die folgenden Abbildungen und Überlegungen für den Einsatz der Anlage ohne Polarimeter. Sie lassen sich aber leicht auf die Anwendung mit Polarimeter übertragen. Man erkennt rechts neben dem hellen Hauptstrahlengang mehrere seitlich versetzte Reflexe. Der erste stammt von einer Einfachreflexion zwischen den FPIs, der nächste von einer Zweifachreflexion, usw. Weitere Reflexionen finden auf den unverspiegelten, aber leicht angeschrägten Rückseiten der FPI-Spiegelplatten statt, weshalb in einer länger belichteten Fotografie (Abb. 3.6) 


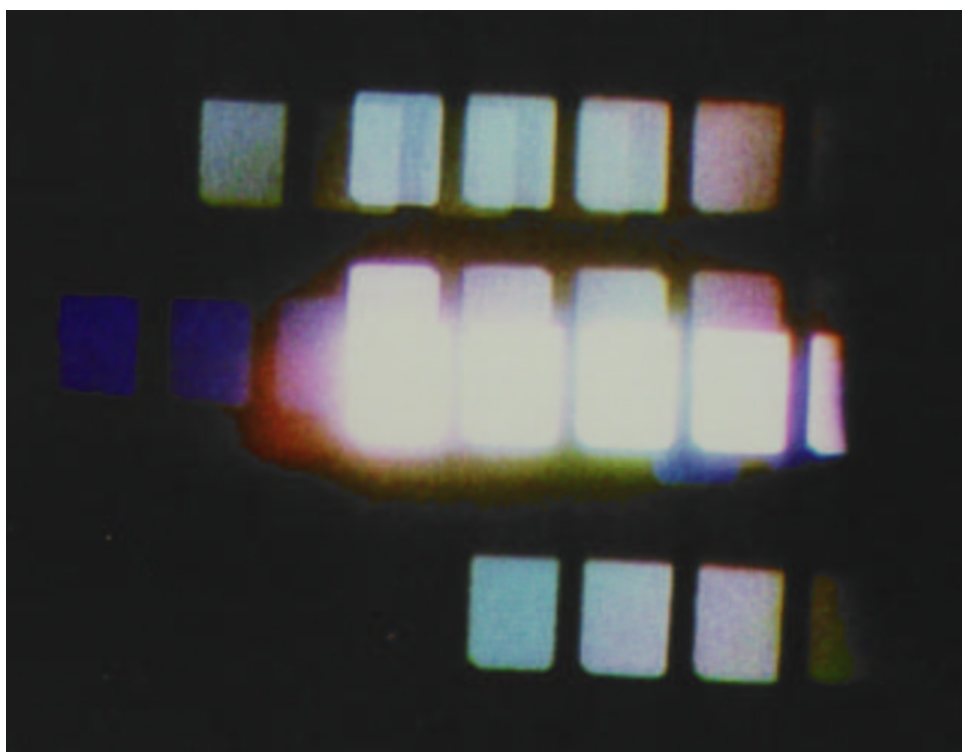

Abbildung 3.6: Reflexe zwischen den FPIs, aufgenommen in breitbandigem Licht (ohne IF 2) ohne Polarimeter, lang belichtet. Knapp links der Bildmitte das Hauptlichtbündel, rechts daneben, darüber und darunter Reflexe. weitere, nach oben und unten versetzte Reihen von Reflexen sichtbar sind. Ebenso überlappt auch bei der vorliegenden Kippung von FPI 1 eine Reihe sehr schwacher Reflexe die Hauptreflexreihe und damit auch das Hauptlichtbündel, jedoch sind diese Reflexe so schwach, dass sie nicht berücksichtigt werden müssen.

Damit der Winkel, um den das FPI gegen die optische Achse des Beobachtungsaufbaus gekippt werden muss, so gering wie möglich gehalten werden kann, muss darauf geachtet werden, dass mit Hilfe der

Rechteckblende im Sekundärfokus (Abb. 3.2) der Bildausschnitt auf die minimale Größe verkleinert wird, gerade ohne das Bildfeld auf CCD 2 zu beschränken. So wird mit einer Kippung um $0,08^{\circ}$ dafür gesorgt, dass der erste Reflex gerade eben aus dem Bildfeld von CCD 2 herauswandert. Dieser Wert ist gerade der Winkel zwischen den Lichtstrahlen von der Mitte der langen Bildfeldkante und der optischen Achse am Ort der FPIs im parallelen Strahlengang.

Nach Glg. 3.2 führt die zusätzliche Kippung von FPI 1 zu einer Blauverschiebung der Transmissionswellenlänge von 2,7 pm zwischen dem linken und rechten Bildrand. In Verbindung mit der Wellenlängenvariation von FPI2, das exakt senkrecht zur optischen Achse montiert ist, ergibt sich bei optimaler Einstellung der beiden Durchlassbereiche aufeinander ein linearer Verlauf der Wellenlängendifferenz zwischen den Durchlassbereichen quer über das Bildfeld. Die Maximalwerte der Wellenlängendifferenz betragen dann $\pm 1,35$ pm (Abb. 3.7). Diese Differenzen sind in Anbetracht der Halbwertsbreiten der FPIs (vgl. Tab. 3.2) zu vernachlässigen, sie führen nicht zu einem merklichen Abfall der Gesamttransmission des Systems. Entlang der langen Kanten des Bildfelds sind die Wellenlängendifferenzen zwischen den Durchlassbereichen der beiden FPIs konstant.

Weiterhin wird durch das Kippen des FPI 1 seine effektive Finesse geringfügig verschlechtert, für die Beobachtungen sind Werte von $\mathcal{F}_{\text {eff }} \approx 35$ realistisch. 


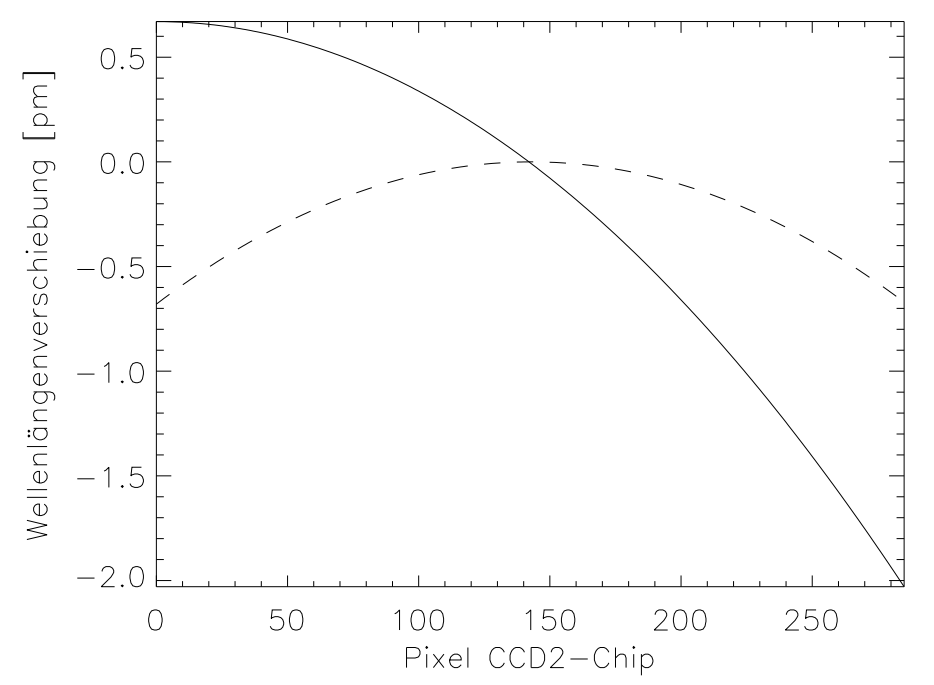

Abbildung 3.7: Wellenlängenverlauf der Transmissionsmaxima von FPI 1 (durchgezogen) und FPI 2 (gestrichelt) quer über die schmale Seite des Bildfelds durch dessen Mittelpunkt. FPI 1 ist dabei so gekippt, dass die Reflexe genau seitlich neben der CCD 2 zu liegen kommen (Abb. 3.5). Angegeben ist die Wellenlängendifferenz zur maximalen Transmissionswellenlänge von FPI 2 genau in der Bildmitte.

\section{Parallelfahren der FPIs}

Während der Aufnahmen sollen die Durchlassbereiche der FPIs computergesteuert verändert werden, um nach und nach eine Spektrallinie mit einzelnen Filtergrammen abzutasten. Dazu ist es unbedingt notwendig, dass die beiden FPIs mit exakt gleichen Geschwindigkeiten parallel in Wellenlängenrichtung fahren. Sonst würden nach und nach die Durchlassbereiche der FPIs nicht mehr übereinstimmen und die Transmission des Gesamtsystems würde sinken.

Durch einen Regler in der elektronischen, analogen Datenübertragung zwischen dem PC 2 und dem Steuergerät des FPI 1 konnte die Fahrgeschwindigkeit des FPI 1 in gewissem Maße geregelt und an die Geschwindigkeit des FPI 2 angepasst werden. Getestet wird der Parallellauf der beiden FPIs, indem man den Durchlassbereich von FPI 2 in eine der Flanken vom Durchlassbereich von FPI 1 stellt und dann in einem Bereich des Sonnenspektrums, der möglichst keine Spektrallinien enthält, über einen möglichst weiten Spektralbereich fährt. Wenn dann die Intensität, also die Gesamttransmission des Systems, konstant bleibt, sind die Fahrgeschwindigkeiten der beiden FPIs in Wellenlängenrichtung identisch. Vorausgesetzt werden muss natürlich, dass das Sonnenspektrum in diesem Bereich flach ist und auch das Interferenzfilter (IF 2) eine flache Transmissionskurve hat. 


\subsubsection{Der Breitbandkanal}

Kurz vor dem Sekundärfokus (F' in Abb. 3.2) ist ein Strahlteilerwürfel (SW 1) montiert. Dieser lenkt 5\% des Lichts aus dem Hauptstrahlengang heraus. Dieses Licht gelangt durch ein Interferenzfilter (IF) und ein Neutralfilter (NF) auf eine weitere CCD-Kamera (CCD 1). Direkt vor CCD 1 jedoch befindet sich ein weiterer Strahlteilerwürfel (SW 1a), der das Licht zu je $50 \%$ aufteilt und die eine Hälfte auf eine dritte CCD-Kamera (CCD 1a) lenkt. Die grundsätzliche Idee des Aufbaus ist es, in einem Hauptstrahlengang schmalbandige Filtergramme aufzunehmen (Kap. 3.2.1) und exakt gleichzeitig in einem optisch möglichst gleichartigen Nebenstrahlengang, der an geeigneter Stelle vom Hauptstrahlengang abgezweigt wird, breitbandige Aufnahmen desselben Ausschnitts der Sonnenoberfläche aufzunehmen. Die breitbandigen Aufnahmen haben gegenüber den Filtergrammen den Vorteil, ein wesentlich höheres Signal-zu-Rausch-Verhältnis (SNR) aufzuweisen, die Strukturen sind sehr viel deutlicher sichtbar und somit kontrastreicher. Deshalb lassen sich wesentliche Informationen, die sowohl in den Filtergrammen wie auch in den breitbandigen Aufnahmen gleichermaßen enthalten sind, leichter aus den letzteren ermitteln. Zu diesen Informationen gehören im einfachsten Fall die Bildverschiebungen (,image motion“), jedoch auch die weitergehenden Informationen über die atmosphärischen Abbildungseigenschaften zur Zeit der Aufnahmen, also das Seeing. Wenn es also gelingt, aus den breitbandigen Aufnahmen durch Bildrekonstruktionsmethoden Informationen über die momentane optische Transferfunktion (OTF) zu berechnen, so können diese verwendet werden, um die schmalbandigen Filtergramme aus dem Hauptstrahlengang zu entfalten und auch aus diesen die störenden atmosphärischen (und evtl. instrumentellen) Einflüsse zu eliminieren.[]

Damit das gelingen kann, müssen die beiden Strahlengänge weitgehend identisch sein, bis auf die spektrale Bandbreite, deren Unterschied ja gerade das höhere SNR im Breitbandkanal ausmachen soll. Die Zentralwellenlänge der beiden Aufnahmen darf sich jedoch nicht zu stark unterscheiden, da die atmosphärischen Einflüsse in gewissem Maße von der Wellenlänge abhängen. Deshalb werden als Interferenzfilter (IF 1) immer solche Filter ausgewählt, deren Zentraldurchlasswellenlänge jeweils mit der Wellenlänge der schmalbandigen Filtergramme übereinstimmen, und eine Bandbreite von 5-10 nm haben. So ist immer gewährleistet, dass in diesem Kanal genügend Photonen für ein hohes SNR zur Verfügung stehen. In der Praxis muss die Intensität sogar immer noch durch ein zusätzliches Graufilter (NF) abgeschwächt werden.

Weiterhin müssen die Kameras exakt simultan belichtet werden, da sich das atmosphärische Seeing innerhalb sehr kurzer Zeit (wenige Millisekunden) ändern kann. Die beiden CCD-Kameras CCD 1 und CCD 2 sind deshalb über ihre Steuerrechner PC 1 und PC 2

\footnotetext{
${ }^{2}$ Das heißt nicht automatisch, dass die Bilder in den beiden Kanälen ein ähnliches Aussehen haben müssten: Ein schmalbandiges Filtergramm aus dem Kern einer starken Spektrallinie kann völlig anders aussehen als ein breitbandiges Bild bei derselben Zentralwellenlänge. Wichtig ist, dass die die Abbildungsqualität verringernden Einflüsse in beiden Bildern identisch sind.
} 
so verbunden, dass sie exakt gleichzeitig die Belichtung auslösen. Die Triggerimpulse für CCD 1 werden durch Auftrennen des Steuerkabels ebenfalls für die Triggerung von CCD 1a verwendet, so dass auch für diese Kamera eine simultane Belichtung der Aufnahmen gewährleistet ist.

Aufgrund der optischen Anordnung ist weiterhin gewährleistet, dass alle drei Kameras denselben Abbildungsmaßstab haben. Dass sie denselben Bildausschnitt aufnehmen, muss durch eine genaue Justierung der optischen Komponenten sichergestellt werden. An den Strahlteilerwürfeln werden die Bilder jeweils für die eine Aufspaltungsrichtung reflektiert, für die CCD 2 findet außerdem noch eine weitere Abbildung durch L3 und L4 und eine Spiegelung statt. Deshalb müssen später in der Datenauswertung noch die Bilder durch geeignete Spiegelungen und Drehungen zur Deckung gebracht werden (vgl. Kap. 5.1.1).

\section{CCD 1a - die „Phase-Diversity-Kamera“}

Während CCD 1 im Fokus steht, und damit, von der spektralen Bandbreite abgesehen, dasselbe Bild aufnehmen sollte wie CCD 2, ist die CCD 1a um einen bestimmten, einstellbaren Wert aus der Fokalebene heraus verschoben. Es sollen auf diese Weise mit der Kamera Informationen über das der Fokalebene umliegende Volumen erhalten werden. Aus diesen Informationen kann die Form der in das Teleskop einlaufenden Wellenfronten bestimmt werden. Die Methode heißt deshalb „Fokus-Volumen-Methode“ oder auch „Phase Diversity“. Sie soll in einem späteren Kapitel (5.1.6) genauer beschrieben werden. Die Kamera CCD 1a, die für die Anwendung dieser Methode benötigt wird, musste im Rahmen der vorliegenden Arbeit in das bestehende Spektro-Polarimeter integriert werden. Dazu waren verschiedene Hard- und Softwareanpassungen notwendig. Auch die mechanische Installation der optischen Komponenten musste geplant und durchgeführt werden. Die Kamera wurde auf einem in alle Richtungen verstellbaren Kreuzschlitten montiert.

Bei der Justierung der Beobachtungsanlage wird die Kamera zunächst genau wie CCD 1 exakt in den Fokus gebracht, dazu wird ein im Primärfokus aufgestelltes Testobjekt (Siemens-Stern) zu Hilfe genommen. Von dieser Stellung ausgehend wird dann die Kamera um einen definierten Betrag aus dem Fokus herausgefahren. Die meisten Beobachtungen wurden mit einer Fokusdifferenz aufgenommen, die einem Wellenfrontfehler von $1 \lambda$ entspricht, d. h., dass am Ort der Beobachtung (also auf dem CCD-Chip) für Lichtstrahlen aus der Mitte der Eintrittspupille eine Phasendifferenz $\Delta \phi$ von $2 \pi$ gegenüber Strahlen vom Rand der Eintrittspupille besteht. Anders ausgedrückt, es verlängert sich beim Defokussieren der Lichtweg für einen Strahl vom Rand der Eintrittspupille um genau eine Wellenlänge weniger, als für einen Strahl durch die Mitte. Abb. 3.8 zeigt die Verhältnisse: Bei Verlängerung der Beobachtungsdistanz um $\Delta z$ verlängert sich der Lichtweg für einen Mittelpunktsstrahl mehr als für einen Randstrahl. 


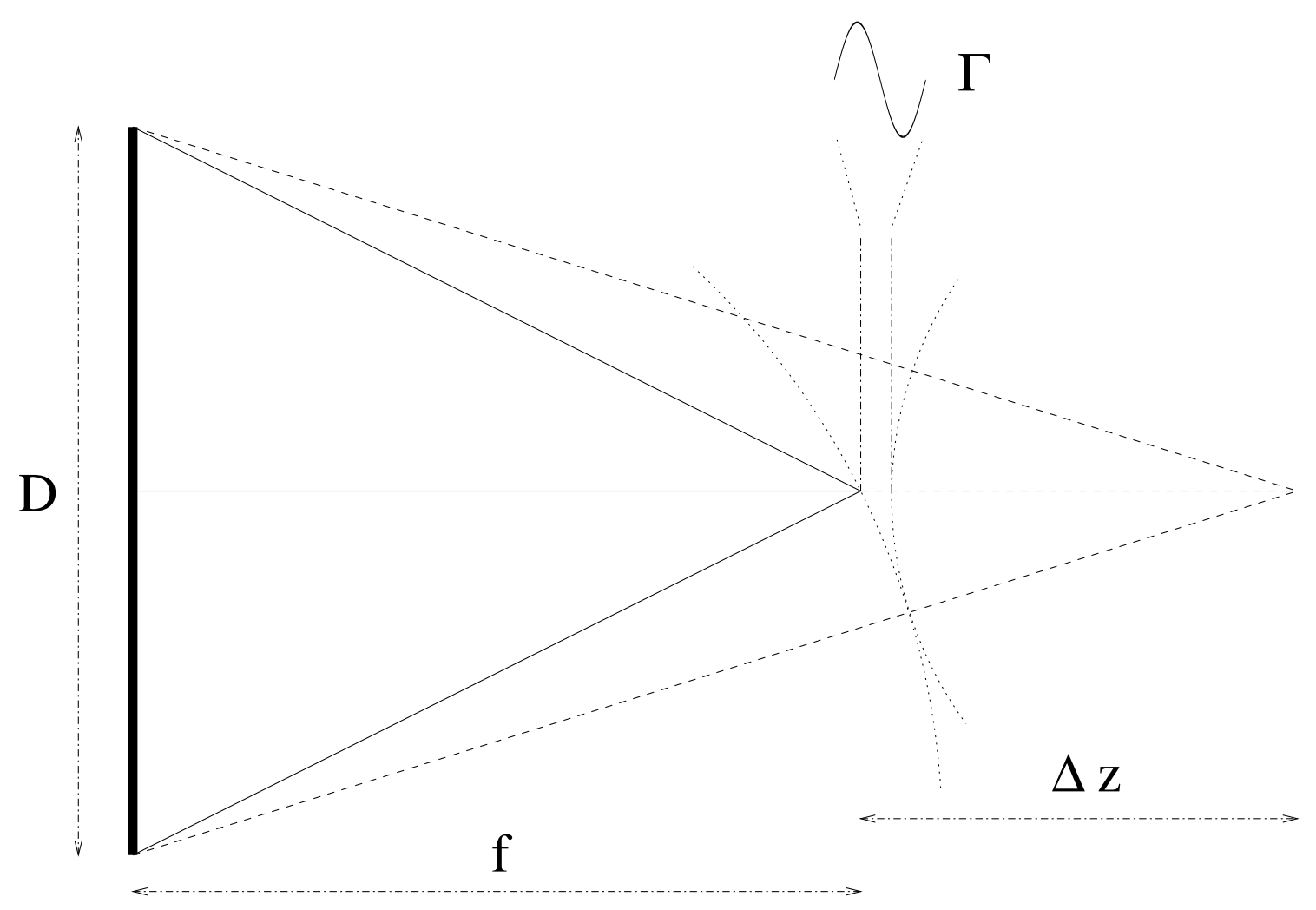

Abbildung 3.8: Wellenlängenaberration bei Vergrößerung der Distanz zwischen Detektor und Brennebene

Die Fokusdifferenz, die nötig ist, um eine bestimmte Wellenfrontaberration $\Gamma=\Delta \phi / 2 \pi$ zu erzeugen, errechnet sich wie folgt:

$$
\Delta z=8 \lambda\left(\frac{f}{D}\right)^{2} \cdot \Gamma .
$$

Dabei ist $(f / D)$ das Öffnungsverhältnis des Teleskops, hier also 65,7. Damit ergibt sich für $\Gamma=1$ und für eine Lichtwellenlänge $\lambda=630,25 \mathrm{~nm}$ eine Fokusdifferenz von $\Delta z=$ 21, $8 \mathrm{~mm}$. Da die Informationen vor und hinter der Fokalebene redundant sind, ist es im Übrigen egal, ob die Kamera um diesen Betrag intra- oder extrafokal postiert wird.

Nach der Einstellung der Fokusdifferenz muss noch der genaue Bildausschnitt justiert werden. Dazu wird wie üblich ein im Primärfokus plaziertes Gittermuster verwendet. Damit dieses noch trotz der Defokussierung hinreichend gut auf der CCD 1a zu erkennen ist, muss der Strahlengang für diese Justierung abgeblendet werden, indem eine Irisblende von ca. $1 \mathrm{~cm}$ Durchmesser im Pupillenbild zwischen den Linsen L1 und L2 aufgestellt wird. Dadurch verbessert sich die Tiefenschärfe so weit, dass das Gittermuster einigermaßen gut sichtbar ist. Nach erfolgter Justierung wird die Irisblende wieder entfernt. 


\subsubsection{Der Kontinuumsstrahlengang}

In den im Hauptstrahlengang aufgenommenen Filtergrammen ist noch die spektrale Transmissionskurve der Apparatur enthalten. Diese muss gesondert gemessen werden, um sie später aus den Beobachtungsdaten herausrechnen zu können. Hierzu kann über einen beweglichen Spiegel ( $\mathrm{S}_{\mathrm{MM} 1}$ in Abb. 3.2) das Licht einer Kontinuumslampe (KL), das keine Spektrallinien enthält, in den Hauptstrahlengang eingespiegelt werden. Als Kontinuumslampe wird ein Diaprojektor verwendet, weil dieser eine hohe Lichtintensität erzeugt. Dieser Kontinuumsstrahlengang ist so gestaltet, dass er möglichst exakt den Strahlengang des Teleskops einschließlich des Pupillenbilds nachbildet. In diesem Licht wird derselbe Spektralbereich abgetastet wie bei den Beobachtungen mit Sonnenlicht und dabei der Intensitätsverlauf aufgezeichnet. Das Auswerteverfahren wird in Kap. 5.2.2 näher beschrieben.

\subsubsection{Laser- und Photomultiplierstrahlengang}

Um eine möglichst hohe effektive Finesse $\mathcal{F}_{\text {eff }}$ zu erreichen, müssen die Spiegelplatten der FPIs möglichst parallel gestellt sein. Um die Plattenparallelität zu kontrollieren und zu optimieren, werden ein Laserstrahlengang und ein Photomultiplierstrahlengang verwendet (Abb. 3.2). Mittels des beweglichen Spiegels $\mathrm{S}_{\mathrm{MM} 2}$ kann paralleles Laserlicht mit einem Bündeldurchmesser von ca. $2 \mathrm{~cm}$ durch die FPIs gelenkt werden. Anhand der entstehenden Interferenzmuster lässt sich die Parallelität der Platten grob justieren, was beim FPI 2 eine manuelle Einstellung an mechanischen Schrauben erfordert.

Die Feinjustierung erfolgt nicht mehr über eine visuelle Kontrolle der Interferenzmuster. Hierfür wird stattdessen das FPI, das gerade justiert werden soll, wiederholt mit konstanter Geschwindigkeit über einen Wellenlängenbereich von mehreren FSR gefahren. Für das FPI 2 bietet hierfür das Steuergerät eine Möglichkeit (Rampengenerator), für das neue FPI 1 musste mit Hilfe des Steuer-PCs (PC 2) eine Rampe programmiert werden, die dann auf das Steuergerät von FPI1 übertragen werden kann. Der Intensitätsverlauf beim mehrfachen Durchfahren des FSR zeigt immer dann Maxima, wenn das Laserlicht das FPI in aufeinanderfolgenden Ordnungen passieren kann. Da sich in diesem Bereich weniger Ordnungen der Plattenabstand insgesamt prozentual sehr wenig ändert, erhält man somit einen Intensitätsverlauf, der der Airyfunktion hinreichend genau ähnelt. Zeichnet man diesen Intensitätsverlauf auf, so kann aus den gemessenen Werten von FSR und Halbwertsbreite nach Glg. 3.6 die effektive Finesse berechnet werden. Zum Aufzeichnen des Intensitätsverlaufs wird das Licht statt auf die CCD 2 mittels des beweglichen Spiegels $\mathrm{S}_{\mathrm{MM} 3}$ auf einen Photomultiplier (PM) gelenkt. Dessen Ausgangssignal kann mit einem Oszilloskop betrachtet werden. Wenn die Wiederholfrequenz des Abtastens der Intensitätskurve hoch genug ist, ergibt sich auf dem Oszilloskop ein stehendes Bild, in dem leicht die benötigten Größen abgelesen werden können. Durch Drehen von Reglern an den Steuergeräten (Spannung an den Piezokristallen) kann die 
Finesse der FPIs auf ihren maximalen Wert eingestellt werden. Die in Tab. 3.2 angegeben Werte für $\mathcal{F}_{\text {eff }}$ wurden ebenfalls auf diese Weise ermittelt. Dabei muss u. U. noch die absolute Breite der Laserlinie von 2 pm (Bendlin (1993) berücksichtigt werden.

Der Photomultiplierstrahlengang mit dem genannten Messverfahren und dem Oszilloskop kann weiterhin gut dafür verwendet werden, bei Messungen mit Sonnenlicht die Spektrallinien zu identifizieren und das Interferenzfilter IF 2 und die Durchlasswellenlänge von FPI 1 zu justieren.

\subsection{Instrumentelle Polarisation}

Optische Geräte wie Teleskope, Spiegel oder komplette Beobachtungsaufbauten können den Polarisationszustand von Licht in unerwünschter Weise verändern. Dabei kann sowohl der Polarisationsgrad des Lichts verändert werden, also z. B. neues polarisiertes Licht erzeugt werden, es können aber auch die verschiedenen Polarisationsarten des Lichts ineinander umgewandelt werden. Man spricht in diesem Fall vom „Übersprechen“ einer Polarisationsart in eine andere, häufig wird auch das englische Wort "Crosstalk“ verwendet. Diese instrumentelle Polarisation muss bei der Auswertung von polarimetrischen Messdaten wie denen der vorliegenden Arbeit berücksicht werden. Dazu sind aber genaue Kenntnisse der Polarisationseigenschaften der verwendeten Geräte notwendig.

Die Polarisationseigenschaften optischer Geräte werden durch ihre Muellermatrix beschrieben (vgl. Kap. 2.2.3). Für die vorliegenden Beobachtungsdaten wird die instrumentelle Polarisation in erster Linie durch das Teleskop erzeugt, und hier insbesondere an den Coelostatenspiegeln. An diesen treten nicht-senkrechte Reflexionen auf. Dabei gibt es für die transversal zur Ausbreitungsrichtung schwingenden elektromagnetischen Feldvektoren Vorzugsrichtungen, in denen die Reflexion unterschiedliche Eigenschaften haben kann, d. h. die Reflexion ist dann anisotrop. Dies ist nicht der Fall bei (fast) senkrechten Reflexionen oder Transmissionen an oder durch isotrope Stoffe, also an den Ein- und Austrittsfenstern des Vakuumtanks oder am Hauptspiegel.

Für die vorliegenden Messdaten existieren keine genauen Angaben über die Muellermatrix des Beobachtungsaufbaus, da eine direkte Messung der Matrixelemente im Anschluss an die Beobachtungen aus technischen Gründen nicht möglich war. Es existieren aber Messungen aus früheren Jahren (Hofmann (2000), die im Folgenden kurz besprochen werden sollen, weil sie einen Anhaltspunkt geben können über die Größenordnung der zu erwartenden Effekte. Dabei sind mehrere Umstände zu berücksichtigen:

- Die Matrixelemente ändern sich im Laufe des Tags und im Laufe des Jahres wegen der sich ständig ändernden Einfallswinkel des Sonnenlichts auf die beiden Coelostatenspiegel.

- Die Matrixelemente sind abhängig von der gewählten Coelostatenstellung, ebenfalls wegen unterschiedlicher Einfallswinkel auf die Coelostatenspiegel. In Abhängig- 
keit von der Jahreszeit können darüberhinaus noch unterschiedliche Stellungen des ersten Coelostatenspiegels gewählt werden. Insbesondere wurde in den Morgenstunden der Aufnahme der vorliegenden Beobachtungsdaten der erste Coelostatenspiegel in die westliche Stellung gebracht, um eine vollständige Ausleuchtung des Pupillenbilds zu erreichen. Das hat zur Folge, dass größere Einfallswinkel des Sonnenlichts gegen die Spiegeloberfläche auftreten, ähnlich wie zu Beobachtungen um die Mittagszeit. In der Regel resultiert daraus eine geringere instrumentelle Polarisation.

- Die Matrixelemente ändern sich mit jeder Erneuerung der Aluminiumbedampfung der Spiegel, die hin und wieder durchgeführt wird, aufgrund nicht exakt reproduzierbarer Materialeigenschaften der Bedampfung.

- Außer den aus der oben genannten Messung entnommenen Matrixelementen des Teleskops bis zum Primärfokus muss berücksichtigt werden, dass im vorliegenden Beobachtungsaufbau weitere Reflexionen auftreten, die die instrumentelle Polarisation weiter verändern können, z. B. die Reflexion an dem $45^{\circ}$-Umlenkspiegel, der das Licht waagerecht in Richtung des Optischen Labors II bringt.

Mit Kenntnis dieser Umstände sei nun beispielhaft eine Muellermatrix gegeben, wie sie unter den vorliegenden Beobachtungsbedingungen gültig sein könnte. Die Werte dafür sind den oben genannten Messungen aus den Jahren 1993 - 1995 (Hofmann (2000) entnommen.

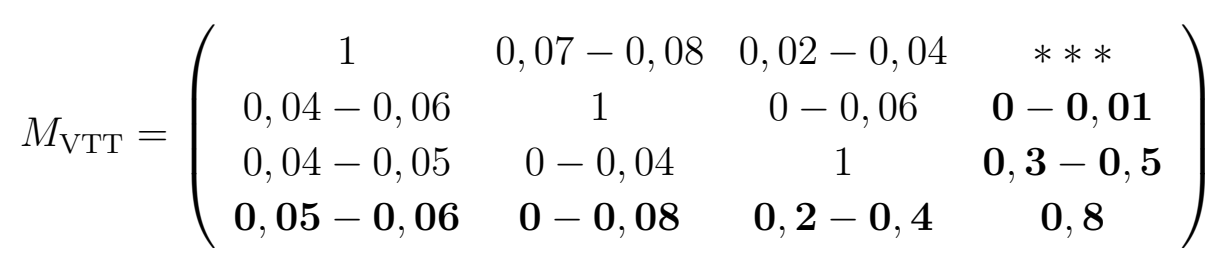

Das Matrixelement $M_{14}$ wurde nicht gemessen. Die für die vorliegenden Beobachtungsdaten wichtigen Matrixelemente sind in Fettdruck wiedergegeben. Zunächst geben die Matrixelemente $M_{24}$ und $M_{34}$ an, wieviel zirkular polarisiertes Licht in linear polarisiertes umgewandelt wird. Dabei wird deutlich, dass ein ganz erheblicher Bruchteil von bis zu 50\% des zirkular polarisierten Lichts von der Sonne verloren gehen kann, der dann hauptsächlich in den Stokes-U-Anteil übertragen wird. Dies bedeutet allerdings lediglich eine Verringerung des gemessenen Stokes-V-Signals.

Größere Schwierigkeiten bereiten die Matrixelemente $M_{41}$ bis $M_{43}$, die das Übersprechen von $I, Q, U \longrightarrow V$ beschreiben. Das Element $M_{41}$ ist noch relativ leicht abzuschätzen, da $I$ die gemessene Gesamtintensität und damit bekannt ist. Nach Glg. 3.8 müsste sich zwischen den beiden Kanälen des Polarimeters ein Differenzsignal von einigen Prozent der Gesamtintensität zeigen, insbesondere müsste sich in dem Differenzbild auch außerhalb der magnetisch sensitiven Spektrallinien deutlich der Intensitätsverlauf, z. B. über Poren und Flecken, widerspiegeln. Das war bei den in dieser Arbeit beschriebenen Daten nicht 
der Fall, ein nennenswerter Beitrag des Matrixelements $M_{41}$ zur instrumentellen Polarisation ist also, im Gegensatz zu den genannten Messungen, bei diesen Beobachtungen nicht nachweisbar.

Die Matrixelemente $M_{42}$ und $M_{43}$ beschreiben das Übersprechen $Q \longrightarrow V$ bzw. $U \longrightarrow V$, wobei letzteres wiederum erhebliche Bruchteile des linear polarisierten Lichts ausmachen kann. Das bedeutet, dass bis zu 40\% des in dem beobachteten Sonnenlicht vorhandenen linear polarisierten Lichts in zirkular polarisiertes umgewandelt werden kann, welches dann durch das Stokes-V-Polarimeter und die dahinterliegende CCD 2 fälschlicherweise registriert würde. Deshalb sind nur in solchen Fällen die gefundenen Messwerte vertrauenswürdig, in denen davon ausgegangen werden kann, dass von vorn herein kein oder nur sehr wenig linear polarisiertes Licht vorhanden ist. Das ist also z. B. bei reinem longitudinalen Zeeman-Effekt bei Beobachtung von einzelnen, senkrecht aus der Sonnenoberfläche austretenden Flussröhren in der Scheibenmitte der Sonne der Fall.

\subsection{Der Beobachtungsablauf}

Zunächst muss die Beobachtungsanlage in einen für die gewünschten Beobachtungen geeigneten Zustand gebracht werden. Es werden Interferenzfilter der geeigneten Wellenlängen eingesetzt, Lage der Bildfelder auf den Kameras und deren richtige Fokusposition überprüft und gegebenenfalls justiert. Für polarimetrische Messungen muss vorher noch das Polarimeter eingesetzt und die bildfeldbegrenzenden Blenden und die Kippung von FPI 1 zur Vermeidung der Reflexbilder entsprechend angepasst werden.

Desweiteren werden die Aufnahmedaten in die Steuer-PCs eingegeben. Die Durchlassbereiche der beiden FPIs müssen aufeinander und auf die gewünschte Spektrallinie abgestimmt werden. Dazu werden Photomultiplierstrahlengang und Oszilloskop verwendet. Die im Detail recht trickreichen Vorgehensweisen mussten, insbesondere für die Einstellung des neuen FPI1, teilweise noch erarbeitet und kontinuierlich verbessert werden.

Wenn alle Einstellungen stimmen und auch die effektiven Finessen der FPIs ein letztes Mal überprüft und eingestellt wurden, kann eine Beobachtungsserie gestartet werden. Die drei Kameras nehmen dann simultan eine bestimmte, vorher zu bestimmende Anzahl $t$ von Bildern auf. In dieser Arbeit wurde mit je fünf Aufnahmen pro Wellenlängenposition gearbeitet, d. h. pro Spannungseinstellung an den Piezoelementen der FPIs. Danach werden die maximalen Transmissionswellenlängen der beiden FPIs um einen ebenfalls frei wählbaren Wert verändert und wiederum dieselbe Anzahl von Einzelbildern aufgenommen. Dieser Vorgang wiederholt sich, bis maximal 140 Einzelbilder (bei vollem Bildfeld) in den Arbeitsspeichern jedes PCs vorliegen. Mit den Durchlassbereichen der FPIs wurde währenddessen normalerweise eine Spektrallinie abgetastet. Eine solche Datenserie wird im Folgenden „Scan“ genannt. Sie enthält also bei CCD 2 für eine bestimmte Anzahl spektraler Positionen (hier meistens 28) eine Anzahl Einzelbilder (hier meistens 5). CCD 1 und 1a enthalten dieselbe Gesamtzahl Einzelaufnahmen, 


\subsection{Der Beobachtungsablauf}

die aber alle breitbandig mit identischer spektraler Verteilung sind. Die Aufnahme eines solchen Scans von insgesamt je 140 Bildern dauert 39 Sekunden.

Während der folgenden 35 Sekunden werden die Scans auf die jeweiligen Festplatten der PCs abgespeichert, und die FPIs werden auf ihre Anfangswellenlänge zurückgesetzt. Nun kann automatisch der nächste Scan gestartet werden, so dass die Gesamtdauer zwischen den Starts zweier Scans 74 Sekunden beträgt. Diese Werte können sich natürlich ändern, wenn andere Bildfeldanteile ausgelesen werden oder die Anzahl der Einzelaufnahmen verändert wird. Darüber hinaus sind inzwischen die PCs durch neuere, leistungsfähigere Modelle ersetzt worden, so dass sich die mögliche Gesamtzahl der in einem Scan aufzunehmenden Bilder vergrößert sowie die Dauer der Scans verringert haben.

Während die PCs die Beobachtungsdaten vom Arbeitsspeicher auf die Festplatten übertragen, hat man Gelegenheit, die Finesse von FPI 2 nachzuregeln oder fotografische Aufnahmen des Pupillenbilds des Teleskops anzufertigen (vgl. Kap. 3.1). Desweiteren muss eine langsame, unregelmässige Drift des Durchlassbereichs von FPI 2 manuell nachgeregelt werden.

Nach Beendigung der Beobachtungsserien müssen verschiedene Datensätze aufgenommen werden, die für die Datenreduktion benötigt werden. Sinn und Verarbeitungsschritte dieser Datenserien werden im Detail später in Kap. 5.1.1 beschrieben. Es werden mit defokussiertem Teleskop „Flatfieldscans“ aufgenommen und in ähnlicher Weise solche mit fokussiertem Teleskop. Hierbei wird aber während der Aufnahmen das Teleskop bewegt, so dass auf den Kameras ein ständig wechselnder Bildausschnitt der Sonnenoberfläche aufgenommen wird. Dabei tasten die FPIs denselben Wellenlängenbereich ab wie bei den Beobachtungsscans. Bei abgedecktem Sonnenlicht werden „Dark-Scans“ aufgenommen, aus denen sich später ein mittleres Dunkelsignal bestimmen lässt. Abschließend wird statt des Sonnenlichts das Licht der Kontinuumslampe in den Strahlengang eingespiegelt, und es werden Kontinuumsscans aufgenommen, in denen das FPI 2 wiederum den Wellenlängenbereich der Beobachtungsscans abtastet. 


\section{Beobachtungen}

Im Laufe mehrerer Beobachtungsaufenthalte wurden viele verschiedene Datensätze mit und ohne Polarimeter aufgenommen. Dabei wurden verschiedenste Objekte von ruhiger, nicht magnetisch aktiver Granulation über Plage-Regionen bis zu Sonnenflecken beobachtet. Bei der Auswertung der Beobachtungsdaten stellte sich heraus, dass für eine erfolgreiche Bildrekonstruktion, die ein hinreichend gutes räumliches Auflösungsvermögen der rekonstruierten Bilder von besser als $1^{\prime \prime}$ ergibt, sehr gutes bis exzellentes Seeing erforderlich ist. Insbesondere müssen die Beobachtungsbedingungen während eines gesamten Scans über eine Spektrallinie (Dauer ca. 40 Sekunden) auf hohem Niveau bleiben, um nicht an einzelnen spektralen Positionen im Scan schlechte Rekonstruktionen zu bekommen. Die in dieser Arbeit vorgestellten Ergebnisse stammen von einem Beobachtungstag mit besonders gutem Seeing, dem 22. 10. 1999.

\subsection{Die Fe I-Linie bei $630,25 \mathrm{~nm}$}

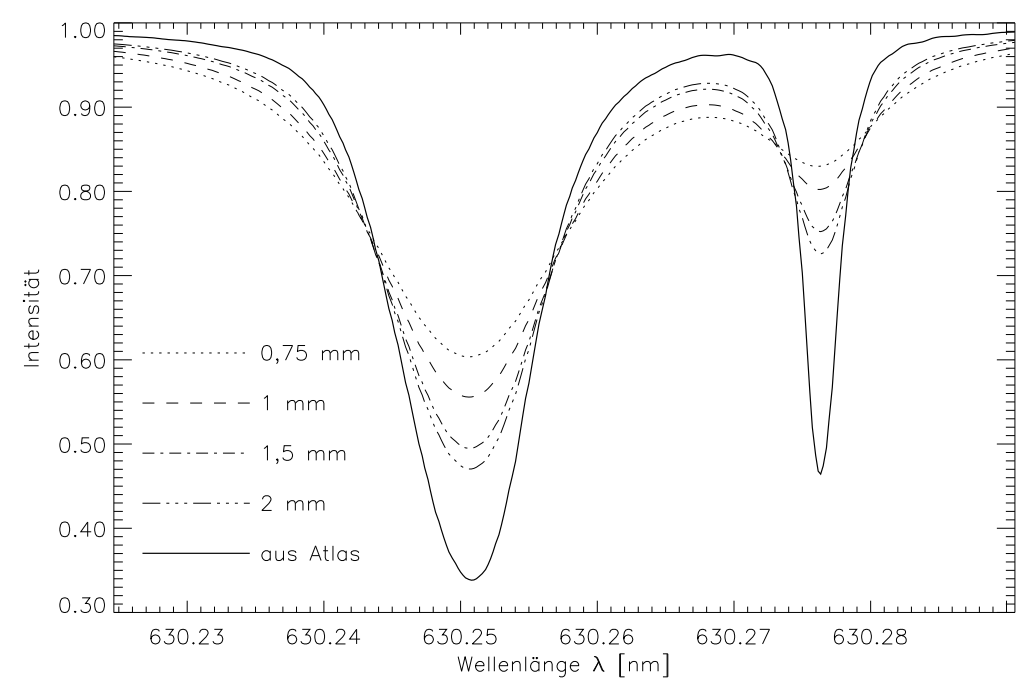

Abbildung 4.1: Linienprofil von Fe I $630,25 \mathrm{~nm}$ und $\mathrm{O}_{2}$ $630,27 \mathrm{~nm}$, aus dem Spektralatlas (durchgezogen) und gefaltet mit Airyfunktionen für verschiedene Plattenabstände von FPI 2.
Um die Ergebnisse der Datenauswertung richtig einschätzen zu können, soll zunächst ein sehr einfaches Bild einer Spektrallinie entworfen werden, an dem die prinzipielle Wirkung von Magnetfeldern und der Einfluss der Messapparatur auf die beobachteten Linienprofile studiert werden können. Es sei betont, dass aus einer solch einfachen Modellvorstellung nur grobe Zusammenhänge entnommen werden können, die aber zum Verständnis ausreichend sind. Als repräsentatives 


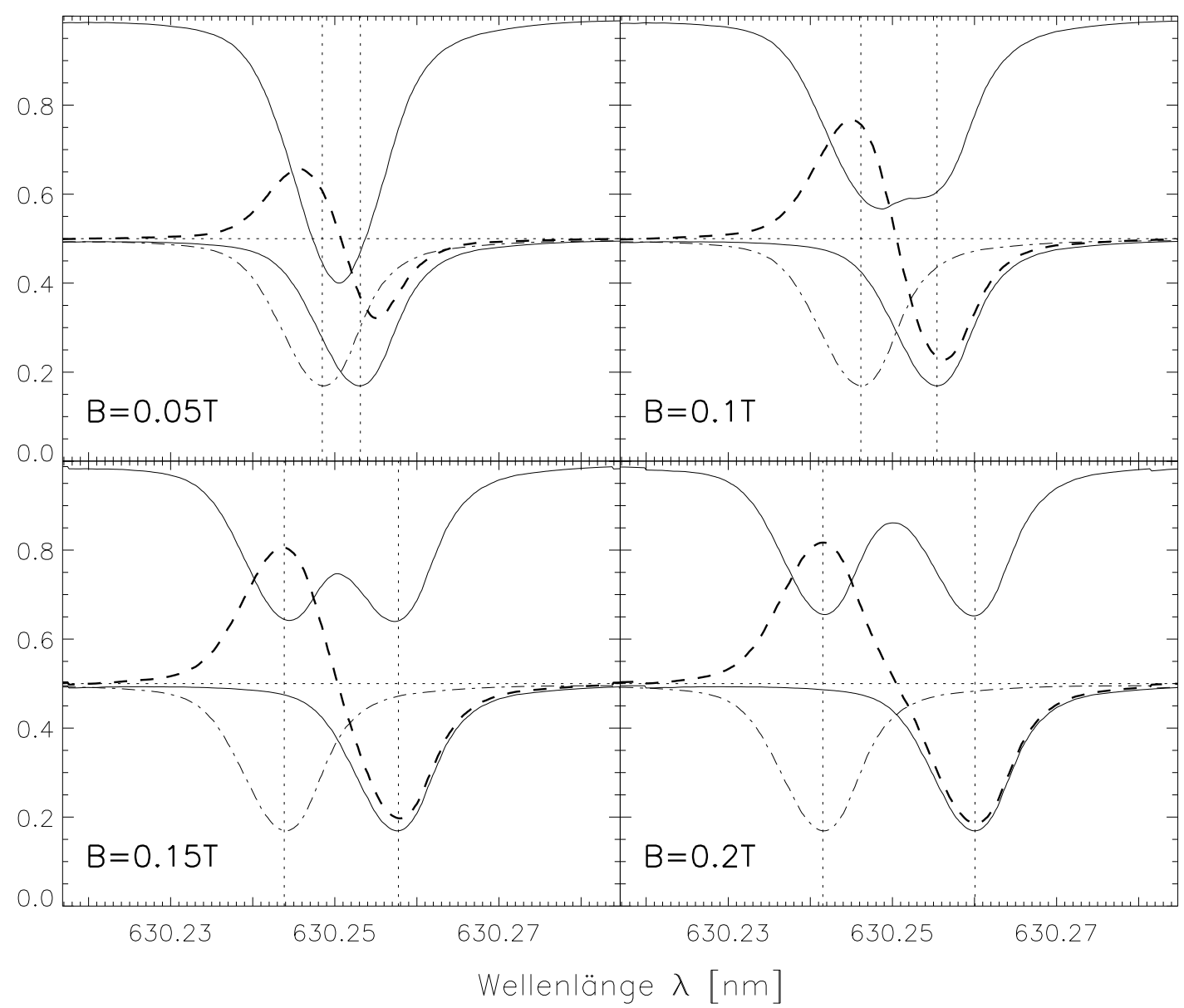

Abbildung 4.2: Stokes-Profile von Fe I 630,25 nm: Gezeigt sind für verschiedene Magnetfelder jeweils das Stokes-I-Profil (obere durchgezogene Linie), die beiden $\sigma$-Komponenten (untere durchgezogene und strichpunktierte Linien), sowie das Stokes-V-Profil (fett gestrichelt). Das VProfil wurde zur besseren Darstellbarkeit um 0,5 nach oben versetzt. Zusätzlich sind gestrichelt das Nullniveau für das V-Profil und die Minima der beiden $\sigma$-Komponenten eingetragen.

Linienprofil wurden Daten aus einem Spektralatlas (Brault \& Neckel (1987) verwendet, die den spektralen Intensitätsverlauf in der mittleren, unmagnetischen Sonnenphotosphäre wiedergeben. Abb. 4.1 zeigt die beiden Linien Fe I (630,25 nm) und $\mathrm{O}_{2}(630,27 \mathrm{~nm})$ aus dem Spektralatlas und nach der Faltung mit Airyfunktionen von FPIs mit verschiedenen Plattenabständen, die verschiedene spektrale Auflösungsvermögen $\lambda / \Delta \lambda$ ergeben. Man erkennt, dass der langwellige Linienflügel der Fe I-Linie nach der Faltung bis in die terrestrische $\mathrm{O}_{2}$-Linie hineinreicht. Dies muss z. B. bei der Anwendung der Linienschwerpunktsmethode (vgl. Kap. 5.2.5) berücksichtigt werden.

Da die $\mathrm{O}_{2}$-Linie bei 630,27 nm erdatmosphärischen Ursprungs ist, hat sie keinen Einfluss auf das in der Sonnenatmosphäre entstehende Linienprofil, und im Idealfall perfekten Flatfieldings auch keinen Einfluss auf das Stokes-V-Signal. Deshalb wurde im Folgenden durch geeignete Interpolation das Linienprofil der Fe I-Linie vom langwelligen Linien- 


\section{Beobachtungen}

flügel bis ins Kontinuum fortgesetzt, die $\mathrm{O}_{2}$-Linie quasi „herausgeschnitten“.

Nun nehmen wir an, dass unter sonst gleichen atmosphärischen Bedingungen (Temperatur, Dichte etc.) ein Magnetfeld einer bestimmten Stärke $B$ unabhängig von der Höhe in der Sonnenatmosphäre parallel zur Sichtlinie vorhanden sei円, und dass das Licht mit einem Stokes-V-Polarimeter beobachtet wird. Die beiden $\sigma$-Komponenten ergeben sich dann durch einfache Verschiebung der Linienprofile gemäß Gleichung 2.4. Durch Subtraktion lässt sich nun das Stokes-V-Profil erzeugen. Abb. 4.2 zeigt die V-Profile bei verschiedenen Feldstärken. Dabei wurde immer ein Füllfaktor $\alpha=1$ angenommen, d. h. dass in $100 \%$ der in einem Auflösungselement beobachteten Fläche der Sonnenatmosphäre das Magnetfeld vorhanden sein soll. Für geringere Füllfaktoren müssen die Intensitäten der $\sigma$-Komponenten und damit auch des V-Profils einfach linear mit $\alpha$ skaliert werden, da die gemessenen Lichtanteile, die aus den nicht magnetischen Flächenanteilen stammen, bei der Bildung der Stokes-V-Profile herausfallen. Die Stokes-I-Profile erhalten dann aber zusätzlich noch eine unverschobene Linienkomponente aus den unmagnetischen Flächenanteilen, die die Aufspaltung der Linie noch undeutlicher werden lässt. Die Asymmetrie der I- und V-Profile stammt hier von der leichten Asymmetrie der Fe I-Linie (C-Shape), wie sie aus dem Spektralatlas entnommen wurde.

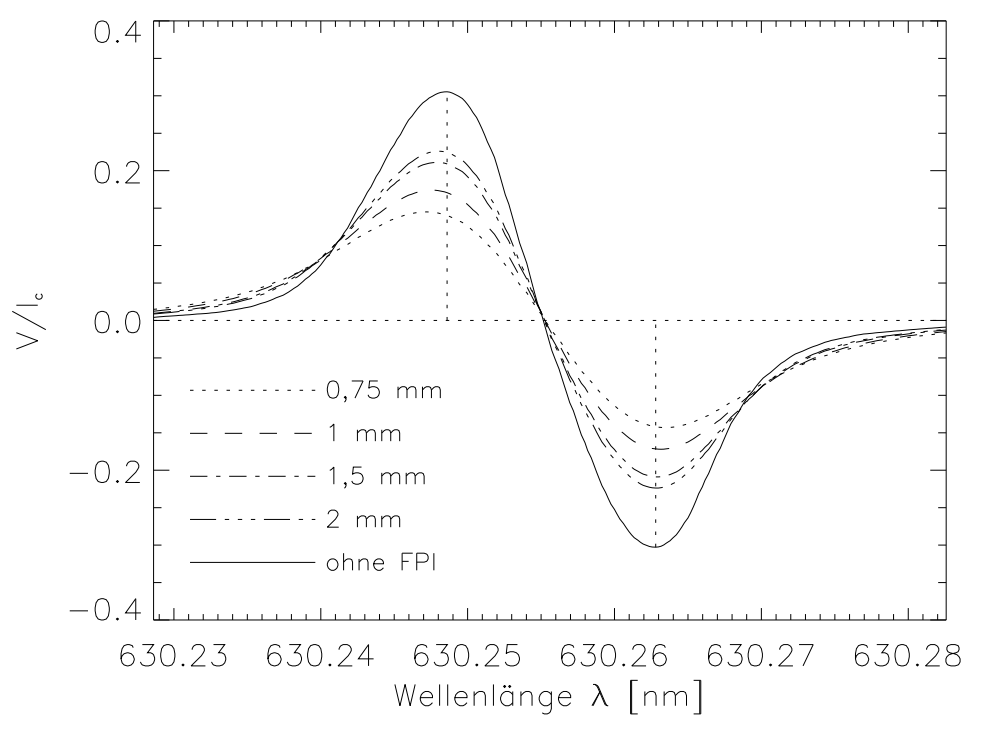

Abbildung 4.3: Stokes-V-Profile von Fe I 630,25 nm (durchgezogen) und nach Faltung mit Airyfunktionen für verschiedene Plattenabstände von FPI 2. Die Positionen der Extrema des ungefalteten V-Profils sind durch gestrichelte Linien markiert.
In Abb. 4.3 ist das Stokes-V-Profil für eine typische Feldstärke von $B=0,15 \mathrm{~T}$ gezeigt, das wiederum mit $\mathrm{Ai}$ ryfunktionen von FPI 2 bei verschiedenen Plattenabständen gefaltet wurde. Es zeigt sich, dass davon im Wesentlichen nur die Amplitude des V-Signals beeinflusst wird, nur sehr schwach dagegen die Trennung der Extrema.

In Abb. 4.4 sind die $\mathrm{Zu}-$ sammenhänge zwischen V-Aufspaltung und Magnetfeld und zwischen V-Amplitude und Mag-

\footnotetext{
${ }^{1}$ Bei genaueren Simulationen zu erwartender Linienprofile ist zu beachten, dass die atmosphärischen Bedingungen in magnetischen Gebieten der Sonnenatmosphäre häufig sehr verschieden sind und mit der Höhe variieren und deshalb auch sehr unterschiedliche Linienprofile erwartet werden.
} 
netfeld dargestellt, im Vergleich zur Aufspaltung der $\sigma$-Komponenten der Linie. Nur für Magnetfelder oberhalb etwa $B=0,15 \mathrm{~T}$ wird die Aufspaltung der $\sigma$-Komponenten gut durch die Aufspaltung des V-Signals wiedergegeben. Dagegen gibt die Amplitude des V-Signals nur für kleine Feldstärken einen guten Hinweis auf die Stärke des Magnetfelds, für stärkere Felder als ungefähr $B=0,1 \mathrm{~T}$ tritt eine Sättigung der V-Amplitude ein. Zudem ist die V-Amplitude noch von dem meist unbekannten Füllfaktor $\alpha$, von den atmosphärischen Größen in den magnetischen Flächenanteilen und vor allen Dingen auch vom Kosinus des Sichtwinkels der magneti-

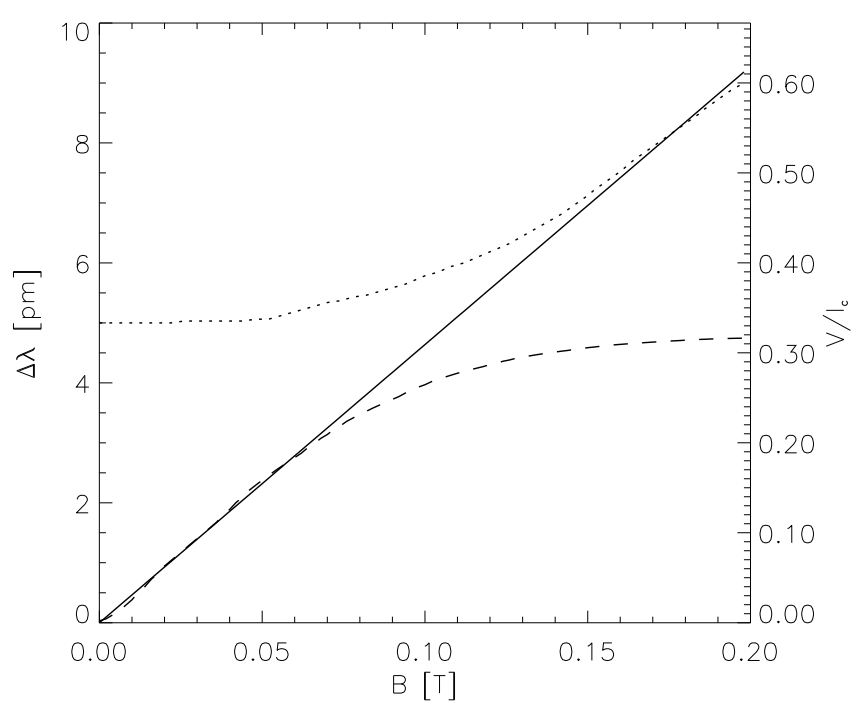

Abbildung 4.4: Aufspaltung der $\sigma$-Komponenten (durchgezogen) und Aufspaltung der Stokes-VExtrema (gepunktet) in Abhängigkeit von der magnetischen Feldstärke $B$. Außerdem ist die StokesV-Amplitude angegeben (gestrichelt). schen Feldlinien abhängig.

Dies alles zeigt, dass Amplitude und Aufspaltung der V-Profile nur grobe Hinweise auf die wahren magnetischen Feldstärken geben, die mit Vorsicht interpretiert werden müssen. Eine andere Methode, die einen zuverlässigen Zusammenhang zwischen magnetischem Fluss und der Aufspaltung der Linienschwerpunkte von $\frac{1}{2}(I+V)$ und $\frac{1}{2}(I-V)$ ergibt, wird später in Kapitel 5.2 .5 vorgestellt.

\subsection{Beobachtungen am 22.10. 1999}

An diesem Tag sind die meisten brauchbaren Beobachtungsdaten bei teilweise exzellentem Seeing entstanden. Zu Kalibrations- und Testzwecken wurde von 9:51 - 9:56 UT eine kurze Zeitserie von vier Scans in einem magnetisch sehr ruhigen Gebiet mit Granulation in der Scheibenmitte aufgenommen. Auch in diesen Scans wurden kleine, schwach magnetische Strukturen entdeckt, die in Kap. 6 genauer besprochen werden sollen. Das Magnetogramm von diesem Tage vom Kitt Peak NSO ([1]) (Abb. 4.5) zeigt in der Scheibenmitte keine nennenswerte Aktivität, die ungefähre Lage des Beobachtungsgebiets ist durch das kleinere Rechteck markiert.

Weiterhin wurde die bipolare Gruppe NOAA 8738 nahe der Scheibenmitte $(\mu=0,97)$ | beobachtet. Gute Zeitserien mit einer Länge von je 20 Scans wurden von dem vorange-

\footnotetext{
${ }^{2} \mu=\cos \theta$ mit $\sin \theta=$ Verhältnis des Abstands von der Scheibenmitte zu Sonnenradius
} 


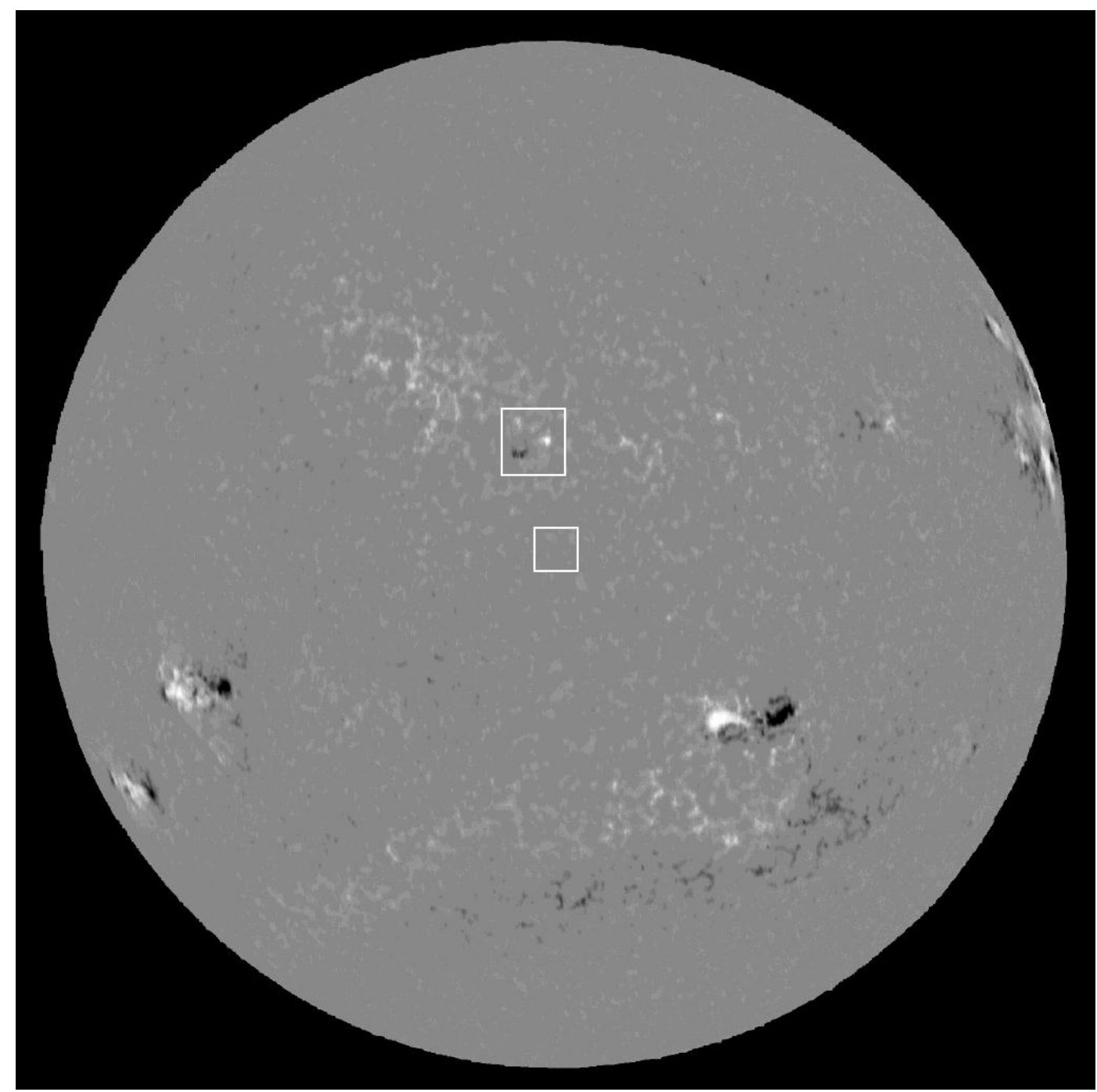

Abbildung 4.5: Magnetogramm vom Kitt Peak NSO vom 22.10. 99. Durch Rechtecke markiert sind die Region der beobachteten ruhigen Granulation in der Scheibenmitte und darüber die aktive Region NOAA 8738.

henden, kleinen Sonnenfleck dieser Gruppe aufgenommen, der Ansätze einer Penumbra zeigt (Abb. 4.6). Ebenfalls konnten Zeitserien von der nachfolgenden Porengruppe erhalten werden (Abb. 2.1), die mehrere kleine Poren und anomale Granulation enthält. Die genauen Beobachtungsdaten und Bezeichnungen der Scans sind Tabelle 4.1 zu entnehmen. Einzelne Scans einer Serie werden im Folgenden z. B. in der Form s5_4 angegeben — für den 4. Scan der 5. Serie.

Sobald wie möglich nach Beendigung der Aufnahmen (ca. 11:00 UT) wurde mittels eines in den Primärfokus gestellten Fotoapparats die aktive Gruppe auf Schwarzweißfilm (TP 2415) aufgenommen, um später die kleinen Bildfelder der CCD-Aufnahmen räumlich einander zuordnen und eventuelle Schlüsse bei der Interpretation der Beobachtungs- 


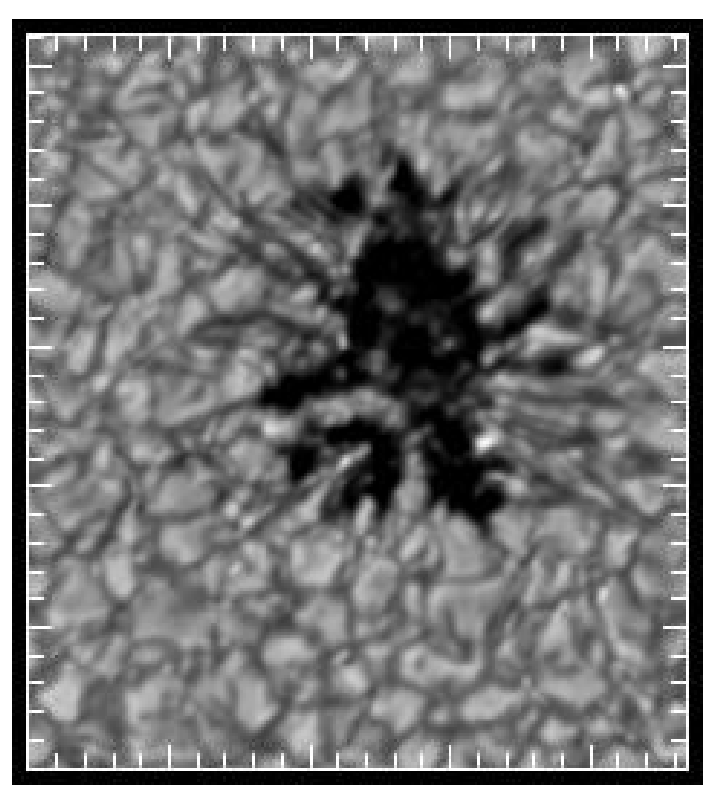

Abbildung 4.6: Kleiner Sonnenfleck aus den Beobachtungen vom 22.10.99. Es handelt sich hierbei um den vorangehenden Teil der bipolaren aktiven Region NOAA 8738. Der Abstand der Strichmarken entspricht $1^{\prime \prime}$.

\begin{tabular}{|c||c|c|c|l|c|}
\hline Serie & Anzahl Scans & Uhrzeit (UT) & $\Delta t[\mathrm{~ms}]$ & Objekt & $r_{0}[\mathrm{~cm}]$ \\
\hline \hline s1 & 20 & $8: 13-8: 38$ & 30 & kl. Fleck, NOAA 8738 & $8-10$ \\
\hline s2 & 20 & $8: 45-9: 09$ & 30 & Porengruppe, NOAA 8738 & $8-11$ \\
\hline s3 & 10 & $9: 10-9: 22$ & 20 & Porengruppe, NOAA 8738 & $10-12$ \\
\hline s4 & 20 & $9: 26-9: 50$ & 20 & kl. Fleck, NOAA 8738 & $11-14$ \\
\hline s5 & 4 & $9: 52-9: 57$ & 20 & Granulation, Scheibenmitte & $12-13$ \\
\hline s6 & 20 & $10: 01-10: 26$ & 20 & Porengruppe, NOAA 8738 & $11-15$ \\
\hline s7 & 5 & $10: 27-10: 33$ & 20 & Porengruppe, NOAA 8738 & $11-12$ \\
\hline
\end{tabular}

Tabelle 4.1: Beobachtungsdaten vom 22.10.99. Angegeben sind die Namen der Serien, die Anzahl der Scans pro Serie, Uhrzeit des Aufnahmezeitraums, Belichtungszeit, Art des beobachteten Objekts und der Friedparameter $r_{0}$ als Parameter für die Qualität des Seeings.

ergebnisse ziehen zu können. Abb. 4.7 zeigt diese Aufnahme, in der die ungefähre Lage der Bildausschnitte der Aufnahmeserien s4 und s6 markiert ist. Da sich innerhalb von 30 - 60 Minuten nicht nur die Granulation, sondern auch die Struktur der Poren und des kleinen Sonnenflecks merklich verändert hat, ist eine exakte Zuordnung der Ausschnitte nicht möglich. Der Bildausschnitt dieses Fotos hat eine Größe von 90" $\times 130^{\prime \prime}$, die Belichtungszeit ist $8 \mathrm{~ms}$. Abgeschwächt wurde die Intensität durch ein ca. $10 \mathrm{~nm}$ breites Interferenzfilter $(557 \mathrm{~nm})$ und ein $3 \mathrm{~mm}$ starkes Neutralfilter NG 5 .

Weiterhin wurden aus den verfügbaren Quellen im Internet Aufnahmen anderer Obser- 


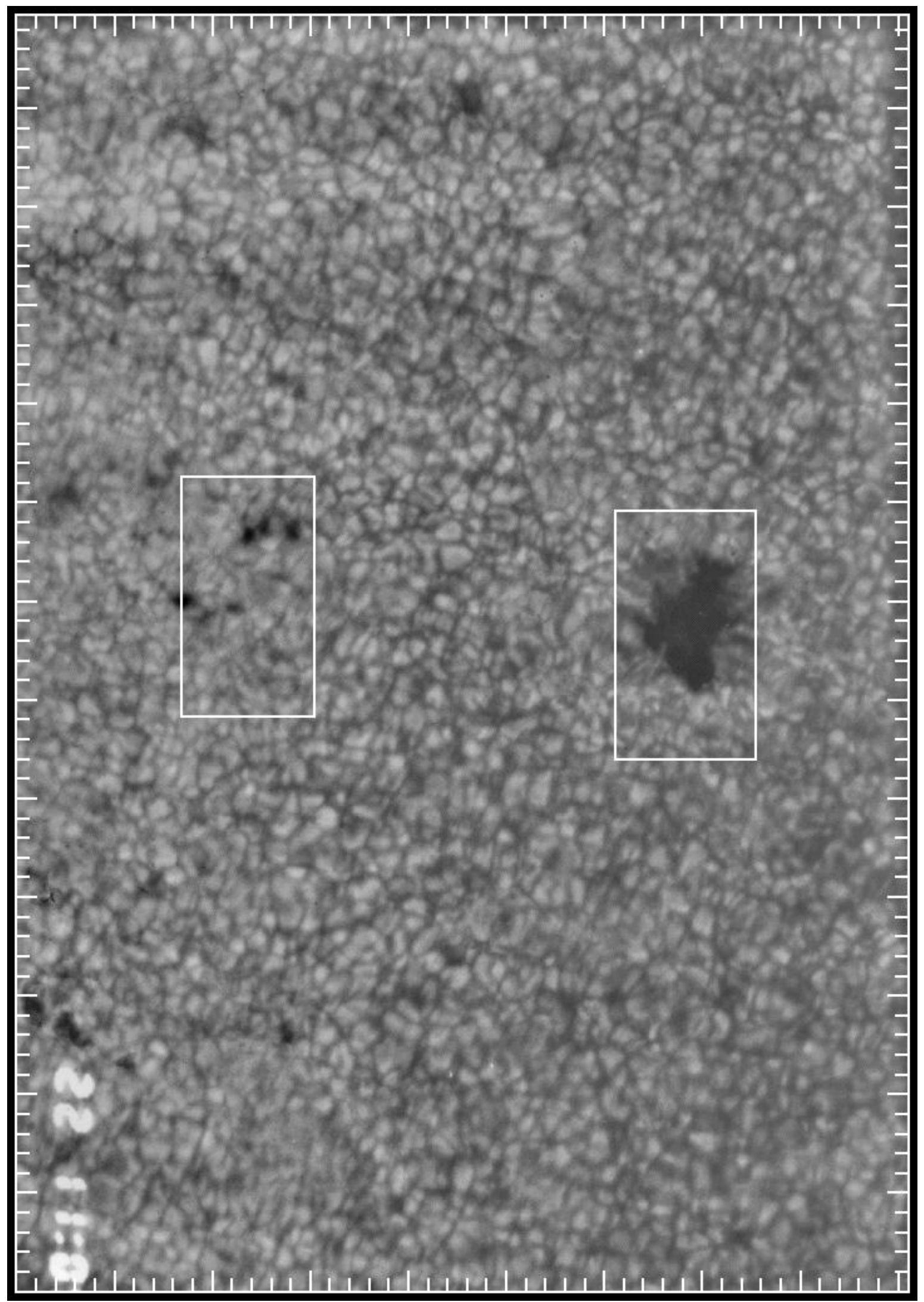

Abbildung 4.7: Foto von NOAA 8738. Markiert sind die mit den CCD-Kameras aufgenommenen Ausschnitte der Scans s4 und s6. Der Abstand der Strichmarken entspricht 2". 
vatorien dieser aktiven Region gesammelt, um eine möglichst umfassende Interpretation der eigenen Beobachtungsergebnisse zu ermöglichen. Dazu gehört das schon erwähnte Magnetogramm vom Kitt Peak NSO (Abb.4.5, [1]), auf dem die markierte aktive Region etwas nordöstlich der Scheibenmitte gut zu erkennen ist.

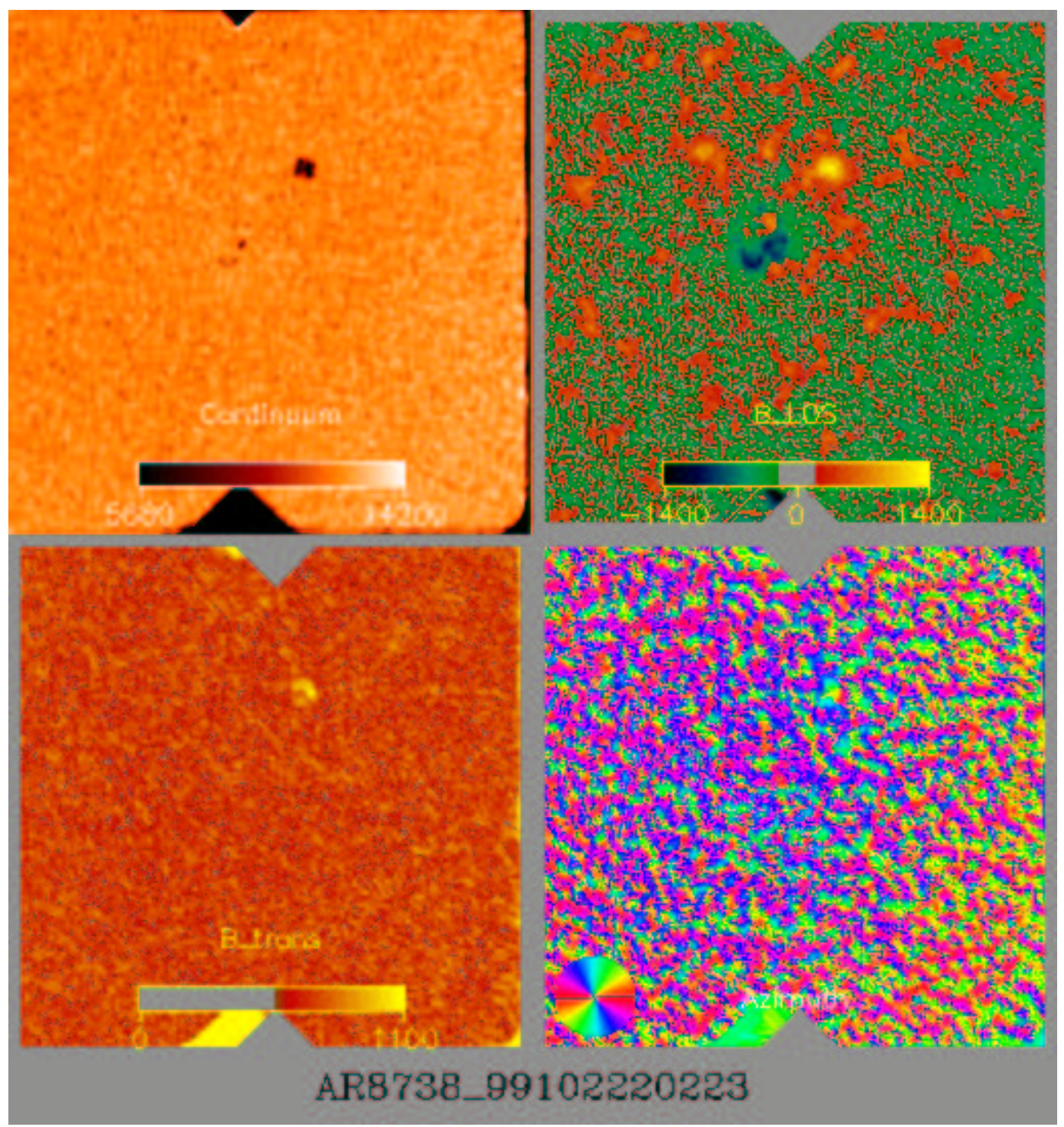

Abbildung 4.8: Aufnahmen von NOAA 8738 vom Mees Solar Observatory, Hawaii vom 22.10.99.

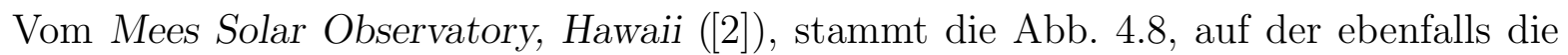
aktive Region NOAA 8738 beobachtet wurde. Im Kontinuumsbild (oben links) sind der kleine Sonnenfleck und die Porengruppe zu erkennen, im Magnetogramm der Sichtlinienkomponente (oben rechts) sind die unterschiedlichen Polaritäten sichtbar. Das Magnetogramm der transversalen Magnetfeldkomponente (unten links) zeigt nur für den kleinen Sonnenfleck ein merkliches Signal. Dies hat nicht unwesentliche Bedeutung für 


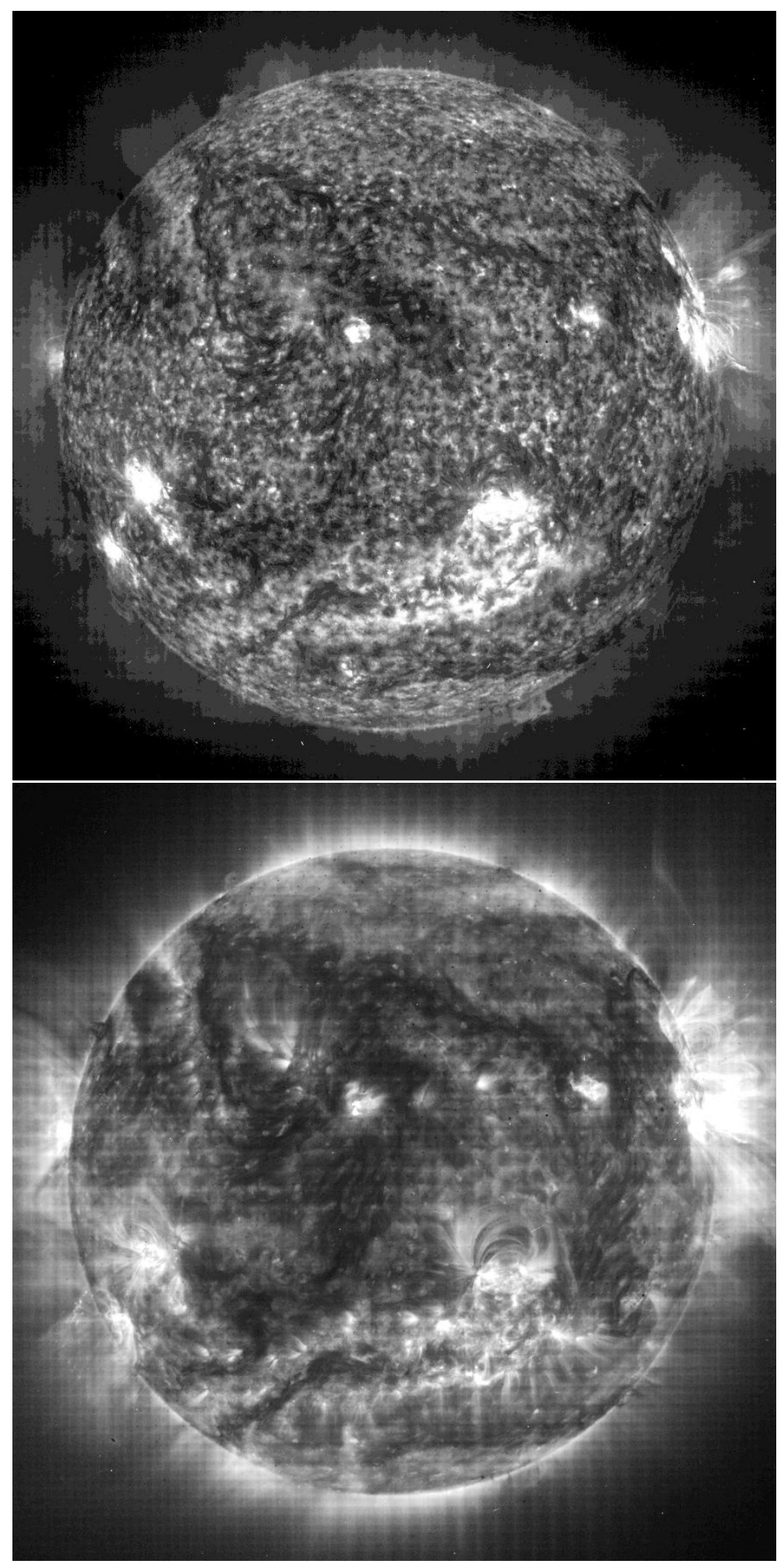

Abbildung 4.9: EIT-Bilder der Sonne am 22.10.99. Oben bei $\lambda=30,4 \mathrm{~nm}$, unten bei $\lambda=$ $17,1 \mathrm{~nm}$. 
die spätere Interpretation der Messergebnisse in Kap. 6.3. Die Auftragung der gemessenen Feld-Azimuths (unten rechts) zeigt i. W. Rauschen.

Abb. 4.9 wurde von der Raumsonde SOHO (Solar and Heliospheric Observatory) mit dem Instrument EIT (Extreme ultraviolet Imaging Telescope) aufgenommen ([3]). Die obere Aufnahme zeigt die Übergangsschicht der Sonne im Licht einer Heliumlinie (He II) bei einer Wellenlänge von 30,4 nm, die einem Temperaturbereich von 60000-80000 K entspricht. Die heißen Bereiche der Korona (ca. $10^{6} \mathrm{~K}$ ) werden in der unteren Aufnahme sichtbar, die im Licht zweier Eisenlinien (Fe Ix/X) bei einer Wellenlänge von 17,1 nm entstanden ist. Beide Aufnahmen zeigen links oberhalb der Bildmitte das kleine Aktivitätsgebiet, das aber keine weiteren Besonderheiten aufweist. Genau in der Mitte der Sonnenscheibe, wo die Granulationsscans s_5 aufgenommen wurden, ist bis auf das chromosphärische Netzwerk keine Aktivität zu finden.

Schließlich zeigt Abb. 4.10 entsprechende Ausschnitte aus synoptischen Beobachtungen vom Big Bear Solar Observatory (BBSO, 44) im Licht der Spektrallinien $\mathrm{CaK}$ und $\mathrm{H} \alpha$. Ein genauerer Vergleich mit den eigenen Messergebnissen folgt später in Kap. 6 und 7. Eine Liste der verwendeten Internetadressen wird im Anhang an das Literaturverzeichnis gegeben.

An dieser Stelle sei außerdem darauf hingewiesen, dass in den Bildern, die den entsprechenden Internetseiten entnommen sind (4.5, 4.8-4.10), wie üblich rechts We-

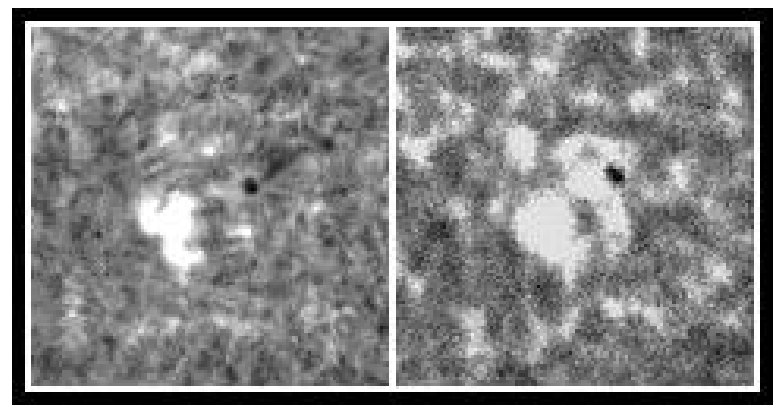

Abbildung 4.10: Aufnahmen von NOAA 8738 vom Big Bear Solar Observatory vom 22.10.99. Links ist die Region im Licht der $\mathrm{H} \alpha$-Linie aufgenommen, rechts im Licht der Ca K-Linie. sten und oben Norden ist, jedoch in allen im Laufe dieser Arbeit selbst aufgenommenen Bildern (also sowohl in dem Foto 4.7 als auch in sämtlichen CCD-Bildern, Magnet- und Geschwindigkeitskarten) Westen ebenfalls rechts, Norden aber unten ist.

Für diesen Tag wurden folgende Einstellungen der Beobachtungsanlage gewählt: Beobachtet wurde die Spektrallinie Fe I bei $630,25 \mathrm{~nm}$, der Plattenabstand von FPI 2 war $d_{2}=$ $1 \mathrm{~mm}$. Daraus ergibt sich eine Halbwertsbreite der Durchlasskurve von $\Delta \lambda=5,6 \mathrm{pm}$ (Tab. 3.2), die einem spektralen Aufösungsvermögen von $\lambda / \Delta \lambda \approx 1,2 \cdot 10^{5}$ entspricht. Das ist nicht so viel, wie bei vergleichbaren Beobachtungen mit Gitterspektrografen erreicht werden kann. Dafür ist die über den Durchlassbereich des FPIs integrierte Lichtintensität hoch genug, dass mit hinreichend kurzen Belichtungszeiten gearbeitet werden kann, um später Bildrekonstruktionsmethoden anwenden zu können. Im Verlaufe des Morgens konnte die Belichtungszeit von $30 \mathrm{~ms}$ auf $20 \mathrm{~ms}$ verkürzt werden. Das verwendete Vorfilter IF 2 (Abb. 3.2) hat eine Halbwertsbreite von 1,1 nm und im Bereich der 


\section{Beobachtungen}

beobachteten Linie eine fast konstante Transmission von $46 \%$.

Pro Scan wurden mit CCD 2 an 28 Wellenlängenpositionen je fünf Bilder aufgenommen, so dass ein Scan insgesamt 140 Bilder umfasst. Mit einem Abstand zweier FPI-Positionen von 2,7 pm ergibt sich ein insgesamt abgetasteter Wellenlängenbereich von $73 \mathrm{pm}$. Die Aufnahme von 140 Bildern dauerte 39 s, das anschließende Abspeichern auf Festplatte weitere $35 \mathrm{~s}$, so dass der zeitliche Abstand zwischen dem Beginn zweier Scans einer Serie insgesamt $74 \mathrm{~s}$ beträgt.

Der 1. Coelostatenspiegel des Teleskops wurde in die West-Stellung gebracht (vgl. Kap. 32), um schon kurz nach Sonnenaufgang eine möglichst volle Ausleuchtung der Eintrittspupille zu erreichen und möglichst hohe Lichtintensität zu haben. Um später die Form der Eintrittspupille kontrollieren und eventuell in die Datenauswertung einbeziehen zu können, wurden in unregelmäßigen Abständen zwischen den Serien Aufnahmen von der Eintrittspupille auf Schwarzweißfilm angefertigt.

Nach Beendigung der Beobachtungen wurden verschiedene Aufnahmen zur Datenkalibration gemacht: Dunkelbilder und Flatfieldscans, außerdem Scans mit dem Licht der Kontinuumslampe. Die Finesse von FPI 2 wurde während der Beobachtungen und der Kalibrationsaufnahmen mehrfach kontrolliert und gegebenenfalls nachgeregelt. 


\section{Datenverarbeitung}

Die Verarbeitung der Beobachtungsdaten gliedert sich in zwei wesentliche Abschnitte. Zunächst wird mit Methoden der Bildrekonstruktion versucht, aus den bei jeder Wellenlängenposition aufgenommenen Einzelbildern soviel Information wie möglich zu gewinnen und daraus ein möglichst gutes, repräsentatives Bild an dieser Position zu berechnen. Das Ergebnis sollte dann ein Datenkubus sein, der mit maximaler Auflösung zwei Raumdimensionen und eine Wellenlängendimension enthält. Im zweiten Schritt wird dann für jeden Punkt der beiden Raumdimensionen die Wellenlängeninformation, also das Spektrum, analysiert. Dabei werden in erster Linie die Magnetfelder bestimmt, aber auch Informationen über Geschwindigkeiten, Höhenschichtung der Atmosphäre, Intensitäten usw. ermittelt.

\subsection{Bildrekonstruktion}

In diesem Abschnitt sollen verschieden aufwendige, aber auch verschieden erfolgreiche Methoden der Bildverarbeitung und Bildrekonstruktion vorgestellt werden. Dazu soll gleichzeitig kurz die Theorie erläutert und am Beispiel die Wirkungsweise und die Ergebnisse der Methoden gezeigt werden. Aufgrund der völlig verschiedenen Rauschniveaus von breit- und schmalbandigen Daten unterscheiden sich auch die Maßnahmen der Bildrekonstruktion voneinander. Insbesondere beruht das Prinzip der vorgestellten Arbeit darauf, leicht rekonstruierbare, rauscharme Breitbandbilder (im Folgenden auch „CCD 1- und CCD 1a-Bilder" genannt) mit etablierten Rekonstruktionsmethoden zu verarbeiten und so quantitative Informationen über die während der simultanen Aufnahme eines Bildersatzes aus CCD 1-, CCD 1a- und CCD 2-Bildern vorliegende Optische Transferfunktion (OTF) zu bekommen. Diese Informationen sollen im nächsten Schritt eine Rekonstruktion der schmalbandigen Bilder (im Folgenden „CCD 2-Bilder“) ermöglichen. Die folgenden Unterkapitel beschreiben die nötigen Vorarbeiten an den drei Datensätzen und verschiedene Rekonstruktionsmethoden. Je nach Methode werden die Ergebnisse an den entsprechenden Datensätzen gezeigt. Da das eigentliche Interesse den schmalbandigen Daten gilt, die die spektroskopische Information enthalten, widmet sich die Beschreibung vorwiegend diesen Bildern. Auf die Verarbeitung der breitbandigen CCD 1-Bilder soll nur insoweit eingegangen werden, wie es für das Verständnis der Verarbeitung der CCD 2-Bilder notwendig ist. 


\subsubsection{Vorverarbeitung der Daten}

Vor der eigentlichen Bildrekonstruktion müssen bestimmte grundlegende Verarbeitungsschritte durchgeführt werden, wie sie bei den meisten astrophysikalischen Arbeiten an Beobachtungsdaten üblich sind. Andere Verarbeitungsschritte sind spezifisch für den verwendeten Aufbau und die Art der aufgenommenen Daten.

\section{Drehen der Bilder und Bestimmen der gemeinsamen Bildausschnitte}

Als erstes werden die CCD 2- und die CCD 1a-Bilder so gedreht und gespiegelt, dass sie die gleiche Bildorientierung wie die CCD 1-Bilder haben. Zunächst ist diese unterschiedlich, weil die drei Aufnahmen unterschiedliche Spiegelungen an den Strahlteilerwürfeln erfahren, und die CCD 2-Bilder außerdem eine Abbildung durchlaufen und dabei gespiegelt werden (vgl. Kap. [3). Dann müssen die gewünschten (zumeist größtmöglichen) Ausschnitte der beiden Polarisationskanäle aus den CCD 2-Bildern herausgeschnitten werden, wobei natürlich darauf geachtet werden muss, dass sich die abgebildeten Gebiete der Sonnenoberfläche exakt entsprechen. Derselbe Ausschnitt muss aus den CCD 1und CCD 1a-Daten gewählt werden. Zum Bestimmen der korrekten Ausschnitte wurden Kreuzkorrelationsmethoden verwendet, die im Fourierraum leicht anzuwenden sind. Dabei wurden beim Vergleich von CCD 2- mit CCD 1-Daten immer Bildausschnitte aus solchen CCD 2-Bildern gewählt, die im Kontinuum aufgenommen wurden, da sich schmalbandige Bilder im Linienkern von mittelstarken oder starken Spektrallinien erheblich von Kontinuumsbildern unterscheiden können.

\section{Dunkelbildkorrektur}

Neben den durch einfallendes Licht erzeugten Elektronen in den Pixeln der CCD-Kamera werden auch durch thermische Anregung ständig Elektronen in die Leitungsbänder des Halbleitermaterials gehoben, die sich zusätzlich in den Pixeln des CCD-Chips ansammeln. Die Anzahl der am Ende einer Aufnahme zusätzlich angesammelten Elektronen (und damit die vom Analog-Digital-Wandler registrierte zusätzliche Zählrate) hängt ab von

- der Temperatur des Chips,

- der Dauer der Belichtung und des zeilenweisen Auslesens der Aufnahme,

- einer über die Ausleseelektronik des Steuergeräts der Kamera eingestellten Vorspannung („Offset“) und

- den individuellen Eigenschaften eines jeden Pixels.

Um die Gesamtgröße des Dunkelsignals möglichst gering zu halten und so den dynamischen Bereich der Kamera voll ausnutzen zu können, wird der Chip soweit wie möglich 


\subsection{Bildrekonstruktion}

mittels Peltierelementen gekühlt (wobei darauf geachtet werden muss, dass das Eintrittsfenster der Kamera nicht beschlägt), und die Vorspannung wurde im Vorfeld der Beobachtungen so eingestellt, dass bei den gewünschten Temperaturen und Belichtungszeiten das Dunkelsignal so niedrig wie möglich, aber in keinem Bildelement null ist.

Zusätzlich kann noch ein unerwünschtes Falschsignal durch Streulicht aus dem Beobachtungsraum erzeugt werden, welches seitlich in die Beobachtungsanlage eindringen kann und auf die Kameras fällt. Um den Einfluss des verbleibenden Dunkelsignals minimieren zu können, wurden unter sonst gleichen Bedingungen kurze Scans aufgenommen, bei denen das Sonnenlicht abgedeckt wurde. Diese Scans werden gemittelt und unter Beachtung der richtigen Bildausschnitte von den jeweiligen Datenscans abgezogen.

\section{Flatfieldkorrektur}

Wenn man mit der Beobachtungsanlage eine völlig gleichmäßig beleuchtete Fläche aufnehmen würde, sollte man auch ein völlig gleichmäßiges Bild auf allen CCDs erwarten. Dies ist in der Praxis nicht der Fall, die Gründe sind

- Abschattungen (Vignettierungen) des Strahlengangs durch den optischen Aufbau,

- Staubkörner und andere Verschmutzungen auf CCD-Chip, Linsen oder anderen optischen Flächen,

- die unterschiedliche Empfindlichkeit der einzelnen Pixel aufgrund von Fertigungstoleranzen des Chips,

- Interferenzstreifen bei schmalbandigen Beobachtungen und

- bei CCD 2 zusätzlich die über das Bildfeld unterschiedlich variierende Transmission für Licht bestimmter Wellenlängen der beiden gegeneinander gekippten FPIs im parallelen Strahlengang (vgl. Kap. 3.2.2).

Um auch diese in den Beobachtungsdaten enthaltenen Störungen minimieren zu können, werden sog. „Flatfieldscans" von einem möglichst strukturlosen, gleichmäßig beleuchteten Objekt (dem „Flatfield“) aufgenommen, die ausschließlich diese Störeinflüsse und das Dunkelsignal enthalten. Als Flatfield kann bei Sonnenbeobachtungen das defokussierte Bild der Sonne ungefähr in Scheibenmitte verwendet werden, sofern sich in diesem Gebiet keine auffälligen oder magnetischen Strukturen befinden. Um das statistische Photonenrauschen in diesen Aufnahmen zu minimieren, werden viele Einzelbilder dieser Scans gemittelt. Nach Abzug des Dunkelsignals werden diese Bilder „Verstärkungsmatrix" oder auch "gain table" genannt. Die Beobachtungsdaten werden durch diese Verstärkungsmatrizen dividiert, wodurch die unerwünschten Störeinflüsse, die auch in den Beobachtungsdaten vorhanden sind, herausfallen. Zwei Besonderheiten sind bei den in dieser Arbeit aufgenommenen Daten zu beachten, die eine „Sonderbehandlung“ der 
Aufnahmen erforderten:

a) Bei CCD 1 und CCD 1a befanden sich am 22.10.99 einige sehr dunkle, kontrastreiche und scharf begrenzte Staubkörner auf dem CCD-Chip, deren Schatten zunächst aus den Bildern nicht entfernt werden konnten. Da sich beim Defokussieren des Teleskops die Strahlenganggeometrie etwas verändert, ist die Beleuchtung und damit der Schattenwurf der Staubkörner auf den Chip in den fokussierten Beobachtungsdaten etwas verschieden von dem in den Flatfieldaufnahmen. Deshalb blieben nach der Flatfieldkorrektur geringe Reste von den Staubkörnern sichtbar, die besonders bei der folgenden Bildrekonstruktion Probleme bereiteten. Abhilfe wurde geschaffen, indem möglichst unterschiedliche Einzelaufnahmen aus den fokussierten Beobachtungsscans dieser Kameras gemittelt wurden, und zwar so viele, wie von diesem Beobachtungstag verfügbar waren. Durch die Mittelung gehen die feinen, sich auf der Sonnenoberfläche ständig verändernden, solaren Strukturen wie gewünscht verloren. Da aber an diesem Tag vorwiegend Poren und ein kleiner Sonnenfleck beobachtet wurden, verblieben großräumige Intensitätstrends in der gemittelten Aufnahme. Es wurden deshalb um die Staubkörner herum kleine Gebiete ausgeschnitten und der grobe Intensitätsverlauf an denjenigen in den defokussierten Aufnahmen angepasst. Die Bestimmung des Intensitätsverlaufs erfolgte durch Anpassen einer linearen Regressionsfläche an den Ausschnitt unter Ausschluss des eigentlichen, dunklen Schattens des Staubkorns. Diese kleinen Ausschnitte wurden dann an den richtigen Stellen in das gemittelte, defokussierte Flatfield eingesetzt. Das so erzeugte, einem Flickenteppich ähnliche Flatfield zeigt nur noch sehr geringe Intensitätssprünge an den Kanten der eingefügten Ausschnitte, die weit unterhalb des durch die Photonenstatistik dominierten Rauschens liegen. Die hiermit bearbeiteten Beobachtungsdaten zeigen keine Reste von Staubkörnern mehr. Für spätere Beobachtungsaufenthalte empfiehlt es sich, von vornherein weitere Flatfieldscans mit fokussiertem Teleskop aufzunehmen, während denen das beobachtete Bildfeld ständig verändert wird.

b) Bei CCD 2 ändert sich die Ausleuchtung des Bildfelds während eines Scans. Dies liegt vor allem daran, dass die Wellenlänge maximaler Transmission wegen der Aufstellung der FPIs im parallelen Licht über das Bildfeld variiert. Intensitätsgradienten in Wellenlängenrichtung machen sich deshalb als Intensitätsgradienten im Bildfeld bemerkbar. Auch Interferenzringe sind von der Durchlasswellenlänge der FPIs abhängig und ändern sich geringfügig. Deshalb werden die FPIs auch während der Flatfieldscans über den entsprechenden Wellenlängenausschnitt der Spektrallinie gefahren. Dann wird für jede Wellenlängenposition der FPIs eine eigene Verstärkungsmatrix berechnet, indem die Einzelbilder aller Flatfieldscans an dieser Position und evtl. benachbarter Positionen gemittelt werden. Im Fall der hier besprochenen Daten handelt es sich meistens um 10 - 30 gemittelte Einzelbilder. Da die CCD 2-Daten noch die spektrale Information enthalten, die durch das Flatfielding nicht entfernt oder verändert werden soll, werden die einzelnen Verstärkungsmatrizen auf einen Mittelwert von eins normiert. Dann wird jedes einzelne Bild der Beobachtungsdaten mit eben jener Verstärkungsmatrix reduziert, die seiner Wellenlängenposition im Scan entspricht. 


\subsection{Bildrekonstruktion}

\subsubsection{Korrektur der Bildverschiebung}

Durch Seeing sind die einzelnen Aufnahmen desselben Objekts um bis zu mehrere Bogensekunden gegeneinander verschoben („,image motion“, vgl. Kap. 2.3). Um diese groben Verschiebungen zu korrigieren, werden zunächst sämtliche Einzelbilder von CCD 1 auf das Bild mit dem höchsten Kontrast korreliert. Entsprechend den ermittelten Verschiebewerten wird dann aus jedem Bild der größte gemeinsame Bildausschnitt herausgeschnitten und abgespeichert. Dadurch ergibt sich eine leichte Verkleinerung des Bildfelds um einige Bogensekunden. Da die Bildbewegung nur durch Seeing erzeugt wird, ist sie für alle CCDs identisch. Deshalb werden dieselben Verschiebewerte auch auf die CCD 1aund CCD 2-Bilder angewendet.

\subsubsection{Frameselection}

Eine Methode, möglichst gutes Datenmaterial zu erhalten, die man nicht im eigentlichen Sinne „Bildrekonstruktion“ nennen kann, ist das Heraussuchen des jeweils besten Bilds aus einer bestimmten Auswahl. Sie liefert aber in diesem Fall einen guten Anhaltspunkt für die Informationen, die ohne echte Bildrekonstruktion aus den Daten gewonnen werden können. Insbesondere liefert sie sichere untere Grenzen für die gemessenen physikalischen Größen. Während man bei rekonstruierten Daten von vornherein nie ganz sicher sein kann, ob man nicht bestimmte Strukturen in den Bildern fälschlicherweise „erzeugt" hat, z. B. indem Rauschen verstärkt wurde, sind die Informationen, die sich in den nicht rekonstruierten Bildern aus den unteren Detektionsgrenzen herausheben, als

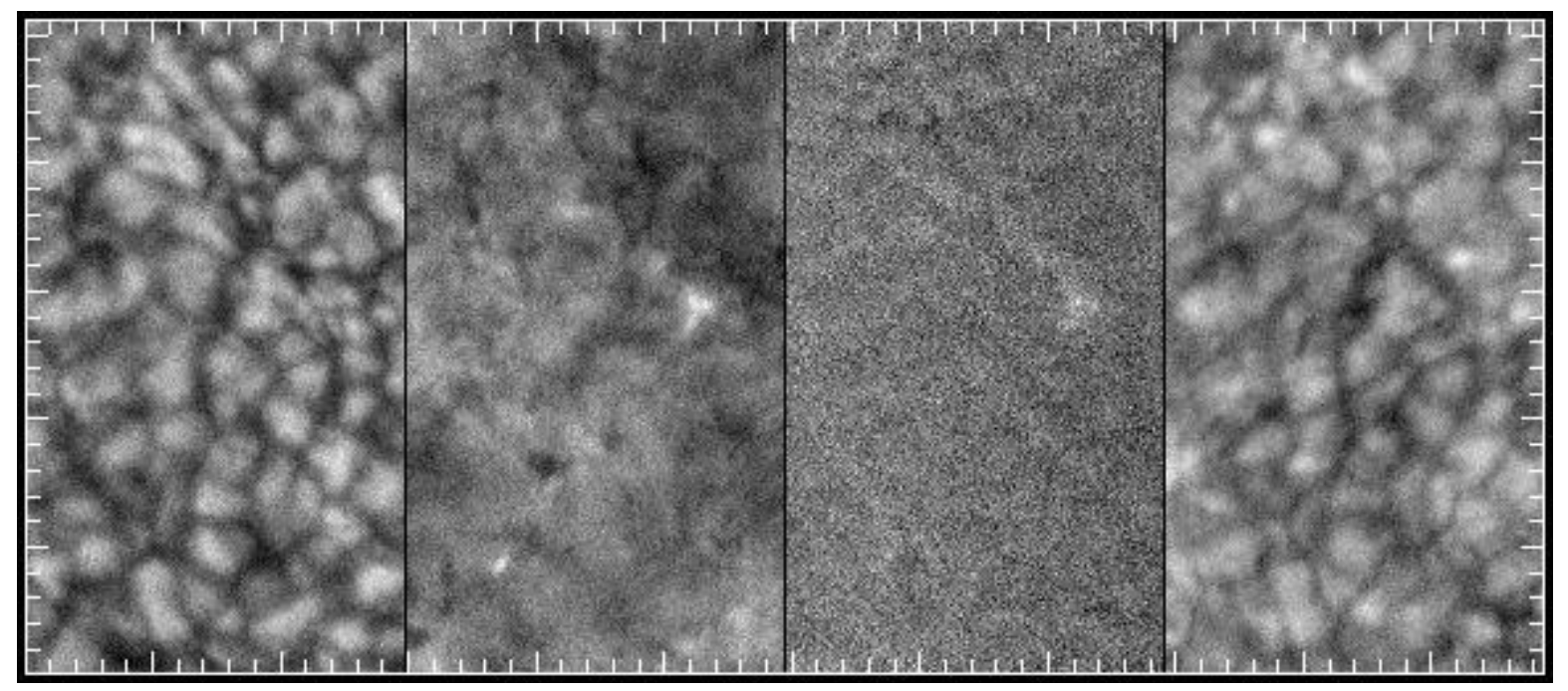

Abbildung 5.1: Ergebnisse der Frameselection-Methode bei Scan s5_4. Gezeigt sind von links nach rechts CCD 2-Bilder aus dem Kontinuum und dem Linienkern, die Magnetfeldkarte und das Geschwindigkeitsbild. Der Abstand der Strichmarken entspricht 1". 


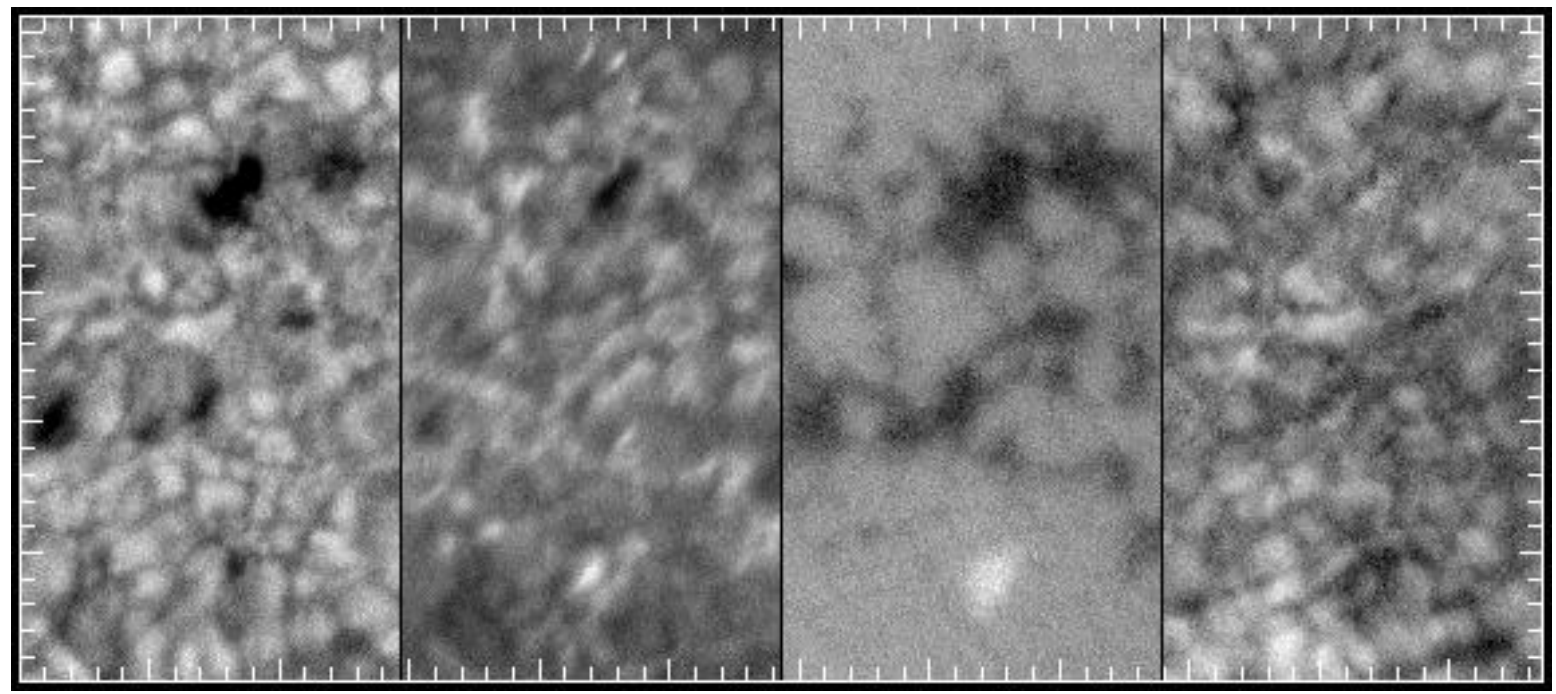

Abbildung 5.2: Ergebnisse der Frameselection-Methode bei Scan s6_6. Gezeigt sind von links nach rechts CCD 2-Bilder aus dem Kontinuum und dem Linienkern, die Magnetfeldkarte und das Geschwindigkeitsbild. Der Abstand der Strichmarken entspricht 1".

untere Grenzwerte zu betrachten.

In diesem Fall stehen an jeder Wellenlängenposition eines Scans jeweils fünf einzelne Realisationen des beobachteten Objekts von verschiedener Seeingqualität zur Verfügung. Als Qualitätsindikator kann der RMS-Kontrast (für Route Mean Square, $\widehat{=}$ Standardabweichung) der Intensität in den CCD 1-Bildern dienen, die Granulation enthalten. Je besser das Seeing ist und je höher die räumliche Auflösung, um so höher ist auch der Intensitätskontrast der sichtbaren Strukturen, wie sich durch visuelle Kontrolle leicht überprüfen lässt. Einige so ausgewählte Einzelbilder von CCD 2 sind in Abb. 5.1 (Scan s5_4) und 5.2 (Scan s6_6) gezeigt. Ebenfalls sind die Magnetfeld- und Geschwindigkeitskarten der betreffenden Ausschnitte dargestellt. Die Bestimmung der Magnetfelder und Geschwindigkeiten wird in Kap. 5.2.5 genauer beschrieben. Die erzielte räumliche Auflösung in den Magnetfeldkarten ist hierbei etwa $1^{\prime \prime}-1,5^{\prime \prime}$. Die Magnetflüsse erreichen bis zu $3,7 \cdot 10^{8} \mathrm{~Wb}$ in einem Pixel in der großen Pore oben in s6_6. Es sei angemerkt, dass es Wellenlängenpositionen gibt, an denen die CCD 2-Bilder noch von deutlich schlechterer Seeingqualität sind als hier dargestellt. Ein genauerer Vergleich dieser Bilder und der physikalischen Messgrößen mit den Ergebnissen von Bildrekonstruktionen wird später in Kap. 5.1.9 erfolgen.

Diese Art der Datengewinnung ist nicht der eigentliche Zweck des aufgebauten Spektrometers und dient hier lediglich dem Vergleich. Sicherlich lassen sich aber auch mit dieser Methode recht gute Datensätze erhalten, wenn das Seeing exzellent ist und die technischen Voraussetzungen gegeben sind. Insbesondere muss die Gelegenheit bestehen, in sehr kurzer Zeit sehr viele Einzelbilder aufnehmen zu können, aus denen das 


\subsection{Bildrekonstruktion}

jeweils beste gespeichert und später verwendet werden kann. Ein begrenzender Faktor für diese Art der Anwendung (zweidimensionale Spektroskopie) ist aber sicherlich die Gesamtdauer eines Scans über eine Spektrallinie. Bei hoher zeitlicher Aufnahmerate der Scans (gewünscht sind Zeiten von weniger als einer Minute pro Scan) verbleiben für jede einzelne Wellenlängenposition nicht mehr als wenige Sekunden, während derer das Seeing die Aufnahme eines exzellenten Bilds mit einer räumlichen Aufösung unterhalb einer Bogensekunde ermöglichen muss. Und auch wenn das gegeben ist, verbleiben noch großräumige Verzerrungen in den Bildern, welche durch „Destretching-Algorithmen“ beseitigt werden müssen, ehe die Bilder aller Wellenlängenposition zu einem gemeinsamen Datenblock hoher Auflösung kombiniert werden können.

\subsubsection{Aufteilen der Bilder in Teilbilder, Fouriertransformation und Zusammensetzen der Teilbilder}

Alle im Folgenden beschriebenen Methoden der Bildrekonstruktion stützen sich auf Verarbeitungsschritte im Fourierraum. Zudem ist zu beachten, dass die Seeingbedingungen sich über das Bildfeld hinweg kontinuierlich ändern, somit auch die zu korrigierenden Effekte auf die beobachteten Bilder. Die Gebiete, über die hinweg das Seeing als im Rahmen der Methode hinreichend konstant angesehen werden kann, werden ,isoplanare Gebiete“ genannt. Die Größe dieser Gebiete hängt sowohl von der verwendeten Wellenlänge (steigt mit $\lambda$ ) als auch vom Seeing (steigt mit besserem Seeing) ab, und wird hier mit $5^{\prime \prime}-6^{\prime \prime}$ angenommen. Aus rechentechnischen Gründen werden die vorverarbeiteten Rohbilder in Teilbilder von $64 \times 64$ Pixel Größe zerlegt $\left(\widehat{=} 6,4^{\prime \prime} \times 6,4^{\prime \prime}\right)$. Auf diese werden dann getrennt die folgenden Maßnahmen der Bildrekonstruktion angewendet. Um weitere Maßnahmen (Apodisation) und das spätere Zusammensetzen der rekonstruierten Teilbilder zu ermöglichen, werden die Teilbildserien mit einem hinreichend großen Überlapp (20 - 30 Pixel) ausgeschnitten.

Da die Einzelbilder noch seeingbedingte und deshalb (zeitlich) veränderliche großräumige Verzerrungen aufweisen können, entsprechen Ausschnitte an gleichen Stellen zweier Bilder nicht unbedingt exakt gleichen Bildfeldern der Sonnenoberfläche. Deshalb werden auch hier Kreuzkorrelationsmethoden angewendet, um für jede Teilbildserie exakt gleiche Bildausschnitte aus den Rohbildern herauszuschneiden. Wie in Kap. 5.1.2 werden die Verschiebewerte anhand der CCD 1-Daten bezüglich des Bilds mit dem höchsten Kontrast errechnet und dann auf die Datensätze aller drei Kameras angewendet. Hierbei ist zu beachten, dass verschiedene Beobachtungsobjekte wie Granulation oder Poren u. U. eine etwas unterschiedliche Berechnung der Kreuzkorrelationen der Ausschnitte erfordern. So kann es z. B. sinnvoll sein, vorher die feinen Bildstrukturen durch „unscharfe Maskierung“ etwas zu verstärken, in anderen Fällen kann eine Beschränkung lediglich auf die groben Intensitätsverläufe im Bild günstig sein. Im Zweifelsfall muss der Erfolg der Verschiebungen durch einen Konsistenztest überprüft werden: Man bestimme die 
Verschiebung zweier Teilbilder $b$ und $c$ bezüglich eines Bilds $a$, dann überprüfe man, ob die Verschiebung von $b$ bezüglich $c$ mit diesen Werten konsistent ist. Durch solche Überprüfungen vieler Bildkombinationen kann man die beste Methode der Kreuzkorrelationsbestimmung erhalten. Die auftretenden Fehler bei nicht optimaler Berechnung der Bildverschiebungen betragen erfahrungsgemäß 1 - 2 Pixel.

Da sich lineare Gradienten über diskrete Datenfelder im Fourierraum nicht darstellen lassen, wird vor der Fouriertransformation von jedem Teilbild eine lineare Regressionsfläche abgezogen, die z. B. bei dem Übergang Granulation $\longrightarrow$ Pore erheblich sein kann. Damit geht auch die 0. Fourierkomponente, das ist die mittlere Intensität des Bilds, verloren. Man kann jedoch davon ausgehen, dass die großräumigen Strukturen vom Seeing kaum beeinflusst werden. Deshalb dürfen die Regressionsflächen abgespeichert und nach erfolgter Bildrekonstruktion einfach wieder aufaddiert werden. Weiterhin setzt die Fouriertransformation endlicher Datenfelder voraus, dass sich der Datensatz periodisch fortsetzen lässt. Damit hierbei keine scharfen Intensitätsgradienten („Kanten“) auftreten, werden die Bilder über einen gewissen Bereich (7 Pixel) am Rand „sanft“ auf einen gemeinsamen Wert gebracht („apodisiert“), am besten auf den Mittelwert des Bilds, hier also null.

Für die anschließende Fouriertransformation wird eine vorgefertigte FFT-Routine (Fast Fourier Transform) des verwendeten Bild- und Datenverarbeitungsprogramms IDL (Interactive Data Language) angewendet. Bei expliziten Darstellungen von optischen Transferfunktionen (OTFs, vgl. auch Kap. 2.3) im Fourierraum ist auf die richtigen Maßstäbe zu achten. Am unkompliziertesten sind die Darstellungen und Umrechnungen der Raumfrequenzen, wenn die Samplingrate der Bilder so gewählt ist, dass die Nyquistfrequenz

$s_{\text {Nyquist }}$ gerade der Grenzfrequenz des Aufösungsvermögens des Teleskops $s_{\text {grenz }}$ entspricht. Dies ist im vorliegenden Fall nicht exakt erfüllt, der Abbildungsmaßstab ist bei einer Wellenlänge $\lambda=630,25 \mathrm{~nm}$ gerade 1,8 Pixel/Auflösungselement.

Nach erfolgter Bildrekonstruktion und Rücktransformation in den Ortsraum werden die vorher abgezogenen, linearen Regressionsflächen und damit auch die mittleren Intensitäten wieder auf die Teilbilder addiert. Diese werden dann pixelgenau wieder zusammengesetzt, wobei die überlappenden Gebiete durch geeignete Gewichtungsmaßnahmen stetig und ohne Kanten ineinander übergeführt werden. Die fertig rekonstruierten und zusammengesetzten Bilder an den einzelnen Wellenlängenpositionen stehen dann für die Bestimmung der spektroskopischen Information und somit der physikalischen Parameter zur Verfügung.

\subsubsection{Entfaltung mit der idealen Teleskopfunktion und Rauschfilterung}

Die Optische Transferfunktion (OTF), die die Abbildungseigenschaften einer jeden einzelnen Aufnahme einer Datenserie beschreibt, setzt sich aus verschiedenen Anteilen zu- 


\subsection{Bildrekonstruktion}

sammen: dem atmosphärischen Seeing, den Abbildungseigenschaften des Teleskops und denen der Postfokusinstrumentierung (vgl. Kap. 2.3). In diesem Ansatz der Bildrekonstruktion soll nur der Anteil des Teleskops an der OTF korrigiert werden. Dabei soll das Teleskop als ideal angesehen werden ohne zusätzliche Abbildungsfehler wie Astigmatismus oder Ähnliches. Die theoretische OTF des Teleskops ist als einziger Beitrag zur gesamten OTF des abbildenden Systems genau bekannt und stellt somit eine untere Grenze für die die räumliche Aufösung begrenzenden Abbildungsfehler dar. Folglich werden die Abbildungsfehler von Atmosphäre, Postfokusinstrumentierung und Teleskop bei der Rekonstruktion unberücksichtigt bleiben. Der Vorteil ist aber, dass man sicher sein kann, für bestimmte Strukturen keine zu große Verstärkung durchgeführt zu haben. Lediglich das in den Beobachtungen enthaltene Rauschen muss mit Vorsicht behandelt werden.

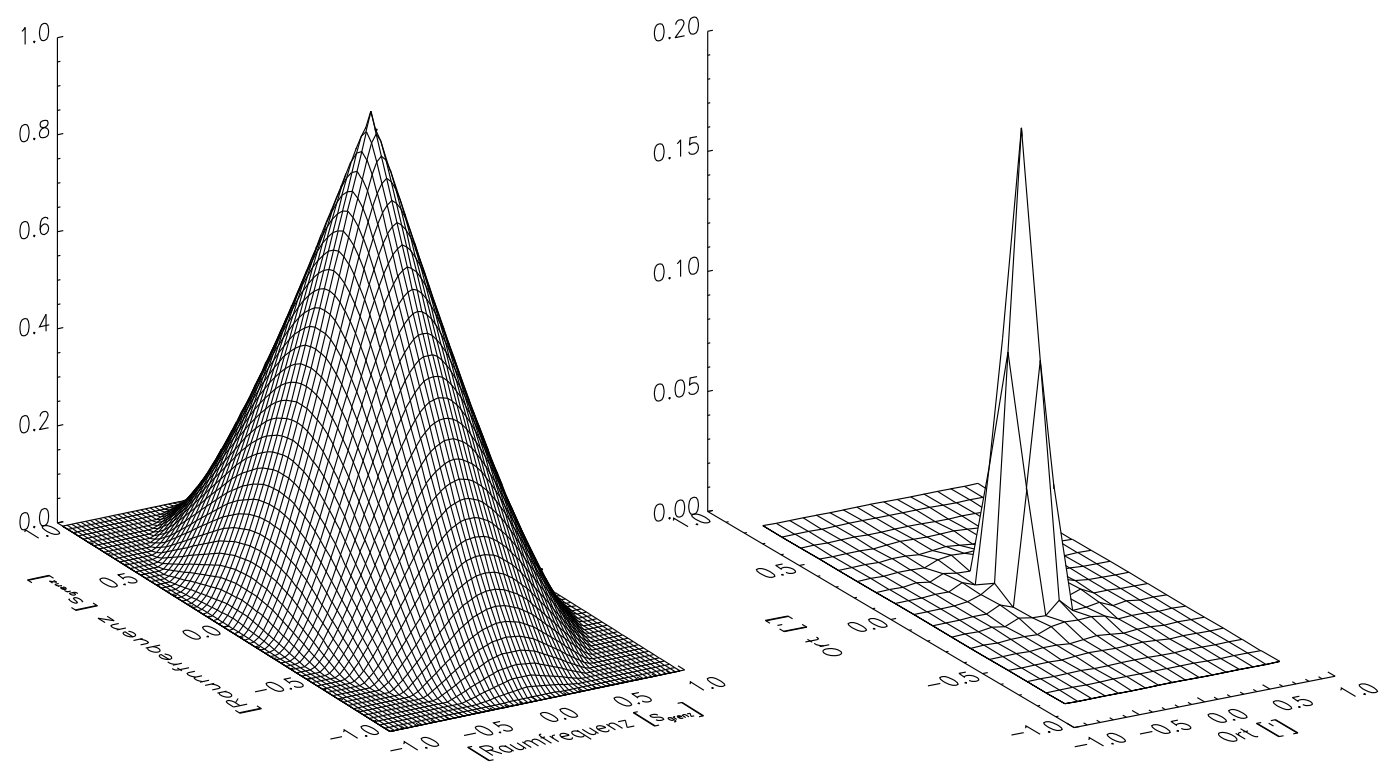

Abbildung 5.3: Links die Optische Transferfunktion (OTF) des idealen Teleskops ohne Abbildungsfehler. Die Raumfrequenzachse ist auf die Grenzfrequenz $s_{\text {grenz }}$ normiert. Rechts die zugehörige Punktverbreiterungsfunktion (PSF).

Die OTF und ein Ausschnitt des zentralen Teils der PSF des idealen Teleskops sind in Abb. 5.3 gezeigt. Die OTF fällt monoton und fast linear bis zur Aufösungsgrenzfrequenz $s_{\text {grenz }}=\lambda / D$ auf null ab. Sie ist rotationssymmetrisch. Die PSF ist ebenfalls rotationssymmetrisch und zeigt darüber hinaus sehr schwach den ersten Beugungsring nach Airy.

Bei der Bildrekonstruktion wird auf Grundlage von Glg. 2.17 eine einfache Entfaltung der beobachteten Bilder mit der OTF durchgeführt, d. h. die in dem Bild enthaltenen Strukturen werden entsprechend ihrer Größe mit dem reziproken Wert der OTF bei 
dieser Raumfrequenz verstärkt. Da oberhalb bestimmter Raumfrequenzen das eigentliche Signal nicht mehr vom immer im Bild vorhandenen Rauschen zu unterscheiden ist, muss hier zusätzlich ein Rauschfilter $H$ verwendet werden, das später in Kap. 5.1.7 (Seite 78) genauer beschrieben werden soll. Damit ergibt sich eine Schätzung des rekonstruierten Bilds folgendermaßen:

$$
\widehat{O}=H \cdot \frac{I_{t} \cdot S^{*}}{|S|^{2}}
$$

Der * bedeutet dabei das konjugiert Komplexe einer Größe. Der Index $t$ mache deutlich, dass es sich um den Zustand einer zeitlich veränderlichen Größe zu einem bestimmten Zeitpunkt $t$ handelt, so bezeichne z. B. $I_{t}$ eines von fünf an einer Wellenlängenposition aufgenommenen Bildern. Im Folgenden sollen sich alle Funktionen im Fourierraum immer auf die Koordinaten $\vec{s}$ beziehen, auch wenn sie nicht mehr explizit genannt werden. Von einer Schätzung wird hier gesprochen, weil natürlich bestimmte Anteile der gesamten OTF unberücksichtigt geblieben sind, folglich das Ergebnis nur eine möglichst genaue, aber nicht exakte Wiedergabe des wahren Objekts darstellt.

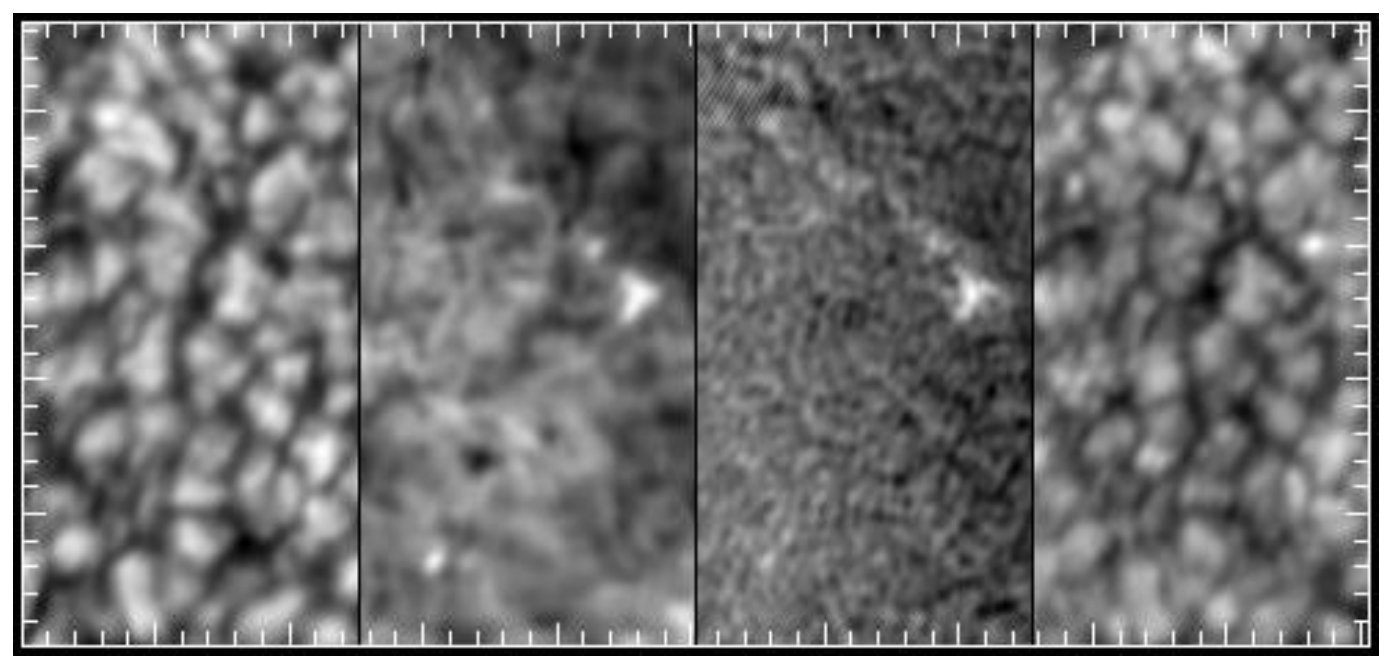

Abbildung 5.4: Ergebnisse der Entfaltung mit der Teleskopfunktion bei Scan s5_4. Gezeigt sind von links nach rechts CCD 2-Bilder aus dem Kontinuum und dem Linienkern, die Magnetfeldkarte und das Geschwindigkeitsbild. Der Abstand der Strichmarken entspricht 1".

Da diese Rekonstruktionen noch starke Verzerrungen innerhalb des Bilds aufweisen, werden alle Rekonstruktionen an einer Wellenlängenposition der FPIs gemittelt. Dadurch ergeben sich bessere magnetische Signale, wenn die Rekonstruktionen zu einem Datenkubus mit spektralen Informationen zusammengesetzt werden. Das Ergebnis einer solchen Bildbearbeitung zeigt Abb. 5.4.

Da, wie auch aus Abb. 5.3 ersichtlich ist, die OTF bis zur Grenzfrequenz beinahe linear von eins auf null abfällt, werden über den allergrößten Raumfrequenzbereich nur sehr moderate Verstärkungen der Kontraste erreicht. Erst für Strukturen von 0,25", das ent- 


\subsection{Bildrekonstruktion}

spricht etwa $0,8 \cdot s_{\text {grenz }}$, erreicht die OTF einen Wert von etwa 0,1 , ist also die Verstärkung der Amplitude der Strukturen etwa zehnfach, und nur für noch kleinere Strukturen ist sie noch höher. Bei solch kleinen Strukturen greift aber im vorliegenden Fall längst das Rauschfilter. Das bedeutet, dass die Verbesserung der Bildqualität und der Magnetfeldkarten im Vergleich zu den Ergebnissen aus den Rohbildern bzw. der FrameselectionMethode in erster Linie auf die Rauschfilterung zurückzuführen sein dürfte, und weniger auf den strukturverstärkenden Effekt der Entfaltung mit der Teleskopfunktion.

\subsubsection{Phase Diversity}

Einen ähnlichen Weg der Bildrekonstruktion geht man bei einem Verfahren, das „FokusVolumen-Methode“ oder „Phase Diversity“ genannt wird. Wesentlicher Bestandteil dieses Verfahrens ist aber, dass die tatsächliche, vollständige OTF inklusive aller Einflüsse des atmosphärischen Seeings, des nicht perfekten Teleskops und Teilen der Postfokusinstrumentierung vorher auf eine bestimmte Weise aus den Messwerten bestimmt wird. Dadurch soll eine möglichst vollständige Rekonstruktion des wahren Beobachtungsobjekts ermöglicht werden. Dabei ist darauf zu achten, dass sich die OTF und damit das beobachtete Bild während der Belichtungszeit nicht verändern, also die Bedingungen für „eingefrorenes Seeing“" erfüllt sind (vgl. Kap. 2.3).

Die OTF kann leicht aus der Form der Wellenfront $\phi$ des in das Teleskop eintretenden Lichts berechnet werden. Eine Möglichkeit wäre, die Form der Wellenfront z. B. mit Hilfe eines Hartmann-Shack-Sensors während der Beobachtung zu messen. Beim Phase Diversity wird allerdings der Versuch unternommen, diese Information nachträglich direkt aus den Beobachtungsdaten zu entnehmen. Die Methode geht zurück auf Arbeiten von Gonsalves \& Chidlaw (1979), und Gonsalves (1982). Alle wesentlichen Grundlagen und die Anwendung auf reale Daten werden bei Löfdahl (1996) beschrieben.

Wichtiges Ziel der vorliegenden Arbeit war es, zu untersuchen, ob diese Methode der Bildrekonstruktion geeignet ist für die Verarbeitung schmalbandiger, spektroskopischer Daten. Dabei sollten wie üblich die Informationen über die Wellenfronten (und damit über die OTFs) aus den Aufnahmen im Breitbandkanal (CCD 1 und CCD 1a) ermittelt und damit dann die schmalbandigen Aufnahmen (CCD 2) entfaltet werden.

\section{Prinzip der Methode}

Die Grundlage des Verfahrens sei hier anhand der geometrischen Optik erläutert: Eine klar definierte „Fokalebene“ eines abbildenden Systems existiert nur dann, wenn es keine Abbildungsfehler enthält, die senkrecht auf den einfallenden Lichtstrahlen stehenden Wellenfronten $\phi$ vor dem Eintritt in das Teleskop eben sind und sie im Teleskop zu exakten Gauß'schen Kugelwellen geformt werden. In realen Fällen erzeugen sowohl das atmosphärische Seeing als auch instrumentelle Fehler Abweichungen der Wellenfronten von der Ebene bzw. der Kugelform. Dies führt dazu, dass der Brennpunkt bzw. bei 

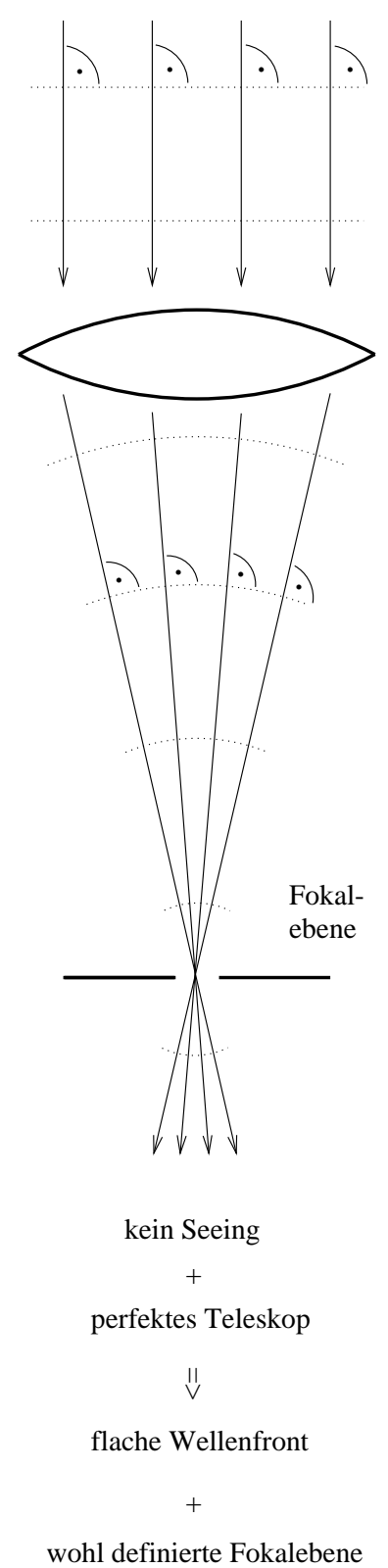
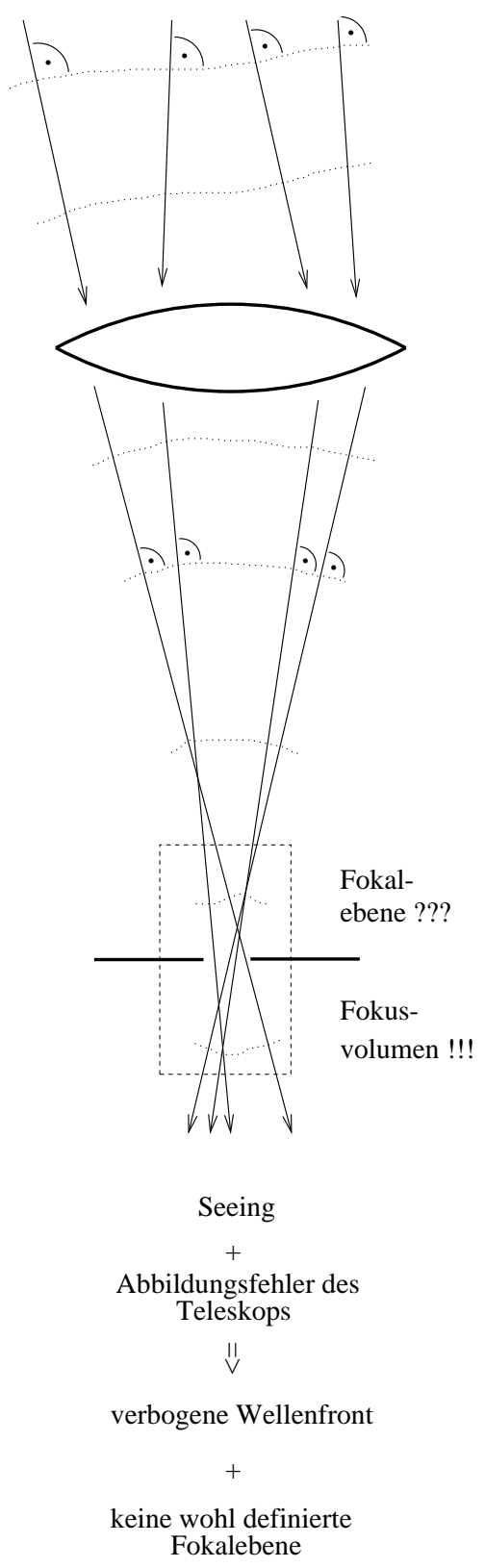

Abbildung 5.5: Funktionsprinzip der Phase Diversity Methode im Sinne der geometrischen Optik. Bei nicht parallel einfallenden Lichtstrahlen findet die Bildentstehung nicht in einer klar definierten Fokalebene statt, sondern in einem dreidimensionalen Volumen rund um den „mittleren“ Fokus.

flächigen Beobachtungsobjekten die Brennebene des Systems nicht mehr klar definiert ist; geometrisch konstruierte Lichtstrahlen aus verschiedenen Eintrittspunkten durch die Teleskop-Pupille kreuzen sich an unterschiedlichen Stellen, so dass nur eine „mittlere Brennebene“ angegeben werden kann. Abb. 5.5 soll dies verdeutlichen.

In einer wellenoptischen Betrachtungsweise ist natürlich auch bei einer fehlerfreien Ab- 


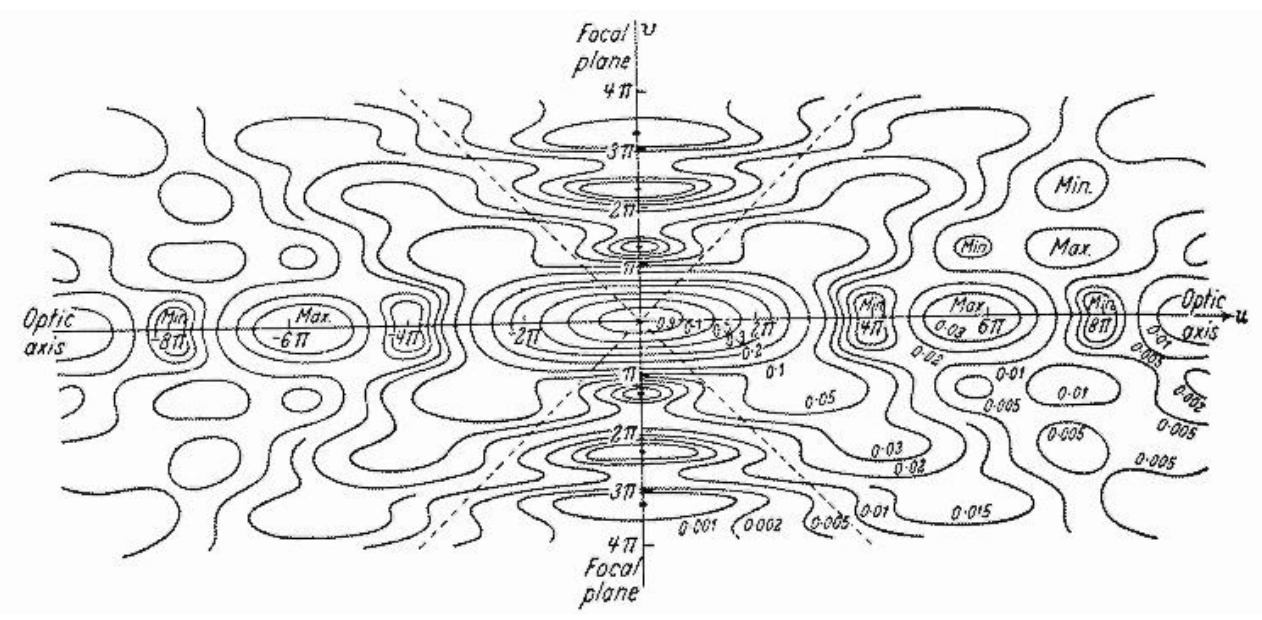

Abbildung 5.6: Intensitätsverteilung bei fehlerfreier Abbildung im Fokusvolumen. Aus Born \& Wolf (1971).

bildung von einem Fokusvolumen zu reden, innerhalb dessen sich die Entstehung der Abbildung vollzieht. Abb. 5.6 zeigt die Intensitätsverteilung in einem meridionalen Schnitt durch das Fokusvolumen. Dabei werde eine sich links befindende punktförmige Lichtquelle, deren Intensität im Fokus auf eins normiert ist, von einem perfekten optischen System mit kreisförmiger Eintrittspupille abgebildet. Die gestrichelten Linien bezeichnen dabei die Grenzen des Lichtbündels in der geometrischen Optik. Durch Rotation der Abbildung um die waagerecht liegende optische Achse des Systems erhält man in der Fokalebene das bekannte Airy-Scheibchen mit Beugungsringen. Abb. 5.7 zeigt als Beispiel Aufnahmen einer punktförmigen Lichtquelle, die mit einem optischen System abgebildet wird, das einen Astigmatismus aufweist. Beim Astigmatismus existieren zwei senkrecht zueinander stehende meridionale Schnitte durch das optische System, die verschiedene Brennweiten haben. Die linke Aufnahme wurde in der mittleren Brennebene, also zwischen diesen beiden Fokallinien gewonnen, die rechte genau in einer der beiden Fokallinien, also in der Brennebene des einen Schnitts. Eine erfahrene BeobachterIn könnte schon aus zwei solchen Aufnahmen qualitativ schließen, dass das Objekt eine punktförmige Lichtquelle sein muss, und dass als Abbildungsfehler ein Astigmatismus vorliegt.

Durch Untersuchung des Volumens rund um die mittlere Fokalebene kann also Information über die Form der Wellenfronten und über das beobachtete Objekt ermittelt werden. Dazu werden simultan zwei (oder mehr) Aufnahmen des Beobachtungsobjekts an verschiedenen Stellen des Fokusvolumens gemacht, eine in der mittleren Brennebene und eine in einer bestimmten Entfernung von dieser. Bei den vorliegenden Beobachtungen wurden hierfür die CCD-Kameras CCD 1 bzw. CCD 1a benutzt. Um beide Aufnahmen gleichzeitig zu erhalten, wurde der Lichtstrahl vorher durch einen Strahlteilerwürfel (SW 1a) aufgeteilt. Details hierzu lassen sich aus Kap. 3.2 und dort Abb. 3.2 und dem 

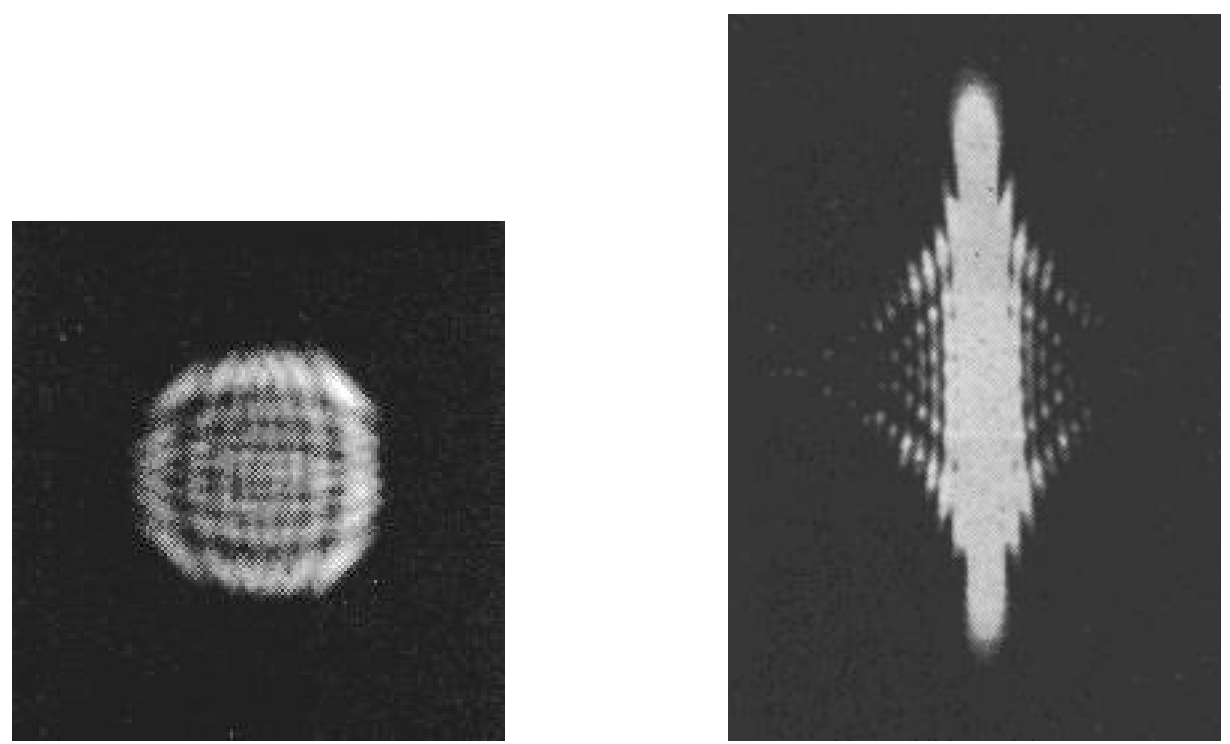

Abbildung 5.7: Abbildung einer punktförmigen Lichtquelle mit Astigmatismus. Links die Intensitätsverteilung in der mittleren Fokalebene, rechts in einer der Fokallinien. Aus Born \& Wolf (1971).

Text auf Seite 35 entnehmen.

Die unterschiedliche Fokusposition der beiden Aufnahmen von CCD 1 bzw. CCD 1a lässt sich auch als ortsabhängiger Phasenunterschied $\Delta \phi$ in der Eintrittspupille des Teleskops beschreiben. Die Fokusdifferenz der beiden Aufnahmen wurde bei den vorliegenden Beobachtungsdaten so gewählt, dass sie einer Phasendifferenz von einer Wellenlänge entspricht (Kap. 3.2.3). Die Methode beruht darauf, dass in einem System mit zwei unbekannten Größen, nämlich dem wahren Beobachtungsobjekt und der Form der Wellenfront, diese bestimmt werden können, wenn zwei Gleichungen in Form von zwei beobachteten Bildern zur Verfügung stehen. Die Phasendifferenz könnte prinzipiell auch anderer Natur sein als eine Fokusdifferenz, z. B. ein Astigmatismus, sie muss nur nach Art und Größe bekannt sein. Eine Fokusdifferenz lässt sich aber in der Praxis am leichtesten realisieren. Die Methode wird deshalb im internationalen Sprachgebrauch am häufigsten „Phase Diversity Methode“ genannt. In der Praxis sind die Gleichungssysteme allerdings aufgrund des in den Bildern vorhandenen Rauschens nicht exakt lösbar, es lassen sich daher auch mit dieser Bildrekonstruktionsmethode nur möglichst gute Schätzungen des wahren Objekts berechnen.

\section{Mathematische Grundlagen der Methode}

Die genaue mathematische Ableitung der Methode ist bei Löfdahl (1996) beschrieben, hier sollen deshalb nur die grundlegenden Gedanken dargestellt werden.

Zunächst liegen zwei an einem Zeitpunkt $t$ beobachtete Bilder vor, für die nach Glg. 2.17 


\subsection{Bildrekonstruktion}

im Fourierraum Folgendes gilt:

$$
I_{t, \mathrm{f}}=O \cdot S_{t, \mathrm{f}}+N_{t, \mathrm{f}} \quad \text { und } \quad I_{t, \mathrm{~d}}=O \cdot S_{t, \mathrm{~d}}+N_{t, \mathrm{~d}} .
$$

Dabei stehen die Indizes f bzw. d für die fokussierten bzw. defokussierten Größen. Im Folgenden sei desweiteren angenommen, dass die Rauschniveaus in beiden Aufnahmen gleich hoch sind. Diese Annahme ist gerechtfertigt, wenn mit gleichen (oder zumindest ähnlichen) Detektoren Bilder ähnlicher mittlerer Intensität aufgenommen werden.

Liegt die Form einer Wellenfrontaberration als reelle, zweidimensionale Funktion $\phi$ über dem Bereich der Eintrittspupille vor, so kann daraus zunächst die komplexe Pupillenfunktion $G$ gebildet werden:

$$
G(\phi)=\mathrm{e}^{\mathrm{i} \phi}=\cos \phi+\mathrm{i} \sin \phi .
$$

Außerhalb der Eintrittspupille ist die Pupillenfunktion null. Die OTF des optischen Systems ist nun gerade die Autokorrelationsfunktion (AKF) der Pupillenfunktion (Born \& Wolf (1971), dort Kap. 9.5.2):

$$
S(\phi)=\operatorname{AKF}(G(\phi)) .
$$

Somit stehen die OTFs in fokussiertem und defokussiertem Bild (Glg. 5.2) über die bekannte Phasendifferenz der Aufnahmen in einer bekannten, festen Beziehung:

$$
\phi_{t, \mathrm{~d}}=\phi_{t, \mathrm{f}}+\Delta \phi
$$

Die beiden Unbekannten des Gleichungssystems 5.2 sind das wahre Objekt $O$, und die OTF $S_{t, \mathrm{f}}$ für die fokussierte Aufnahme. Aus $S_{t, \mathrm{f}}$ ergibt sich mit Glgen. 5.3 - 5.5 sofort auch die OTF $S_{t, \mathrm{~d}}$ für die defokussierte Aufnahme. Die Lösung erfolgt durch ein iteratives Verfahren. Dabei wird zunächst eine bestimmte Form der Wellenfront angenommen und dann überprüft, ob diese Annahme anhand der beiden beobachteten Bilder gerechtfertigt ist. Dazu wird eine Fehlermetrik berechnet, die im Prinzip die beobachteten Bilder vergleicht mit dem, was hätte beobachtet werden müssen, wenn die angenommene Wellenfront korrekt gewesen wäre:

$$
L=\sum_{\vec{s}}\left\{\left|I_{t, \mathrm{f}}-\widehat{O} \cdot \widehat{S}_{t, \mathrm{f}}\right|^{2}+\left|I_{t, \mathrm{~d}}-\widehat{O} \cdot \widehat{S}_{t, \mathrm{~d}}\right|^{2}\right\}
$$

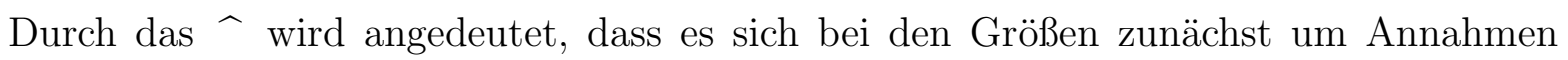
oder Schätzungen handelt. Eine Schätzung des Objekts ergibt sich vorläufig durch eine Entfaltung der beobachteten Bilder mit den zunächst angenommenen OTFs:

$$
\widehat{O}=\frac{I_{t, \mathrm{f}} \cdot \widehat{S}_{t, \mathrm{f}}^{*}+I_{t, \mathrm{~d}} \cdot \widehat{S}_{t, \mathrm{~d}}^{*}}{\left|\widehat{S}_{t, \mathrm{f}}\right|^{2}+\left|\widehat{S}_{t, \mathrm{~d}}\right|^{2}}
$$

Durch schrittweise Veränderung der angenommenen Wellenfront $\widehat{\phi}$ wird nun versucht, die Fehlermetrik $L$ zu minimieren. Man geht dann davon aus, dass man eine optimale 
Schätzung der Wellenfront gefunden hat, mit deren Hilfe sich ebenfalls eine bestmögliche Schätzung des wahren Objekts nach Glg. 5.7 errechnen lässt. Aufgrund des in den Beobachtungen enthaltenen Rauschens und evtl. nicht vollständiger Parametrisierung der Wellenfront kann aber die Fehlermetrik nicht den Wert null annehmen, folglich auch nie das wirklich wahre Objekt berechnet werden, sondern immer nur eine im Rahmen der Messwerte und des Rechenverfahrens bestmögliche Schätzung.

Zunächst muss aber eine geeignete mathematische Formulierung für Wellenfrontfehler $\phi$ gefunden werden. Hierfür bietet sich eine Zerlegung in Zernike-Polynome Noll (1976), Born \& Wolf (1971)] an. Diese bilden einen Satz von zweidimensionalen Basisfunktionen $\phi_{i}$, aus denen mit den entsprechenden Koeffizienten $c_{i}$ die Wellenfrontaberrationen als Funktionen des Ortsvektors $\vec{x}$ gebildet werden können:

$$
\phi(\vec{x})=\sum_{i} c_{i} \phi_{i}
$$

Dabei entsprechen die Polynome niedriger Ordnung den klassischen optischen Abbildungsfehlern: Verschiebung, Defokussierung, Astigmatismus, sphärische Aberration, Koma usw. In Abb. 5.8 sind die ersten 12 Zernike-Polynome grafisch dargestellt, die als geometrische Abweichungen der Wellenfront von der Ebene in der Eintrittspupille des Teleskops zu verstehen sind.

Für eine vollständige Darstellung der Wellenfront würde eine unendlich große Anzahl von Zernike-Polynomen benötigt. Die räumliche Strukturierung der Polynome wird mit zunehmender Ordnung immer feiner. Wenn man davon ausgeht, dass eine grobe Beschreibung der Wellenfrontfehler durch die grob strukturierten, niedrigeren Ordnungen der Zernike-Polynome eine untere Grenze für die anzubringenden Korrekturen darstellt, kann die Anzahl der Zernike-Polynome begrenzt werden. In der vorliegenden Arbeit wurde mit Zerlegungen in 8 bis 14 Zernike-Polynome experimentiert.

Darüber hinaus ist eine exakte Wiedergabe der Wellenfront auch nicht erforderlich, da nach dem Maréchal'schen Kriterium (Born \& Wolf (1971)] eine Abbildung als fehlerfrei betrachtet werden kann, wenn die mittlere quadratische Abweichung der Wellenfront von der idealen Form weniger als $\lambda / 14$ beträgt. Dieser Wert kann auch als Abbruchkriterium für den Iterationsprozess bei der Annäherung an die wahre Wellenfront verwendet werden.

Durch diese Darstellung der Wellenfront erhält man den oben beschriebenen Ausdruck für die Fehlermetrik (Glg. 5.6) als hochdimensionale Funktion, die von allen einzelnen Koeffizienten der Zerlegung, den „Zernike-Koeffizienten“, abhängt. Durch Anwendung des Newton-Verfahrens wird nun iterativ das Minimum der Fehlermetrik gesucht, bei dem eine bestmögliche Darstellung der Wellenfront und damit eine Abschätzung des wahren Objekts vorliegt.

Es muss hier noch darauf verwiesen werden, dass die Zernike-Polynome einen Satz von Orthonormalfunktionen auf dem Einheitskreis bilden. In der vorliegenden Arbeit wurde aber teilweise aufgrund der Teleskop-Geometrie (vgl. Kap. 3.1) mit nicht kreisförmigen 

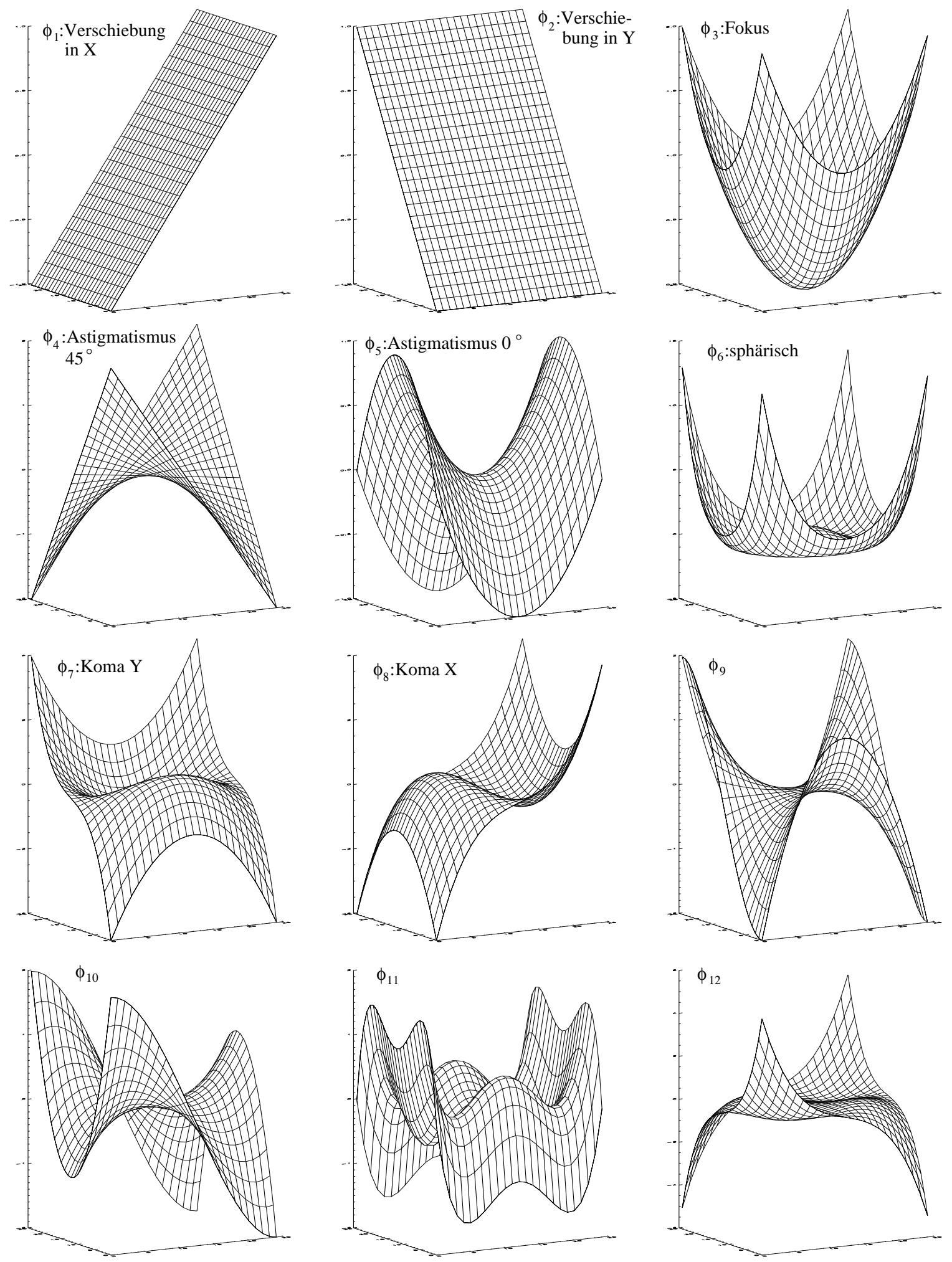

Abbildung 5.8: Die ersten 12 Zernike-Polynome als Funktion willkürlich gewählter Ortskoordinaten in der Eintrittspupille des optischen Systems. 
Eintrittspupillen beobachtet. Die tatsächliche Pupillenform wurde während der Beobachtungen fotografisch erfasst und bei der Berechnung der Wellenfronten berücksichtigt. Dadurch geht aber die Orthonormalität der Zernike-Polynome verloren. Das bedeutet, dass bei der Suche nach der Ableitung der Fehlermetrik nach den Zernike-Koeffizienten die Änderung eines Koeffizienten einen geringen Einfluss auf die anderen Koeffizienten hat. Da die Abweichungen von der Kreisform aber für die hier besprochenen Beobachtungsdaten gering sind, sollte das Newton-Verfahren trotzdem das Finden des Minimums der Fehlermetrik ermöglichen.

\section{Bestimmung der OTF und Rekonstruktion der wahren Objekte}

Bei der praktischen Durchführung der Berechnungen wurde auf ein Programm von Ac-

ton (1996) zurückgegriffen, welches eine Übersetzung des Programms von Löfdahl (1996) von der Programmiersprache ANA in die Programmiersprache IDL darstellt. Es wurde auf die speziellen Erfordernisse der vorliegenden Anwendung angepasst und inhaltlich überarbeitet. Bei der Verarbeitung umfangreicher Beobachtungsdaten, die sich u. a. in den beobachteten Objekten, vor allen Dingen aber in der Qualität des Seeings stark unterschieden, musste schnell festgestellt werden, dass der Algorithmus sehr unzuverlässig zu einer vernünftigen Abschätzung von Wellenfrontfehlern und Objekt konvergiert und sehr häufig nur unbefriedigende Ergebnisse liefert. Insbesondere zeigte sich, dass der Iterationsvorgang häufig zu lokalen Minima der Fehlermetrik konvergiert, bei denen die Zernike-Koeffizienten der gefundenen Zerlegung nicht die wahre, während der Aufnahme der Bilder vorliegende Wellenfront beschreiben. Abhängig ist dies u. a. vom Ausgangspunkt der Iteration, also davon, welche Wellenfront als erste Abschätzung angenommen wird (meistens eine ebene Wellenfront, also alle Zernike-Koeffizienten gleich null), oder von der Anzahl der für die Zerlegung verwendeten Zernike-Polynome. So kommen häufig Fälle vor, dass z. B. Zerlegungen in 8, 11 oder 14 Polynome schnell zu vernünftigen, beinahe identischen Ergebnissen führen, dagegen Zerlegungen in 10 oder 13 Polynome eine schlechte Konvergenz ergeben bei Zernike-Koeffizienten, die unrealistisch hoch sind und entsprechend eine schlechte Rekonstruktion des wahren Objekts liefern. Ebenfalls kommt es bei schlechterem Seeing, wenn also schon im fokussierten Bild weniger Strukturen erkennbar sind, leichter zu Fehlberechnungen des wahren Minimums der Fehlermetrik. Häufig kann nur einzeln qualitativ „per Hand“ entschieden werden, ob die Rechnung vernünftige, realistische Werte liefert.

In den Fällen, in denen der Algorithmus realistische, vertrauenswürdige Ergebnisse liefert, passen die berechneten Wellenfronten bzw. die dazugehörigen PSFs qualitativ gut zu den beobachteten Bildern, d. h. Teilbilder mit gutem Seeing, die viele Feinstrukturen zeigen, ergeben flache, wenig strukturierte Wellenfronten mit scharf begrenzten PSFs, während Bilder mit offensichtlich schlechtem Seeing zu stark strukturierten Wellenfronten mit breiten, unregelmäßig geformten PSFs führen. Auch treten häufig in den Bildern mit bloßem Auge sichtbare typische Abbildungsfehler wie einseitige Verzerrungen (Astig- 

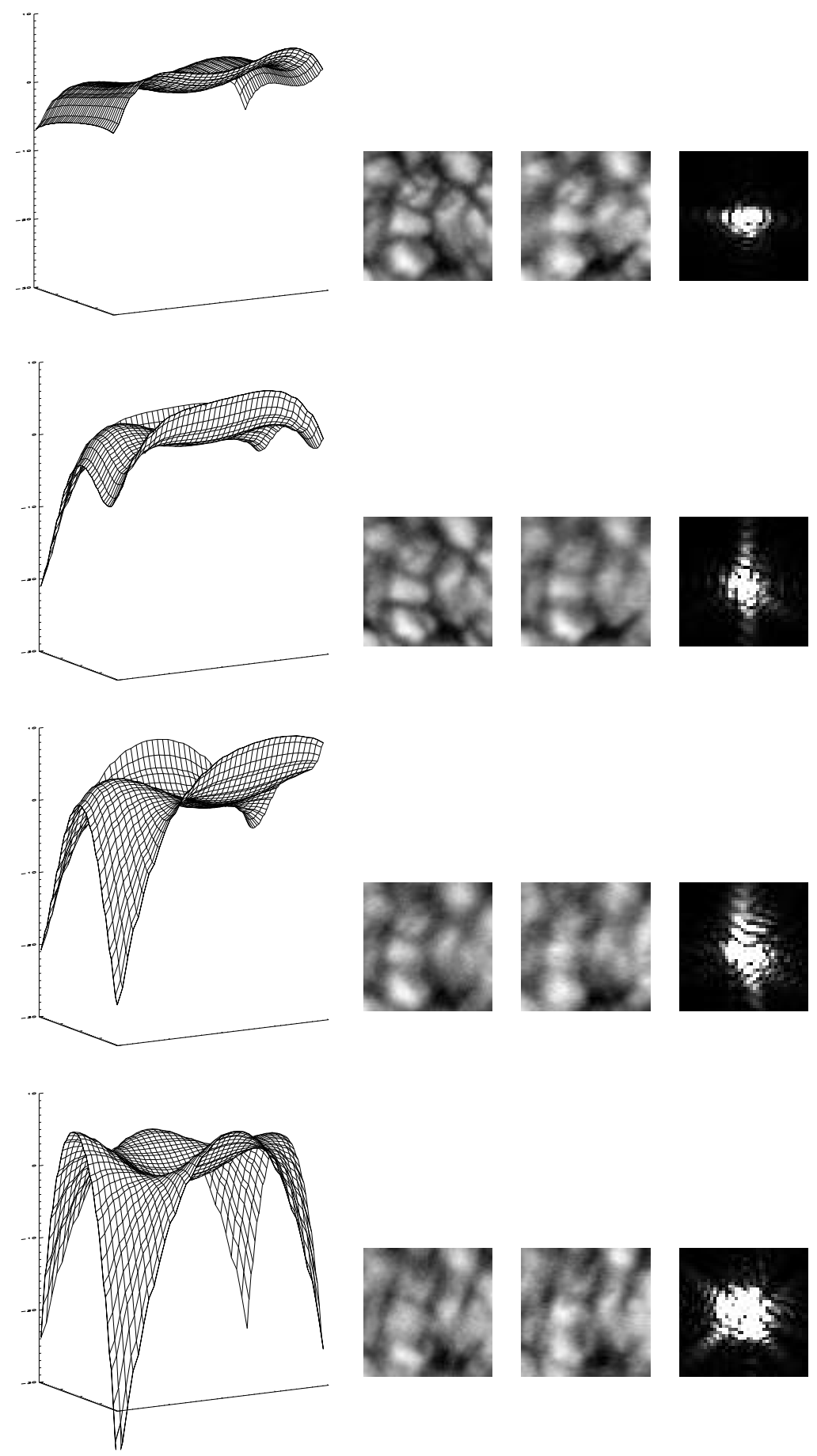

Abbildung 5.9: Mit der Phase Diversity Methode berechnete Wellenfronten zu den Bildpaaren 18, 8, 11 und 9 aus Scan s5_4 in willkürlichen Einheiten. Die zugehörigen Bildpaare aus fokussiertem bzw. defokussiertem Rohbild sind jeweils rechts davon zu sehen. Ganz rechts ein Ausschnitt der zu der Wellenfront gehörenden PSF, die Darstellung wurde so gewählt, dass die grobe Form der PSF sichtbar wird. 
matismen u. Ä.) auf, die sich ebenfalls in den berechneten Zernike-Koeffizienten und den PSFs wiederfinden. Abb. 5.9 zeigt für vier Bildpaare mit zunehmend schlechterem Seeing jeweils die mit dem Phase Diversity Algorithmus berechnete Wellenfront, das Bildpaar und die zugehörige PSF. Weitere mit dieser Methode berechnete PSFs finden sich bei Koschinsky et al. (1999).

Es ist prinzipiell möglich, mit einiger Handarbeit solange an den einzelnen Datenfeldern zu arbeiten, bis man für jedes eine gute, zuverlässige Abschätzung der Wellenfront und damit des wahren Objekts erhält. Allerdings muss man bedenken, dass schon jedes einzelne Bild in kleine Teilbilder von der Größe der isoplanaren Gebiete zerlegt wird, und dann noch Rekonstruktionen zu vielen (hier 28) spektralen Positionen nötig sind, die alle erfolgreich und mit etwa gleichmäßiger Qualität durchgeführt werden müssen, um am Ende einen spektroskopisch auswertbaren Datensatz zu erhalten.

Die Berechnung der Wellenfront aus den beiden breitbandigen Bildern von CCD 1 und CCD 1a ergibt als Nebenprodukt bereits eine Rekonstruktion des wahren, breitbandigen Objekts nach Glg. 5.7. Der Informationsgehalt über die kleinen räumlichen Strukturen kann aber gerade bei schlechtem Seeing gering sein und deshalb im Rauschen verloren gehen. Es muss deshalb auch hier noch das schon in Kap. 5.1.5 angesprochene und in Kap. 8 genauer beschriebene Rauschfilter angewendet werden. Häufig wird dieses dabei so eng bemessen sein, dass auch die Rekonstruktion wenig zusätzliche Feinstrukturen enthält. Andererseits kann es vorkommen, dass geringe, verbleibende Rauschanteile bei den kleinen Strukturen oder geringe Fehlberechnungen der Wellenfront und damit der zur Verstärkung der Strukturen herangezogenen OTF zur Entstehung von Fehlstellen („Artefakten") in den Rekonstruktionen führen, die meistens leicht an ihrer streifenförmigen Struktur zu erkennen sind. Ebenfalls ergeben sich starke Streifenmuster in den Rekonstruktionen, wenn die Berechnung der Wellenfrontaberration in einem falschen, lokalen Minimum der Fehlermetrik konvergiert ist.

Generell lässt sich feststellen, dass die Berechnung der Wellenfront am ehesten gelingt und sich damit dann eine gute Rekonstruktion ergibt, wenn das Seeing in den Einzelbildern außergewöhnlich gut ist. Bei etwas schlechterem Seeing kommt es schnell zu Fehlberechnungen der Wellenfront und zu streifenförmigen Mustern in den Rekonstruktionen oder einfach zu solchen Rekonstruktionen, die nicht mehr Feinstrukturen zeigen als die Rohbilder.

Eine gewisse Verbesserung der Rekonstruktionsergebnisse kann erreicht werden, indem die Informationen aus allen an einer bestimmten spektralen Position aufgenommenen Bildern für eine gemeinsame Rekonstruktion genutzt werden. Dazu werden nach der Berechnung der Wellenfronten alle einzelnen Bilder zusammen mit ihren Wellenfrontfehlern zu einer einzigen Rekonstruktion verarbeitet:

$$
\widehat{O}=H \cdot \frac{\sum_{t}\left(I_{t} \cdot \widehat{S}_{t}^{*}\right)}{\sum_{t}\left|\widehat{S}_{t}\right|^{2}} .
$$

Hier können im Breitbandkanal sowohl die fokussierten wie auch die defokussierten Bil- 
der mit ihren zugehörigen Wellenfronten bzw. OTFs herangezogen werden, praktisch bringen aber die defokussierten Bilder in einem solchen Ensemble mehrerer Bildpaare keinen wesentlichen Zugewinn an Bildinformationen, so dass sie aus Gründen der Rechenkapazität hier auch weggelassen werden können. Es werden also nur die fokussierten Bilder für die Rekonstruktion des breitbandigen Objekts verwendet.

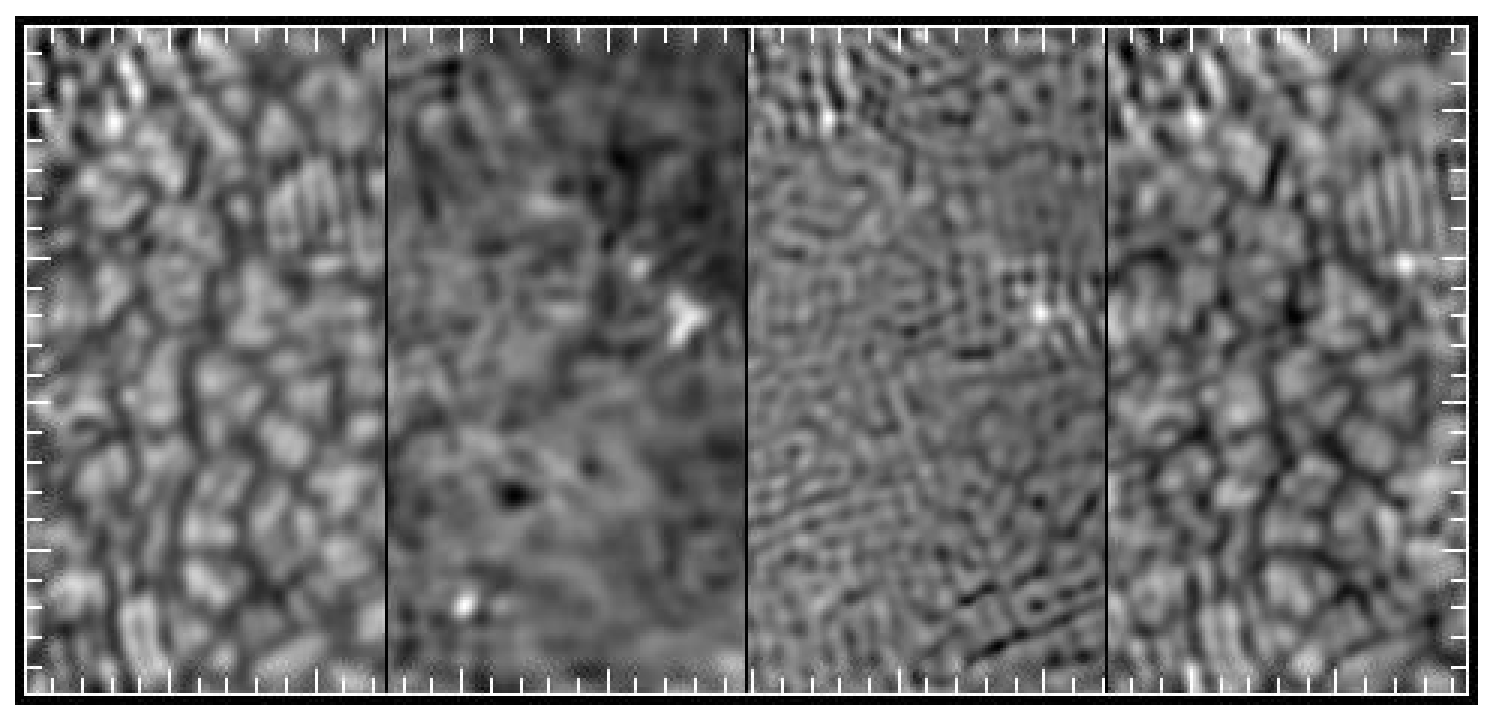

Abbildung 5.10: Ergebnisse der Rekonstruktion mit der Phase Diversity Methode bei Scan s5_4. Von links nach rechts CCD 2-Bilder aus dem Kontinuum und dem Linienkern, die Magnetfeldkarte und das Geschwindigkeitsbild. Der Abstand der Strichmarken entspricht 1".

Glg. 5.9 kann aber ebensogut auch für die Rekonstruktion des schmalbandigen Objekts benutzt werden. Hierzu werden alle CCD 2-Bilder einer spektralen Position verwendet, zusammen mit den im Breitbandkanal berechneten OTFs der fokussierten CCD 1-Bilder. Abb. 5.10 zeigt für den Scan s5_4 zwei rekonstruierte Bilder im Kontinuum und im Linienkern. Im Kontinuumsbild sind oben rechts streifenförmige Artefakte einer nicht korrekten Rekonstruktion zu erkennen. Die hier nicht gezeigten Rekonstruktionen an den 26 anderen spektralen Positionen zeigen meist ähnliche Streifenmuster, allerdings an unterschiedlichen Stellen des Bildfelds. Diese finden in der anschließenden, in Kap. 5.2 näher beschriebenen Bestimmung der Magnetfelder und Geschwindigkeiten ihren Niederschlag. Die auf diese Weise erstellten Magnetfeldkarten sind deshalb kaum zu gebrauchen, weil bei den sichtbaren Strukturen häufig nicht klar ist, ob sie einer echten Struktur auf der Sonnenoberfläche entsprechen, oder ob sie durch Artefakte und Fehlrekonstruktionen an einer oder mehreren spektralen Positionen entstehen.

Für die letztendliche Auswertung der vorliegenden Beobachtungsdaten wurde deshalb eine andere, zuverlässigere Bildrekonstruktionsmethode gewählt, die im Folgenden beschrieben werden soll. 


\subsubsection{Speckle-Interferometrie}

\section{Rekonstruktion der CCD 1-Bilder}

Mit der Speckle-Interferometrie steht eine etablierte, gut entwickelte und zuverlässige Methode der Bildrekonstruktion zur Verfügung. Sie nahm ihre Anfänge mit Arbeiten von Labeyrie (1970), in denen die korrekten Autokorrelationsfunktionen von Objekten der Stellarastronomie aus vielen einzelnen kurzbelichteten Aufnahmen berechnet wurden. Diese Autokorrelationsfunktionen geben jedoch nur für symmetrische Objekte oder Objekte wie Doppelsterne unmittelbare Auskunft über die wahre, im Einzel- oder Langzeitbild nicht aufgelöste Struktur dieser Objekte. Anwendung auf flächenhafte Objekte fand die Methode durch fortführende Arbeiten von Knox \& Thompson (1974) und Weigelt (1977). Eine umfassende Darstellung der Methode findet sich z. B. bei de Boer (1993), dort finden sich auch alle wichtigen Literaturhinweise. Hier soll nur in groben Zügen die Wirkungsweise der Methode angerissen und an einem Beispiel erläutert werden.]

Grundlage auch dieses Verfahrens ist die Tatsache, dass in kurzbelichteten Aufnahmen die momentane OTF, bzw. deren Betrag, meistens bis hin zur Grenzfrequenz des Teleskops $s_{\text {grenz }}$ (Glg. 2.18) größer als null ist. Deshalb sind in solchen Aufnahmen Informationen über diese kleinen Strukturen enthalten. Karo \& Schneidermann (1978) haben bei Messungen an Sternen festgestellt, dass die beobachteten Powerspektren im Bereich großer Raumfrequenzen nur wenig geringere Werte annehmen, wenn die Belichtungszeiten von $5 \mathrm{~ms}$ auf $40 \mathrm{~ms}$ verlängert werden. Erst bei längeren Zeiten als $80 \mathrm{~ms}$ messen sie einen deutlichen Verlust kleiner Strukturen, wie in einer langbelichteten Aufnahme. Es kann also damit gerechnet werden, dass Bildrekonstruktionsmaßnahmen dieser Art bis zu Belichtungszeiten von 40-60 ms gute Resultate bringen. Allerdings ist zu beachten, dass diese Werte je nach den äußeren Gegebenheiten stark variieren können, insbesondere sich am Tage anders verhalten können als bei Nacht.

Wenn das Powerspektrum der mittleren momentanen OTF, das „Speckle Transfer Funktion (STF)“ genannt wird, bekannt ist, können die wahren Powerspektren des beobachteten Objekts aus den gemessenen Powerspektren und der STF berechnet werden. Bei stellarastronomischen Beobachtungen lässt sich häufig die STF direkt aus den Powerspektren eines als punktförmig angenommenen Sterns im Beobachtungsgebiet entnehmen. Dies ist bei flächenhaften Objekten wie der Sonnenoberfläche, auf der kein als bekannt vorauszusetzendes Testobjekt existiert, nicht möglich. Basierend auf der Arbeit von v. d. Lühe (1984) besteht aber die Möglichkeit, aus dem Vergleich der beobachteten Powerspektren von Kurzzeitaufnahmen eines Objekts mit dem Powerspektrum eines gemittelten Bilds dieses Objekts die Information über das (zunächst ja unbekannte) Objekt zu eliminieren und statt dessen den Fried-Parameter (Fried (1966)] abzuschätzen, der die

\footnotetext{
${ }^{1}$ Der Begriff „Speckle-Rekonstruktion“ wird sowohl für die Methode, als auch für deren Durchführung und insbesondere auch für das Resultat, das speckle-rekonstruierte Bild, verwendet.
} 


\subsection{Bildrekonstruktion}

Qualität des Seeings beschreibt. Diese Methode wird „Methode des spektralen Quotienten" genannt. Mit der Kenntnis des Fried-Parameters lässt sich nach einem Modell von Korff (1973) die Form der STF abschätzen, mit der die Rekonstruktion der Amplituden der Fourierdarstellung des beobachteten Objekts vorgenommen werden kann. Hierbei sei noch erwähnt, dass die zur Korrektur verwendete STF gleichzeitig die atmosphärischen Seeingeinflüsse enthält wie auch die Übertragungsfunktion des Teleskops.

Eine vollständige Beschreibung der Fouriertransformierten der Intensitätsverteilung des wahren Objekts ist durch

$$
O(\vec{s})=|O(\vec{s})| \cdot \exp [i \phi(\vec{s})]
$$

gegeben. Allerdings gehen bei der Bildung der Powerspektren durch die Berechnung der Betragsquadrate die Phasenbeziehungen verloren, so dass durch Rücktransformation in den Bildraum kein echtes Bild des Objekts erhalten werden kann. Die Phasen in Glg. 5.10 müssen in einem getrennten Verfahren berechnet werden, um eine Zuordnung von rekonstruierten Fourier-Amplituden zu konkreten Orten im Bild zu ermöglichen. Das Problem ist hier, dass der Mittelwert der Phasen zu einer bestimmten Raumfrequenz $s$ nur dann aussagekräftig ist, wenn $s$ kleiner als die Seeing-Grenzfrequenz ist, das ist die Frequenz, ab der in langbelichteten Bildern keine Strukturen mehr erkennbar sind. Für größere Frequenzen sind die Fourierphasen statistisch verteilt, der Mittelwert eines hinreichend großen Ensembles von Aufnahmen wird also null ergeben. Entscheidend ist aber, dass der Mittelwert von Phasendifferenzen in kurzbelichteten Aufnahmen sehr wohl aussagekräftig ist. Grob gesagt, wenn die Phase bei einer gewissen Raumfrequenz $s$ bekannt ist, lässt sich auch die Phase einer Nachbarfrequenz $s+\Delta s$ ermitteln. Unter der Annahme, dass bei $\vec{s}=0$ auch die Phase null ist und die Phase der dem Nullpunkt am nächsten liegenden Raumfrequenz durch Seeing nicht beeinflusst worden ist und deshalb durch einfache Mittelung bestimmt werden kann, kann nun auf rekursive Weise jede weitere Objektphase ermittelt werden. Das verglichen mit der Knox-Thompson-Methode ( Knox \& Thompson (1974) zuverlässigere „Speckle Masking“ Verfahren ermittelt die Informationen mehrfach auf redundante Weise, indem die Phasen für bestimmte Raumfrequenzen auf verschiedenen Wegen mit verschiedenen Phasendifferenzen zu bekannten, vorher bestimmten Raumfrequenzen ermittelt werden (Weigelt (1977)]. Zusammen mit den zugehörigen Fourier-Beträgen, die man aus der Labeyrie-Methode erhalten hat, ergibt sich eine komplette Beschreibung der Fouriertransformierten der Objektintensitätsverteilung und nach Rücktransformation in den Ortsraum das wahre, rekonstruierte Bild des beobachteten Objekts.

In der vorliegenden Arbeit wurde ein vorhandenes Softwarepaket auf den speziellen Bedarf angepasst und verwendet. Es wurde von C. R. de Boer im Rahmen seiner Dissertation geschrieben (de Boer (1993)) und von P. Sütterlin weiterentwickelt. Abb. 5.11 zeigt exemplarisch den großen Erfolg der Speckle-Interferometrie. Bearbeitet wurden die 140 CCD 1-Einzelbilder des Scans s5_4 vom 22.10.99. Gezeigt sind das beste Einzelbild des Scans, das Langzeitbild, das durch Mittelung aller Einzelbilder erzeugt wurde, so- 


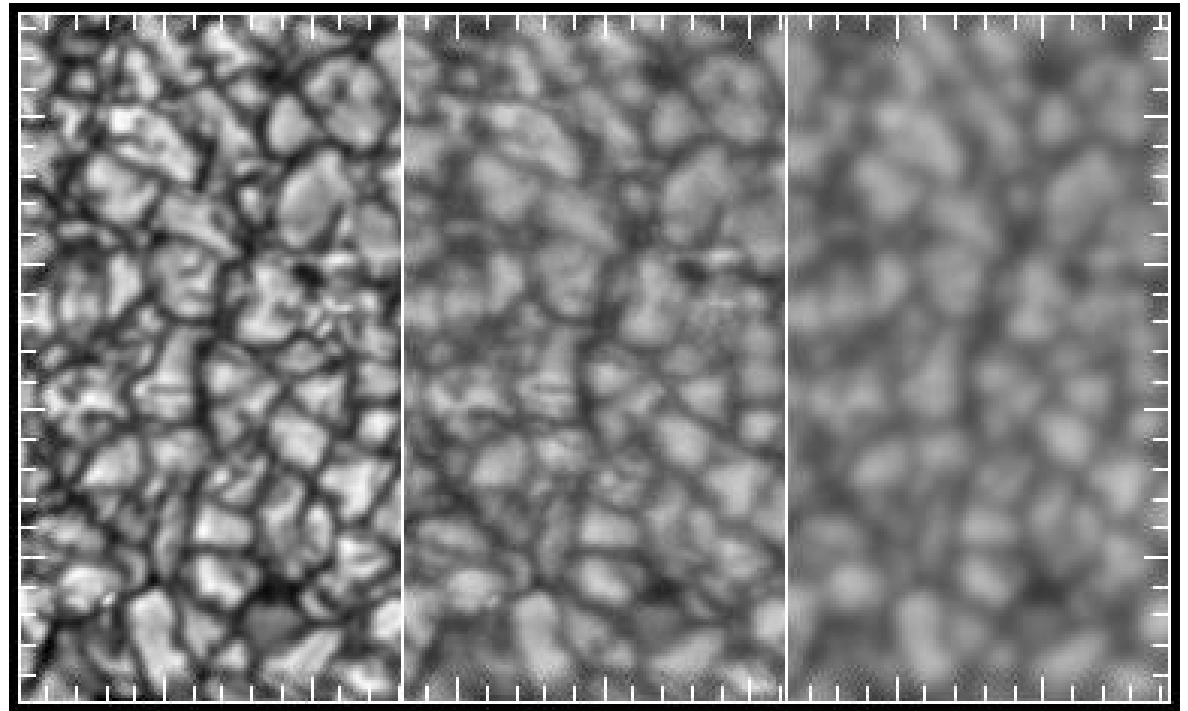

Abbildung 5.11: Speckle-Rekonstruktion der CCD 1-Bilder des Scans s5_4 (links), bestes Einzelbild des Scans (Mitte) und aus allen 140 Einzelbildern gemitteltes Langzeitbild (rechts). Der Abstand der Strichmarken entspricht $1^{\prime \prime}$.

wie die Speckle-Rekonstruktion. Man beachte den Zugewinn des rekonstruierten Bilds an Kontrast und Detailreichtum verglichen mit dem besten Einzelbild. Weitere SpeckleRekonstruktionen sind in Abb. 2.1 und Abb. 6.3 gezeigt.

\section{Entfaltung der CCD 2-Bilder}

Für die schmalbandigen Daten kann die Speckle-Interferometrie nicht angewendet werden, da sie nur dann gute Ergebnisse liefert, wenn hinreichend viele Einzelbilder in die Rekonstruktion einbezogen werden können. Erfahrungsgemäß ist dies bei Aufnahmen von der Sonnenoberfläche ab ca. 50-100 Bildern der Fall. Hier stehen aber an jeder Wellenlägenposition nur je fünf Bilder zur Verfügung. Schon die Bestimmung des Fried-Parameters birgt dann Schwierigkeiten, weil sich aus fünf Einzelaufnahmen kein repräsentativer Wert für die Langzeit-OTF berechnen lässt. Auch die Mittelung der Phasendifferenzen bei der Speckle-Masking-Methode ergäbe nur unzuverlässige Werte.

Hier kann man aber die Informationen aus dem Breitbandkanal benutzen, um quantitative Aussagen über die momentane OTF während jeder einzelnen Aufnahme zu machen. Da die CCD 1- und CCD 2-Bilder streng simultan aufgenommen wurden, kann davon ausgegangen werden, dass die OTFs, die für die CCD 1-Bilder bestimmt werden, auch für die CCD 2-Bilder Gültigkeit haben. Bezugnehmend auf Glg. 2.17 gilt damit

$$
I_{\mathrm{b}, t}=O_{\mathrm{b}} \cdot S_{t}+N_{\mathrm{b}, t} \quad \text { und } \quad I_{\mathrm{s}, t}=O_{\mathrm{s}} \cdot S_{t}+N_{\mathrm{s}, t}
$$

mit den Indizes b für breitbandig und s für schmalbandig und $t$ für jeden Zeitpunkt, an dem ein Bildpaar aufgenommen wurde. Lediglich Einflüsse der optischen Komponenten 


\subsection{Bildrekonstruktion}

ab dem Strahlteilerwürfel SW 1 (Abb. 3.2) könnten leichte Unterschiede der OTFs für die beiden Kameras erzeugen. Diese werden aber im Rahmen der Messungenauigkeit als vernachlässigbar betrachtet. Dieses Vorgehen ist insofern unkritisch, als dass für den Fall von auftretenden Abbildungsfehlern diese mit hoher Wahrscheinlichkeit für die CCD 2 gelten würden, da für diese ungleich mehr optische Komponenten im Strahlengang eingebaut sind. Deshalb würden Korrekturen, die mit der bei CCD 1 bestimmten OTF durchgeführt werden, zu einer Unterkorrektur führen. Dies ist besonders angesichts des die Auflösung ohnehin begrenzenden Rauschens unkritischer als eine Überkorrektur, welche falsche Strukturen in den Rekonstruktionen erzeugen könnte.

Die verwendete Rekonstruktionsmethode wurde erstmalig von Keller \& v. d. Lühe (1992) vorgestellt. Ausgiebigen Gebrauch der Methode machten auch Krieg (1999a), Krieg et a . (1999 b), Krieg et al. (2000), Hirzberger et al. (2001), Koschinsky et al. (2001) und Ritter (2001), die ebenfalls Daten verwendeten, die mit der in Kap. 3 beschriebenen Beobachtungsanlage aufgenommen wurden.

In Glg. 5.11 ist in der Beziehung für den Breitbandkanal neben dem Bild $I_{\mathrm{b}}$ auch das wahre Objekt $O_{\mathrm{b}}$ bekannt, wenn man davon ausgeht, dass die Speckle-Rekonstruktion das wahre Objekt hinreichend gut beschreibt und das Rauschen im Breitbandkanal $N_{\mathrm{b}}$ für einen Moment vernachlässigt wird. Diese Annahme ist gerechtfertigt, weil im Breitbandkanal aufgrund der wesentlich höheren Intensität das Rauschen etwa eine Größenordnung niedriger ist als im Schmalbandkanal. Für rauschfreie Bilder gilt dann exakt

$$
S_{t}=\frac{I_{\mathrm{b}, t}}{O_{\mathrm{b}}}
$$

Für den Fall, dass auch die CCD 2-Bilder rauschfrei sind, kann diese OTF nun im Prinzip in die Beziehung für die Schmalbandbilder in Glg. 5.11 eingesetzt werden, wodurch sich das Schmalbandobjekt zu

$$
O_{\mathrm{s}}=\frac{I_{\mathrm{s}, t}}{S_{t}}=\frac{I_{\mathrm{s}, t}}{I_{\mathrm{b}, t}} \cdot O_{\mathrm{b}}
$$

ergibt. In der Praxis funktioniert diese Entfaltung aber nicht, da der Nenner in Einzelfällen für beliebige Frequenzen verschwinden kann. Dies geschieht dort, wo die OTF Nullstellen aufweist oder das immer vorhandene Rauschen bei gleichzeitig kleiner OTF den Nenner noch kleiner werden lässt. Diese beiden Gründe führen dazu, dass der Ausdruck $I_{\mathrm{s}, t} / I_{\mathrm{b}, t}$ nicht exakt bestimmbar ist bzw. bei bestimmten Raumfrequenzen nicht definiert ist.

Mit Hilfe von Rauschfiltern können im Prinzip die Frequenzbereiche, in denen das Signal vom Rauschen überdeckt oder stark verfälscht wird, abgeschnitten werden. Aus den verbleibenden Informationen lassen sich dann OTFs bilden, deren grobe Struktur richtig ist, deren Feinstruktur aber oberhalb der abgeschnittenen Frequenzen verloren gegangen ist. Damit ist eine Entfaltung der Schmalbandbilder im Prinzip nach Glg. 5.13 möglich, allerdings ist der rekonstruierte Frequenzbereich entsprechend dem Informationsgehalt dieses einen Bildpaars zur Zeit $t$ gering. 
Ein besserer Ansatz beleuchtet die Frage, ob sich aus mehreren Bildpaaren gemeinsam ausreichende Information über den Quotienten $I_{\mathrm{s}, t} / I_{\mathrm{b}, t}$ sammeln lässt. Insbesondere stellt sich die Frage, ob sich verwertbare Aussagen über den Erwartungswert $\left\langle I_{\mathrm{s}} / I_{\mathrm{b}}\right\rangle$ dieser Größe machen lassen. Eine einfache, arithmetische Mittelung über fünf Bildpaare würde hier kein zuverlässiges Ergebnis liefern, da ein einzelnes, ungünstiges Rauschereignis in einem der Bildpaare den Nenner sehr klein machen und damit den ganzen Summanden unverhältnismässig groß machen könnte, so dass das arithmetische Mittel völlig verfälscht wäre (vgl. Krieg (1999a), S. 24). Dagegen kann ein gewichtetes Mittel ein wesentlich besseres Ergebnis liefern. Als Gewichtungsfunktion bietet sich das Powerspektrum des beobachteten Bilds $\left|I_{\mathrm{b}, t}\right|^{2}$ selbst an, da es immer dann kleine Werte annimmt, wenn das Signal-zu-Rausch-Verhältnis (SNR) klein ist. Mit anderen Worten: Immer wenn ein zu geringes Signal in $I_{\mathrm{b}, t}$ den Quotienten in Gefahr bringt, völlig falsche Werte anzunehmen, wirft es sich durch die Gewichtung selbst aus dem Rennen. Der Einfluss der Gewichtungsfunktion auf den Erwartungswert muss natürlich im Mittel wieder rückgängig gemacht werden, indem der Ausdruck durch die mittlere Gewichtungsfunktion dividiert wird. Bedeute das ^ eine Schätzung, so ist die mathematische Formulierung also

$$
\left\langle\frac{\widehat{I_{\mathrm{s}}}}{I_{\mathrm{b}}}\right\rangle=\frac{\sum_{t}\left(\left(I_{\mathrm{s}, t} / I_{\mathrm{b}, t}\right)\left|I_{\mathrm{b}, t}\right|^{2}\right)}{\sum_{t}\left|I_{\mathrm{b}, t}\right|^{2}}=\frac{\sum_{t}\left(I_{\mathrm{s}, t} I_{\mathrm{b}, t}^{*}\right)}{\sum_{t}\left|I_{\mathrm{b}, t}\right|^{2}}
$$

und liefert für all die Raumfrequenzen eine zuverlässige Abschätzung des Erwartungswerts, an denen in mindestens einem Bildpaar der Summation ein hinreichendes SNR vorhanden ist. Außerdem sei erwähnt, dass das Powerspektrum des Objekts $\left|O_{\mathrm{b}}\right|^{2}$ selbst bei allen Frequenzen größer als null sein muss. Dies ist aber für alle mit heutigen Teleskopen beobachteten Objekte der Sonnenoberfläche der Fall.

Mit dieser Schätzung des Quotienten $\left\langle I_{\mathrm{s}} / I_{\mathrm{b}}\right\rangle$ lässt sich nun leicht eine Schätzung des schmalbandigen Objekts in Glg. 5.13 berechnen:

$$
\widehat{O}_{\mathrm{s}}=H \cdot \frac{\sum_{t}\left(I_{\mathrm{s}, t} I_{\mathrm{b}, t}^{*}\right)}{\sum_{t}\left|I_{\mathrm{b}, t}\right|^{2}} \cdot O_{\mathrm{b}}
$$

Dabei ist H ein Rauschfilter, welches im Folgenden beschrieben werden soll. Ein Beispiel einer auf diese Weise berechneten Rekonstruktion ist u. a. in Abb. 5.13zu sehen, weitere Beispiele finden sich im Ergebniskapitel 6.

\section{Das Rauschfilter $\mathbf{H}$}

Wie schon in der Ableitung von Glg. 5.14 angedeutet, muss das Rauschfilter für all die Raumfrequenzen wirksam werden, bei denen der Erwartungswert $\left\langle I_{\mathrm{s}, t} / I_{\mathrm{b}, t}\right\rangle$ wegen mangelnden SNRs in allen Bildpaaren nicht zuverlässig bestimmt werden konnte. Unter der hier zulässigen Annahme, dass das Breitbandrauschen $N_{\mathrm{b}}$ gegenüber dem Schmalbandrauschen $N_{\mathrm{s}}$ vernachlässigt werden kann, lässt sich das Filter durch folgenden Ausdruck 


\subsection{Bildrekonstruktion}

erzeugen:

$$
H=1-\frac{\left\langle\left|N_{\mathrm{s}}\right|^{2}\right\rangle}{\left\langle\left|O_{\mathrm{s}}\right|^{2} \cdot|S|^{2}\right\rangle} .
$$

Der Erwartungswert des Rauschens $\left\langle\left|N_{\mathrm{s}}\right|^{2}\right\rangle$ kann recht leicht durch Mittelung der Powerspektren vieler einzelner Flatfieldaufnahmen berechnet werden, die zuvor durch die Verstärkungsmatrix dividiert und dadurch von den in Kap. 5.1.1 beschriebenen Einflüssen befreit wurden und nur noch den Rauschanteil enthalten. Eine Schätzung des Erwartungswerts im Nenner von Glg. 5.16 ist etwas schwieriger. Sie wurde in dieser Arbeit berechnet, indem die beobachteten Bilder $I_{\mathrm{s}, t}$ mit einem gleitenden Mittel über $3 \times 3$ Pixel geglättet und das mittlere Rauschniveau abgezogen wurde.

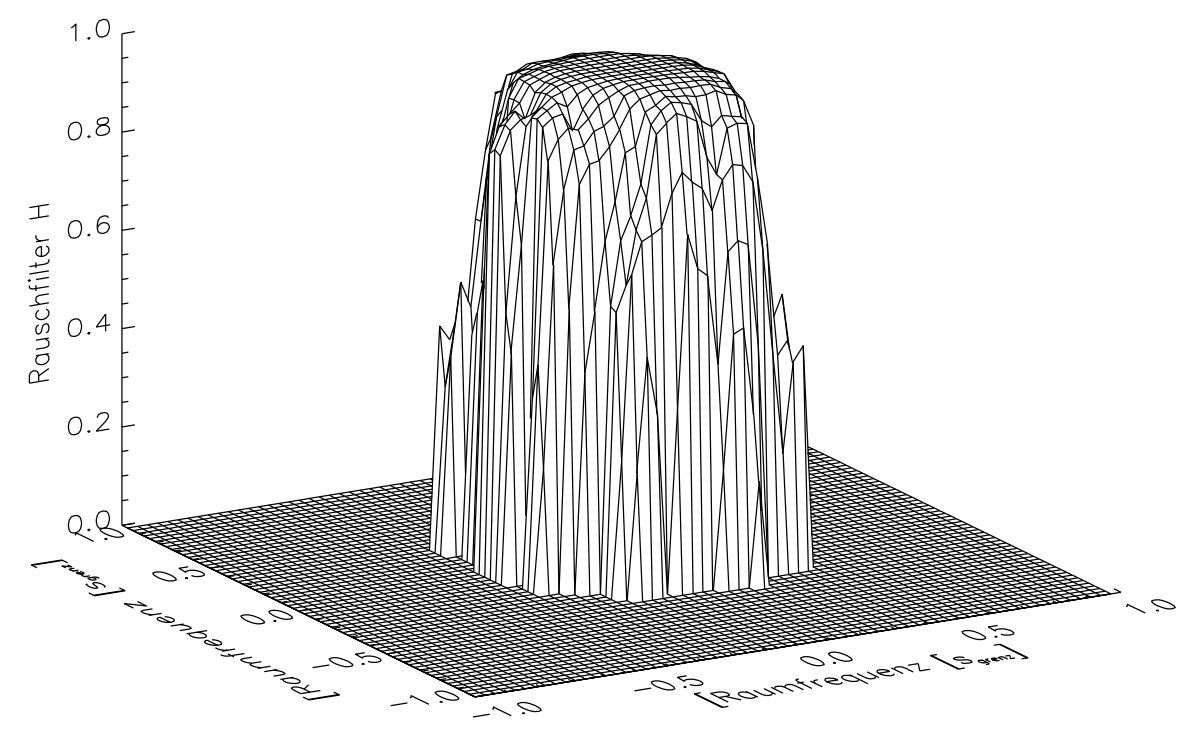

Abbildung 5.12: Beispiel eines Rauschfilters $H$, wie es bei Bildrekonstruktionen verwendet wird.

Damit geht das Filter immer dann, wenn in mindestens einem Bild nach Abzug des mittleren Rauschniveaus noch deutliches Signal übrig bleibt, gegen eins. Ist das Rauschen dagegen größer als das Signal in den Bildern, so nimmt Glg. 5.16 negative Werte an und wird auf null gesetzt. In der Praxis wird dies bereits dann gemacht, wenn der Wert des Filters kleiner als 0,2 wird. Es ist dann außerdem noch nötig, verbleibende Spitzen des Filters bei einzelnen Raumfrequenzen herauszunehmen. Abb. 5.12 zeigt ein typisches Rauschfilter.

Die genaue Herleitung dieses Filters ist bei Löfdahl (1996) beschrieben, ähnliche Herleitungen finden sich z. B. bei Brault \& White (1971) oder Keller \& v. d. Lühe (1992). Das hier beschriebene Filter stellt sich also immer auf die aktuellen Beobachtungsbe- 
dingungen ein, so dass die Schätzung des Objekts dem wahren Objekt im Rahmen der verfügbaren Informationen so nahe wie möglich kommt. Filter dieser Art werden deshalb auch „Optimumfilter" genannt.

Praktische Versuchsreihen haben gezeigt, dass die Filter fast immer eine recht regelmäßige Form haben. Über weite Frequenzbereiche sind sie fast eins, und fallen dann auf einem beinahe kreisrunden Gebiet in der zweidimensionalen Frequenzebene sehr steil auf null ab. Es erschien daher naheliegend, Versuche mit eher willkürlich erzeugten, kreisförmigen Filtern durchzuführen. Diese sind bei kleinen Frequenzen eins und fallen bei einer wählbaren Frequenz schnell auf null ab. Durch Ausprobieren verschiedener Filterbreiten und optische Kontrolle der Rekonstruktionen nach Glg. 5.15 bekommt man sehr schnell ein Gefühl dafür, welches räumliche Auflösungsvermögen in einem Scan erreicht werden kann, ehe deutlich „unechte“ Strukturen durch die fälschliche Verstärkung von Rauschen erzeugt werden. Bei geeigneter Wahl der Filterbreite ist die Qualität der Rekonstruktionen ebenso hoch wie bei solchen, in denen ein oben beschriebenes Optimumfilter verwendet wurde. Die Wahl der Art des Filters ist also von eher untergeordneter Bedeutung.

Der Vorteil kreisrunder, für alle Bilder gleicher Filter ist, dass man z. B. bei späteren Analysen von Powerspektren genau sagen kann, in welchen Frequenzbereichen das Power vom Filter abgeschnitten wurde, und dass dieses dann für alle Bilder gleichermaßen gilt. Auch bei der Berechnung von Differenzsignalen muss sichergestellt sein, dass beide Bilder mit demselben Filter bearbeitet wurden.

\subsubsection{Nachbearbeitung der Daten}

Nachdem für jede Wellenlängenposition eines Scans zwei Bilder rekonstruiert wurden, je eines für die beiden polarimetrischen Kanäle $\frac{1}{2}(I+V)$ und $\frac{1}{2}(I-V)$, muss für diesen Datensatz abschließend der allen rekonstruierten Bildern gemeinsame, größtmögliche Ausschnitt bestimmt und ausgeschnitten werden. Da die Seeingbedingungen von Bild zu Bild schwanken und schon das Ausschneiden der Teilbildserien aus den Rohbildern (Kap. 5.1.4) geringe Ungenauigkeiten aufweist, können sich die Bildfelder der rekonstruierten Bilder in der Lage wie auch in der Größe um wenige Pixel, meist nur eines, unterscheiden. Weitere Fehlerquellen sind natürlich die Bildrekonstruktion selbst und das spätere Zusammensetzen der rekonstruierten Teilbilder.

Für die Bestimmung eventueller Verschiebungen werden wiederum Kreuzkorrelationsmethoden verwendet. Hierbei treten Schwierigkeiten bei den im Linienkern aufgenommenen Bildern auf, da in ihnen die beobachteten Strukturen teilweise völlig anders aussehen als in den Linienflügeln und dem Kontinuum. Im Gegensatz zur Verarbeitung der Rohbilder stehen hier auch keine auf gleiche Weise rekonstruierten CCD 1-Bilder zur Verfügung, mit deren Hilfe stellvertretend für die CCD 2-Daten die Verschiebewerte bestimmt werden könnten. 
Das Programm zur automatischen Bestimmung der Verschiebungen wurde deshalb so geschrieben, dass all die Bilder, die eine strukturelle Ähnlichkeit mit Kontinuumsbildern haben, auf das 1. Bild des Scans korreliert werden. Die Bilder im Linienkern werden dagegen schrittweise jeweils auf ihr in Wellenlängenrichtung benachbartes Bild korreliert. Diese weisen bei den gewählten Beobachtungswerten (Kap. 4.2) hinreichende Ähnlichkeit auf, um die Verschiebung zwischen ihnen zuverlässig zu bestimmen. Dabei wird von beiden Linienflügeln her zum Linienkern hin vorgegangen, um „random walk“-Probleme durch Fehlerfortpflanzung gering zu halten. Abschließend stellt das Programm noch eine visuelle Kontroll- und Korrekturmöglichkeit zur Verfügung, um eine optimale Positionierung aller Einzelrekonstruktionen in einem gemeinsamen Datenblock zu gewährleisten. Es ist bemerkenswert, dass selbst bei einer Auflösung von $0,5^{\prime \prime}$, hier also fünf Pixeln, Verschiebungen um weniger als ein halbes Pixel durch visuelle Kontrolle schnell hintereinander abwechselnd auf dem Bildschirm dargestellter Bilder festgestellt werden können.

\subsubsection{Vergleich der Rekonstruktionsmethoden}

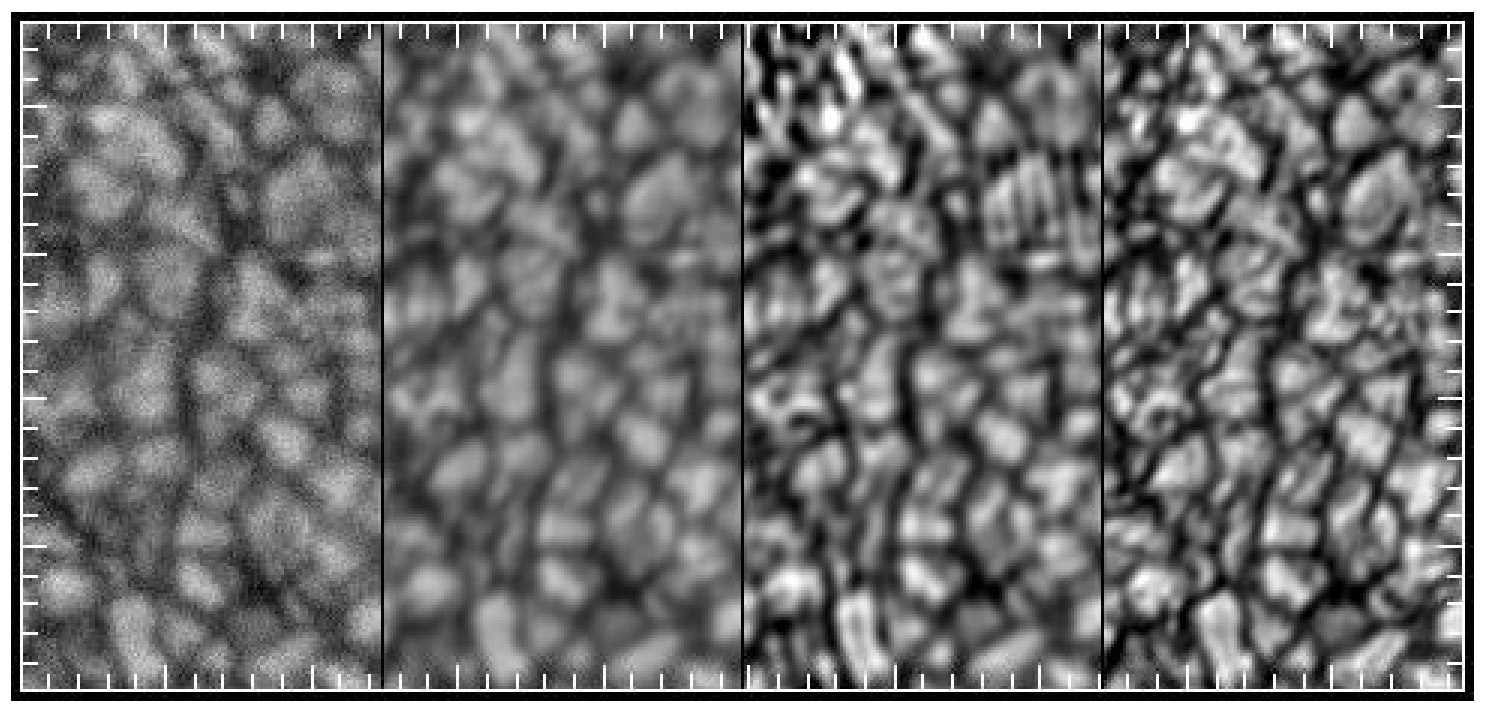

Abbildung 5.13: Vergleich der Rekonstruktionsergebnisse bei Scan s5_4. Gezeigt ist jeweils die 6. spektrale Position eines CCD 2-Scans, von links nach rechts das Rohbild nach der Frameselection-Methode, die einfache Entfaltung mit der Teleskopfunktion mit Rauschfilterung, die Phase Diversity Rekonstruktion und die Rekonstruktion nach dem Speckle-Verfahren. Der Abstand der Strichmarken entspricht 1".

Abschließend sollen noch einmal die Ergebnisse der verschiedenen Bildrekonstruktionsmethoden gegenübergestellt und verglichen werden. Abb. 5.13 zeigt die Bilder bzw. Rekonstruktionen an einer Wellenlängenposition noch nahe des Kontinuums. Abb. 5.14 dagegen zeigt die entsprechend berechneten Magnetfeldkarten. Erkennbar ist, dass schon die einfache Entfaltung der Bilder mit der Teleskopfunktion und die Rauschfilterung 


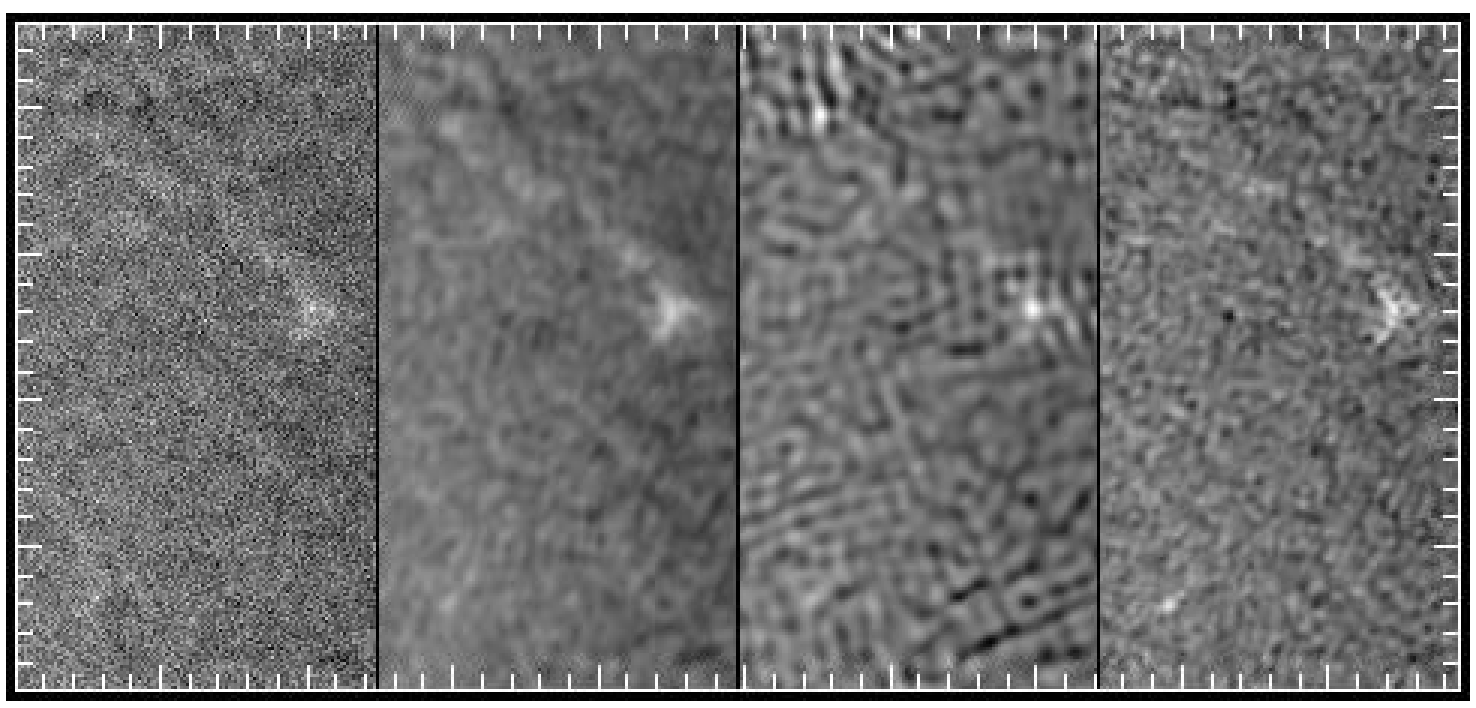

Abbildung 5.14: Vergleich der Rekonstruktionsergebnisse bei Scan s5_4. Gezeigt ist jeweils die Magnetfeldkarte, von links nach rechts berechnet aus den Rohbildern nach der FrameselectionMethode, aus den einfachen Entfaltungen mit der Teleskopfunktion mit Rauschfilterung, aus den Phase Diversity Rekonstruktionen und den Rekonstruktionen nach dem Speckle-Verfahren. Der Abstand der Strichmarken entspricht 1".

eine deutliche Verbesserung gegenüber der Verwendung der Rohbilder bzw. bei der Frameselection-Methode des jeweils besten Einzelbilds an einer spektralen Beobachtungsposition bringt. Besonders die Magnetfeldkarte zeigt deutlich mehr Strukturen, die bei Verwendung der Rohbilder im Rauschen untergehen.

Die Phase Diversity Methode bringt eine deutliche Verstärkung der Kontraste mit sich und hebt vereinzelt auch neue, vorher unsichtbare Feinstrukturen hervor. Zum Beispiel wird in Abb. 5.13 in der länglichen Granule am unteren Bildrand halblinks eine dunkle Linie sichtbar. Auch die Granule links darüber zeigt in der Phase Diversity Rekonstruktion deutliche Unterstruktur, die in den beiden linken Bildern nicht sichtbar ist. Dafür ist die Methode anfällig für Fehlberechnungen der Wellenfront, was zu Artefakten, also „falschen“ Strukturen im Bild führt, wie das Streifenmuster oben rechts im Bildfeld. Diese machen sich besonders in den Magnetfeldkarten bemerkbar, wo sich die Fehlstellen aus allen Wellenlängenpositionen aufsummieren. Während die Einzelrekonstruktionen nach dieser Methode durchaus noch ihren Wert haben können, werden die daraus berechneten Magnetfeldkarten völlig unbrauchbar für eine weitere Auswertung. Zudem schwankt die Qualität der Einzelrekonstruktionen stark mit dem Seeing. Bei den meisten spektralen Positionen bleibt die Auflösung deutlich hinter der der hier gezeigten Bilder zurück.

Als die mit Abstand erfolgreichste Methode hat sich die Speckle-Rekonstruktion erwiesen. Die Einzelrekonstruktionen zeigen, verglichen mit dem Ausgangsmaterial, eine bestechende Fülle von Feinstrukturen. Gleichzeitig ist die Methode sehr robust und we- 


\subsection{Bildrekonstruktion}

nig fehleranfällig, sie ergibt über den gesamten Scan auch bei leicht wechselndem Seeing Rekonstruktionen von beinahe gleichbleibender Qualität. Dies führt zu zuverlässiger Berechnung der Magnetfeldkarten, die sowohl an Empfindlichkeit wie auch an räumlicher Auflösung allen anderen gezeigten Ergebnissen weit überlegen ist.

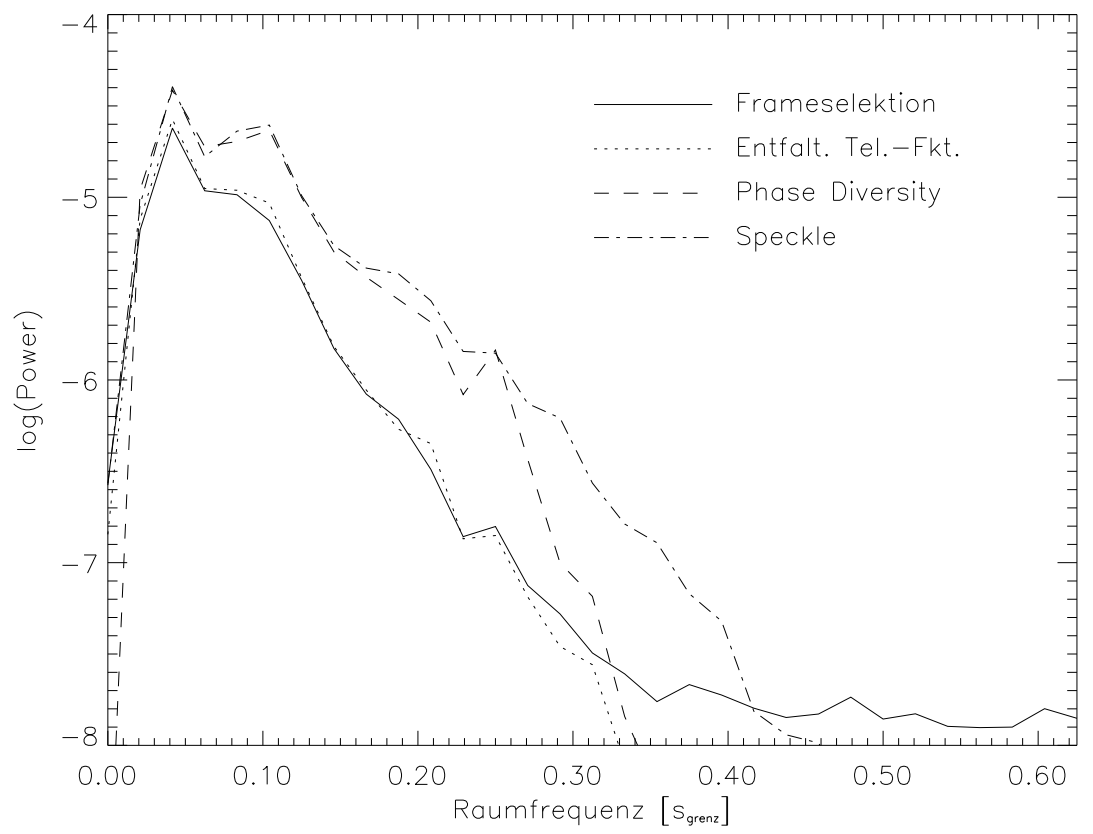

Abbildung 5.15: Powerspektren eines $96 \times 96$-Pixel-Ausschnitts des Scans s5_4 für die verschiedenen Rekonstruktions-Methoden. Die Raumfrequenzen sind in Einheiten der NyquistGrenzfrequenz angegeben.

Die Überlegenheit der Speckle-Methode gerade gegenüber der Phase Diversity Methode könnte darin begründet liegen, dass für die Berechnung einer Rekonstruktion nicht nur die an dieser Wellenlängenposition aufgenommenen Einzelbilder verwendet werden, sondern über die Speckle-Rekonstruktion des Breitbandkanals auch Informationen aus dem gesamten Scan in die Rekonstruktion einfließen können. Diese Breitband-SpeckleRekonstruktion ist aufgrund der hohen Redundanz der Phaseninformationen aus (in diesem Fall) 140 Einzelbildern in der Lage, auch geringste Strukturinformationen aus dem Rauschen herauszulösen. Das führt zu einer wesentlich genaueren (impliziten) Bestimmung der OTF jedes Einzelbilds durch Vergleich der Speckle-Rekonstruktion mit dem Einzelbild, als es die Phase Diversity Methode durch die (explizite) Berechnung der Wellenfront aus lediglich einem fokussierten und einem defokussierten Einzelbild allein leisten kann.

Auch quantitativ lassen sich die gemachten Aussagen überprüfen, indem für ein identisches Granulationsgebiet für die verschiedenen Rekonstruktionsmethoden die Powerspektren aufgetragen werden. Dies ist in Abb. 5.15 geschehen. Das Powerspektrum der 
Rohbilder geht bei einer Raumfrequenz, die etwa einer Strukturgröße von 0, 6 " entspricht, in das Rauschen über. Bei dem gepunktet dargestellten Powerspektrum des mit der Teleskopfunktion entfalteten und gefilterten Bilds ist die Wirkung des Rauschfilters bei eben dieser Raumfrequenz deutlich sichtbar. Dagegen ist die (bei diesen Raumfrequenzen geringe) Verstärkung der Strukturen durch die Entfaltung mit der Teleskop-OTF in der logarithmischen Auftragung kaum sichtbar.

Das gestrichelt dargestellte Powerspektrum der Phase Diversity Rekonstruktion zeigt deutlich die kontrastverstärkende Wirkung, reicht aber kaum zu kleineren Strukturen. Dagegen können mit der Speckle-Methode deutlich kleinere Strukturen bis zu 0,48" gefunden werden, wie die strichpunktierte Linie zeigt. 


\subsection{Auswertung der spektroskopischen Information}

In diesem Abschnitt soll beschrieben werden, wie aus den rekonstruierten Datensätzen, die mit einer der in Kap. 5.1 beschriebenen Methoden erzeugt wurden, die physikalischen Größen, insbesondere das Magnetfeld, berechnet werden. Bei den hier beschriebenen Beobachtungsdaten stehen nach der Bildrekonstruktion zu jedem Scan zwei Datensätze zur Verfügung, je einer für die beiden polarimetrischen Kanäle $\frac{1}{2}\left(I_{\lambda}+V_{\lambda}\right)$ und $\frac{1}{2}\left(I_{\lambda}-V_{\lambda}\right)$. Ehe sich durch Subtraktion bzw. Addition der beiden Kanäle die Stokes-I- und -VSpektren bilden lassen, sind weitere Schritte der Datenreduktion nötig. Anhand der Stokes-Spektren lassen sich später (Kap. 6) erste Aussagen über die beobachteten Magnetfelder treffen. Außerdem soll eine weitere Methode zur Bestimmung des magnetischen Flusses, die „Linienschwerpunktsmethode“ vorgestellt werden, die gleichzeitig Aussagen über die Geschwindigkeiten erlaubt, die in den beobachteten solaren Strukturen auftreten.

\subsubsection{Korrektur der Wellenlängenverschiebung}

Die Aufstellung der FPIs im parallelen Strahlengang hat zur Folge, dass die Wellenlänge maximaler Transmission von der Position im Bildfeld abhängig ist, da Lichtstrahlen, die zu unterschiedlichen Bildpositionen gehören, unter verschiedenen Winkeln durch die FPIs gehen. Dabei ergibt sich die maximale Transmissionswellenlänge ausschließlich aus dem Winkel, unter dem das Licht durch das die spektrale Aufösung ausmachende schmalbandige FPI 2 tritt (vgl. Kap. 3.2.2).

Bei guter optischer Justierung des Strahlengangs sollten die Lichtstrahlen von einem Bildpunkt in der Mitte des Bildfelds genau senkrecht auf das FPI 2 treffen. Sei dieser Punkt als Referenzwellenlänge angenommen, so unterscheiden sich die Transmissionswellenlängen von anderen Bildpunkten entsprechend Glg. 3.2 von der Referenzwellenlänge. Für ein nach der beschriebenen Datenreduktion verbleibendes Bildfeld von typischerweise $13^{\prime \prime} \times 23^{\prime \prime}$ sollten sich demnach Wellenlängendifferenzen zwischen Bildmitte und Bildecken von $0,56 \mathrm{pm}$ ergeben.

Nun ist allerdings zu bedenken, dass das ursprüngliche Lichtbündel, auf das die Beobachtungsanlage justiert wurde, von dem Polarimeter in der Höhe versetzt und seitlich aufgespalten wurde. Dies hat zur Folge, dass die Strahlen, die senkrecht auf das FPI 2 treffen, nicht mehr aus der Mitte der Bildfelder der beiden polarimetrischen Kanäle stammen, vielmehr die beiden Lichtbündel etwas unterschiedliche Wege durch das abbildende System und die FPIs nehmen. Während die Aufspaltung und der seitliche Versatz der beiden Bündel von wenigen Millimetern so gering ist, dass wegen der groß dimensionierten Linsen L 3 und L 4 ( $\varnothing 180 \mathrm{~mm}$ ) keine nennenswerten Abbildungsfehler zu erwarten sind, machen sich die unterschiedlichen Wege der beiden Bündel durch die FPIs bei der Transmissionswellenlänge durchaus bemerkbar. Es ist deshalb angebracht, anhand der Flatfieldscans für jeden Pixel in beiden polarimetrischen Kanälen die Linienkernposition 


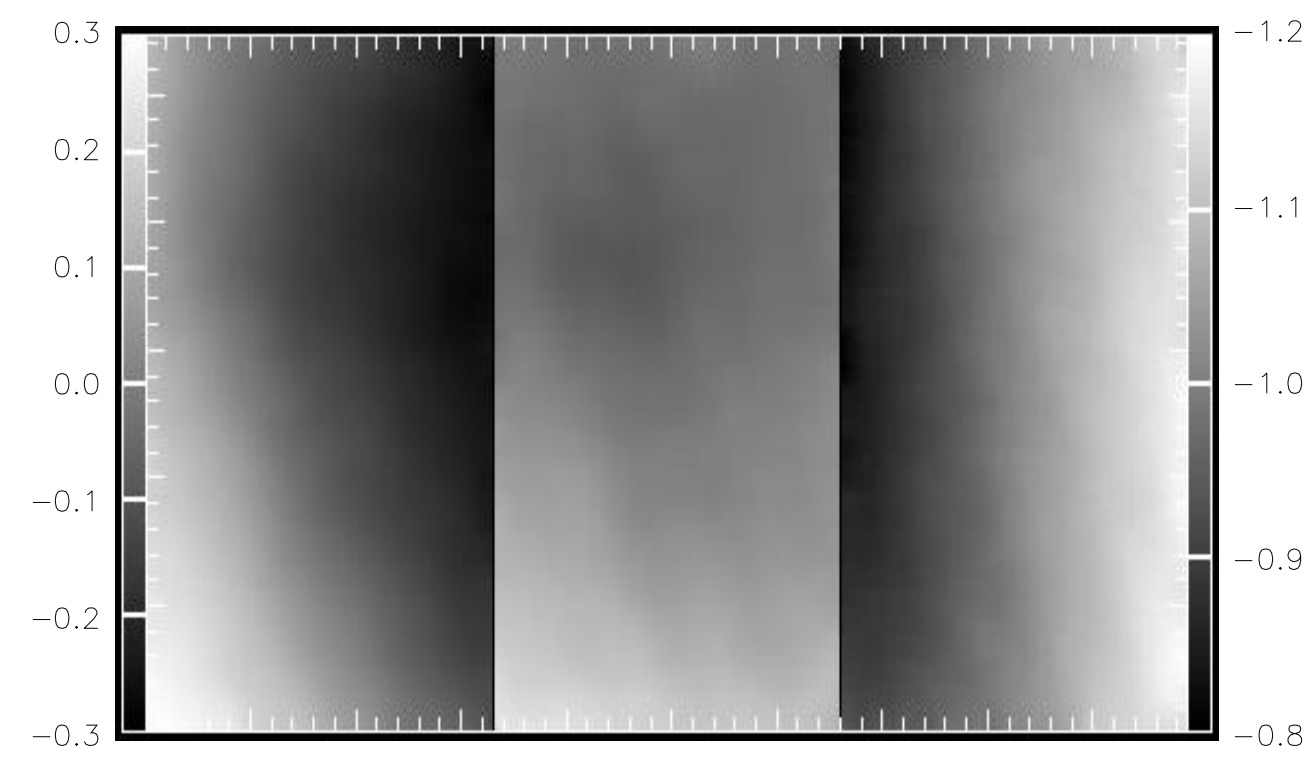

Abbildung 5.16: Differenz der Wellenlängen maximaler Transmission. Im linken Teilbild sind die Daten für den polarimetrischen Kanal $\frac{1}{2}\left(I_{\lambda}+V_{\lambda}\right)$ gezeigt, im mittleren für den anderen Kanal $\frac{1}{2}\left(I_{\lambda}-V_{\lambda}\right)$. Für diese beiden gilt die linke Skala in pm. Rechts die Wellenlängendifferenz zwischen den beiden Kanälen, es gilt die rechte Skala in pm. Der Abstand der Strichmarken entspricht $1^{\prime \prime}$.

zu messen und mit diesen Werten dann die Beobachtungsdaten zu korrigieren.

Zunächst werden also in einem Flatfieldscan je fünf Bilder an jeder Wellenlängenposition gemittelt, um das Rauschen zu verringern. Dann wird Pixel für Pixel die Lage der Spektrallinie mit dem Wert in der Mitte des Bildausschnitts des jeweiligen polarimetrischen Kanals verglichen. Die Verschiebung der beiden Spektrallinien gegeneinander wird durch Interpolation des Maximums der Kreuzkorrelationsfunktion der beiden Spektren subpixelgenau gemessen. Die Ergebnisse für die beiden Kanäle sind im linken und mittleren Bild in Abb. 5.16 gezeigt. Angegeben sind die Verschiebungen zwischen dem Spektrum jedes einzelnen Pixels und dem Spektrum des Pixels genau in der Bildmitte. In groben Zügen sind die Werte mit dem theoretisch zu erwartenden Verlauf vereinbar, insbesondere die Zahlenwerte von \pm 0,3 pm sind vernünftig. Geringe Abweichungen und Unregelmäßigkeiten lassen sich durch Ungenauigkeiten in der Justierung der optischen Komponenten erklären.

Im rechten Teilbild von Abb. 5.16 ist Pixel für Pixel die Differenz der Linienkernpositionen in den beiden polarimetrischen Kanälen angegeben. Auch hier sind die gemessenen Werte von ca. $1 \mathrm{pm}$ realistisch. Es sei angemerkt, dass die gezeigten VerschiebungsKarten über 21 Pixel geglättet wurden, da kleinskalige Variationen z. T. durch Rauschen in den Flatfieldbildern verursacht werden können, und außerdem nicht klar ist, ob sie als repräsentativ für einen ganzen Beobachtungstag angesehen werden dürfen.

Mit Kenntnis dieser Verschiebungswerte können nun in den Beobachtungsdaten Pixel für Pixel die Spektren auf eine einheitliche Referenzwellenlänge verschoben werden. Da 
es sich bei den Verschiebungswerten um Bruchteile von diskreten Wellenlängenpositionen handelt, musste ein Interpolationsverfahren gefunden werden, welches subpixelVerschiebungen ohne Verfälschung der im Spektrum enthaltenen Informationen gewährleistet. Hier hat sich ein Verfahren im Fourierraum bewährt. Dazu wird jedes einzelne Spektrum zunächst in ein längeres Datenfeld so eingebettet, dass die Intensitäten an den Rändern des Spektrums glatt auf einen gemeinsamen Wert gebracht werden können (Apodisation). Das so verlängerte Spektrum wird fouriertransformiert, dann um einen Faktor 100 über die Nyquist-Wellenzahl hinaus verlängert und in den Wellenlängenraum zurücktransformiert. Nun kann eine Verschiebung um den ganzzahligen Wert, der dem geforderten Verschiebungswert am nächsten ist, durchgeführt werden. Danach wird das Spektrum wieder im Fourierraum um den Faktor 100 verkleinert und im Wellenlängenraum der ursprüngliche Ausschnitt wieder hergestellt. Mit diesem Verfahren sind zuverlässige Verschiebungen der Spektren um 1/100 Wellenlängenposition möglich. Noch feinere Verschiebungen wären natürlich (durch höhere Vergrößerungsfaktoren) möglich, sind aber hier im Rahmen der Messgenauigkeit nicht nötig.

\subsubsection{Korrektur der Filtertransmissionskurve}

Die zu diesem Verarbeitungsstadium vorliegenden Spektren an den einzelnen Bildpunkten enthalten noch die Transmissionskurve des Vorfilters IF 2 (Abb. 5.17). Diese muss mit Hilfe der aufgenommenen Scans mit der Kontinuumslampe reduziert werden. Per Definition enthalten diese Scans keine Spektrallinie, mit deren Hilfe eine Wellenlängenkalibration des Scans durchgeführt werden könnte. Wegen der unbekannten Drift des FPI2 ist also eine genaue, relative Verschiebung der Wellenlänge zu den Beobachtungsdaten unbekannt. Deshalb sind in Abb. 5.17 auch nur die FPI-Positionen auf der x-Achse angegeben. Da die Transmissionskurve des Filters aber in dem abgetasteten Bereich der Spektrallinie sehr flach und ohne deutliche, kleinskalige Struktur ist, ist eine exakte Positionierung in Wellenlängenrichtung auch nicht nötig. Die maximalen Abweichungen von einer Gerade betragen $\pm 0,3 \%$. Auch

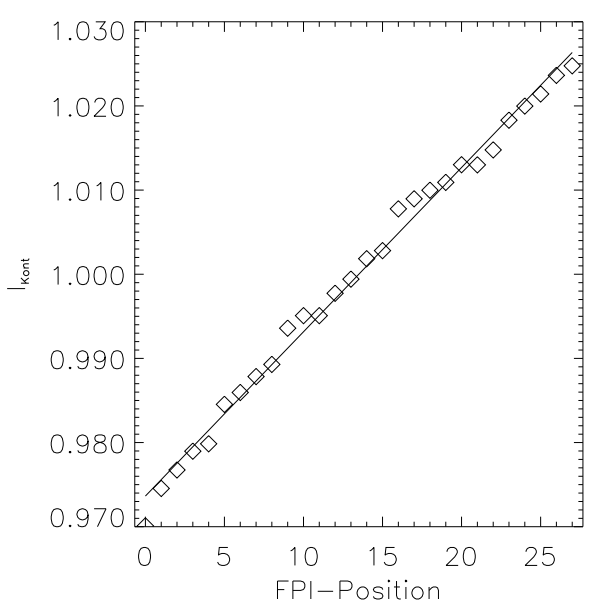

Abbildung 5.17: Normierte Transmissionskurve des Vorfilters IF 2 $(630,25 \mathrm{~nm})$. Die $\diamond$ bezeichnen die über das Bildfeld gemittelten Messwerte, die durchgezogene Linie ist die Regressionsgerade.

die im ungünstigsten Fall bei einer falschen Abstimmung von Beobachtungs- und Kontinuumsscan um ein bis drei FPI-Positionen auftretenden Fehler sind 0,3\%. Aus demselben Grund ist es auch nicht erforderlich, die auch in den Kontinuumsscans vorhandene 
Blauverschiebung über das Bildfeld zu berücksichtigen. Also wurde nach Abzug des zugehörigen Dunkelsignals durch Mittelung eines Kontinuumsscans über das gesamte Bildfeld eine mittlere Transmissionskurve des Filters berechnet, durch die dann die Spektren der Beobachtungsdaten pixelweise dividiert wurden. Der leichte, konstante Gradient der Transmissionskurve hat im Übrigen bei einer unterschiedlichen Wellenlängenpositionierung der FPIs bei Datenaufnahme und Kontinuumsscan wegen der Normierung der Kurve keinen fehlerhaften Einfluss auf die Datenreduktion.

Bei wesentlich schmaleren Vorfiltern oder wenn im abgetasteten Wellenlängenbereich starke Krümmungen der Transmissionskurve vorhanden sind, ist eine genaue Positionierung der Transmissionskurve bezüglich der Beobachtungsdaten in Wellenlängenrichtung allerdings wichtig. Dann stehen aber mit eben diesen Krümmungen auch wiederum geeignete Hilfsmittel für eine Wellenlängenkalibrierung der Scans zur Verfügung (vgl. Krieg (1999 a), dort S. 34).

\subsubsection{Stokes-I- und -V-Spektren}

Durch Abziehen der Daten aus den beiden Kanälen $\frac{1}{2}\left(I_{\lambda}+V_{\lambda}\right)$ und $\frac{1}{2}\left(I_{\lambda}-V_{\lambda}\right)$ voneinander ergibt sich das Stokes-V-Profil $V_{\lambda}$, durch Addition das Stokes-I-Profil $I_{\lambda}$. Die V-Signale werden auf die Kontinuumsintensität normiert, indem sie durch ein Stokes-I-Bild im Kontinuum geteilt werden. Um hierbei Fehler durch Reste von Rauschen oder Unvollkommenheiten der Bildrekonstruktion zu vermeiden, werden dazu die Bilder mehrerer FPI-Positionen außerhalb der Spektrallinie zu einem Bild gemittelt und dieses dann noch über $3 \times 3$ Pixel geglättet.

Nun stehen im Prinzip für jedes Pixel des zweidimensionalen Bildfelds Spektren zur Verfügung, die für die physikalische Analyse der Parameter der Sonnenatmosphäre genutzt werden können. Da die räumliche Auflösung der rekonstruierten Datenblöcke in den besten Scans 0,4" - 0,5" beträgt, dürfen bei einer Abtastrate von 0,1" /Pixel bei der Betrachtung der Spektren für einzelne Punkte im Bild guten Gewissens die Spektren einzelner, benachbarter Pixel gemittelt werden, um das Signal-zu-Rausch-Verhältnis (SNR) zu verbessern. Hierbei sind Mittelungen über $3 \times 3$ Pixel ohne weiteres zulässig, ohne dass eine Verschlechterung der Auflösung zu befürchten ist.

\section{Glättung der Profile}

Trotz dieser Mittelung zeigen die Spektren teilweise immer noch einzelne Spitzen oder Einbrüche, die nicht sonnenphysikalischer Natur sind. Dies kann z. B. durch Rekonstruktionen an einzelnen FPI-Positionen hervorgerufen werden, die wegen kurzzeitig schlechteren Seeings nicht die hohe räumliche Auflösung der benachbarten FPI-Positionen haben. In diesem Fall kann magnetisches Signal aus einzelnen Pixeln heraus gestreut und in andere Pixel hinein gestreut werden. Dasselbe passiert bei Unvollkommenheiten der Bildrekonstruktion oder bei kleinen Fehlern beim Zusammensetzen der Teilbilder oder beim Aufeinanderschieben der fertig rekonstruierten Bilder zu einem gemeinsamen Da- 
tenblock. Solche einzelnen Spitzen sind aber von geringer Bedeutung und dürfen im Zweifelsfall durch eine Glättung der Spektren in Wellenlängenrichtung abgemildert oder beseitigt werden.

\section{Flatfield-Korrektur 2. Ordnung}

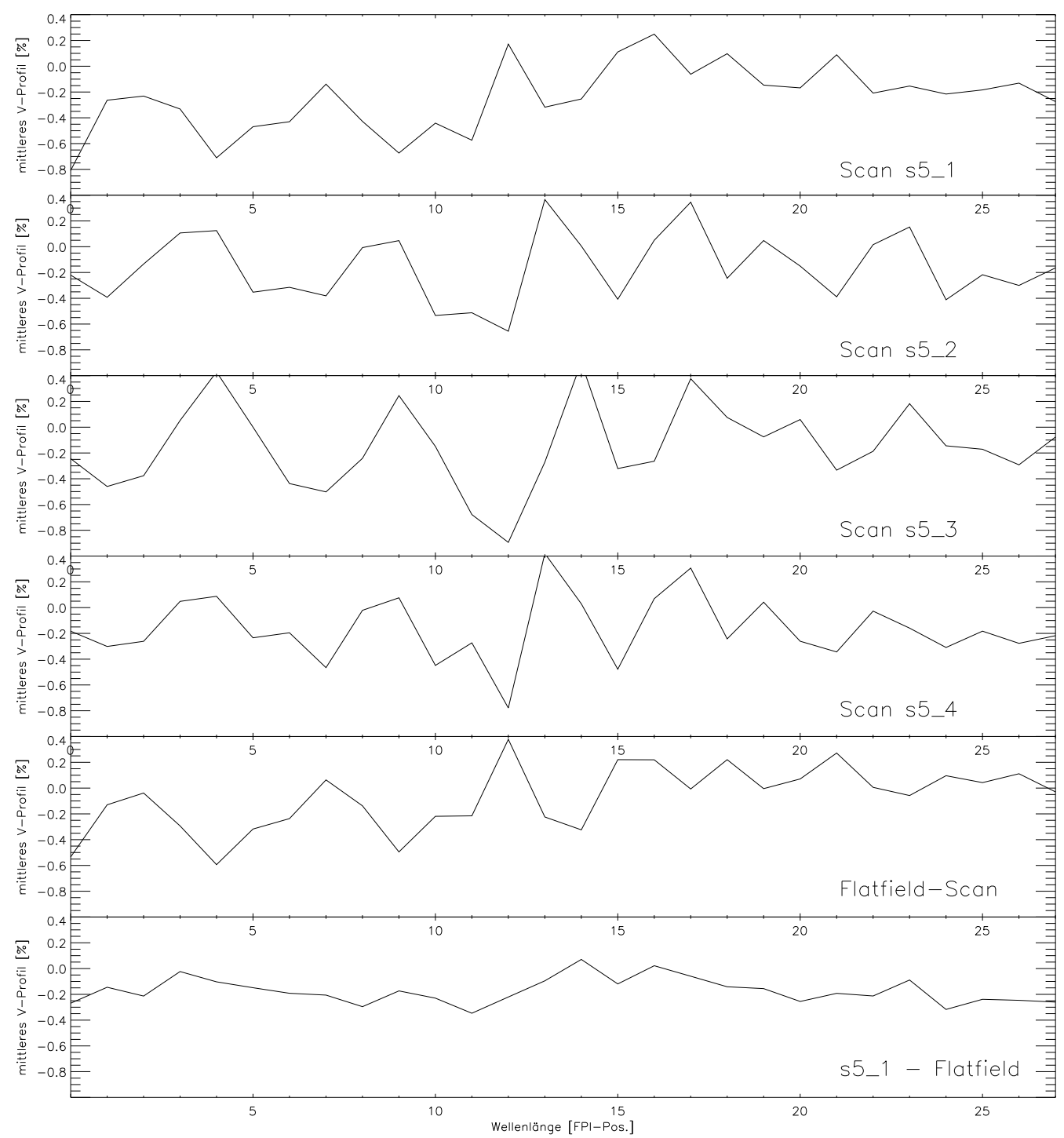

Abbildung 5.18: Rest-Stokes-V-Profile (in \% der Kontinuumsintensität des I-Profils) aus unmagnetischen Granulationsgebieten (oberste vier Reihen) und Flatfieldscan (5. Reihe). In der untersten Reihe ist das verbleibende Profil für den Scan s5_1 gezeigt, nachdem die 2nd-orderKorrektur mit dem Flatfield-Profil durchgeführt wurde.

Berechnet man über größere, offensichtlich unmagnetische Gebiete der Sonnenoberfläche oder über Ausschnitte aus den Flatfieldscans mittlere Stokes-V-Profile, so sollte man sehr rauscharme, praktisch konstant bei null liegende Profile erwarten. Dies ist allerdings in der Praxis nicht der Fall, es bleiben Signale von einigen Promille des Stokes-I-Signals 
übrig. Eine genauere Betrachtung zeigt, dass diese „Rest-V-Signale“ zwar über das Bildfeld variieren, aber von Scan zu Scan ungefähr gleich bleiben. Sie sind sowohl im Flatfieldscan als auch in den Scans mit ruhiger Granulation zu finden. Dass es sich bei diesen Profilen um reproduzierbare, für den entsprechenden Bildausschnitt typische Signale handelt, wird auch durch die Tatsache nahegelegt, dass diese Restprofile verschiedener Scans genau dieselbe grobe Verschiebung der Wellenlänge infolge der Drift des FPI 2 aufweisen wie die Linienkerne. Anhand des Flatfieldscans lassen sich also „Flatfield-Profile 2. Ordnung" erzeugen, die von den beobachteten Profilen abgezogen werden könnnen. Dabei muss natürlich auf die richtige Wellenlängenabstimmung zwischen den Daten und dem Flatfield-Profil geachtet werden. Da die auf die Kontinuumsintensität des entsprechenden Scans normierten V-Signale verwendet werden, spielen unterschiedliche mittlere Helligkeiten der einzelnen Scans hier keine Rolle.

Abb. 5.18 zeigt für einen $50 \times 50$ Pixel umfassenden Ausschnitt aus den Bildfeldern von den vom 22.10.99 verfügbaren Granulationsscans und dem Flatfieldscan das mittlere Stokes-V-Profil. Deutlich ist die Ähnlichkeit der Profile zu erkennen, wobei die Verschiebungen in Wellenlänge durch die von Scan zu Scan unterschiedliche Drift von FPI2 verursacht werden. Die unterste Auftragung zeigt das Profil von Scan s5_1 nach Abzug des Flatfield-Profils, deutlich ist der Erfolg der Korrektur zu erkennen.

Es muss hier aber deutlich gemacht werden, dass diese geringe Korrektur von wenigen Promille in den Datenscans bei einzelnen Pixeln im Rauschen untergeht. Allerdings gewinnt sie für statistische Auswertungen, in denen mittlere Profile über sehr viele Pixel bestimmt werden, stark an Bedeutung.

Ein ähnliches Verfahren des „2nd-order flatfielding“ wird bei Sütterlin (1996), Navarro (1999) und Sánchez Almeida \& Martínez Pillet (1994) beschrieben.

\section{Detektionsgrenze der Magnetfelder}

Das resultierende Rauschniveau in den über $3 \times 3$ Pixel gemittelten Stokes-V-Spektren ist (bei der Rekonstruktionsmethode nach Kap. 5.1.7) ca. $\pm 0,5-0,7 \%$ der Kontinuumsintensität des Intensitätsprofils und lässt sich bei den in Wellenlängenrichtung geglätteten Spektren auf ca. $\pm 0,3 \%$ verringern. Verglichen mit den unrekonstruierten, lediglich durch Frameselection ausgewählten Rohbildern ist das schon eine deutliche Verbesserung. Bei diesen liegt das Rauschniveau bei $\pm 1 \%$ für die ungeglätteten Profile bzw. bei $\pm 0,6 \%$ für die geglätteten Profile.

Obwohl für die meisten Stellen im Bildfeld, in denen kein deutliches Anzeichen magnetischer Aktivität zu finden ist, die V-Profile praktisch konstant sind mit den oben genannten Rauschniveaus, finden sich immer wieder Stellen, an denen deutliche Schwankungen und Trends der Profile von der Größenordnung $\pm 1 \%$ erkennbar sind, die weder nach reinem gaußverteilten Rauschen aussehen, noch Ähnlichkeit mit typischen V-Profilen haben. Da diese Signale im Bereich der Detektionsgrenze liegen, soll ihnen keine sonnen- 


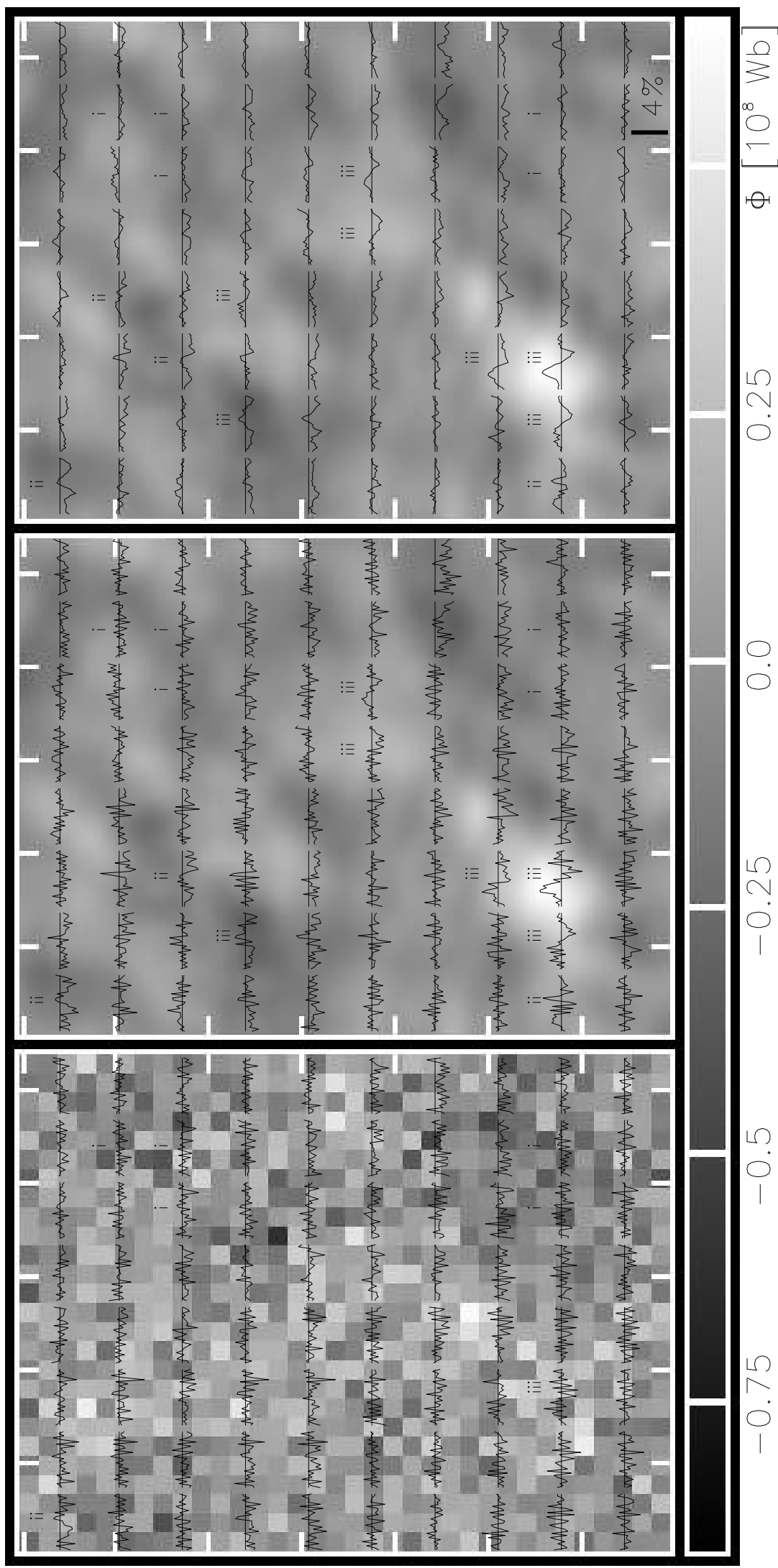

Abbildung 5.19: Detektionslimit für Magnetfelder in Stokes-V-Spektren. Eine genauere Beschreibung der Abbildung wird im Text gegeben. Die Magnetfelder sind in Wb durch ein Pixel angegeben, ebenso in allen folgenden Abbildungen. Der Abstand der Strichmarken entspricht $1^{\prime \prime}$. 
physikalische Bedeutung beigemessen werden. Die Ursachen sind vermutlich in geringen Fehlern bei der Bildrekonstruktion, dem Zusammensetzen der Teilbilder und Aufeinanderschieben der rekonstruierten Bilder zu suchen, eventuell auch im Zusammentreffen von gerade eben detektierbaren Magnetfeldern und Rauschen. Einige Profile zeigen auch mehr oder weniger konstante Offsets von bis $\mathrm{zu} \pm 0,7 \%$, deren Ursache vermutlich in geringen Fehlern bei der Flatfieldprozedur liegt, und die auch durch das 2nd-order Flatfielding nicht behoben werden können. Da sie auch in den unrekonstruierten Bildern auftreten, scheiden Fehler bei der Bildrekonstruktion als Ursache aus.

Häufig finden sich aber auch Profile von $\pm 0,5-1 \%$, die sich klar als typische Stokes-VProfile identifizieren lassen. Als zusätzlicher „Vertrauensbeweis“ kann die in Kap. 5.2.5 beschriebene Linienschwerpunktsmethode zur Bestimmung der Magnetfelder herangezogen werden. Wenn diese ebenfalls an der betreffenden Stelle des Bildfelds ein schwaches Magnetfeld der richtigen Polarität anzeigt, kann man von einem „nachgewiesenen Magnetfeld" sprechen. Weiterhin ist festzustellen, dass die Bewertung solcher Stokes-VSignale an der Detektionsgrenze durch die Glättung der Profile in Wellenlängenrichtung erleichtert wird.

In Abb. 5.19 sind all die oben geschilderten V-Profil-Typen zu finden. Gezeigt ist jeweils eine Magnetfeldkarte, die mit der Linienschwerpunktsmethode (Kap. 5.2.5) berechnet wurde, über die Stokes-V-Profile aufgetragen sind, und zwar immer dort im Bildfeld, wo das entsprechende Profil berechnet wurde. Der Bildausschnitt ist aus dem Scan s5_4 vom 22.10.99 entnommen und soll später im Ergebniskapitel noch genauer besprochen werden. Im ersten Teilbild sind die Ergebnisse für die Rohdaten aus der FrameselectionMethode (Kap. 5.1.3) gezeigt, wobei die Profile über je $3 \times 3$ Pixel gemittelt wurden, im zweiten Teilbild dasselbe für die Speckle-Methode (Kap. 5.1.7). Im dritten Teilbild sind die Profile zusätzlich in Wellenlängenrichtung über drei Positionen geglättet worden. Die Skala unten rechts entspricht einer Stärke des V-Signals von 4\%. Mit i sind Profile gekennzeichnet, die kein Magnetfeld, sondern nur „sauberes“ Rauschen zeigen, mit ii nicht genauer definierbare Signale am Detektionslimit und mit i i solche Profile, die eine deutliche Signatur solarer Magnetfelder tragen und bei denen zusätzlich die Linienschwerpunktsmethode einen entsprechenden Hinweis gibt. Man beachte z. B. den Polaritätswechsel zwischen 2. und 4. Profil in der 4. Reihe von oben, der zu dem Polaritätswechsel im Linienschwerpunktsbild passt.

Zusammengefasst lässt sich also sagen, dass in den rekonstruierten Daten in Einzelfällen bei genauer, visueller Inspektion der Profile und durch Vergleich mit der Linienschwerpunktsmethode Stokes-V-Signale von $\pm 0,5 \%$ nachgewiesen werden können. Magnetfelder mit einem Polarisationsgrad von $\pm 1-1,5 \%$ lassen sich dagegen immer zweifelsfrei identifizieren. Felder dieser Größenordnung lassen sich dagegen in den unrekonstruierten Daten kaum nachweisen. Einen weiteren Hinweis auf die „Echtheit“ detektierter Magnetfelder erhält man, wenn sich in aufeinanderfolgenden Scans ähnliche Signale an gleicher Stelle finden. 


\subsection{Auswertung der spektroskopischen Information}

\section{Geschwindigkeiten aus Stokes-V-Profilen}

Aus den Stokes-V-Profilen lassen sich prinzipiell auch die Geschwindigkeiten der strömenden Gase in den magnetischen Anteilen der Atmosphäre entnehmen, indem die Wellenlängenposition des Nulldurchgangs des Profils bestimmt und als Dopplerverschiebung gedeutet wird. Diese Werte werden dann häufig als „zero crossing“-Geschwindigkeiten bezeichnet. Bei den vorliegenden Daten ist jedoch eine zuverlässige Bestimmung dieser Geschwindigkeiten aufgrund des geringen SNR in den V-Profilen nicht möglich. Weiterhin erschweren die oben beschriebenen geringen Offsets einzelner Profile die genaue Bestimmung des Nullniveaus der Profile. So ist auf diese Weise bei den vorliegenden Daten nur ein grober Hinweis auf die Stärke und das Vorzeichen eventueller Strömungen zu bekommen.

\subsubsection{Magnetfeldkarten aus Stokes-V-Amplituden}

Aus den Stokes-V-Profilen lassen sich erste Abschätzungen treffen, wo sich im Bildfeld magnetische Strukturen befinden und ob es sich um stärkere oder schwächere Felder handelt. Wie schon in Kap. 4.1 gezeigt wurde, ist gerade für schwächere Felder die Aufspaltung des V-Signals ein schlechtes Maß für die Magnetfeldstärke $B$ und zudem noch beim Vorhandensein von Rauschen schlecht zu bestimmen. Gerade für schwache Felder kommt eher schon die Stärke des V-Signals in Frage, um die Anwesenheit und ungefähre Stärke der Felder zu bestimmen. Zu beachten ist natürlich, dass ab ca. 0,1 T die Sättigung der V-Amplitude eintritt.

Indem für jeden Pixel des Bildfelds die Amplitude des V-Signals bestimmt wird, können halb-quantitative Magnetfeldkarten angefertigt werden. Eine genaue visuelle Inspektion dieser Karten und der

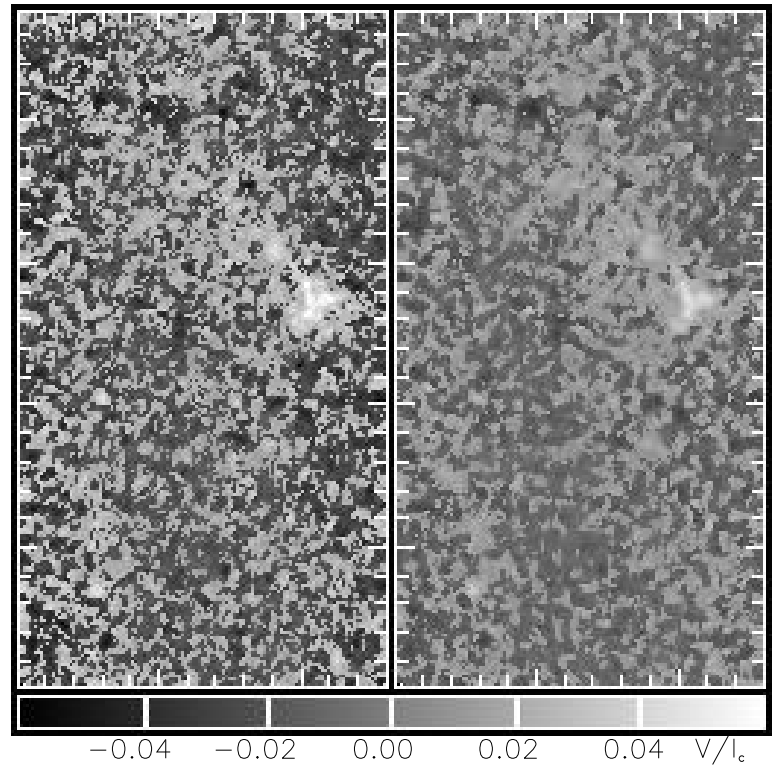

Abbildung 5.20: Halb-quantitative Magnetfeldkarte. Stokes-V-Amplitude für Scan s5_4. Links aus den über $3 \times 3$ Pixel gemittelten Profilen, rechts zusätzlich noch in Wellenlängenrichtung über drei Positionen geglättet. Der Abstand der Strichmarken entspricht 1". Stokes-V-Profile an diesen Stellen lässt deutlich erkennen, wo das Signal durch Rauschen bestimmt ist und wo sich wirklich Magnetfelder auf der Sonnenoberfläche nachweisen lassen.

Ein Beispiel zeigt Abb. 5.20, in der für den Scan s5_4 die Karte der Stokes-V-Amplitude gezeigt ist, links für die über $3 \times 3$ Pixel gemittelten Profile, rechts für Profile, die zusätzlich in Wellenlängenrichtung über 3 Positionen gemittelt wurden. Sichtbar sind 
magnetische Gebiete am rechten Bildrand und unten links und schwache Felder entgegengesetzter Polarität oben links. Der Rest des Bildfelds zeigt zunächst keine deutlichen Magnetfelder. In diesen Regionen wird sichtbar, dass neben dem dominierenden Rauschen geringe systematische Fehler vorliegen, die in Form schwacher, von unten rechts nach oben links verlaufender, breiter Streifen sichtbar sind. Aufgrund ihrer Ähnlichkeit zu den Intensitätsverläufen der einzelnen Flatfieldaufnahmen im Bereich der Spektrallinie ist ihre Ursache wohl in Unvollkommenheiten des Flatfieldings der Daten zu suchen. Hier sind diese Einflüsse aber gegenüber dem allgegenwärtigen Rauschen so gering, dass diesen systematischen Fehlern keine weitere Aufmerksamkeit geschenkt werden soll.

Weitere Magnetfeldkarten und eine genauere Untersuchung der Daten erfolgen in Kap. 6. Diese soll aber im Wesentlichen anhand der quantitativen Karten des magnetischen Flusses geschehen, die mit der im folgenden Abschnitt erläuterten Methode berechnet werden.

\subsubsection{Linienschwerpunktsmethode}

Eine etwas andere Methode als die direkte Betrachtung der Stokes-V-Profile zur Bestimmung der Magnetfelder wird von Semel (1967)] beschrieben. Das Problem bei Stokes-VProfilen ist das geringe SNR, das häufig eine zuverlässige Bestimmung von Aufspaltung und Amplitude im Rauschen untergehen lässt. Stattdessen werden die Spektren $\frac{1}{2}\left(I_{\lambda} \pm V_{\lambda}\right)$ direkt genutzt, um die Aufspaltung zu messen. Das SNR dieser Spektren kann um Größenordnungen besser sein als in den V-Signalen. Die Methode beruht darauf, dass die V-Signale die in beiden Kanälen identischen I-Signale überlagern. Dadurch werden die beiden Linienprofile asymmetrisch, das eine auf der kurzwelligen Seite, das andere auf der langwelligen. Diese Asymmetrie bewirkt, dass sich die Flächenschwerpunkte der Linienprofile ebenfalls verschieben. Der halbe Abstand $\Delta \lambda_{\mathrm{S}, 1 / 2}$ der Schwerpunkte der beiden Profile ist dann proportional zur mittleren magnetischen Flussdichte in Beobachtungsrichtung (vgl. auch Glg. 2.4),

$$
|B| \cos \gamma=\frac{4 \pi c m_{\mathrm{e}}}{e} \frac{\Delta \lambda_{\mathrm{S}, 1 / 2}}{\lambda_{0}^{2} \cdot g_{\mathrm{eff}}},
$$

bzw. zum magnetischen Fluss $\Phi$ im Auflösungselement $A$ in Beobachtungsrichtung mit

$$
\Phi=|B| \cos \gamma \cdot A \text {. }
$$

$\gamma$ sei der Winkel zwischen Sichtlinie und Richtung des Magnetfelds.

Die Koordinaten der Schwerpunkte der Linienprofile werden anhand der Guldin'schen Regeln (z. B. in Smirnow (1990)] berechnet. Dazu wird zuerst das lokale Kontinuumsniveau abgezogen und das Linienprofil dann mit -1 multipliziert, so dass die Absorptionslinie wie eine Emissionslinie erscheint. Für die Lage des Schwerpunkts in Wellenlängenrichtung $\lambda_{\mathrm{S}}$ eines solchen Linienprofils gilt:

$$
\lambda_{\mathrm{S}}=\frac{\int \lambda \cdot I(\lambda) d \lambda}{\int I(\lambda) d \lambda}
$$




\subsection{Auswertung der spektroskopischen Information}

Dabei wird bei einem diskreten Datenfeld wie den vorliegenden Spektren das Integral durch eine Summation der Werte an den einzelnen Messpunkten ausgeführt. Bei Beobachtungen mit der Fe I-Linie bei 630,25 nm ist zu beachten, dass die benachbarte, atmosphärische $\mathrm{O}_{2}$-Linie nicht in die Summation einbezogen wird, da sie die Berechnung des Schwerpunkts verfälschen würde.

Diese Schwerpunktsmethode ist zudem unabhängig von der Verschmierung durch das spektrale Apparateprofil (Airyfunktion). Sie misst allerdings einen Wert, der über die Entstehungshöhe der Spektrallinie in der Sonnenatmosphäre gemittelt ist.

Es muss hier hervorgehoben werden, dass mit dieser Methode lediglich die über das Auflösungselement $A$ gemittelte Sichtlinienkomponente des Magnetfelds bestimmt werden kann. Es kann also keine Aussage darüber getroffen werden, wie sich das magnetische Signal über das Aufösungselement verteilt, also auch nicht über den Füllfaktor und die wahre Magnetfeldstärke in nicht aufgelösten magnetischen Elementen. Es ist deshalb sinnvoller, von einer Bestimmung des magnetischen Flusses innerhalb des Auflösungselements (Glg. 5.18) zu sprechen und als Messgrößen eben diesen Fluss anzugeben. Es sind deshalb im Folgenden immer die magnetischen Flüsse in einem Gebiet der Sonnenoberfläche angegeben, das einem Kamerapixel in der Abbildung der Oberfläche entspricht; das sind $5580 \mathrm{~km}^{2}$.

Rees \& Semel (1979) zeigten, dass die Linienschwerpunktsmethode unabhängig vom Aufspaltungsmuster ist, also auch für Spektrallinien geeignet ist, die den anomalen Zeeman-Effekt zeigen. Sie reagiert nicht auf die Amplitudensättigung der V-Amplitude bei vollständiger Aufspaltung (vgl. Kap. 4.1) und ermöglicht für eine breite Auswahl von Spektrallinien und sonnenatmosphärischen Szenarien eine Bestimmung des magnetischen Flusses mit einer Genauigkeit von besser als $10 \%$.

In der Abb. 5.21 ist für den Scan s5_4 links die halb-quantitative Magnetfeldkarte aus der V-Amplitude der in Wellenlängenrichtung geglätteten Profile gezeigt (wie in Abb. 5.20 rechts) und zum Vergleich dazu rechts die Karte des magnetischen Flusses in einem Pixel aus der Linienschwerpunktsmethode. Beide Karten sind aus demselben Datensatz erzeugt, der mit der Speckle-Methode (Kap. 5.1.7) rekonstruiert wurde. Auffällig ist die bessere räumliche Aufösung der Flusskarte aus der Linienschwerpunktsmethode von $0,4^{\prime \prime}-0,5^{\prime \prime}$ und das geringere Rauschniveau. Die feinsten Strukturen in dieser Karte sind auch hier als Rauschen zu betrachten, die (hier nicht gezeigten) Stokes-V-Profile zeigen für die allermeisten Stellen im Bild kein merkliches Anzeichen für Magnetfelder. Aber fast allen sich deutlicher abhebenden hellen und dunklen Stellen lässt sich zuverlässig ein Magnetfeld zuordnen, genaueres wird später in Kap. 6.1 besprochen. Weiterhin fällt auf, dass das systematische Restsignal in Form breiter Streifen, das in den V-AmplitudenKarten zu sehen ist, hier nicht auftritt. Die Methode ist offenbar auch gegen geringe Fehler bei der Flatfieldprozedur unempfindlich. Das Detektionslimit in diesen Karten muss mit etwa $5 \cdot 10^{7} \mathrm{~Wb}$ angenommen werden. Zwar ist das RMS in den unmagnetischen Gebieten der Flusskarte nur $2 \cdot 10^{7} \mathrm{~Wb}$, jedoch sind erst Strukturen, die einen 


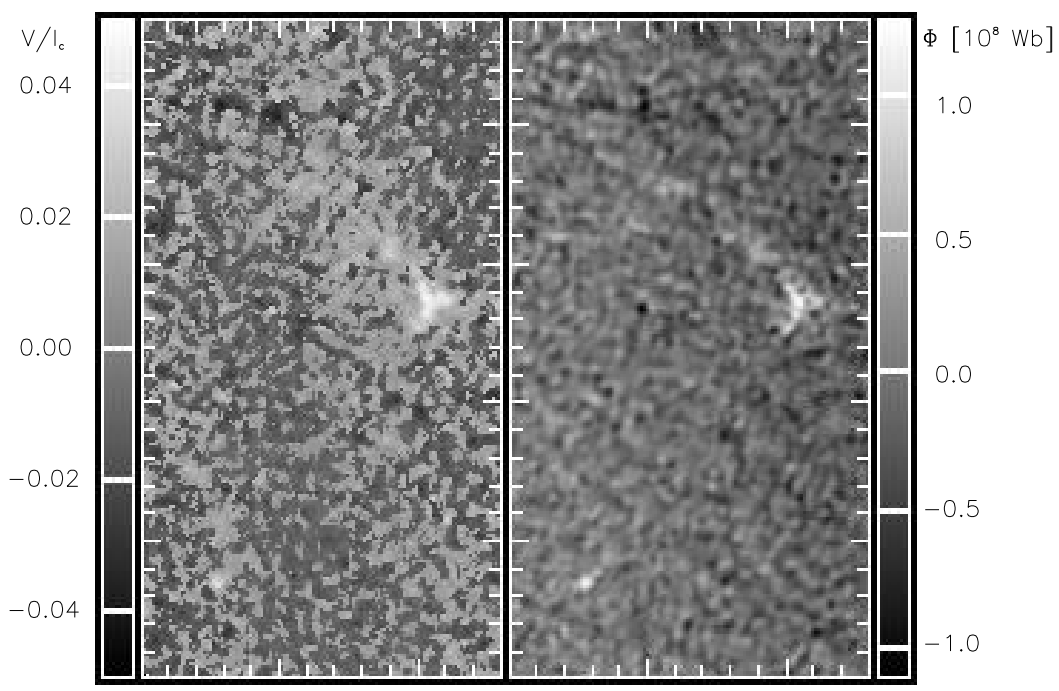

Abbildung 5.21: Rechts: Karte des magnetischen Flusses in einem Pixel, die für den Scan s5_4 aus der Aufspaltung der Linienschwerpunkte berechnet wurde. Links: Zum Vergleich dazu die halb quantitative Magnetkarte aus den geglätteten Profilen aus Abb. 5.20. Der Abstand der Strichmarken entspricht $1^{\prime \prime}$.

Fluss von mehr als $5 \cdot 10^{7} \mathrm{~Wb}$ aufweisen, immer eindeutig als magnetische Strukturen zu identifizieren. Aufgrund des besseren SNR in den polarimetrischen Kanälen $\frac{1}{2}\left(I_{\lambda} \pm V_{\lambda}\right)$ als im Stokes-V-Signal ist diese Methode zur Bestimmung magnetischer Strukturen die empfindlichere. Deshalb können im Einzelfall, z. B. wenn sich ähnliche Strukturen in aufeinanderfolgenden Scans an gleicher Stelle zeigen, auch solche magnetischen Strukturen als „echt“ bezeichnet werden, in denen das Stokes-V-Signal keine deutliche, sich vom Rauschen abhebende typische Signatur zeigt. Beispiele solcher Strukturen finden sich zahlreich im Ergebniskapitel (Kap. 6, u. a. in Abb. 6.6, 6.11 und 6.12).

Weiterhin lassen sich die Linienschwerpunkte des Stokes-I-Signals, die annähernd das arithmetische Mittel der Schwerpunktspositionen der beiden polarimetrischen Kanäle sind, als Dopplergeschwindigkeiten interpretieren. So fallen quasi als Nebenprodukte der Bestimmung des magnetischen Flusses einfache Geschwindigkeitskarten an. Es ist natürlich zu beachten, dass hierbei die Information aus dem gesamten Verlauf der Spektrallinie genutzt wird. Hierdurch werden eventuelle Informationen über die Abhängigkeit der Geschwindigkeiten von der Höhe in der Sonnenatmosphäre verschmiert, es resultiert eine mittlere Geschwindigkeit des Plasmas aus einer „mittleren“ Höhenschicht, die am meisten zum Schwerpunkt des Linienprofils im Stokes-I-Signal beiträgt. Ein Geschwindigkeitsbild aus demselben Datensatz wie in Abb. 5.21 ist in Abb. 5.22 rechts gezeigt, im Vergleich zur Speckle-Rekonstruktion aus den CCD 1-Bildern. Die räumliche Auflösung beträgt auch hier ca. 0,4" - 0,5", maximale Geschwindigkeitswerte sind etwa $\pm 1,5 \mathrm{~km} / \mathrm{s}$. Eine genauere Bewertung der Daten erfolgt ebenfalls in Kap. 6.

Abschließend ist zu sagen, dass die Linienschwerpunktsmethode zwar unempfindlich auf 


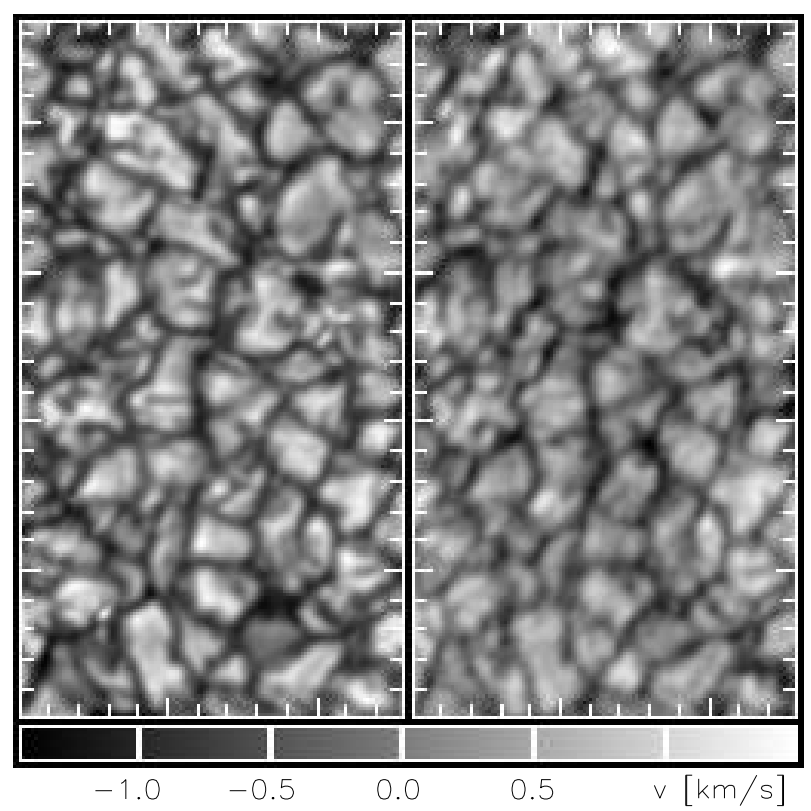

Abbildung 5.22: Rechts: Geschwindigkeitskarte, die für den Scan s5_4 aus den Linienschwerpunkten des Stokes-I-Signals berechnet wurde. Links: Zum Vergleich dazu die SpeckleRekonstruktion aus den CCD 1-Bildern. Der Abstand der Strichmarken entspricht 1".

viele störende Einflüsse reagiert, nicht jedoch auf Streulicht. Signal, das von einem unmagnetischen Gebiet in ein Bildelement mit einer magnetischen Struktur hineingestreut wird, kann die beiden Profile $\frac{1}{2}\left(I_{\lambda} \pm V_{\lambda}\right)$ verfälschen und mehr wie unmagnetische Profile erscheinen lassen. Dies gilt insbesondere dort, wo die eigentlichen magnetischen Strukturen dunkel sind und wenig eigenes Licht beitragen, z. B. in Poren. Das führt zu einer Unterschätzung des magnetischen Flusses in diesen Gebieten. Bei hinreichend starken Feldern in solchen Strukturen kann dann aber wieder auf die Größe der Aufspaltung des V-Signals zurückgegriffen werden, um die magnetischen Flussdichten zu bestimmen. Genaueres hierzu wird später in Kap. 6.2 besprochen. 


\section{Ergebnisse}

In diesem Kapitel sollen der Reihe nach die beobachteten Objekte gezeigt und die physikalischen Ergebnisse besprochen und in den sonnenphysikalischen Kontext eingebunden werden.

\subsection{Ruhige Granulation}

Die Scans s5_1 bis s5_4 vom 22.10.99 von einem nicht aktiven Granulationsgebiet sehr nahe der Scheibenmitte waren ursprünglich aufgenommen worden, um Daten zu Kalibrationszwecken zur Verfügung zu haben, die i. W. kein magnetisches Stokes-V-Signal zeigen. Doch schon diese Daten zeigen an vielen Stellen deutliche magnetische Strukturen, die im Folgenden genauer untersucht werden sollen.

Das Seeing war während der kurzen Zeitserie sehr gut, die Fried-Parameter waren $r_{0}=12,5 \mathrm{~cm}, 11,8 \mathrm{~cm}, 12,3 \mathrm{~cm}, 13,9 \mathrm{~cm}$, so dass sich in den Rekonstruktionen der CCD 2Bilder Strukturen bis hinunter zu 0,4" - 0,5" finden lassen. Ebensolche Auflösung findet sich in den Magnetfeld- und Geschwindigkeitskarten. In Abb. 6.1 sind für den Scan s5_4 mit dem besten Seeing die rekonstruierten Stokes-I-Bilder an den FPI-Positionen des Scans rund um den Linienkern der Fe I-Linie bei 630,25 nm abgebildet. In Abb. 6.2 sind zu allen vier Scans dieser Serie die Speckle-Rekonstruktionen der CCD 1-Bilder, die Magnetfeldkarten aus der Linienschwerpunktsmethode und die Geschwindigkeitsbilder gezeigt. All die Regionen, in denen sich Magnetfelder nachweisen lassen, sind für den Scan s5_4 durch Rechtecke markiert. Ähnliche Untersuchungen (u. a. Grossmann-Doerth et al. (1996), Sigwarth (1999)]) zeigten, dass noch größere Flächenanteile ruhiger Granulation schwache Magnetfelder aufweisen. Da diese Untersuchungen aber eine höhere magnetische Empfindlichkeit hatten, sind sie mit der in der vorliegenden Arbeit gefundenen Anzahl von magnetischen Strukturen konsistent. Einige der in Abb. 6.2 markierten Regionen sollen im Folgenden näher untersucht werden. Die in den Bildern angegebenen Flüsse sind jeweils auf ein Pixel bezogen $\left(72,5 \times 72,5 \mathrm{~km}^{2}\right.$ auf der Sonne). 


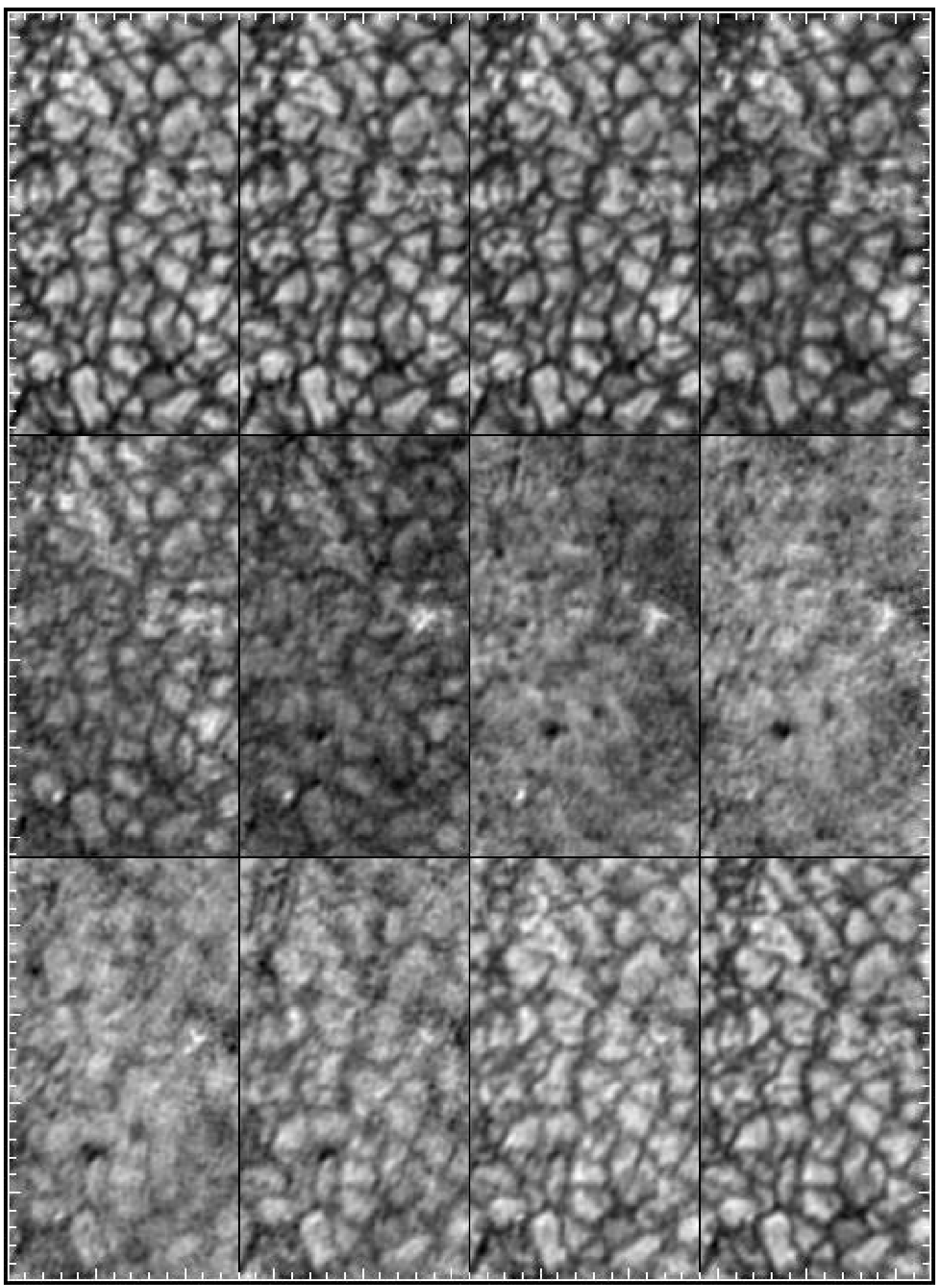

Abbildung 6.1: Von links oben nach rechts unten sind die rekonstruierten Stokes-I-Bilder des Scans s5_4 an den FPI-Positionen rund um den Linienkern der Fe I-Linie bei 630,25 nm abgebildet. Der Abstand der Strichmarken entspricht 1". 
s5-1

s5_2

s5_3

s5_4
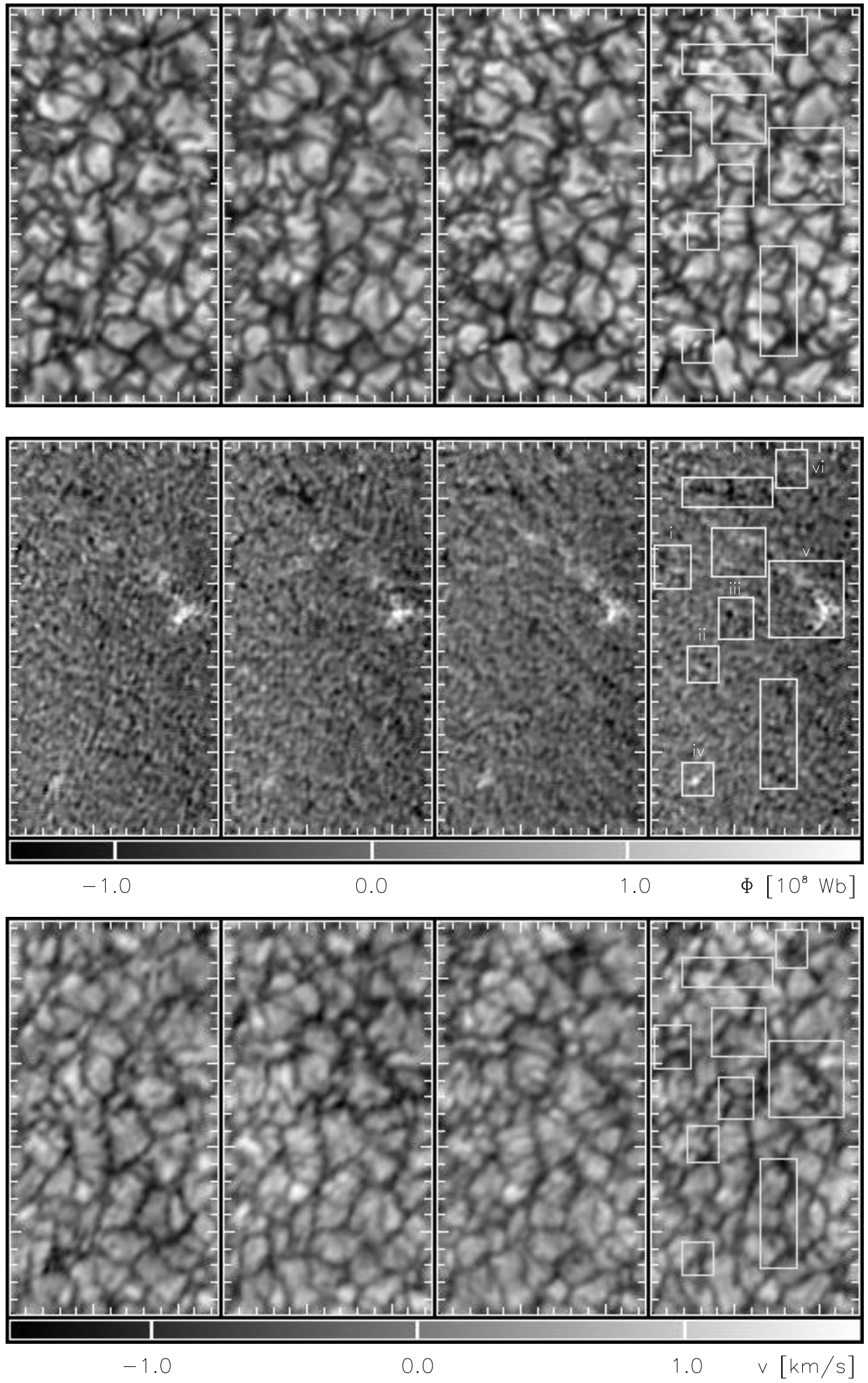

Abbildung 6.2: Für die Scans s5_1 bis s5_4 sind jeweils die Speckle-Rekonstruktionen der CCD 1-Bilder gezeigt (oben), die Karte des magnetischen Flusses (Mitte) und das Geschwindigkeitsbild (unten). Für s5_4 sind all die Regionen markiert, in denen sich Magnetfelder nachweisen lassen. Der Abstand der Strichmarken entspricht 1". 


\subsubsection{Gemischte Polaritäten innerhalb sehr kurzer Distanzen}

Die in Abb. 6.2 mit $i$ bis iii markierten Ausschnitte sind in den Abb. 6.3 bis 6.5 vergrößert dargestellt und in der Karte des magnetischen Flusses mit den über $3 \times 3$ Pixel gemittelten Stokes-V-Profilen überlegt. Sie sollen zunächst einmal die Empfindlichkeit der Messmethode zeigen und deutlich machen, welche schwachen Magnetfelder sich gerade eben noch detektieren lassen. In vielen Fällen sind sehr schwache, beinahe im Rauschen verborgene Stokes-V-Profile erkennbar, die aber jeweils den richtigen Bezug zu Stärke und Polarität der unterlegten Karte des magnetischen Flusses aus der Linienschwerpunktsmethode haben. Wichtig ist hier aber vor allen Dingen der direkte Nachweis, dass innerhalb sehr kurzer Distanzen (weniger als 1") magnetische Strukturen unterschiedlicher Polarität auftreten. Dies wurde bisher gemeinhin angenommen, es gab viele indirekte Hinweise dafür und theoretische Überlegungen, dass solche Strukturen existieren sollten. Hier nun sind die unterschiedlichen Polaritäten räumlich aufgelöst und direkt in Stokes-V-Profilen und den gemessenen magnetischen Flüssen aus der Linienschwerpunktsmethode sichtbar. Die geringsten, feststellbaren Abstände gegensätzlicher

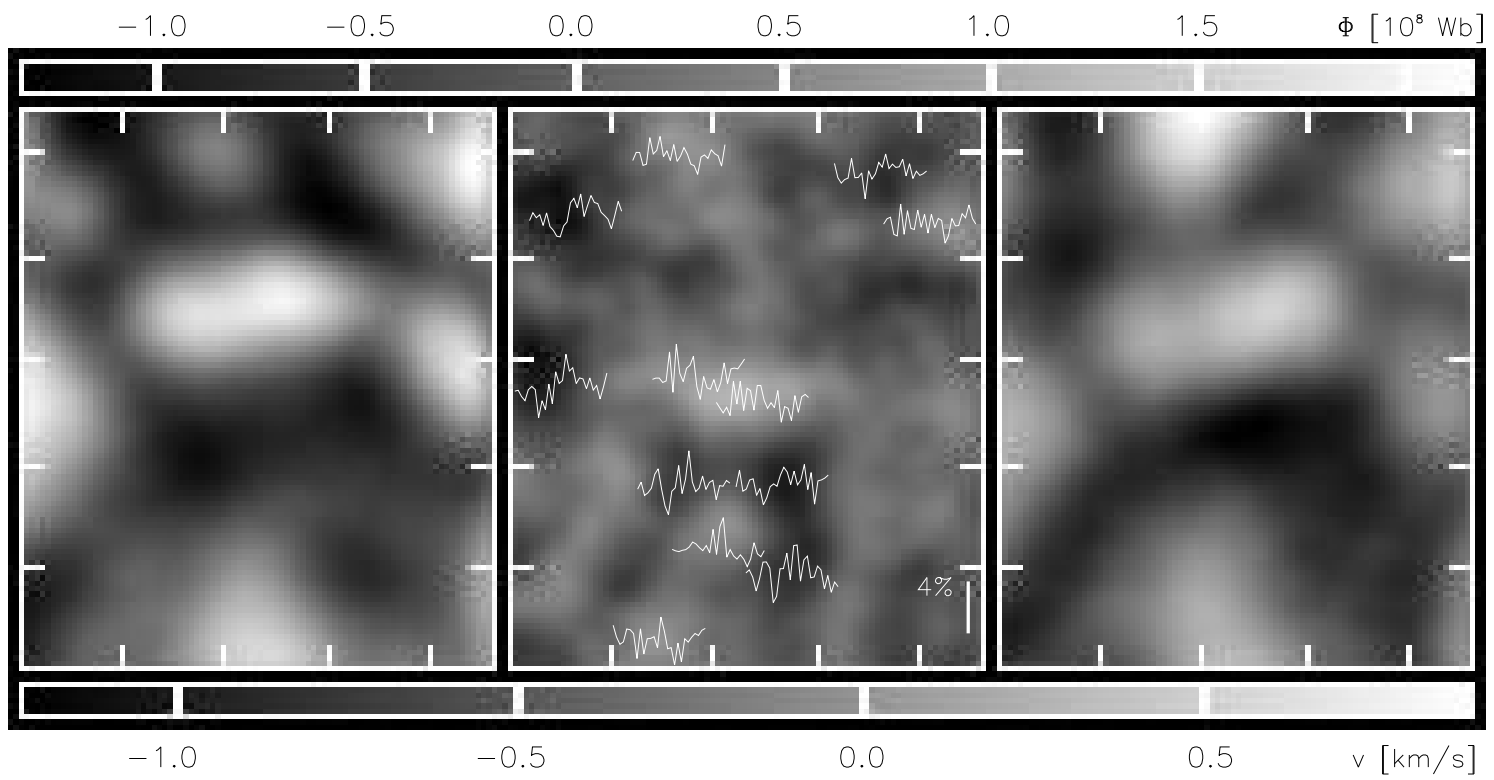

Abbildung 6.3: Ausschnitt aus s5_4, der in Abb. 6.2 mit i bezeichnet war. Gezeigt sind die Speckle-Rekonstruktion (links), die Karte des magnetischen Flusses, dem die über $3 \times 3$ Pixel gemittelten Stokes-V-Signale überlagert sind (Mitte) und das Geschwindigkeitsbild aus der Linienschwerpunktsmethode (rechts). Der Abstand der Strichmarken entspricht 0, $5^{\prime \prime}$.

Polaritäten sind 0,4". Dies sind z. B. in Abb. 6.3 das in der magnetischen Flusskarte hell dargestellte magnetische Elemente in der Bildmitte (positive Polarität) und das dunkel dargestellte Element direkt darunter (negative Polarität). Beide zeigen, zwar gerade am Detektionslimit, aber doch sichtbar, auch in ihrem Stokes-V-Profil die Signatur der entsprechenden Magnetfelder. Sie liegen am Rande einer schwach aufströmenden Granule 


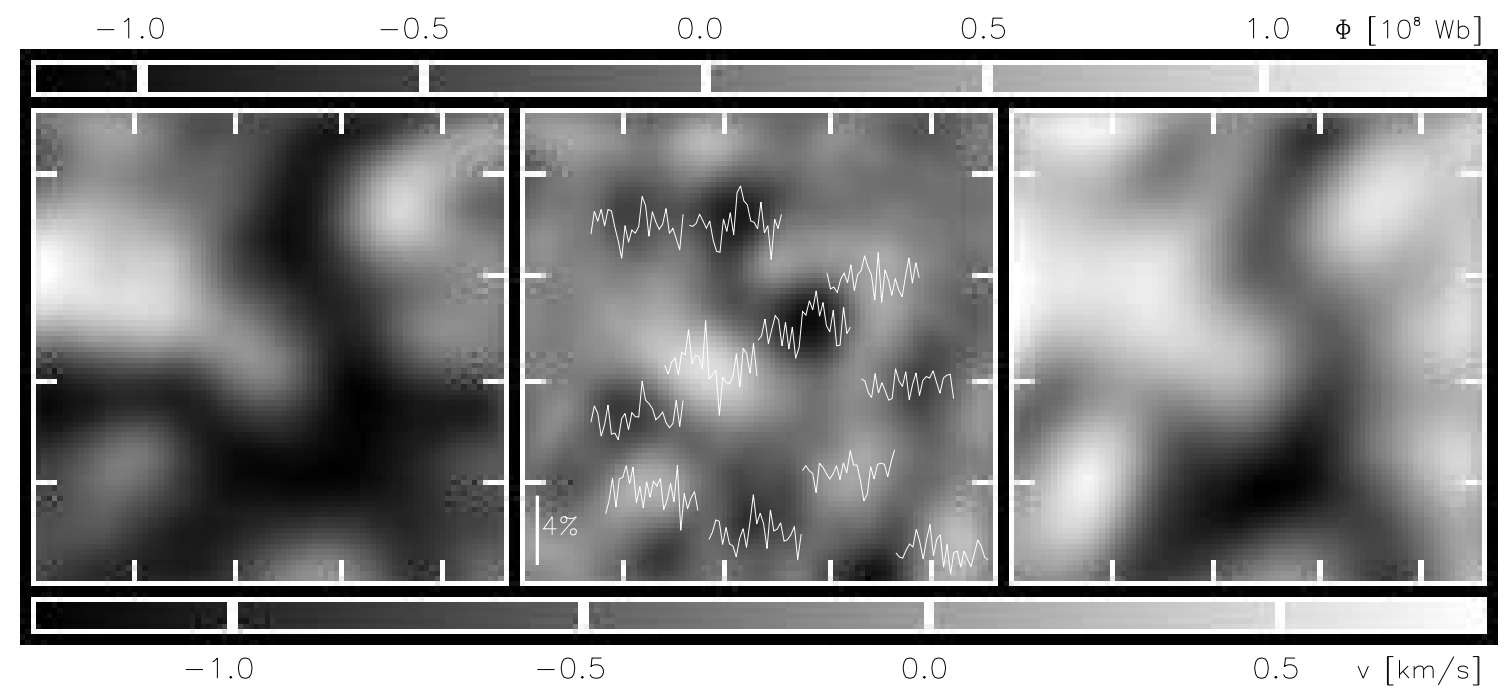

Abbildung 6.4: Dasselbe wie in Abb. 6.3, aber für den Ausschnitt ii. Der Abstand der Strichmarken entspricht $0,5^{\prime \prime}$.

in einem intergranularen Raum, der an dieser Stelle Abströmungen von 0,5 bis 1,2 km/s aufweist. Die maximalen magnetischen Flüsse durch ein Pixel sind $\pm 1,2 \cdot 10^{8} \mathrm{~Wb}$ in diesen beiden Strukturen.

Ähnliche Verhältnisse finden sich in Abb. 6.4. In einem typischen Abstand von $0,5^{\prime \prime}$ treten mehrfach wechselnde Polaritäten auf, deren maximale magnetische Flüsse $\pm 1,1 \cdot 10^{8} \mathrm{~Wb}$ in einem Pixel betragen. Neben mehreren magnetischen Strukturen in abströmenden Intergranulen tritt hier u. a. bei dem hell dargestellten Element nahe der Bildmitte eine Struktur auf, die sich, im Grenzgebiet zwischen einer Granule und einer Intergranule, in einer Region schwacher Aufströmung von 0,1-0,4 km/s befindet. Das Element negativer Polarität am unteren Bildrand in der Mitte befindet sich dagegen in einem breiten, intergranularen Raum und zeigt eine starke Abströmung von 1,3 km/s.

In Abb. 6.5 schließlich findet sich eine kräftigere magnetische Struktur mit einem Fluss von $1,9 \cdot 10^{8} \mathrm{~Wb}$ in einem Pixel negativer Polarität im Intergranulum mit schwacher Abströmung von $0,1-0,5 \mathrm{~km} / \mathrm{s}$. Im Abstand von 0,5" rechts davon und darüber befinden sich zwei schwache Strukturen positiver Polarität mit Flüssen von $0,8 \cdot 10^{8} \mathrm{~Wb}$ in einem Pixel am Rande einer großen, sich zerteilenden Granule. Diese, wie auch die meisten weiteren Strukturen in diesem Bildausschnitt, zeigen moderate Auf- oder Abströmungen von wenigen hundert $\mathrm{m} / \mathrm{s}$. Lediglich die Struktur unten links liegt in einer starken Abströmung von $1,3 \mathrm{~km} / \mathrm{s}$.

Bei genauer Inspektion der Daten finden sich viele weitere Stellen in der ruhigen Granulation, an denen sich schwache Felder von $0,5-1,0 \cdot 10^{8} \mathrm{~Wb}$ in einem Pixel nachweisen lassen, die häufig innerhalb von sehr kurzen Distanzen von weniger als 1" Polaritätswechsel zeigen. Häufig, aber nicht immer, liegen diese in intergranularen Räumen und zeigen ein breites Spektrum von Geschwindigkeiten, nämlich von schwachen Aufströmungen (einige hundert $\mathrm{m} / \mathrm{s}$ ) bis zu starken Abströmungen (bis zu 1,3 km/s). 


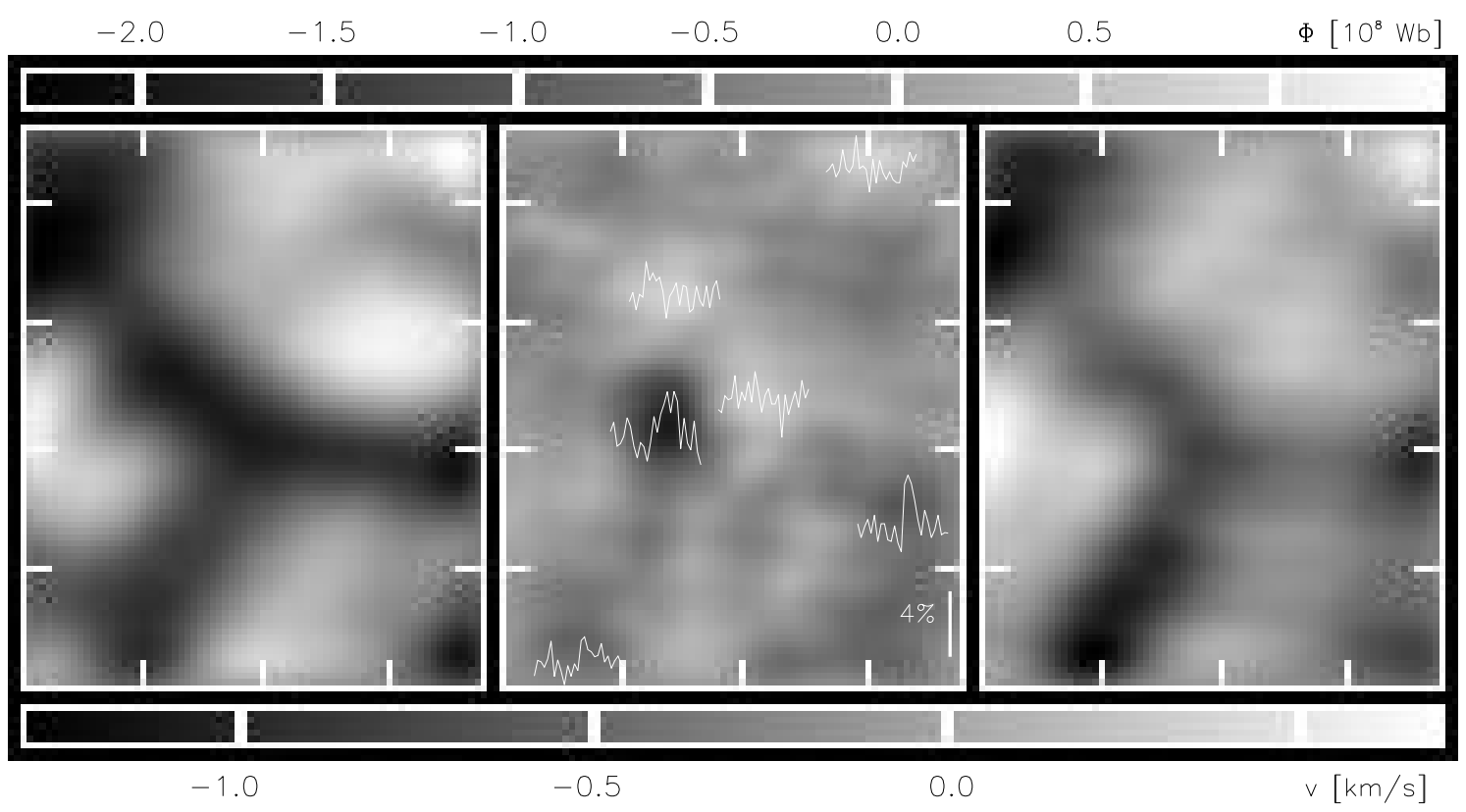

Abbildung 6.5: Dasselbe wie in Abb. 6.3, aber für den Ausschnitt iii. Der Abstand der Strichmarken entspricht $0,5^{\prime \prime}$.

Dieses Ergebnis steht im Gegensatz zu der Untersuchung von Keller \& v. d. Lühe (1992), die mit derselben Bildrekonstruktionsmethode eine räumliche Auflösung von unter 1" erreicht hatten und dabei keine gemischten Polaritäten innerhalb kurzer Distanzen nachweisen konnten. Allerdings lagen bei ihnen keine vollen Stokes-V-Profile vor, sondern relativ breitbandige Aufnahmen im Linienflügel der magnetisch sensitiven Fe I-Linie bei $525,0 \mathrm{~nm}$. Außerdem untersuchten sie eine unipolare, aktive Region, während sich das vorliegende Ergebnis auf sehr schwache Felder in ansonsten nicht-aktiver Granulation bezieht.

\subsubsection{Verstärkung von magnetischen Strukturen durch konvektiven Kollaps}

In Abb. 6.6 ist eine kurze Zeitserie des Ausschnitts iv gezeigt. Der Zeitabstand zwischen den Zeilen beträgt 74s, wieder ist links die Speckle-Rekonstruktion im Breitbandkanal gezeigt, in der Mitte die Karte des magnetischen Flusses mit V-Profilen und rechts das Geschwindigkeitsbild. Die Skalierungen sind für alle Datensätze gleich gewählt.

$\mathrm{Zu}$ Beginn der Zeitserie findet sich ein intergranularer Zwischenraum mit sehr starken Abströmungen von bis zu 1,4km/s. In diesem befinden sich mehrere kleine magnetische Strukturen beider Polaritäten. Am stärksten ist das Element unten links mit negativer Polarität und einem Fluss von $1,8 \cdot 10^{8} \mathrm{~Wb}$ in einem Pixel. In den folgenden Scans ist zu beobachten, dass zwei expandierende Granulen mit Aufströmungen von bis zu 


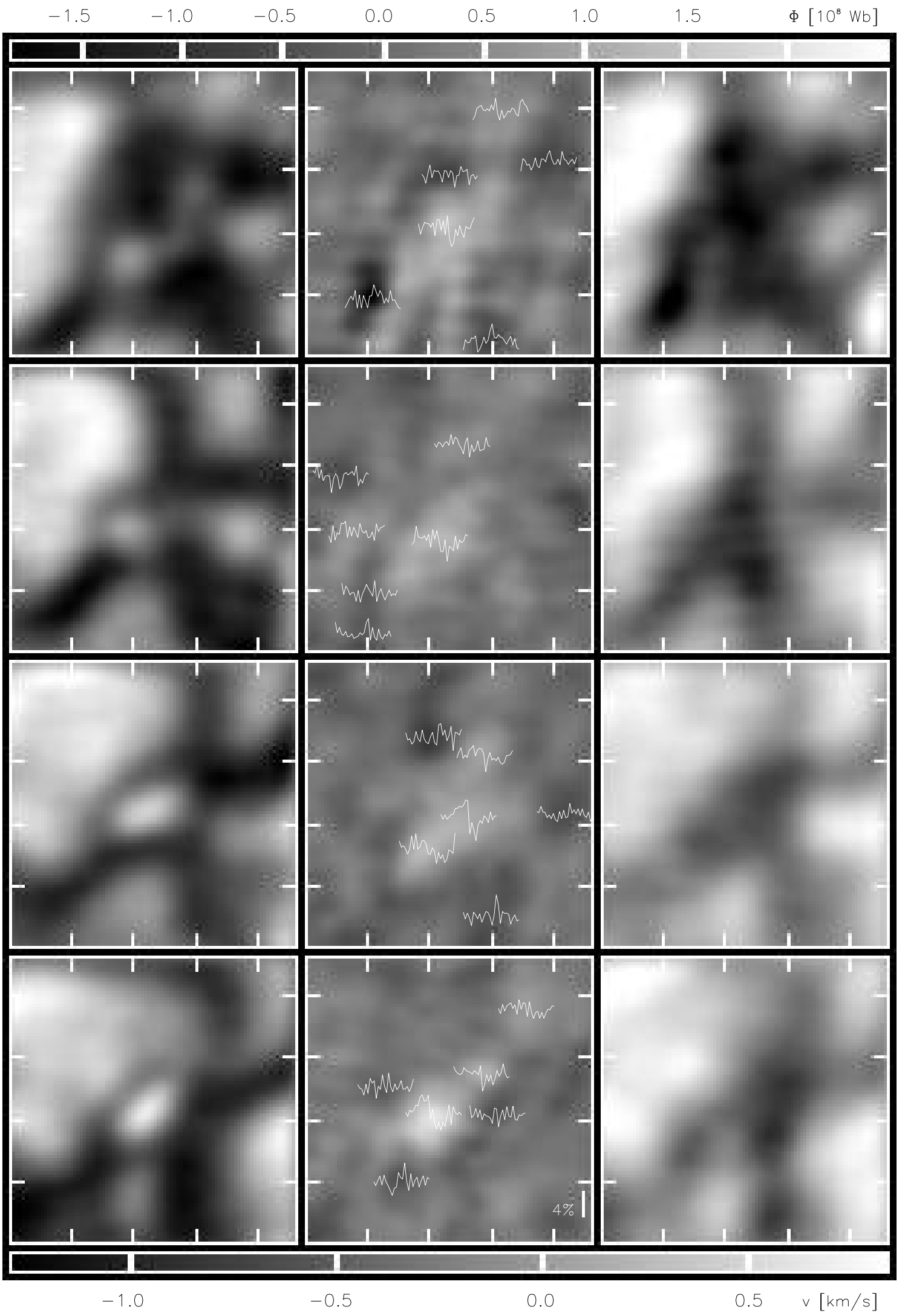

Abbildung 6.6: Zeitserie des Ausschnitts iv in Scan s5_4. Der Abstand der Strichmarken entspricht $0,5^{\prime \prime}$. 


\subsection{Ruhige Granulation}

$1 \mathrm{~km} / \mathrm{s}$ von links und von unten rechts diesen Zwischenraum zusammendrücken, dabei geht die Abströmung auf kleinere räumliche Gebiete und Werte um $1 \mathrm{~km} / \mathrm{s}$ zurück. Gleichzeitig bildet sich im Bild der Kontinuumsintensität eine kleine, starke Aufhellung, ein Fackelpunkt, der besonders in den Linienkernbildern deutlich zu sehen ist, vgl. auch Abb. 6.1 und 6.2. Die Anordnung der magnetischen Strukturen in diesem Bereich verändert sich. Das erwähnte, negative Element verschwindet fast vollständig, dafür wird das zunächst etwas ausgedehntere Element positiver Polarität in der Bildmitte konzentriert, sein Flächenanteil wird kleiner, dafür steigt der magnetische Fluss in der Mitte des Elements von ca. 1, 5 $10^{8} \mathrm{~Wb}$ auf $2,1 \cdot 10^{8} \mathrm{~Wb}$ in einem Pixel. Der Gesamtfluss in diesem Element bleibt während der Entwicklung ungefähr gleich hoch bei einem Wert von $5 \cdot 10^{10} \mathrm{~Wb}$. Zwar befindet sich im letzten Scan der Ort stärkster Abströmung ca. 0,3" neben dem Zentrum der konzentrierten, magnetischen Struktur, trotzdem besteht die Möglichkeit, dass an dieser Stelle die schon in Kap. 2.1 beschriebene Entstehung starker Flussröhren durch konvektiven Kollaps beobachtet wurde.

\subsubsection{Zusammenhang zwischen magnetischem Fluss und Intensität}

Bei genauer Betrachtung der bisher besprochenen Bildausschnitte gewinnt man leicht den Eindruck, dass kleine magnetische Strukturen innerhalb ansonsten ruhiger Granulation prinzipiell zwar an vielen möglichen Stellen auftreten können, bevorzugt aber in Intergranulen oder im Grenzbereich zwischen Granulen und Intergranulen zu finden sind. Dort sind sie manchmal durch kleinskalige Aufhellungen (Filigree) markiert, häufig aber auch dunkel und völlig unauffällig. Weiterhin stellt man z. B. in Abb. 6.1 fest, dass sich magnetische Strukturen in Linienkernbildern deutlich durch erhöhte Intensität abheben, worauf es bereits in der Literatur viele Hinweise gibt.

Eine statistische Untersuchung soll die Frage beantworten, ob die Verteilung der Intensitäten (sowohl im Kontinuum wie auch im Linienkern) magnetischer Strukturen von der Stärke der Magnetfelder abhängt und ob sich diese Verteilung von der der unmagnetischen Granulation unterscheidet. Dazu wurden aus den vier vorliegenden GranulationsScans Rechtecke herausgeschnitten, die magnetische Strukturen enthalten, i. W. sind dies die in Abb. 6.2 markierten Ausschnitte. Dann wurde Pixel für Pixel die Intensität gegen den magnetischen Fluss als Punktwolke aufgetragen. Für die Kontinuumsintensitäten wurden die Speckle-Rekonstruktionen der CCD 1-Bilder verwendet, für die Linienkernintensitäten die entsprechenden Positionen aus den schmalbandigen CCD 2-Datenblöcken. Das Ergebnis ist in Abb. 6.7 gezeigt. Die Messpunkte, die magnetische Flüsse unterhalb des Detektionslimits von $0,5 \cdot 10^{8} \mathrm{~Wb}$ in einem Pixel zeigen, können als Referenzwerte unmagnetischer Granulation angesehen werden. Strukturen mit schwachen Magnetfeldern zwischen 0,5 und $1,0 \cdot 10^{8} \mathrm{~Wb}$ durch ein Pixel zeigen eine leichte Unterrepräsentation von Kontinuumsintensitäten, die über der mittleren Intensität $\bar{I}_{\text {Kont }}$ liegen. Diese Strukturen treten also tatsächlich bevorzugt in intergranularen Räumen auf. Gleiches gilt für Felder zwischen 1,0 und $1,5 \cdot 10^{8} \mathrm{~Wb}$ in einem Pixel. Noch stärkere Felder oberhalb $1,5 \cdot 10^{8} \mathrm{~Wb}$ 


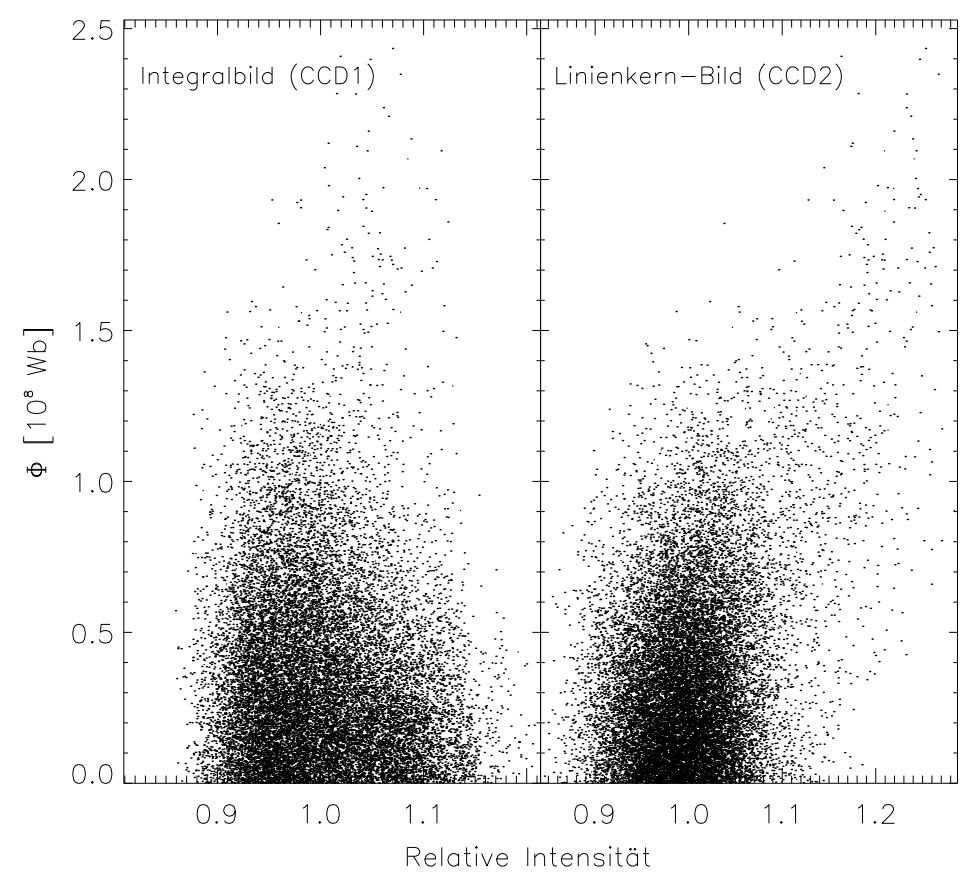

Abbildung 6.7: Zusammenhang zwischen magnetischem Fluss in einem Pixel und Intensität für einzelne Pixel der Granulationsscans s5_1 bis s5_4. Links sind die Intensitäten in der Speckle-Rekonstruktion benutzt, rechts die im Linienkernbild.

in einem Pixel zeigen dagegen in der Regel leicht überdurchschnittliche Intensitäten von bis zu $1,13 \cdot \bar{I}_{\text {Kont }}$. Alle die Pixel mit den größten Intensitäten von mehr als $1,15 \cdot \bar{I}_{\text {Kont }}$ stammen aus dem Innern „normaler“, ungestörter Granulen und zeigen keine merklichen Magnetfelder. Ganz anders verhält es sich bei der Linienkernintensität: Hier gibt es einen monotonen Zusammenhang zwischen magnetischem Fluss und Intensität. Die feldfreien Stellen zeigen fast ausnahmslos moderate Intensitäten von maximal $1,15 \cdot \bar{I}_{\text {Kern }}$, dagegen zeichnen sich alle Magnetfelder stärker als $1,5 \cdot 10^{8} \mathrm{~Wb}$ in einem Pixel durch deutliche Aufhellungen bis zu $1,3 \cdot \bar{I}_{\text {Kern }}$ aus.

In Abb. 6.8 sind nochmals für je $0,5 \cdot 10^{8} \mathrm{~Wb}$ breite Intervalle die Häufigkeitsverteilungen der Kontinuums- (durchgezogen) und Linienkernintensitäten (gepunktet) gezeigt. In der 2. und 3. Auftragung ist wieder deutlich zu erkennen, dass die Verschiebung der Verteilung zu dunkleren Strukturen im Kontinuum in erster Linie durch das Fehlen von Magnetfeldern in den helleren Granulen zustande kommt. Die unterste Auftragung zeigt die Abhängigkeit der mittleren relativen Intensität für Strukturen mit gegebenem magnetischen Fluss. Beide Abbildungen zeigen, was zusammenfassend gesagt werden kann: Schwache magnetische Strukturen (weniger als $1,5 \cdot 10^{8} \mathrm{~Wb}$ in einem Pixel) sind im Kontinuum meistens dunkel und befinden sich bevorzugt in intergranularen Räumen, stärkere Magnetfelder machen sich häufiger als helles Filigree bemerkbar. Anders im Li- 

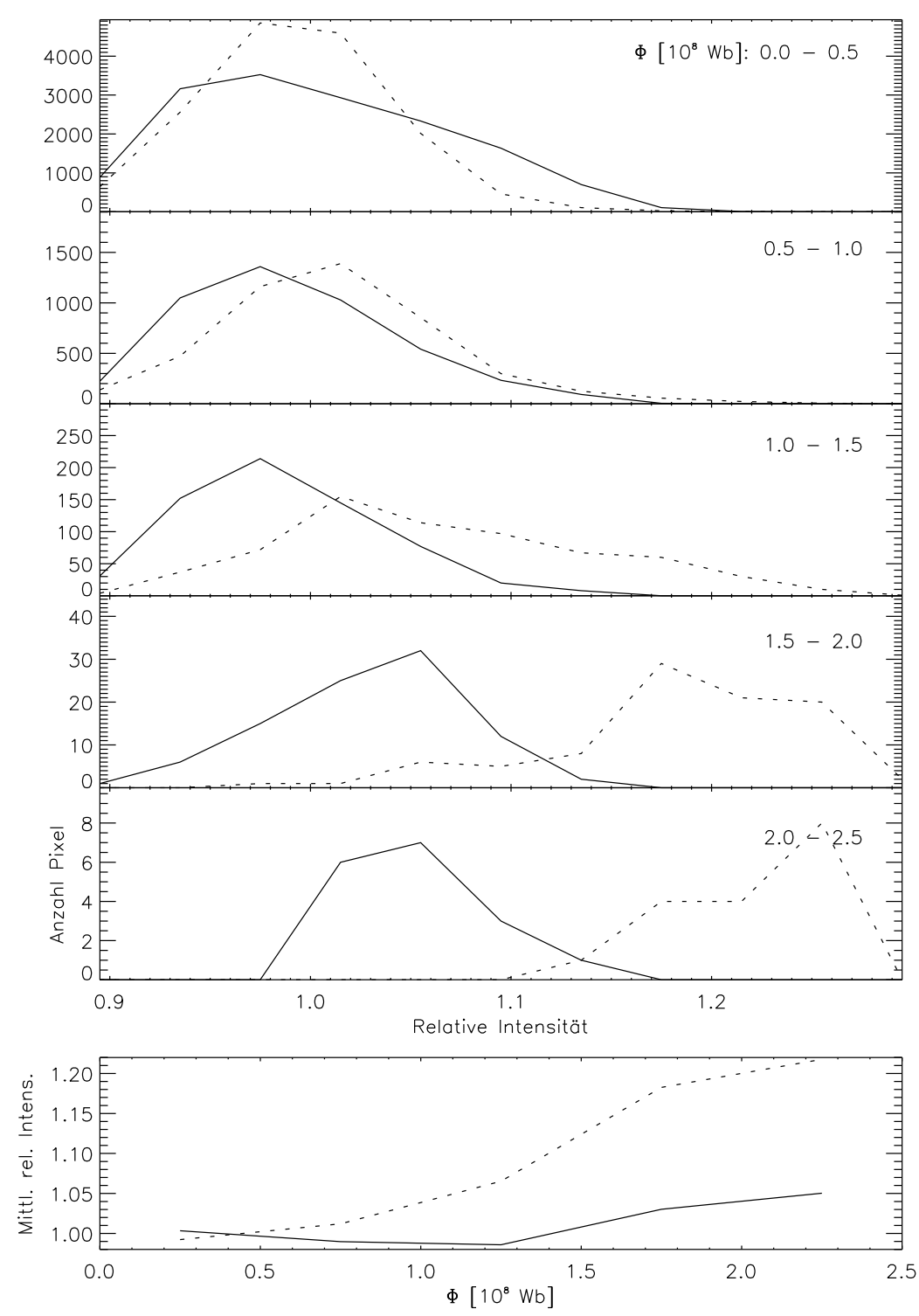

Abbildung 6.8: Histogramme der Intensitätsverteilung für verschiedene magnetische Flüsse durch ein Pixel. Durchgezogen sind jeweils die Häufigkeiten von Pixeln bestimmter Kontinuumsintensität aufgetragen, gepunktet für die Linienkernintensitäten, von oben nach unten für steigenden magnetischen Fluss. In der untersten Auftragung sind die gemittelten Intensitäten für die obigen Fluss-Bereiche gezeigt.

nienkern, dort zeichnen sich magnetische Strukturen fast immer durch erhöhte Intensität aus, und je höher der magnetische Fluss ist, desto höher ist auch die Linienkernintensität. Ursache des letzteren ist, dass das Licht des Linienkerns höheren Atmosphärenschichten entstammt, in denen das Material oberhalb magnetischer Strukturen aufgeheizt ist.

Insgesamt gingen 20000 Pixel aus den vier Granulations-Scans in diese Untersuchung ein. 


\subsubsection{Zusammenhang zwischen magnetischem Fluss und Strömungen}

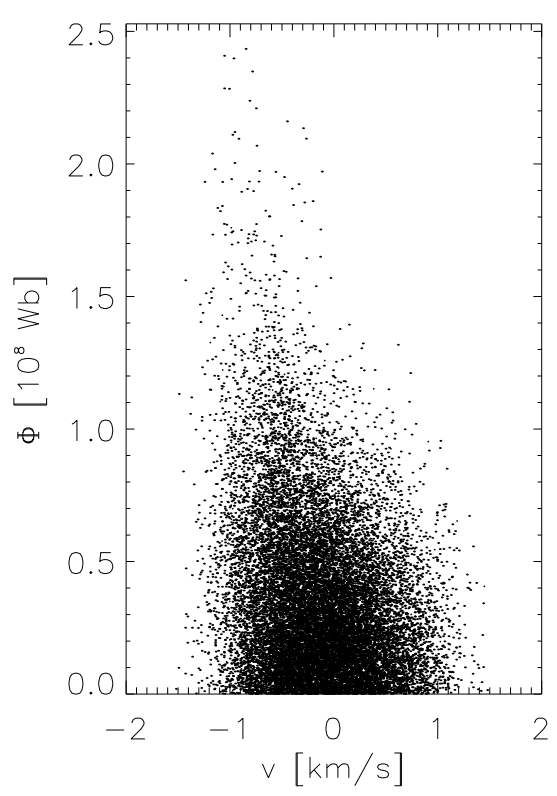

Abbildung 6.9: Zusammenhang zwischen magnetischem Fluss und Geschwindigkeiten für einzelne Pixel der Granulationsscans s5_1 bis s5_4.
Auf ähnliche Weise kann untersucht werden, ob magnetische Strukturen bestimmter Stärke bevorzugt mit Auf- oder Abströmungen zusammentreffen. Abb. 6.9 zeigt, dass mit stärkerem Magnetfeld immer weniger Aufströmungen vorkommen. Die Werte der Abströmungen liegen in derselben Größenordnung von $1-1,5 \mathrm{~km} / \mathrm{s}$ wie in den unmagnetischen Intergranulen. Hierbei ist allerdings zu berücksichtigen, dass diese Werte, die mit der Linienschwerpunktsmethode bestimmt wurden, einen mittleren Geschwindigkeitswert für das gesamte Flächenelement eines Pixels und den gesamten von der Spektrallinie überdeckten Höhenbereich darstellen. Es ist deshalb durchaus denkbar, dass in den Flussröhren selbst, deren Füllfaktor vermutlich deutlich kleiner als eins ist, erheblich höhere Geschwindigkeiten auftreten. Hier könnte die „zero crossing“Geschwindigkeit (vgl. Kap. 5.2.3) Aufschluss geben. Leider sind jedoch die Stokes-V-Signale für Magnetfelder dieser Größenordnung so verrauscht, dass eine genaue Bestimmung des Nulldurchgangs nicht möglich ist.

Abb. 6.10 zeigt wiederum für Bereiche verschieden starker Magnetfelder die Häufigkeitsverteilung der auftretenden Strömungen sowie in der untersten Auftragung die mittlere Strömungsgeschwindigkeit für diese Bereiche. Bei schwachen bis mittleren Magnetfeldern zwischen 0,5 und 2,0 $\cdot 10^{8} \mathrm{~Wb}$ in einem Pixel treten im Mittel schwache Abströmungen von einigen $100 \mathrm{~m} / \mathrm{s}$ auf.

\subsubsection{Dynamik und Lebensdauer kleiner Strukturen}

Die Dynamik, Wechselwirkung und die Lebensdauer schwacher magnetischer Elemente in der Granulation ist sehr variabel. Ein Beispiel von Fluss-Konzentration und Bildung eines deutlichen, hellen Fackelpunkts wurde bereits weiter oben beschrieben (vgl. Abb. 6.6). In Abb. 6.11 soll die Entwicklung der stärksten magnetischen Struktur im Beobachtungsgebiet (der Ausschnitt v aus Abb. 6.2) beschrieben werden.

Wieder befindet sich die Struktur im Intergranulum zwischen mehreren, teilweise zerplatzenden Granulen. Starke Abströmungen von bis zu 1,4 km/s prägen das Intergranulum, 

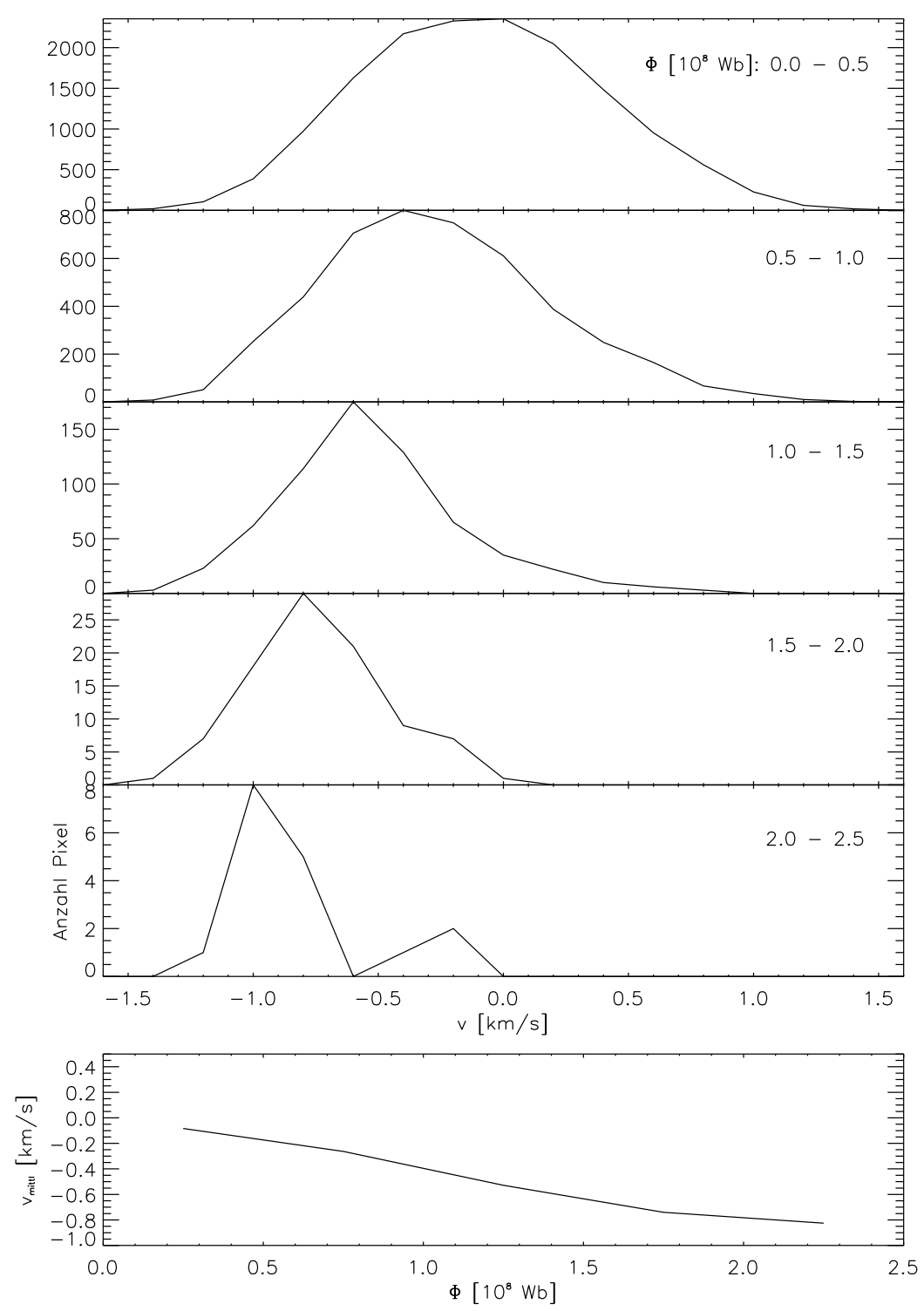

Abbildung 6.10: Histogramme der Geschwindigkeitsverteilung für verschiedene magnetische Flüsse. In der untersten Auftragung sind die gemittelten Geschwindigkeiten für die obigen Fluss-Bereiche gezeigt.

und hier finden sich auch jeweils die stärksten Magnetfelder mit bis zu 2,5 $\cdot 10^{8} \mathrm{~Wb}$ in einem Pixel und einer Stärke des Stokes-V-Signals von 3,5\%. Gemäß Abb. 4.3 lässt sich daraus für einen Plattenabstand von FPI 2 von $1 \mathrm{~mm}$ ein wahres V-Signal von $7 \%$ bei optimaler spektraler Auflösung abschätzen. Dies passt gut zu Messungen von Sigwarth (1999) des magnetischen Netzwerks, der ebenfalls in der Fe I-Linie bei 630,25 nm in magnetischen Knoten Polarisationsgrade von $8 \%$ findet, allerdings bei einer räumlichen Auflösung von 0,8-1". Auffällig ist bei dieser etwas stärkeren Struktur, dass wenig gemischte Polaritäten auftreten, lediglich einige kleine Strukturen negativer Polarität sind 


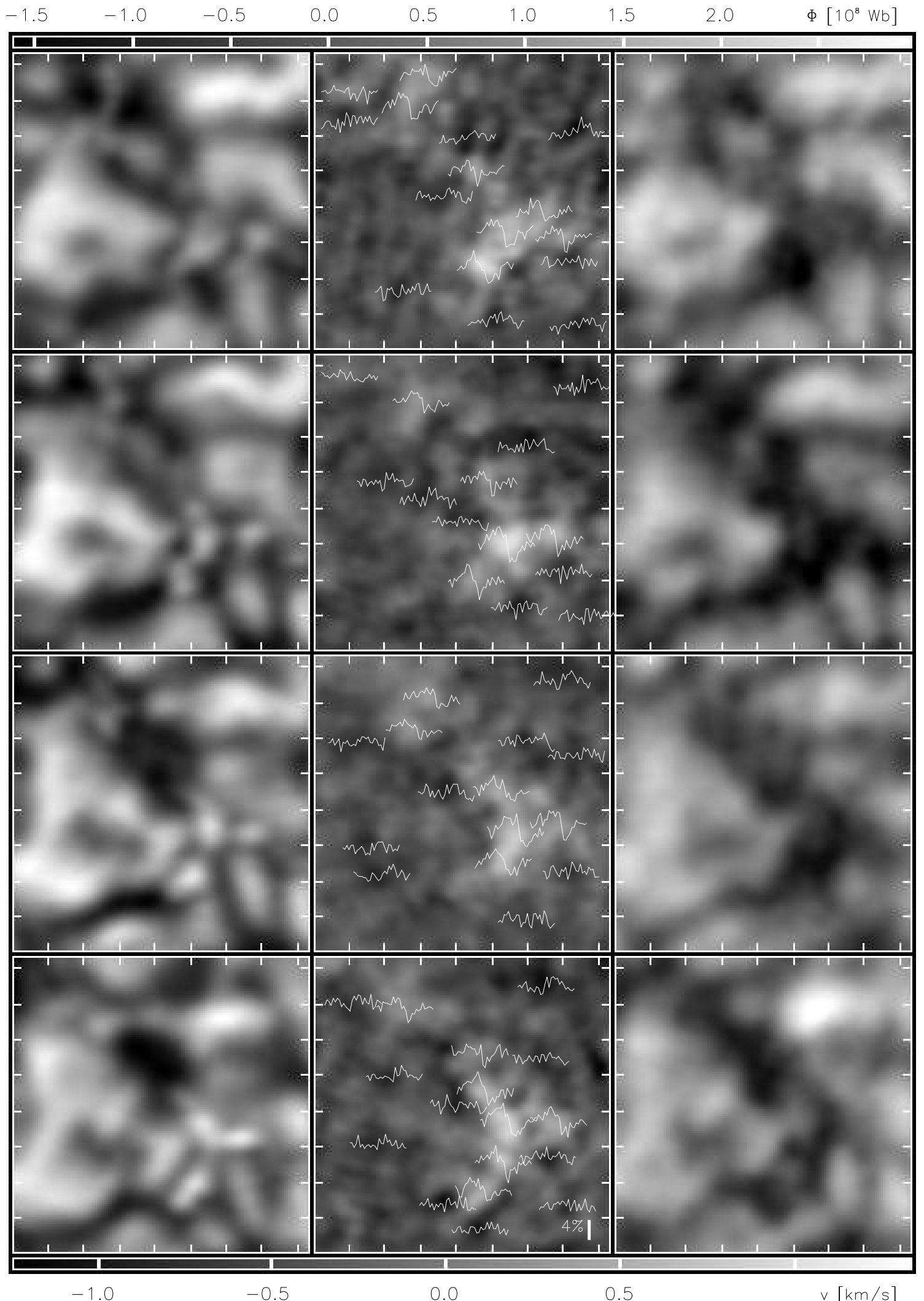

Abbildung 6.11: Zeitserie des Ausschnitts v in Scan s5_4. Links: Speckle-Rekonstruktion, Mitte: Magnetkarte, rechts: Geschwindigkeiten. Der Abstand der Strichmarken entspricht 0,5". 


\subsection{Ruhige Granulation}

im Linienschwerpunktsbild sichtbar. Nur bei einigen davon hebt sich das Magnetfeld auch gerade eben im Stokes-V-Signal ab, z. B. beim dritten Zeitschritt links neben der Bildmitte. Diese kleinsten, schwachen Magnetfelder lassen sich teilweise über mehrere Zeitschritte verfolgen, teilweise entstehen oder verschwinden sie aber auch während der kurzen Zeitserie oder verschieben, spalten oder vereinigen sich.

Die positive, etwas kräftigere Struktur verändert sich ebenfalls während der knapp fünf Minuten. Während die grobe Form und die Anordnung der einzelnen Strukturgruppen in Form eines über ca. $7^{\prime \prime}$ reichenden Bogens ungefähr erhalten bleibt (vgl. Abb. 6.2), unterscheiden sich die einzelnen kleinen Elemente von Zeitschritt zu Zeitschritt. Parallel dazu ändert sich das Aussehen im Kontinuum, hier entstehen aus einem "Gewirr" kleiner, undeutlicher Strukturen ab dem zweiten Zeitschritt drei sich immer deutlicher abhebende, helle Fackelpunkte, in denen auch der Hauptteil des Flusses konzentriert ist. Die Fackelpunkte werden scheinbar von der umliegenden Granulation hin- und hergeschoben. Durch die Intrusion des Materials aus der zerplatzenden Granule links von den Fackelpunkten wird die magnetische Struktur zu einem kleinen Bogen verformt und komprimiert. Ein kleines, aufströmendes Bruchstück dieser Granule könnte im SpeckleBild gerade selbst für einen Fackelpunkt gehalten werden, die Magnetkarte zeigt aber deutlich, dass es sich um ein feldfreies Granulenfragment handelt. Insgesamt ist fast die gesamte Granule feldfrei, lediglich in dem für zerplatzende Granulen typischen, dunklen „Loch“, in dem in der Mitte der Granule schon wieder kühleres Material absinkt, ist ein sehr schwaches, negatives Magnetfeld nachweisbar.

Die hellen Fackelpunkte zeigen hier die relativ stärksten Magnetfelder. Wie schon in den bisher beschriebenen Bildausschnitten findet man aber auch in diesem Bildausschnitt z. T. deutliche Magnetfelder am Rande oder zwischen den Granulen, die keine Aufhellungen zeigen oder gar ausgesprochen dunkel sind, wie z. B. die Struktur mit positiver Signatur am oberen Bildrand des ersten Zeitschritts.

Insgesamt scheint die Dynamik der magnetischen Strukturen i. W. durch die Dynamik der umliegenden Granulation bestimmt zu sein, und das um so mehr, je schwächer die Magnetfelder sind. Gerade die schwächsten Strukturen zeigen auch den schnellsten Wechsel von Positionen und die kürzesten Lebensdauern. Man vergleiche hierzu auch nochmals die Abb. 6.6. Teilweise kann bei den Zeitschritten von $74 \mathrm{~s}$ nicht klar entschieden werden, welche Strukturen in aufeinanderfolgenden Aufnahmen identisch sind. Zu beachten ist hier allerdings, dass so nahe am Detektionslimit Strukturen auch im Rauschen oder im Seeing untergehen oder in Form und Lage leicht verändert werden können, was auch durch die Bildrekonstruktion nicht völlig korrigiert werden kann.

Bemerkenswert ist in diesem Zusammenhang die Struktur im Ausschnitt vi aus Abb. 6.2, die als Zeitserie in Abb. 6.12 dargestellt ist. In den Zeitschritten 1, 3 und 4 treten nur sehr schwache, magnetische Strukturen auf, mit wechselnden Polaritäten und Positionen, die Flüsse betragen typischerweise $0,5-1,0 \cdot 10^{8} \mathrm{~Wb}$ in einem Pixel und sind im Stokes-V-Profil teilweise kaum nachweisbar. Im 2. Zeitschritt tritt allerdings kurzfristig 


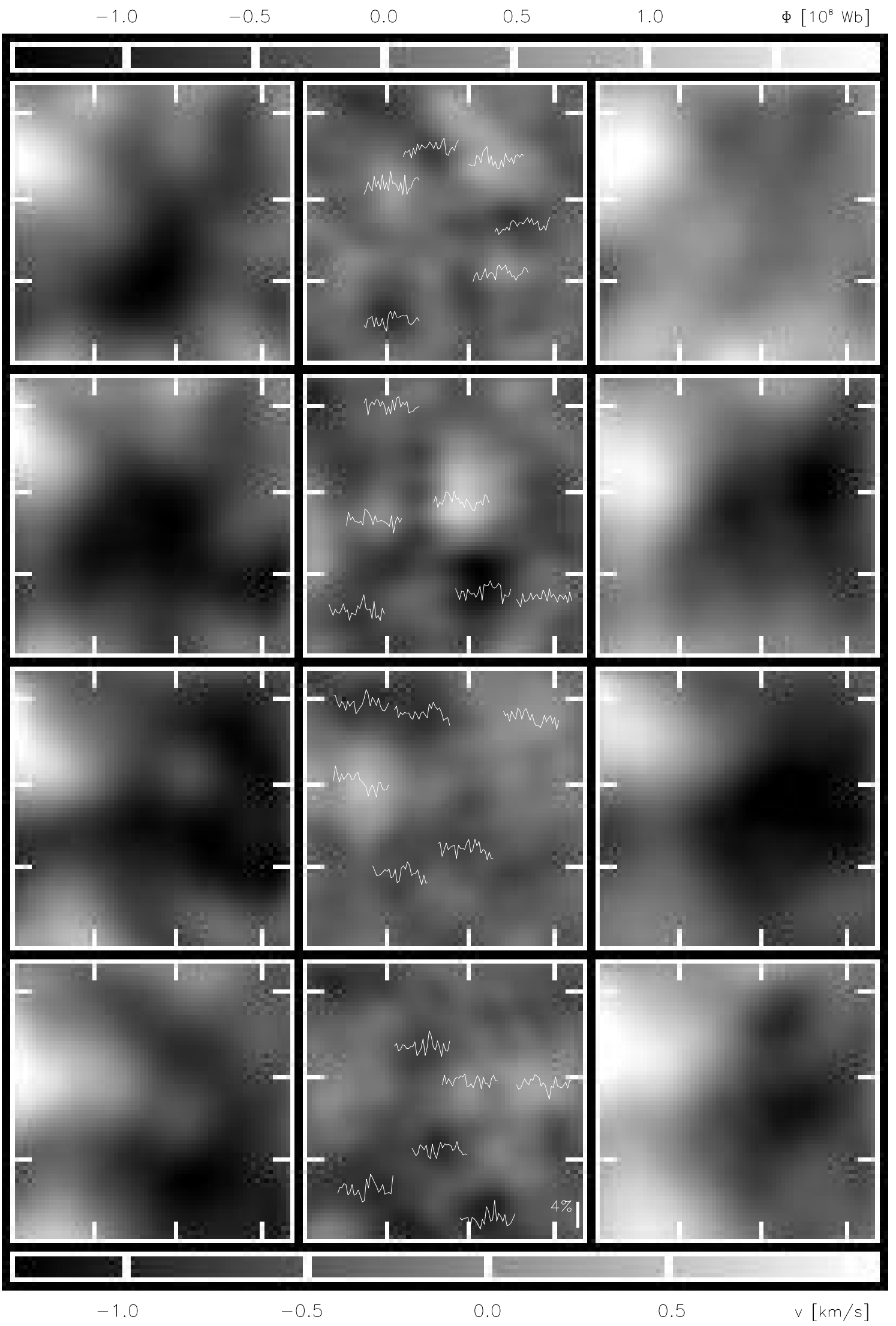

Abbildung 6.12: Zeitserie des Ausschnitts vi in Scan s5_4, sonst wie Abb. 6.11. Der Abstand der Strichmarken entspricht $0,5^{\prime \prime}$. 
eine etwas stärkere bipolare Struktur auf, deren Elemente im Abstand von 0,5" einen Fluss von $\pm 1,5 \cdot 10^{8} \mathrm{~Wb}$ in einem Pixel und ein (um das Aufösungsvermögen des FPIs bereinigtes) V-Signal von 3\% aufweisen. Gleichzeitig setzt in diesem ca. 1 Quadratbogensekunde großen Bereich recht plötzlich eine starke Abströmung von 1,5 km/s ein, die schon im 4. Zeitschritt wieder deutlich abklingt. In der Speckle-Rekonstruktion ist in diesem Bereich bis auf ein großes, etwas strukturiertes Intergranulum im Eckbereich mehrerer Granulen kein Anzeichen von Aktivität zu erkennen.

\subsubsection{Größe und magnetischer Fluss typischer Strukturen}

Anhand der vorliegenden Beobachtungsergebnisse lassen sich obere Grenzen für die Größe magnetischer Strukturen resp. Flussröhren angeben. Viele indirekte Hinweise und theoretische Modelle lassen erwarten, dass die wahren Durchmesser der Flussröhren erheblich kleiner sind als die bisher spektroskopisch erzielte räumliche Auflösung von ca. $1^{\prime \prime}$ ( $\widehat{=} 725 \mathrm{~km}$ auf der Sonne). Für die hier beobachteten magnetischen Strukturen sind häufig Durchmesser von $0,4^{\prime \prime}-0,5^{\prime \prime}$ bei ungefähr kreisförmig symmetrischen Formen festzustellen, z. B. in Abb. 6.4 und 6.5. Da dies gerade die Grenzauflösung der Daten ist, an der auch das Rauschfilter in der Bildrekonstruktion auf null geht, die Daten also gleichermaßen mit einer Punktverbreiterungsfunktion dieser Breite gefaltet sind, ist davon auszugehen, dass die wahre Größe der Strukturen noch erheblich darunter liegt. Leicht längliche Strukturen wie in Abb. 6.3 und 6.6 könnten tatsächlich von länglichem Querschnitt sein oder aber aus zwei oder mehreren nebeneinander liegenden, erheblich kleineren Strukturen bestehen. Auch die relativ kräftige magnetische Struktur in Abb. 6.11 zeigt deutliche Unterstruktur, sie scheint aus einer Aneinanderreihung mehrerer kleiner, ca. 0,5" großer Punkte zu bestehen. Die Beobachtung entsprechend kleiner Fackelpunkte im Speckle-Bild stützt dieses Ergebnis, ebenso wie Beobachtungen anderer AutorInnen z. B. von Ketten von Fackelpunkten (Filigree) im G-Band.

Ergebnis der Untersuchung ist also, dass es magnetische Strukturen auf der Sonnenoberfläche innerhalb ansonsten ruhiger Granulation gibt, die kleiner als 0,5" sind, das sind ca. $360 \mathrm{~km}$. Für die schwächsten Strukturen gerade an der Detektionsgrenze ergeben sich über die Struktur integrierte Flüsse von ca. $1 \cdot 10^{10} \mathrm{~Wb}$. Für die etwas kräftigeren Elemente, z. B. in Abb. 6.5 und 6.6, aber auch für die einzelnen Punkte der Struktur in Abb. 6.11, findet man $4-5 \cdot 10^{10} \mathrm{~Wb}$. Für den Fall, dass dabei jeweils genau eine Flussröhre beobachtet wurde, und diese einen angenommenen Durchmesser von $0,5^{\prime \prime}$ oder $360 \mathrm{~km}$ hat, entspräche das einer Flussdichte von $4 \mathrm{mT}$ für die schwachen Strukturen und $20 \mathrm{mT}$ für die etwas stärkeren. Bei einem angenommenen Durchmesser von $100 \mathrm{~km}$ wären die Flussdichten etwa $50 \mathrm{mT}$ bzw. 0,25 T. Bei diesen Abschätzungen ist zu berücksichtigen, dass die Linienschwerpunktsmethode wegen der Streulicht-Empfindlichkeit untere Grenzen für die magnetischen Flüsse gibt und auch das nur für die Sichtlinienkomponente $\left|B_{z}\right|$ der Magnetfelder. Die wahren Flussdichten $\vec{B}$ können also durchaus noch höher liegen. 


\subsection{Aktive Region (Plage)}

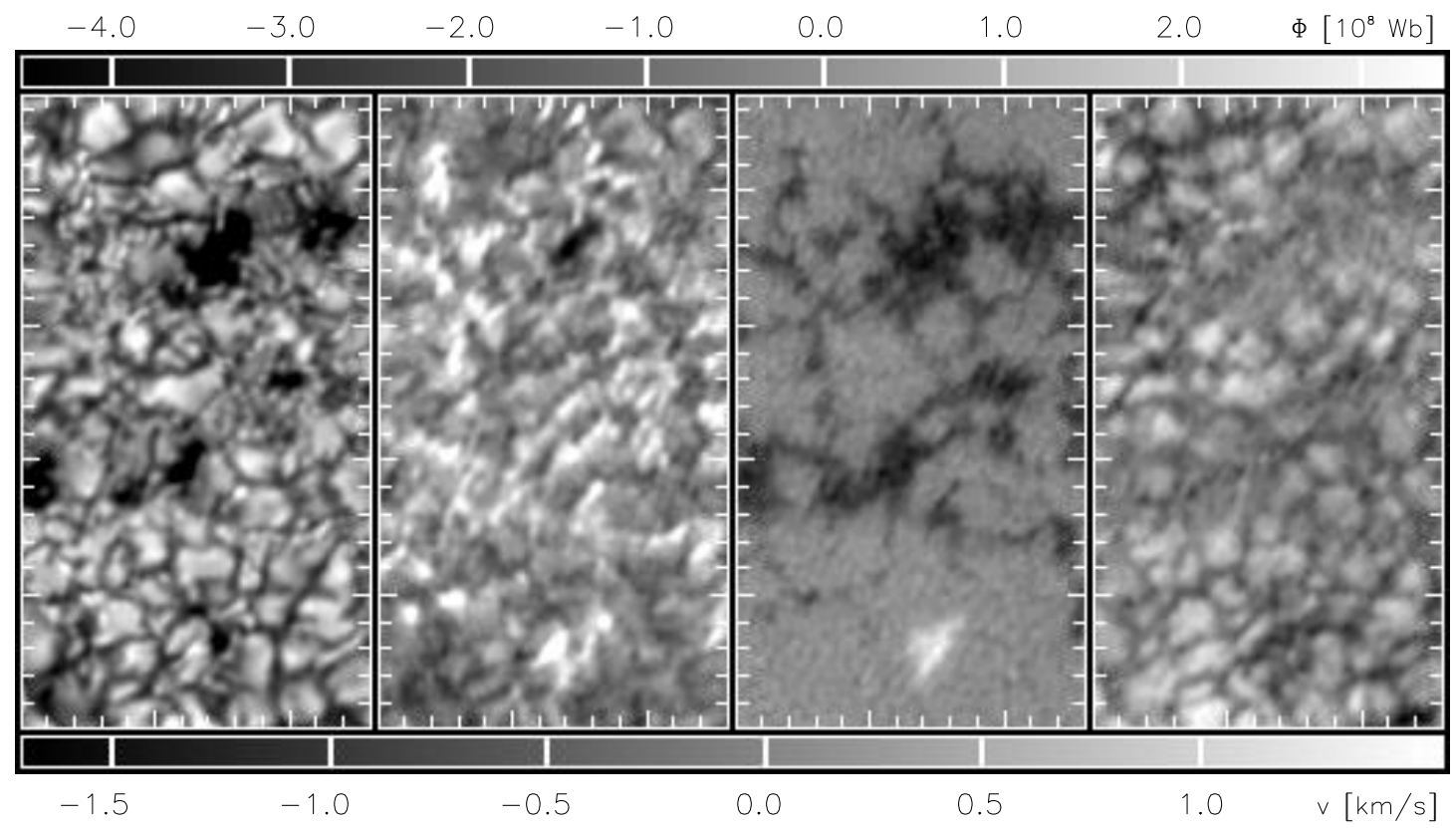

Abbildung 6.13: Von links nach rechts: Speckle-Rekonstruktion, Linienkernbild, Magnetfeldkarte und Geschwindigkeitsbild von einem aktiven Gebiet (Scan s6_6). Der Abstand der Strichmarken entspricht 1".

Das Beobachtungsgebiet der Scan-Serie s6 ist in Abb. 6.13 gezeigt. Es enthält einige kleine Poren, anomale Granulation mit vielen Fackelpunkten, aber auch Gebiete mit normaler, gut entwickelter Granulation, die allerdings auch immer wieder mit einzelnen Fackelpunkten durchsetzt ist. Das Linienkernbild zeigt an sehr vielen Stellen Aufhellungen, insbesondere dort, wo die Magnetfeldkarte Magnetfelder anzeigt. Das Bildfeld enthält ausschließlich negative Polaritäten, bis auf die etwa $2^{\prime \prime} \times 2^{\prime \prime}$ große Struktur am unteren Bildrand. Auch die Magnetfeldkarte vom Mees Solar Observatory (Abb. 4.8) zeigt für die Porengruppe nur zwei kleine, magnetisch positive Einsprengsel in ansonsten einheitlich negativer Polarität. Genau wie bei Beobachtungen von Keller \& v. d. Lühe (1992) konnten im gesamten restlichen Bildfeld auch nicht ansatzweise Strukturen positiver Polarität festgestellt werden. Lediglich in der Nähe der angesprochenen, einzelnen Struktur positiver Polarität am unteren Bildrand finden sich in einigen Scans schwache, negative Strukturen von ca. 0, 5" Durchmesser und Lebensdauern von wenigen Minuten. Ein Beispiel zeigt Abb. 6.15, in der die positive Struktur in der Speckle-Rekonstruktion als winzige, nicht voll entwickelte Pore mit Aufhellungen an ihrer linken Seite sichtbar ist. Die Magnetfeldkarte und die Stokes-V-Profile zeigen für diese Struktur kräftige Magnetfelder mit V-Amplituden von $\pm 5 \%$, nach Abb. 4.3 entspricht das wahren V-Amplituden von $\pm 10 \%$. Links neben dieser Struktur befinden sich im Abstand von ca. 1" zwei kleine Gebiete schwachen, negativen Magnetfelds. 


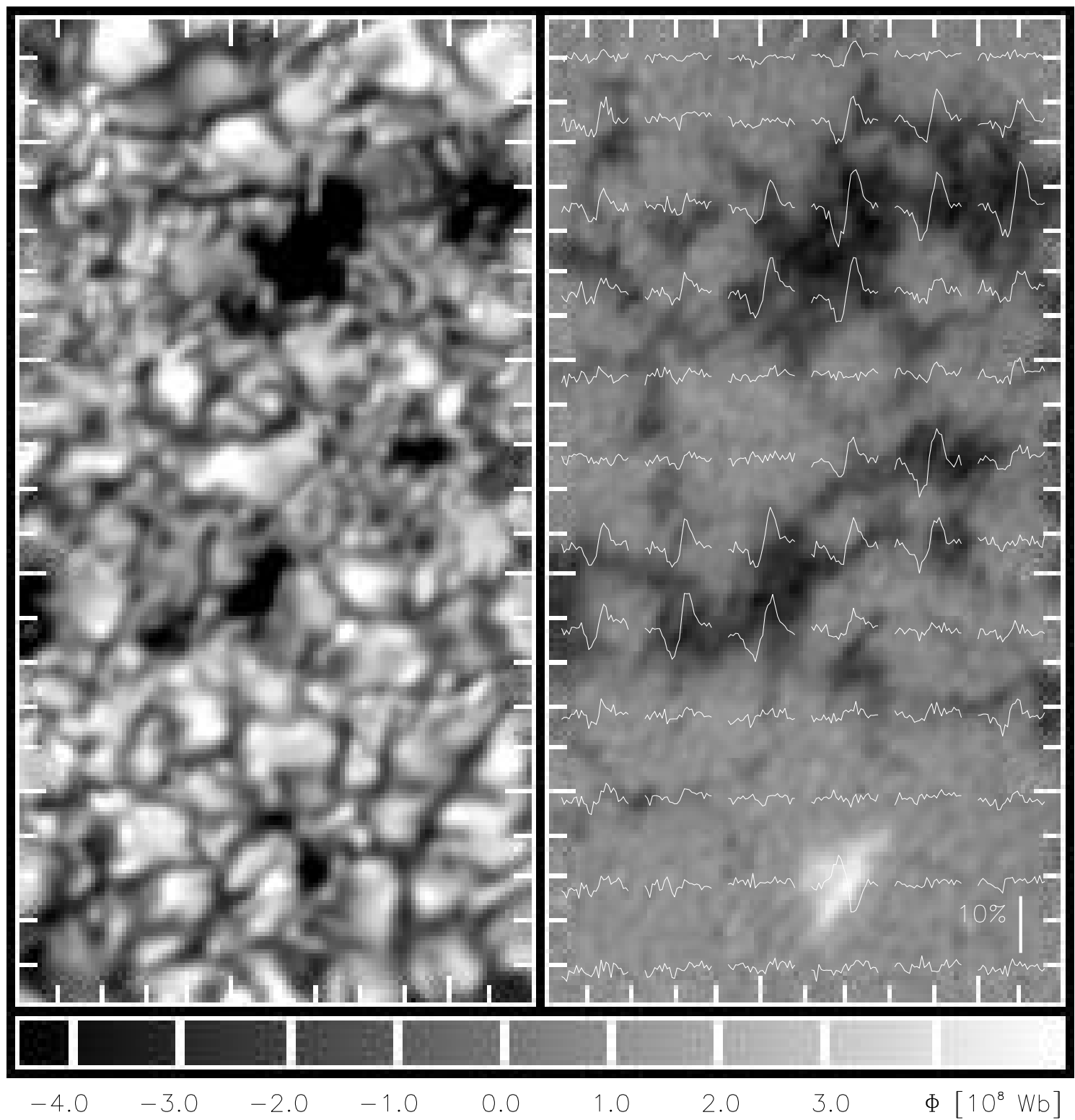

Abbildung 6.14: Links: Speckle-Rekonstruktion, rechts: Magnetfeldkarte mit Stokes-VProfilen von aktivem Gebiet (Scan s6_6). Der Abstand der Strichmarken entspricht 1".

Die stärksten Magnetfelder in Abb. 6.14 treten im Bereich der Poren in der oberen Bildhälfte mit Polarisationsgraden von $8 \%$ (bzw. nach Korrektur 16\%) auf. Die Aufspaltung der Linienschwerpunkte ergibt magnetische Flüsse von bis zu $4,2 \cdot 10^{8} \mathrm{~Wb}$ in einem Pixel. Daraus errechnen sich nach Glg. 5.18 mittlere Flussdichten der Sichtlinienkomponente von lediglich 80 mT. Dieser Wert scheint für Strukturen dieser Art um einen Faktor 2 bis 3 zu klein zu sein. Der Grund liegt in der schon in Kap. 5.2.5 angesprochenen Unterschätzung der Aufspaltung durch das Vorhandensein von nicht aufgespaltenem Streulicht in den beiden Kanälen $\frac{1}{2}\left(I_{\lambda} \pm V_{\lambda}\right)$. Der Effekt sollte für Poren am stärksten sein. Denn verglichen mit dem Streulicht von den hellen Strukturen außerhalb der Pore 


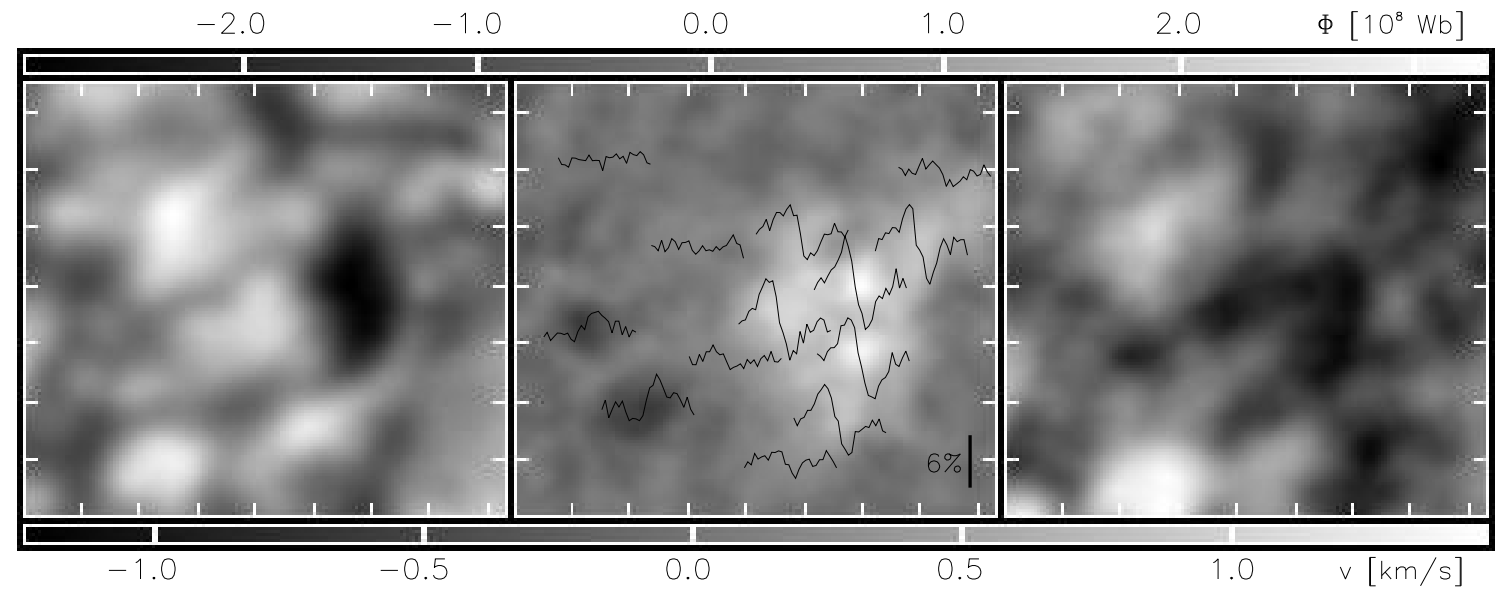

Abbildung 6.15: Ausschnitt aus Scan s6_2, links: Speckle-Rekonstruktion, Mitte: Magnetfeldkarte mit Stokes-V-Profilen, rechts: Geschwindigkeitskarte. Der Abstand der Strichmarken entspricht $0,5^{\prime \prime}$.

hat sie selbst einen geringeren Anteil eigenen Lichts, das das magnetische Signal trägt. Abb. 6.16 zeigt exemplarisch einen typischen Verlauf von Intensität, Aufspaltung der Linienschwerpunkte und Aufspaltung der Extrema der Stokes-V-Profile über eine Pore. Während der magnetische Fluss in einem Pixel im Bereich der Pore scheinbar um ca. $30 \%$ abnimmt, bleibt die Aufspaltung der Extrema der V-Profile weitgehend konstant mit einem leichten Maximum ungefähr in der Porenmitte. Aus dieser Aufspaltung errechnen sich Flussdichten von bis zu 0,22 T. Dies ist ein Wert, der gut zu bisherigen Untersuchungen passt, z. B. bei Sütterlin (1996). Gemäß der groben Abschätzung in Kap. 4.1 (Abb. 4.4) liegen diese Werte in einem Bereich, in dem die Aufspaltung der V-Profile als zuverlässiges Maß für die magnetische Flussdichte $\vec{B}$ dienen kann. Die Überschätzung der gemessenen Flussdichte aufgrund der Faltung mit dem Apparateprofil des FPIs beträgt hier wenige Prozent (vgl. Abb. 4.3). Die Diskrepanz zwischen den mittleren Flussdichten der Sichtlinienkomponente der Magnetfelder, die sich aus der Linienschwerpunktsmethode ergeben, und denjenigen Werten aus der Aufspaltung der V-Profile lässt sich also plausibel durch Streulicht erklären. Eine starke Inklination der Magnetfelder gegen die Sichtlinie, die einen ähnlichen Effekt erzeugen würde, ist für die vorliegenden Daten eher unwahrscheinlich. Beobachtungen u. a. von Martínez Pillet et al. (1997) zeigen, dass stärkere Magnetfeldstrukturen in Plage-Regionen vorwiegend senkrecht auf der Sonnenoberfläche stehen, und die vorliegenden Beobachtungen wurden nahe der Scheibenmitte gemacht. Außerdem sollten wenigstens an einigen Stellen zufällig die Feldlinien parallel zur Sichtlinie liegen und dann dort bei der Linienschwerpunktsmethode ebenfalls hohe Flussdichten ergeben. Ein weiterer Hinweis für die Richtigkeit der Annahme, dass Streulicht für die starke Unterschätzung der mittleren Flussdichte verantwortlich ist, liefern die Amplituden des Stokes-V-Signals. Diese haben, ähnlich der Aufspaltung der Linienschwerpunkte, eine Abschwächung dort, wo dunkle Poren auftreten. Da die V-Amplituden auf die Kontinuumsintensität normiert werden, muss hier 
ebenfalls mit einer Verstärkung des Effekts in dunklen Strukturen, die in eine hellere Umgebung eingebettet sind, gerechnet werden.

\subsubsection{Größe magnetischer Strukturen}

Die groben Abmessungen magnetischer Strukturen wie Poren können mit Messungen bei geringerer räumlicher Auflösung bestimmt werden, sie liegen bei den vorliegenden Daten bei $1^{\prime \prime}-3^{\prime \prime}$, es handelt sich also um eher kleine, nicht voll ausgebildete Poren. Die Frage nach einer Unterstruktur lässt sich allerdings nur bei Untersuchungen der vorliegenden Art beantworten, d. h. bei hoher räumlicher Auflösung. Schon die SpeckleRekonstruktionen im Breitbandkanal zeigen eine reichhaltige Strukturierung der Poren. Abb. 6.17 zeigt vier Poren aus dem Scan s6_18, deren Helligkeit in der Darstellung durch unscharfe Maskierung so verstärkt wurde, dass sich die innere Intensitätsstruktur der Poren noch gegen die umliegende Granulation abhebt. Strukturgrößen von $0,5^{\prime \prime}$ sind typisch. Ähnliche Strukturgrößen finden sich in den Magnetfeldkarten wieder (vgl. auch

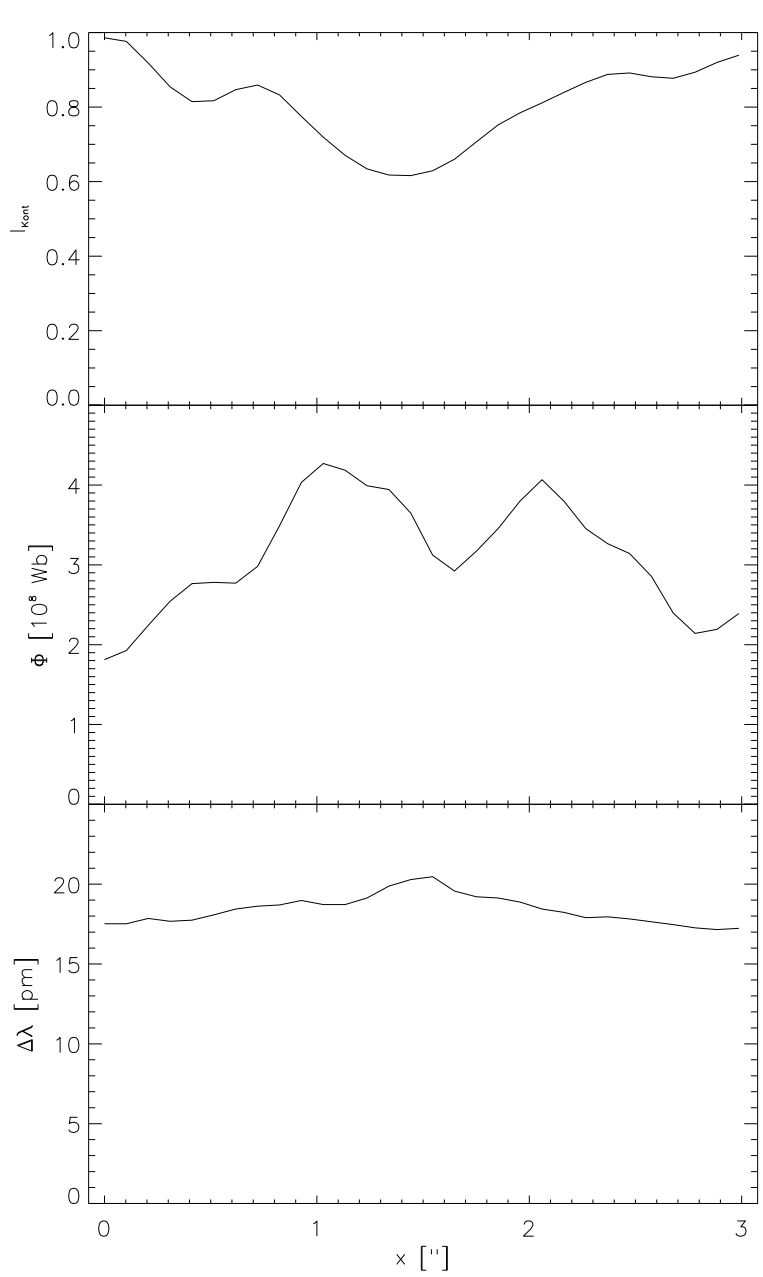

Abbildung 6.16: Verminderung des gemessenen magnetischen Flusses bei der Linienschwerpunktsmethode durch Streulicht. Oben: Verlauf der Kontinuumsintensität durch eine Pore im Scan s6_6, Mitte: magnetischer Fluss in $10^{8} \mathrm{~Wb}$ in einem Pixel, unten: Aufspaltung der Stokes-V-Extrema Abb. 6.14). Ebenso lässt sich anhand der Stokes-V-Profile zeigen, dass die magnetischen Bedingungen sich innerhalb Entfernungen von $1^{\prime \prime}$ drastisch ändern können. Abb. 6.18 zeigt einen Ausschnitt aus Scan s6_18, der die größte Pore aus dem beobachteten Gebiet enthält (oben in Abb. 6.14). Beispielsweise steigt die magnetische Feldstärke von praktisch nicht nachweisbaren Werten am unteren Bildrand (4. Profil von links) auf 0,19 T (aus der Aufspaltung des Stokes-V-Signals) nur 0,8" darüber (3. Profil von unten) am Rand der Pore. Im Inneren der Poren selbst sind die Variationen des Magnetfelds relativ gering, insbesondere treten innerhalb solcher Gebiete, die starke Magnetfelder von ca. 0,2 T aufweisen, keine kleinen feldfreien Regionen auf. Weiterhin ist festzustellen, dass die Ausdehnung der Gebiete mit starken Magnetfeldern häufig in die helle Umgebung der 


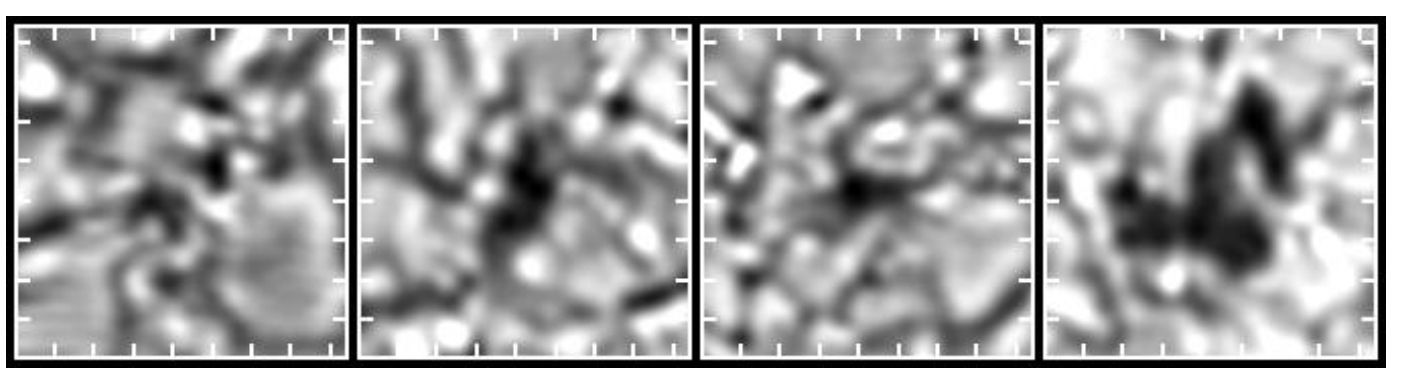

Abbildung 6.17: Speckle-Rekonstruktionen von Poren aus Scan s6_18. Der Abstand der Strichmarken ist $0,5^{\prime \prime}$.

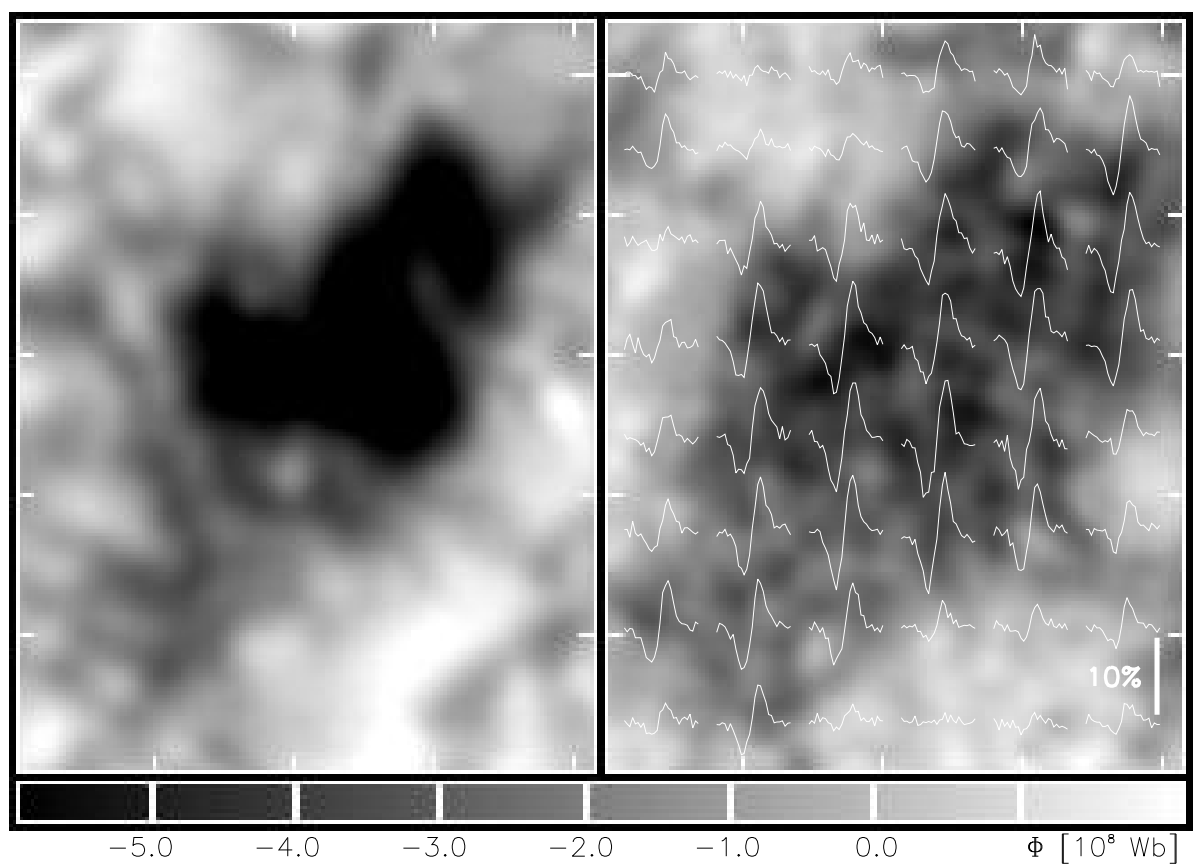

Abbildung 6.18: Ausschnitt aus Scan s6_18 mit einer Pore. Links ist die SpeckleRekonstruktion, rechts die magnetische Flusskarte mit den überlagerten Stokes-V-Profilen gezeigt. Der Abstand der Strichmarken entspricht 0, $5^{\prime \prime}$.

Poren hineinreicht, wie z. B. links unterhalb der Pore in Abb. 6.18. Mit anderen Worten, die Poren sind anhand ihres Magnetfelds nicht so scharf abzugrenzen, wie anhand ihrer Kontinuumsintensität. Eine genaue Betrachtung des Zusammenhangs zwischen magnetischem Fluss und der Intensität der Strukturen folgt weiter unten (Kap. 6.2.3).

Ebenfalls finden sich natürlich die schon aus anderen Untersuchungen (Keller \& v. d. Lühe (1992) u. a.) bekannten Fackelpunkte, kleinskalige Aufhellungen, die sich häufig entlang intergranularer Linien zu Ketten aufreihen oder längliche Strukturen geringer Breite bilden. Ein Beispiel zeigt Abb. 6.19, es handelt sich hierbei um einen Ausschnitt aus Scan s6_18 ungefähr in Bildmitte. Die Aufhellungen in der Bildmitte und rechts oben 


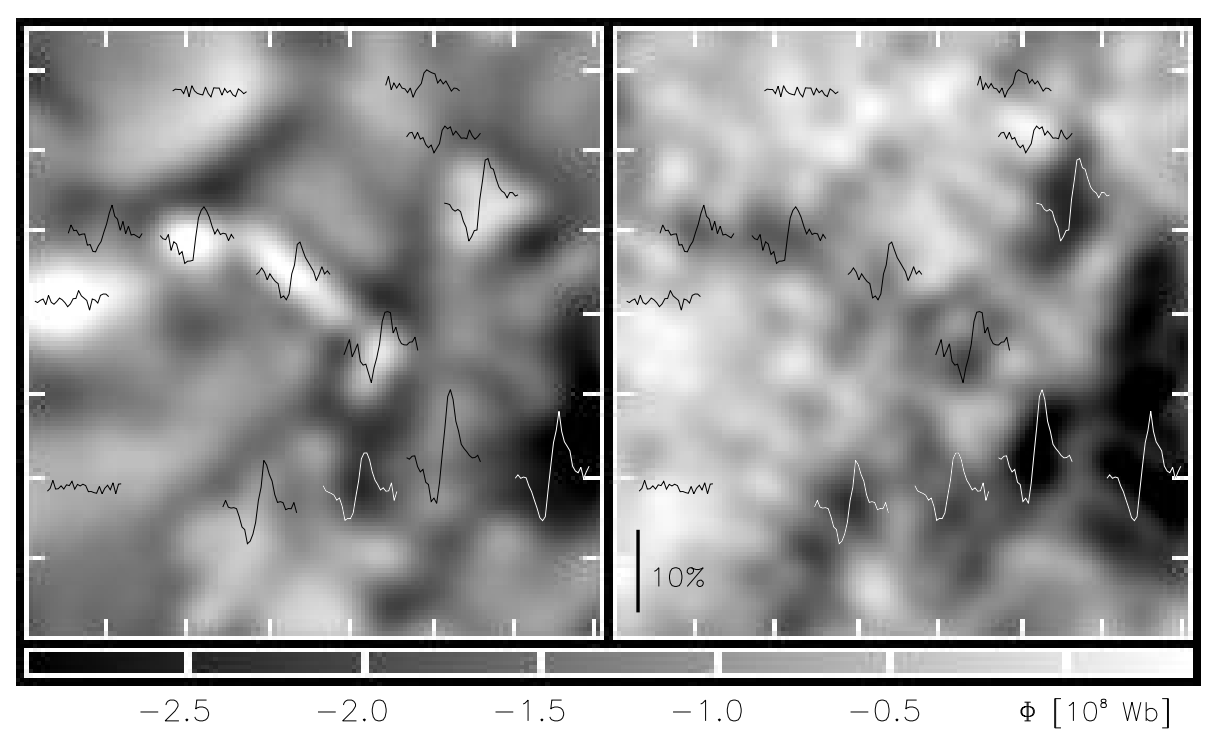

Abbildung 6.19: Fackel aus Scan s6_18. Links ist die Speckle-Rekonstruktion, rechts die magnetische Flusskarte gezeigt, jeweils mit den überlagerten Stokes-V-Profilen gezeigt. Der Abstand der Strichmarken entspricht $0,5^{\prime \prime}$.

sind mit Magnetfeldern verbunden, wie sowohl aus der Magnetfeldkarte als auch aus den überlagerten Stokes-V-Profilen ersichtlich ist. Bei genauerer Betrachtung der Struktur oben rechts in den benachbarten Scans erkennt man, dass diese aus drei einzelnen, hellen Punkten zusammengesetzt ist. Allerdings muss auch hier wieder deutlich festgestellt werden, dass es keinen strengen Zusammenhang zwischen Helligkeit der Fackelpunkte und ihrem magnetischen Fluss gibt. Die hellsten Fackelpunkte tragen häufig nicht die stärksten Magnetfelder, und an vielen Stellen finden sich Magnetfelder, die sich im Intensitätsbild nicht anhand von hellen Punkten verraten. Weiterhin muss hier erneut auf die Verwechslungsgefahr zwischen kleinen, hellen Granulen und Fackelpunkten im Intensitätsbild hingewiesen werden. Diese lassen sich häufig erst in der magnetischen Flusskarte eindeutig unterscheiden. Als Beispiel sei hier die helle Granule am linken Bildrand von Abb. 6.19 genannt.

Solche Fackelpunkte werden häufig von Scan zu Scan zwischen den Granulen hin und her geschoben. Ihre im Intensitätsbild als Aufhellung feststellbare Lebensdauer beträgt bis zu zehn Minuten, häufig kommt es zu Spaltung oder Verschmelzung einzelner Fackelpunkte. Da die messbaren Magnetfelder häufig nicht genau mit den Aufhellungen im Kontinuumsbild übereinstimmen, sondern auch die umliegenden dunklen, intergranularen Bereiche umfassen, kann über die aus dem magnetischen Signal feststellbare Lebensdauer dieser Strukturen nichts gesagt werden. Anders ausgedrückt: Die magnetische Struktur als solche kann erheblich länger bestehen als die Zeit, während der sie als Fackelpunkt oder als Pore im Kontinuumsbild sichtbar ist. Eine genauere Untersuchung der Korrelation zwischen Magnetfeldern und Aufhellungen erfolgt weiter unten in Kap. 6.2.3. 


\subsubsection{Dynamik und Lebensdauer magnetischer Strukturen}

Der schon in Kap. 6.1.5 angedeutete Zusammenhang zwischen magnetischem Fluss und Lebensdauer bzw. Dynamik scheint sich auch bei stärkeren Magnetfeldern fortzusetzen. In dem beobachteten Plage-Gebiet gibt es z. T. sehr kleine, magnetische Strukturen mit Durchmessern von 0,5" und Flüssen am Detektionslimit, wie die schon in Abb. 6.15 gezeigten. Diese bestehen häufig nur wenige Minuten und bewegen sich teilweise so schnell, dass nicht immer klar ist, welche Strukturen sich in aufeinanderfolgenden Scans entsprechen. Die Strukturen, die starke Magnetfelder enthalten, wie die Poren, aber auch die Regionen gestörter Granulation, die rings um die Poren herum zahlreich sind, sind dagegen eher beständig und zeigen wenig Dynamik. Lässt man die Speckle-Rekonstruktionen der einzelnen Scans hintereinander als Film auf dem Monitor anzeigen, so erkennt man sehr wohl eine reichhalte Dynamik kleinster Intensitätsstrukturen und der umliegenden Granulation. Die Dynamik der Intensitätsstrukturen kann dabei sowohl von der granularen Strömung herrühren, wie auch von Schwankungen der Helligkeit bestimmter Strukturen am Rande oder innerhalb von Poren. Ein entsprechender Film der magnetischen Flusskarten wirkt dagegen ausgesprochen ruhig und beständig. Während der gesamten, 25 Minuten umfassenden Zeitserie sind keine gravierenden Veränderungen der Anordnung und mittleren Stärke der Magnetfelder festzustellen. Abb. 6.20 zeigt eine Zeitserie der Pore aus Abb. 6.15. Deutlich ist der ständige Wandel des Aussehens der Pore im Integralbild zu erkennen. Die Pore wird im Verlauf der 20 Scans deutlich schwächer und kleiner, ungefähr ab dem 13. Scan teilt sie sich. Teilweise sind in direkter Nähe der Pore kleinskalige Aufhellungen zu erkennen (8.-12. Scan). Dagegen fällt in den Karten des magnetischen Flusses die Ruhe und Beständigkeit der Struktur auf, der Gesamtfluss in diesem Gebiet bleibt ebenfalls die ganze Zeit über ungefähr konstant auf einem Wert von $10^{12} \mathrm{~Wb}$.

Im Gegensatz zu den sehr starken Magnetfeldern geringer Dynamik gibt es aber sehr wohl Strukturen mittlerer Flusswerte (etwa $1-2 \cdot 10^{8} \mathrm{~Wb}$ in einem Pixel, etwa $2 \cdot 10^{11} \mathrm{~Wb}$ insgesamt), die deutliche Bewegung zeigen. Diese sind allerdings selten. Ein Beispiel ist in Abb. 6.21 gezeigt, es handelt sich dabei um einen Ausschnitt aus der Aufnahmeserie s6, und zwar um die schwache Struktur negativer Polarität am linken Bildfeldrand (vgl. hierzu Abb. 6.14) etwa 5" oberhalb des unteren Bildfeldrands. Die magnetische Struktur, die sich anfangs am rechten Bildrand befindet, wandert im Verlauf der folgenden 14 Scans um ca. 1" nach links oben, um ab dem 12. Scan ihre Form völlig zu verändern.

\subsubsection{Zusammenhang zwischen magnetischem Fluss und Intensität}

Ähnlich den Untersuchungen in Kap. 6.1.3 in ruhiger Granulation soll nun für die PlageRegion ein quantitativer Zusammenhang zwischen magnetischem Fluss auf der einen Seite und Intensität in Kontinuum und Linienkern bzw. Geschwindigkeiten auf der anderen Seite untersucht werden. Abb. 6.22 und Abb. 6.23 zeigen, dass sich das schon 


\subsection{Aktive Region (Plage)}

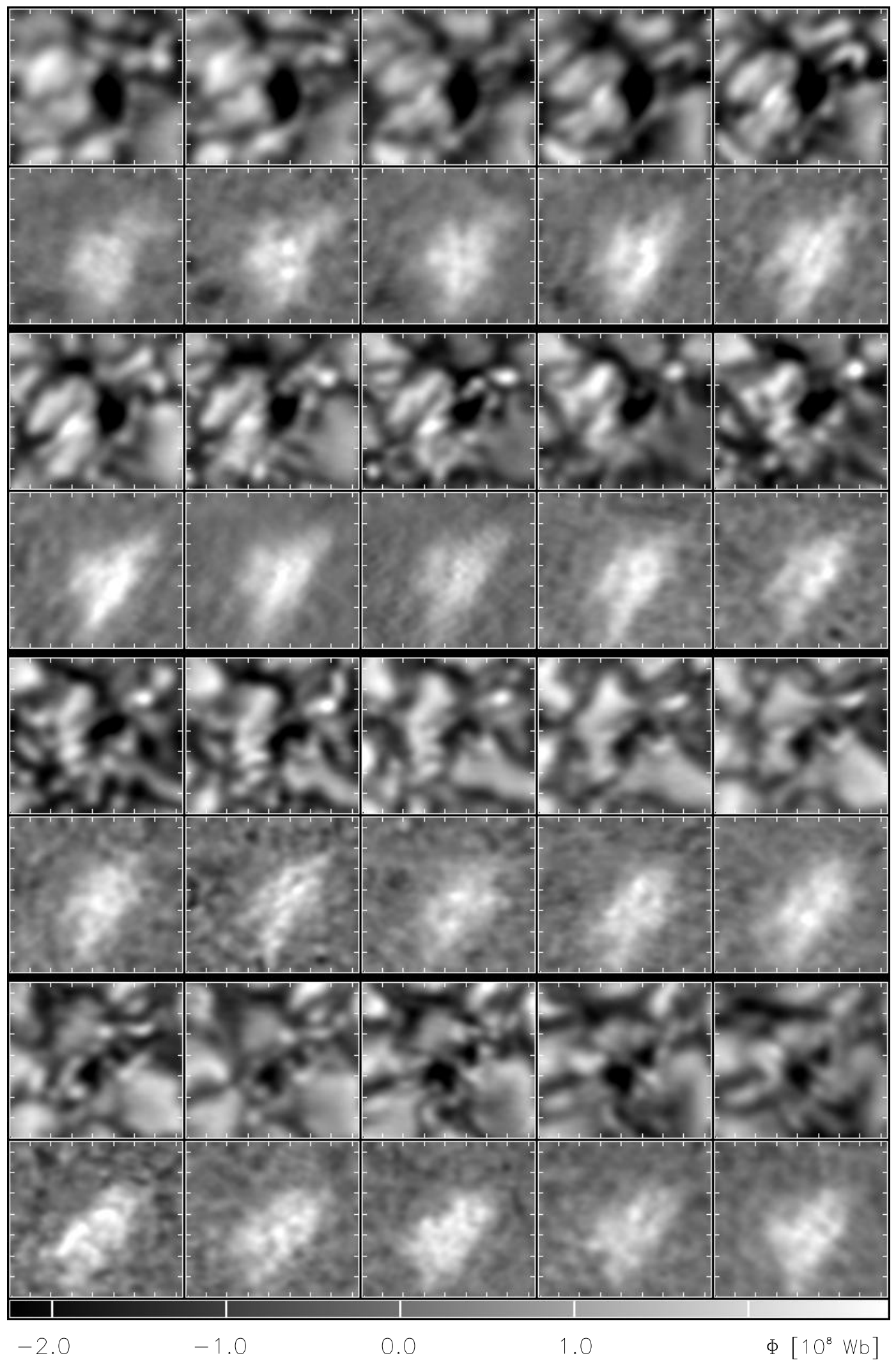

Abbildung 6.20: Zeitserie der Pore aus Abb. 6.15. Die Scans sind zeilenweise von links oben nach rechts unten angeordnet. Unter den Speckle-Rekonstruktionen befindet sich jeweils die Karte des magnetischen Flusses. Die Helligkeitsskalierung aller Bilder ist identisch. Der Abstand der Strichmarken entspricht $0,5^{\prime \prime}$. 


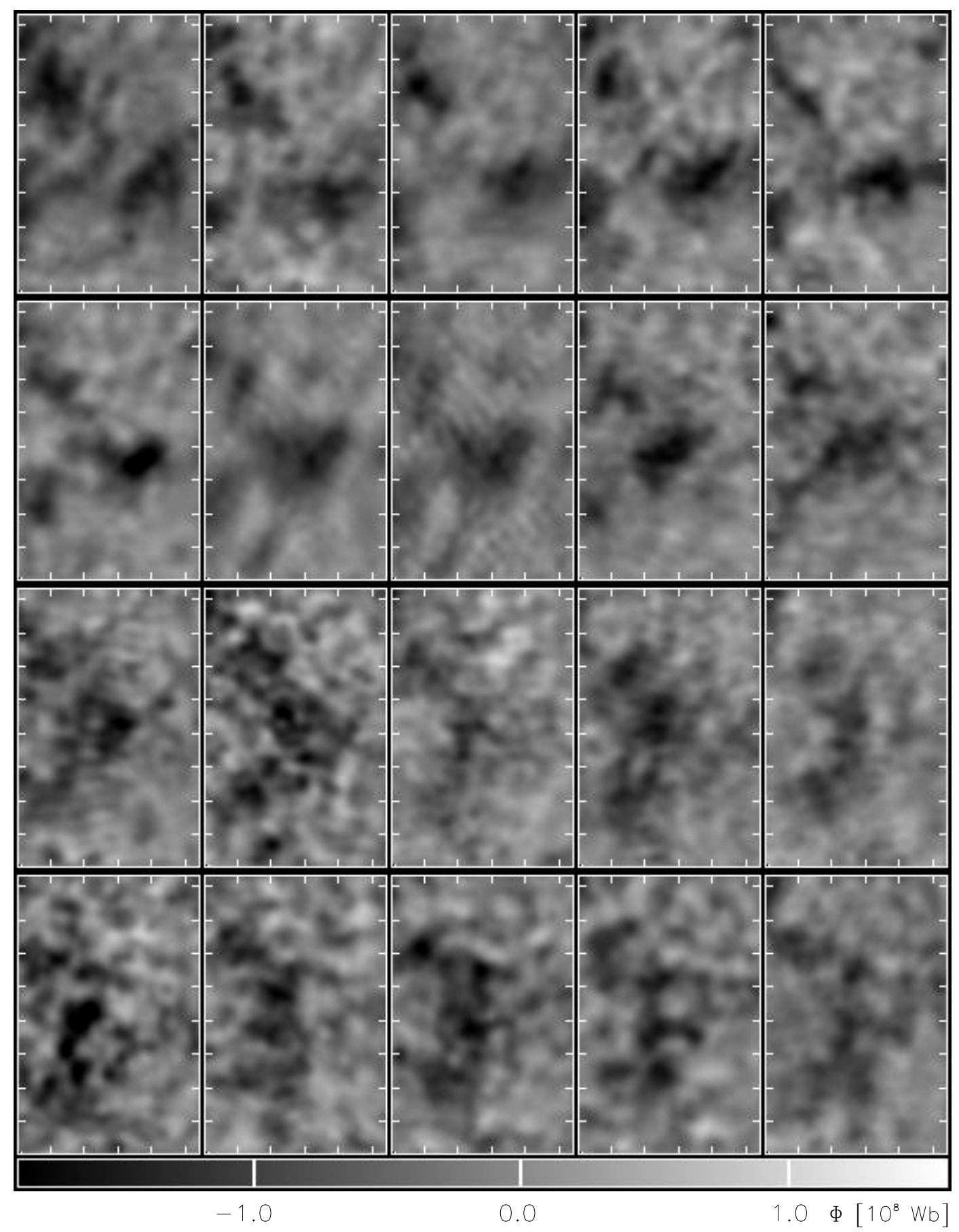

Abbildung 6.21: Zeitserie eines Ausschnitts der magnetischen Flusskarten aus Serie s6, die Scans sind zeilenweise von links oben nach rechts unten angeordnet. Die Helligkeitsskalierung aller Bilder ist identisch. Der Abstand der Strichmarken entspricht 0,5". 


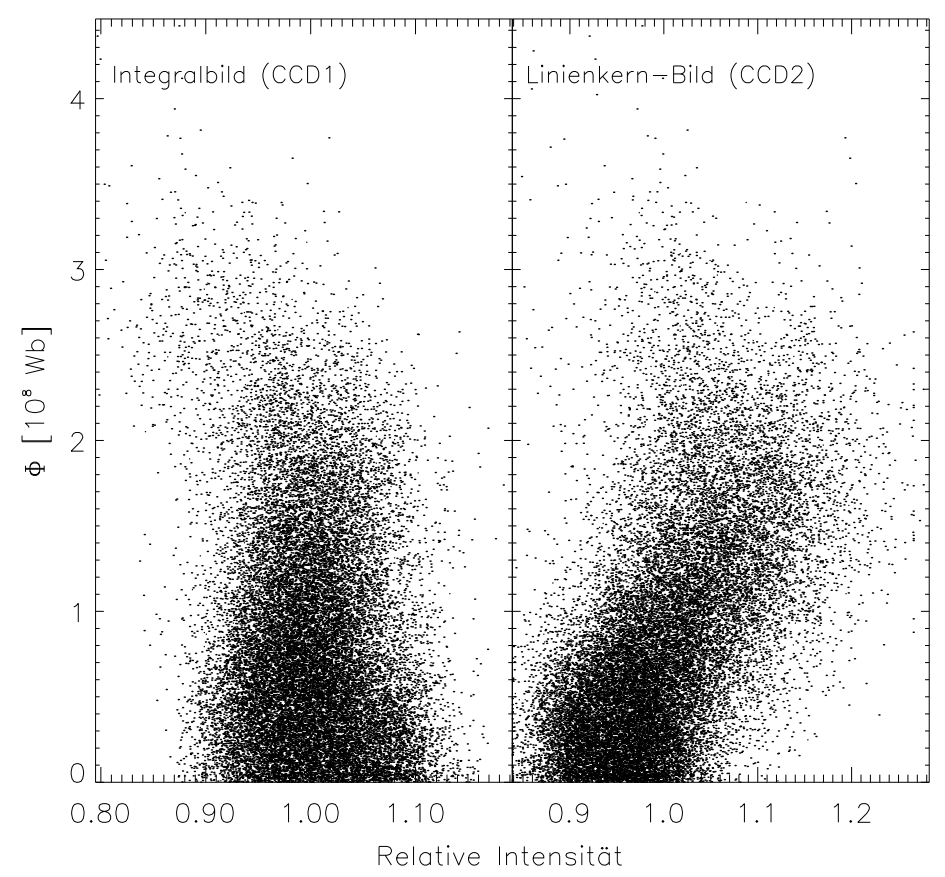

Abbildung 6.22: Zusammenhang zwischen magnetischem Fluss und Intensität für einzelne Pixel aus anomaler Granulation der Plage-Scans s6. Links sind die Intensitäten im Integralbild benutzt, rechts die im Linienkern.

in der ruhigen Granulation festgestellte Verhalten der Intensität magnetischer Strukturen in Plage-Regionen kontinuierlich zu stärkeren Magnetfeldern hin fortsetzt. Oberhalb sehr schwacher Felder von $0,5 \cdot 10^{8} \mathrm{~Wb}$ in einem Pixel ist eine geringe Abnahme der Häufigkeit heller Strukturen feststellbar, was durch das Fehlen von Magnetfeldern in den hellsten Granulen zu erklären ist. Im Bereich zwischen 0,5 und 2,0 $\cdot 10^{8} \mathrm{~Wb}$ in einem Pixel sind sowohl dunkle als auch mäßig helle magnetische Strukturen vorhanden, mit moderaten Abweichungen von der mittleren Kontinuumsintensität (durchgezogene Linien in Abb. 6.23). Die Linienkernintensität nimmt im gleichen Bereich jedoch ungefähr linear mit dem magnetischen Fluss zu (gestrichelte Linien in Abb. 6.23). Oberhalb von 2, $0 \cdot 10^{8} \mathrm{~Wb}$ in einem Pixel ist jedoch eine deutliche Veränderung des Zusammenhangs erkennbar: Sowohl in den Kontinuums- wie auch in den Linienkernbildern ist nun eine Abnahme der Intensität mit weiter steigendem magnetischen Fluss zu beobachten. Dabei sind in diese Untersuchung ausschließlich Gebiete mit anomaler Granulation und Fackelpunkten eingegangen. Nimmt man auch die Poren selbst mit hinzu, so machen sich diese ab etwa 2, $5 \cdot 10^{8} \mathrm{~Wb}$ in einem Pixel mit einem deutlichen Knick in den beiden Kurven nach links bemerkbar. Dies ist in Abb. 6.24 dargestellt. Dazu ist noch zu bedenken, dass aufgrund der schon geschilderten Unterschätzung der magnetischen Flüsse wegen Streulichts besonders in den Poren diese mit etwas höheren Flüssen anzusetzen wären. 

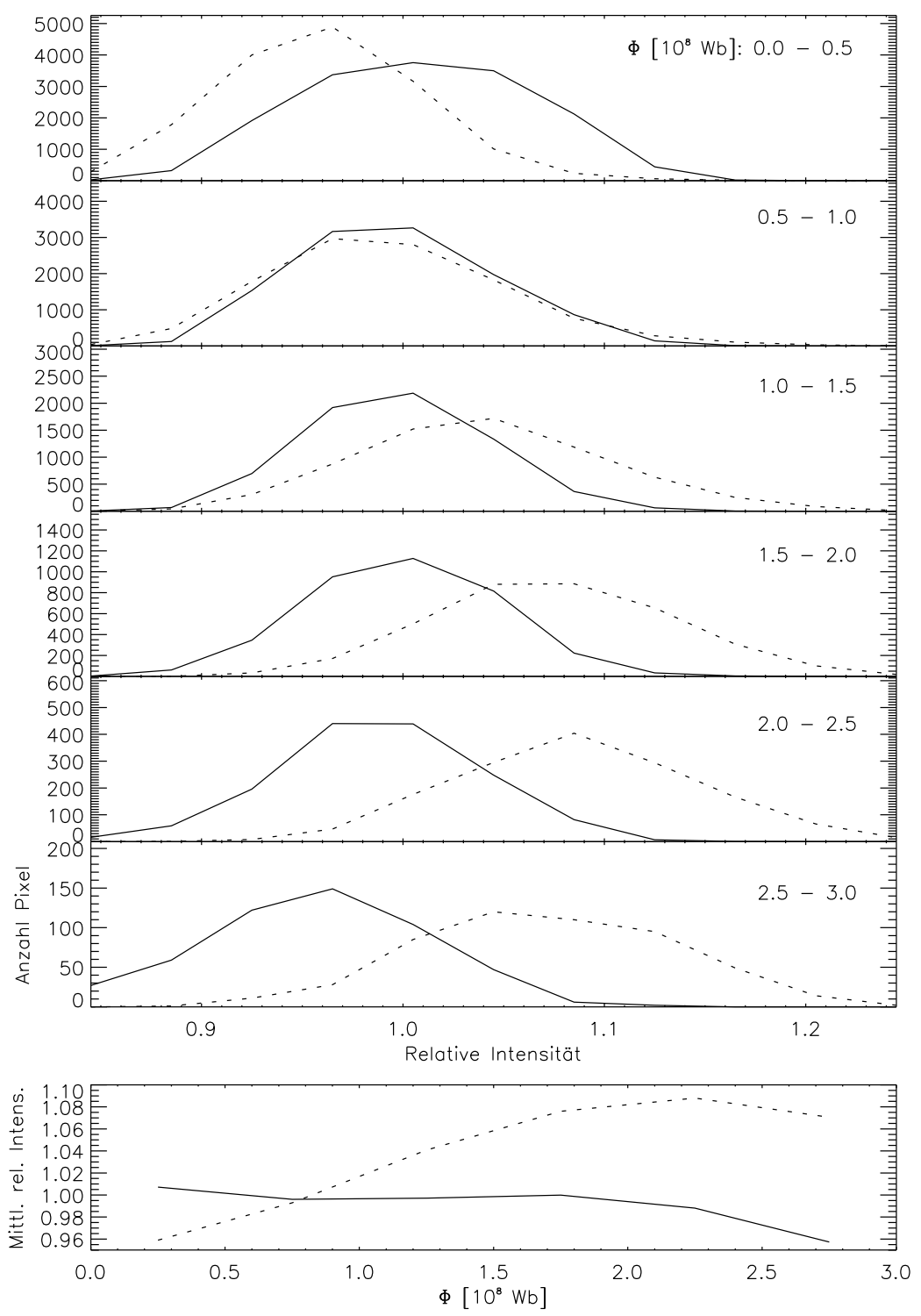

Abbildung 6.23: Histogramme der Intensitätsverteilung für verschiedene magnetische Flüsse in der Plage-Region. Durchgezogen sind jeweils die Häufigkeiten von Pixeln bestimmter Kontinuumsintensität aufgetragen, gestrichelt für die Linienkernintensitäten, von oben nach unten für steigenden magnetischen Fluss. In der untersten Auftragung sind die gemittelten Intensitäten für die obigen Flussbereiche gezeigt.

Zusammenfassend für ruhige Granulation und Plage-Gebiete einschließlich Poren kann gesagt werden, dass schwache Magnetfelder unterhalb $2,0 \cdot 10^{8} \mathrm{~Wb}$ in einem Pixel im Kontinuum sowohl in hellen, wie auch in dunklen Strukturen vorkommen, allerdings größte Helligkeiten wie in sehr hellen, unmagnetischen Granulen unterrepräsentiert sind. Schon Keller \& v. d. Lühe (1992) haben gefunden, dass magnetische Strukturen nicht unbedingt hell sein müssen. In Linienkernbildern verraten sich solche schwächeren Ma- 


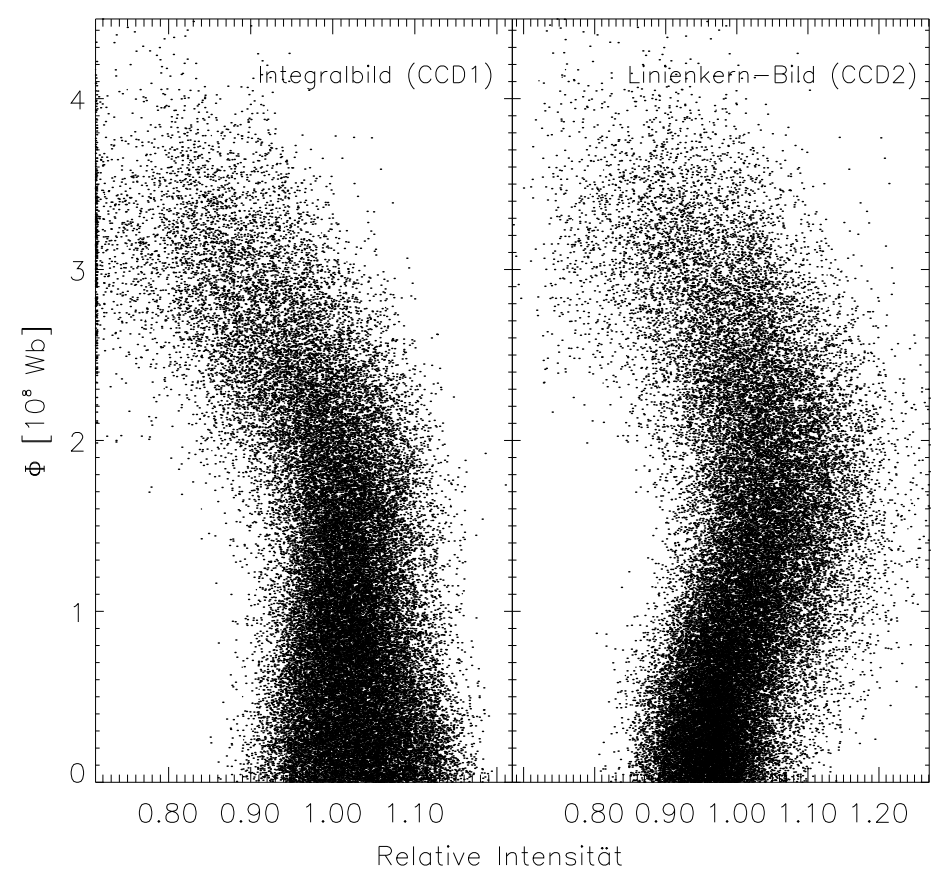

Abbildung 6.24: Zusammenhang zwischen magnetischem Fluss und Intensität für einzelne Pixel der Plage-Scans s6. Hier sind auch Datenpunkte aus Poren verwendet worden. Links sind die Intensitäten in der Speckle-Rekonstruktion benutzt, rechts die im Linienkernbild.

gnetfelder durch Aufhellungen, die um so stärker sind, je stärker das Magnetfeld ist. Magnetische Strukturen mittlerer Stärke $\left(1,5-2,5 \cdot 10^{8} \mathrm{~Wb}\right.$ in einem Pixel) zeigen sich häufig als mäßig helle Fackelpunkte in Kontinuumsbildern. Oberhalb eines magnetischen Flusses von 2,5 $\cdot 10^{8} \mathrm{~Wb}$ in einem Pixel werden die Strukturen aber zunehmend dunkler und gehen fließend in Poren über. In den in Kap. 4 gezeigten Aufnahmen der höheren Atmosphärenschichten der Sonne ( $\mathrm{H} \alpha$, Ca K, EIT-Bilder) ist, bei deutlich schlechterer räumlicher Auflösung, das Aktivitätsgebiet ebenfalls als Aufhellung erkennbar.

Bedacht werden sollte noch die Möglichkeit, dass es auf tiefem, photosphärischem Niveau, aus dem das Licht der Integralbilder im Mittel entstammt, durchaus eine Korrelation zwischen Magnetfeld und Intensität auch für schwächere Magnetfelder existieren könnte. Diese würde in der vorliegenden Untersuchung u. U. aber nicht registriert werden können, da das magnetische Signal aus der Spektrallinie bei 630,25 nm und somit aus höheren atmosphärischen Schichten stammt. In diesen Höhen könnte durch die Aufweitung der magnetischen Flussröhren („Canopy-Effekt“) der Zusammenhang aus tieferen Schichten verloren gehen. Genauere Inspektion einzelner Strukturen zeigt aber nicht das dann zu erwartende Bild, dass jeweils z. B. um helle Strukturen herum immer eine ausgedehntere magnetische Struktur zu beobachten wäre. Im Gegenteil finden sich räumlich eng begrenzte, magnetische Strukturen von $0,5^{\prime \prime}$ oder weniger Durchmesser, die 
deutlich in dunklen, intergranularen Gebieten liegen, ohne darunter liegende oder benachbarte Fackelpunkte, aus denen das ursprünglich auf diese konzentrierte Magnetfeld sich hätte aufweiten oder seitlich verschieben können. Es ist deshalb aufgrund dieser Messergebnisse anzunehmen, dass schwache bis mittelstarke Magnetfelder tatsächlich sowohl in hellen Fackelpunkten als auch in dunkleren Strukturen existieren. Sicherheit könnte in diesem Punkt eine Untersuchung solcher Strukturen mit einer tiefer in der Photosphäre entstehenden Spektrallinie brin-

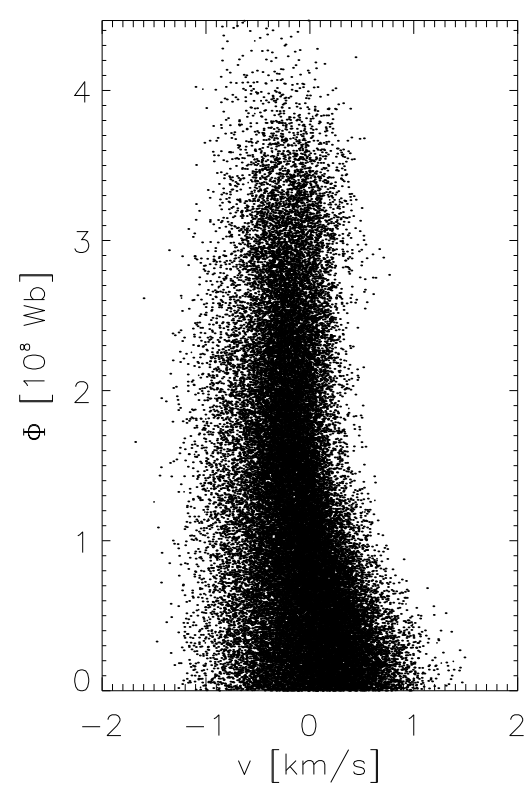

Abbildung 6.25: Zusammenhang zwischen magnetischem Fluss und Geschwindigkeiten für einzelne Pixel der Plage-Scans s6. gen. Selbstverständlich gilt diese Aussage auch nur für räumliche Auflösungen bis zu etwa 0, $5^{\prime \prime}$, für kleinere Strukturen könnten durchaus andere Abhängigkeiten gefunden werden.

\subsubsection{Zusammenhang zwischen magnetischem Fluss und Strömungen}

Wie schon in Kap. 6.1.4 soll nun auch für die Plage-Region einschließlich der Poren die Abhängigkeit der Strömungsgeschwindigkeiten (bzw. deren Sichtlinienkomponente) von der Stärke des magnetischen Flusses untersucht werden. Und wie schon bei den Intensitäten entspricht das Verhalten dem in ruhiger Granulation, außer dass nun noch stärkere Magnetfelder auftreten. Das Ergebnis ist, dass für Strukturen oberhalb von $2,0 \cdot 10^{8} \mathrm{~Wb}$ in einem Pixel die mittlere Geschwindigkeit wie auch die Geschwindigkeitsverteilung weitgehend konstant bleibt, bei einer mittleren Abströmung von $200 \mathrm{~m} / \mathrm{s}$ und einer Streuung zwischen $-1 \mathrm{~km} / \mathrm{s}$ und $+0,5 \mathrm{~km} / \mathrm{s}$ (Abb. 6.25 und 6.26). 
6.3 Kleiner Sonnenfleck
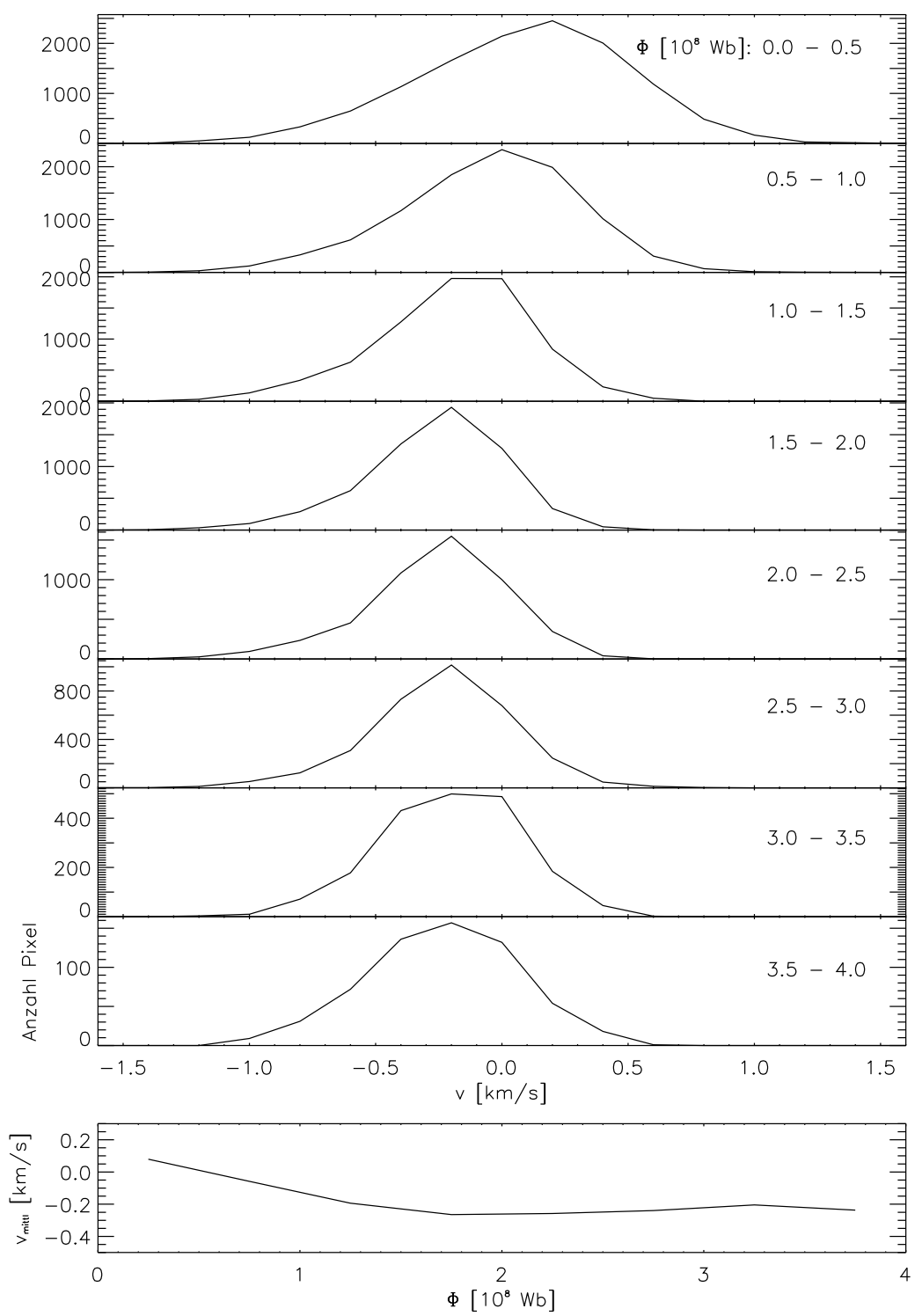

Abbildung 6.26: Histogramme der Geschwindigkeitsverteilung für verschiedene magnetische Flüsse. In der untersten Auftragung sind die gemittelten Geschwindigkeiten für die obigen Fluss-Bereiche gezeigt.

\subsection{Kleiner Sonnenfleck}

Als letztes Beobachtungsobjekt soll ein kleiner Sonnenfleck gezeigt werden, der am 22.10. 1999 zusammen mit der Plage-Region aus Kap. 6.2 eine kleine, bipolare Region bildete (NOAA 8738). Genaueres zur Lage des Flecks und der aktiven Gruppe findet sich in Kap. 4.2.

Da dieser kleine Fleck (vgl. auch Abb. 6.3) Ansätze einer Penumbra zeigt, ist davon auszugehen, dass die Magnetfelder häufig nicht geringe Inklinationen $\gamma$ haben, also recht 
starke Neigungen der Feldlinien gegen die Senkrechte auf der Sonnenoberfläche und damit in diesem Fall gegen die Sichtlinie. Für ausgeprägte Sonnenflecken erwartet man Inklinationen von $\gamma \approx 80^{\circ}$ (Degenhardt \& Wiehr (1991)). In dem vorliegenden Fall würden also vermutlich wegen der Kleinheit und Unregelmäßigkeit des Flecks alle Werte zwischen $0^{\circ}$ und $80^{\circ}$ auftreten.

Bei solchen Neigungswinkeln der magnetischen Feldlinien gegen die Sichtlinie entsteht für das vorliegende Beobachtungskonzept das Problem, dass der transversale ZeemanEffekt auftritt und somit linear polarisierte Licht-Komponenten vorhanden sein sollten. Diese können bei den magnetischen Feldstärken und Inklinationen in Sonnenflecken bzw. Penumbren erhebliche Anteile des polarisierten Lichts ausmachen. Im Magnetogramm der transversalen Feldkomponente vom Mees Solar Observatory der aktiven Region an diesem Tag (Abb. 4.8 unten links) sind diese linear polarisierten Anteile im Bereich des Flecks deutlich zu erkennen.

Wie schon in Kap. 3.3 beschrieben, ist ein Coelostaten-System wie das im VTT verwendete nicht frei von instrumenteller Polarisation und gegenseitigem Übersprechen der Komponenten des Stokes-Vektors. Insbesondere sind die genauen Werte der MuellerMatrix $M_{42}$ und $M_{43}$, die das Übersprechen von linear polarisierten Komponenten in zirkular polarisierte beschreiben, von verschiedenen Faktoren abhängig und deshalb im vorliegenden Fall unbekannt.

Aus diesem Grunde müssen die gemessenen Magnetfelder im Sonnenfleck und seiner nächsten Umgebung mit Vorsicht betrachtet werden, vor allem können durch das Übersprechen von symmetrischen Stokes-Q- und -U-Profilen in die antisymmetrischen StokesV-Profile Asymmetrien vorgetäuscht werden, die in Wirklichkeit nicht vorhanden sind.

Es soll deshalb hier nur eine grobe Beschreibung der magnetischen Eigenschaften des Sonnenflecks und seiner Umgebung gegeben werden. Genauere Untersuchungen solcher Objekte müssen Gegenstand zukünftiger Untersuchungen sein, bei denen zumindest die instrumentelle Polarisation und der Crosstalk der Beobachtungsanlage bestimmt werden, besser noch der gesamte Stokes-Vektor gemessen wird.

Abb. 6.27 zeigt den Sonnenfleck in seinen verschiedenen Eigenschaften. In der SpeckleRekonstruktion ist ein dunkler Kernbereich zu erkennen, der aber deutliche Anzeichen kleinskaliger Aufhellungen zeigt. Dies sind die bekannten „Umbral Dots“, die an anderer Stelle ausführlicher beschrieben sind (Denker (1996) u. a.). Ihre Größe geht hinunter bis zur räumlichen Auflösungsgrenze dieser Beobachtungen. Aus den vorliegenden Daten lässt sich allerdings kein Zusammenhang erkennen, ob die Umbral Dots mit stärkeren oder schwächeren Magnetfeldern verbunden sind.

Das Geschwindigkeitsbild in Abb. 6.27 ganz rechts zeigt deutlich die Unterdrückung der granularen Konvektion im Bereich der kräftigen Magnetfelder.

In den Randbereichen des Flecks, in denen sich dunkle, porenartige Strukturen mit heller, anomaler Granulation abwechseln, finden sich auch einige penumbrale Filamente, deren Intensität zwischen denen des Flecks und der Granulation liegt. Diese sind 


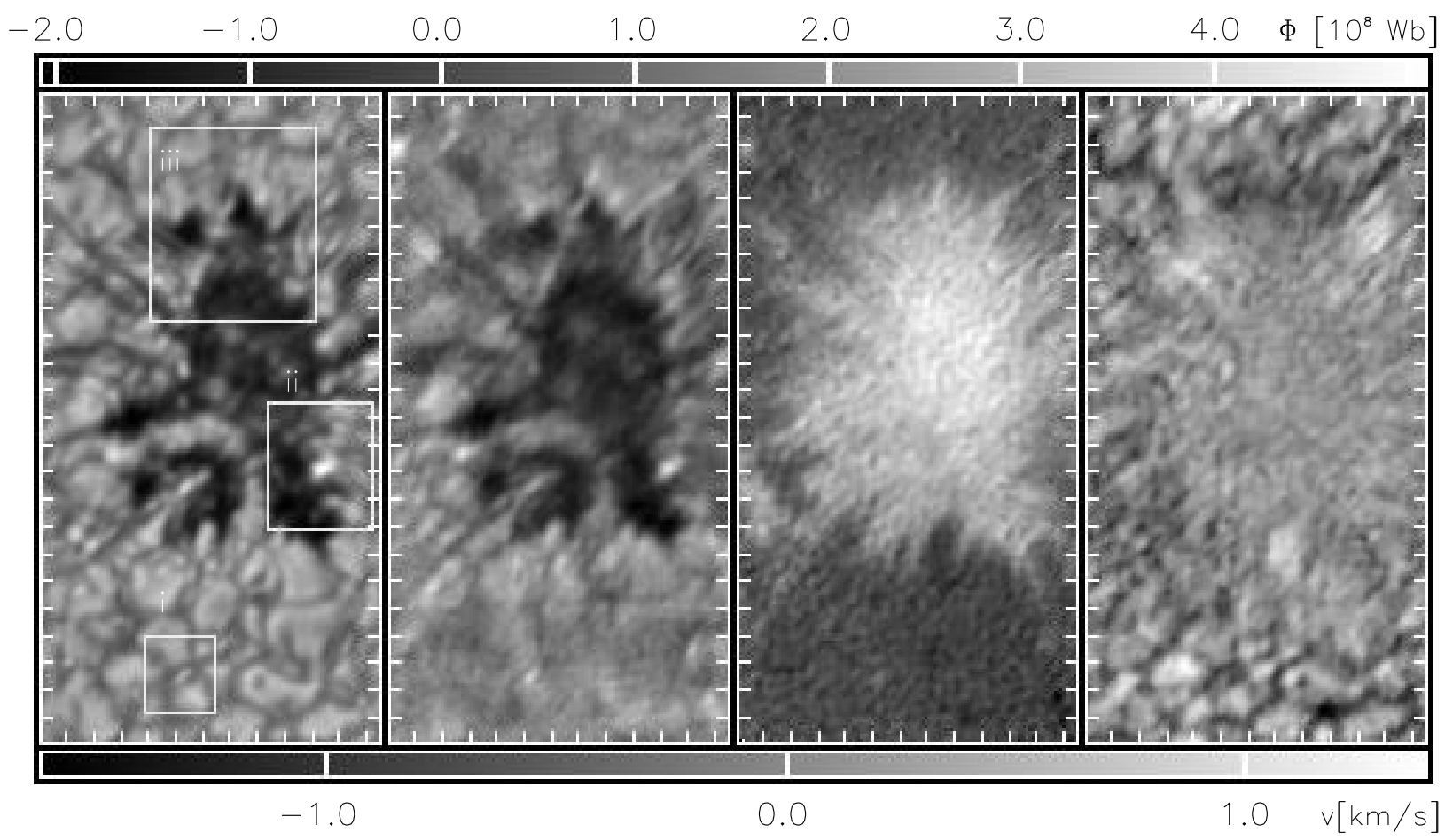

Abbildung 6.27: Kleiner Sonnenfleck aus Scan s4_9. Von links nach rechts sind SpeckleRekonstruktion, Linienkernbild, Karte des magnetischen Flusses und Geschwindigkeitsbild gezeigt. Der Abstand der Strichmarken entspricht 1".

auch im Linienkern deutlich zu erkennen. Das Linienkernbild zeigt weiterhin den Fleck und den porenartigen, ausgefransten Rand, dazu im Randbereich einige Aufhellungen, die auch in der Speckle-Rekonstruktion zu erkennen sind (vgl. Abb. 6.29). Im Bereich der Granulation ist eine sehr gleichmäßige Intensität zu verzeichnen, bis auf eine kleine Aufhellung am unteren Bildrand, die sich in der magnetischen Flusskarte als schwache magnetische, intergranulare Struktur zeigt. Die Speckle-Rekonstruktion zeigt an dieser Stelle einen länglichen Fackelpunkt. Diese Struktur, die in Abb. 6.27 mit i markiert ist, ist in Abb. 6.28 vergrößert dargestellt. Sie zeigt schwache, positive Stokes-V-Profile und unterscheidet sich in ihren Eigenschaften nicht von ähnlichen Strukturen in den Scans s5 in einem ruhigen, unmagnetischen Gebiet. Dies passt zu der Beobachtung, dass die magnetische Flusskarte aus Abb. 6.27 im Bereich der Granulation unterhalb des Flecks keine nennenswerten Magnetfelder zeigt, bis auf die in Abb. 6.28 gezeigte und eine weitere, ähnliche Struktur rechts unterhalb des Flecks. Diese befindet sich ebenfalls in einem Intergranulum, hat allerdings eine dem Fleck entgegengesetzte Polarität. Insbesondere sind in diesem Granulationsausschnitt keine Anzeichen von (umgekehrtem) Fluss zu entdecken, der seinen Ursprung im Fleck hat und in der umgebenden Granulation abtaucht, wie es z. B. in Modellrechnungen von Osherovich (1982 angenommen wird, der allerdings von einem symmetrischen, einzeln stehenden Fleck ausgeht. 


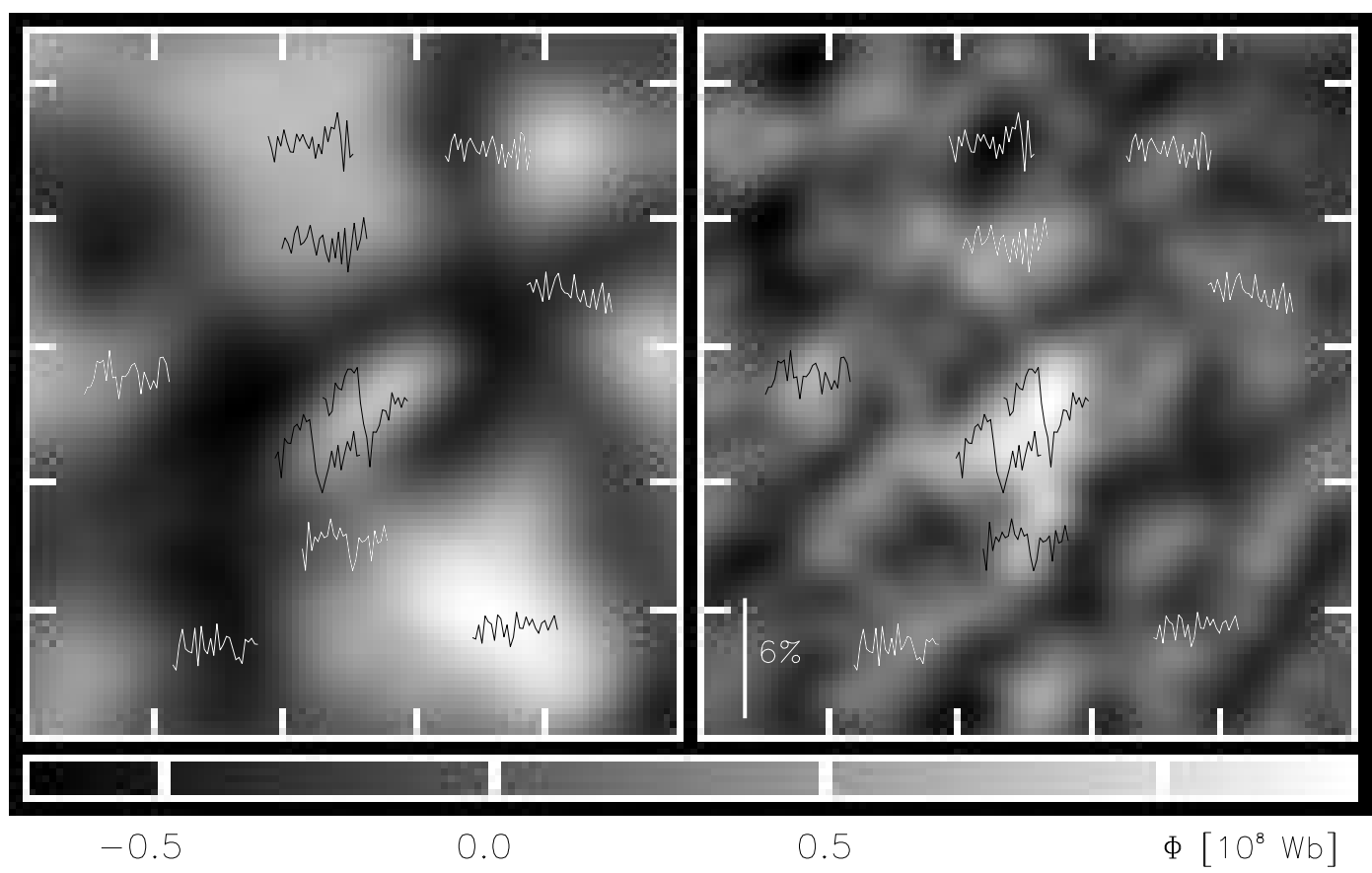

Abbildung 6.28: Ausschnitt i aus Abb. 6.27 mit kleiner magnetischer Struktur im Bereich der Granulation in der Nähe des Sonnenflecks. Links die Speckle-Rekonstruktion, rechts die Magnetfeldkarte. Der Abstand der Strichmarken entspricht 0,5".

Im Bereich des Flecks und der Penumbra zeigt die Magnetfeldkarte kräftige Magnetfelder. Bei einem angenommenen Füllfaktor $\alpha=1$ ergeben sich für die Sichtlinienkomponente der Felder aus der Linienschwerpunktsmethode Flussdichten von maximal 0,1 T, was wiederum für eine solche Struktur zu wenig erscheint. Wie schon in Kap. 6.2 ist Streulicht die Ursache für diese Unterschätzung. Aus der Aufspaltung der Stokes-VProfile ergeben sich hier im Kernbereich des Flecks Feldstärken von bis zu 0,23 T, was gängigen Vorstellungen entspricht.

Abb. 6.29 zeigt das in Abb. 6.27 mit ii markierte Gebiet mit der Aufhellung in Kontinuum und Linienkern, Abb. 6.30 zeigt die obere Hälfte des Flecks mit penumbralen Filamenten und angrenzender Granulation (mit iii markiert). Beide Bilder zeigen im Bereich der starken Magnetfelder im Fleck und am Rand des Flecks keine starken Variationen des Magnetfelds, insbesondere keine deutliche Korrelation zwischen kleinskaligen Helligkeits- und Magnetfeldfluktuationen, wie gerade auch die aufgehellte Struktur in Abb. 6.29 deutlich macht. Lediglich ein grober Trend ist zu beobachten, dass, je weiter man zum Inneren des Flecks kommt, die Intensität abnimmt und die Magnetfelder stärker werden. Allerdings muss hierbei bedacht werden, dass in diesem Zusammenhang Amplitude des Stokes-V-Signals bzw. Aufspaltung der Linienschwerpunkte gemeint sind, und diese zeigen beide nur die Sichtlinienkomponente der Magnetfelder. Im Innern des Flecks sollten die Magnetfelder ungefähr parallel zur Sichtlinie sein und somit die stärk- 


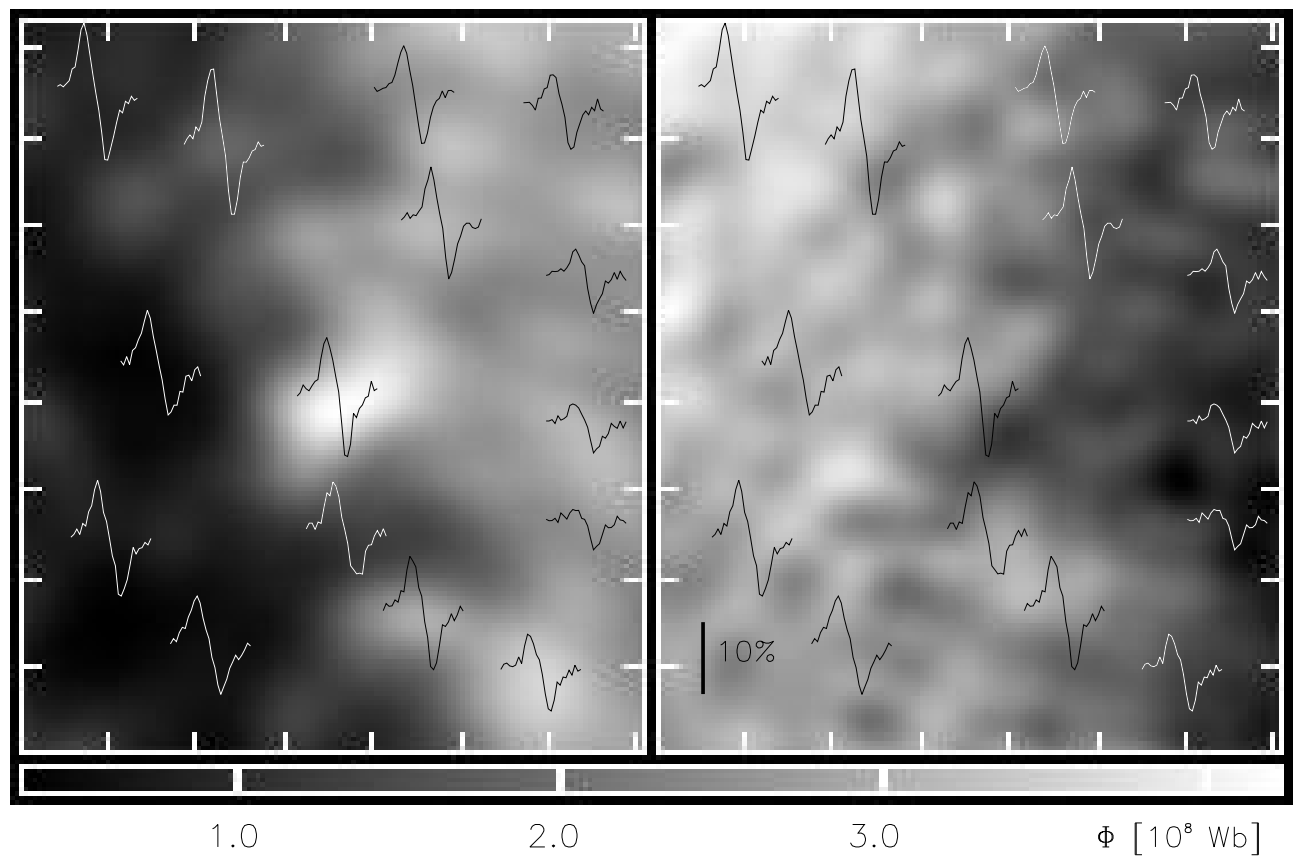

Abbildung 6.29: Ausschnitt ii aus Abb. 6.27 mit Aufhellung am rechten unteren Rand des Sonnenflecks. Links die Speckle-Rekonstruktion, rechts die Magnetfeldkarte. Der Abstand der Strichmarken entspricht $0,5^{\prime \prime}$.

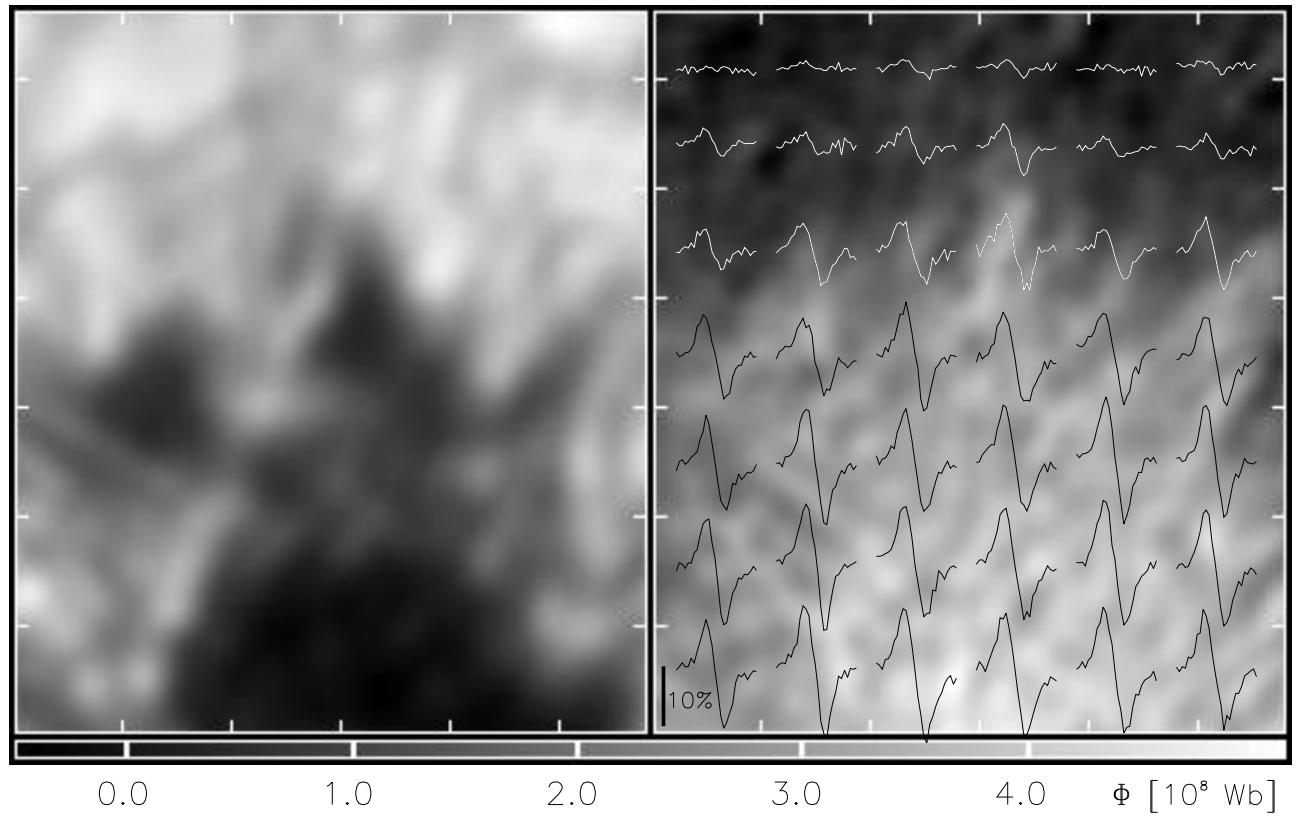

Abbildung 6.30: Ausschnitt iii aus Abb. 6.27 mit oberem Rand des Sonnenflecks. Links die Speckle-Rekonstruktion, rechts Magnetfeldkarte. Der Abstand der Strichmarken entspricht 1". 


\section{Ergebnisse}

sten Signale erzeugen, während mit zunehmendem Abstand vom Mittelpunkt des Flecks die Inklinationen zur Sonnenoberfläche und damit zur Sichtlinie im Mittel immer stärker werden sollten. Schon aus diesem Grunde würde man eine Abnahme dieser magnetischen Signale zum Rand des Flecks hin erwarten.

Zusammenfassend kann über den kleinen Sonnenfleck gesagt werden, dass sich seine magnetischen Eigenschaften nicht wesentlich von denen der vorher besprochenen Poren unterscheiden. Eine genauere Untersuchung z. B. der Feldinkliniation steht aber in diesem Zusammenhang noch aus. Der Fleck ist eingebettet in weitgehend ruhige Granulation, die lediglich vereinzelte schwache magnetische Strukturen zeigt, wie sie auch in Kap. 6.1 beschrieben wurden. Da dieser Fleck mit der Porengruppe aus Kap. 6.2 eine kleine, bipolare aktive Region bildete (vgl. Kap. 4.2), und in dieser ebenfalls eine vorwiegend einheitliche magnetische Polarität (entgegengesetzt zu der im Fleck) beobachtet wurde, kommt man zu dem naheliegenden Schluss, dass der dem Fleck entspringende magnetische Fluss nicht in der umgebenden Granulation abtaucht, sondern zum überwiegenden Teil mit den zugehörigen Poren verbunden ist. 


\section{Zusammenfassung und Ausblick}

Der vorliegenden Arbeit lagen unterschiedliche Aufgabenstellungen zu Grunde, die im Folgenden noch einmal kurz umrissen werden sollen. Weiterhin soll darauf eingegangen werden, inwieweit die gesteckten Ziele erreicht wurden und welches die zukünftigen Möglichkeiten und Ziele der jeweiligen Problemstellungen sein könnten.

\subsection{Einbau eines zweiten FPIs in das Spektro-Polarimeter}

Das „Göttinger“ zweidimensionale Spektro-Polarimeter am Vakuum-Turm-Teleskop (VTT) des Observatorio del Teide auf Teneriffa sollte durch den Einsatz eines zweiten Fabry-Perot-Interferometers modernisiert werden. Ziel war es, die Transmission der optischen Anlage soweit zu erhöhen, dass die Aufnahme schmalbandiger Filtergramme bei Belichtungszeiten von einigen Millisekunden möglich wird. Dieses Vorhaben ist gelungen. Nach erfolglosen Versuchen mit einem vorhandenen Fabry-Perot-Interferometer, das aber den hohen Qualitätsansprüchen nicht genügte, wurde ein Gerät der Firma „Queensgate Instruments Ltd.“ angeschafft. Dieses wurde erfolgreich in den Aufbau des 2d-Spektro-Polarimeters integriert (Kap. 3.2). Optische Probleme, wie das Auftreten von Reflexen zwischen den beiden FPIs, konnten behoben werden. Die elektronische Steuerung des Beobachtungsablaufs wurde entsprechend angepasst, und geeignete Verfahrensweisen für die Benutzung der neuen Geräte wurden entwickelt und in Form einer „Bedienungsanleitung“ den zukünftigen NutzerInnen der Anlage zur Verfügung gestellt. Es ist nun möglich, bei einer spektralen Bandbreite der Größenordnung $\Delta \lambda=5 \mathrm{pm}$ CCD-Aufnahmen mit einer Belichtungszeit $\Delta t$ von $10-30 \mathrm{~ms}$ zu erhalten (unter normalen Beobachtungsbedingungen). Damit ist auf der einen Seite gewährleistet, dass die Aufnahmen einen spektroskopischen Nutzen haben, z. B. indem mit aufeinander folgenden Aufnahmen das Linienprofil einer Spektrallinie abgetastet wird, auf der anderen Seite ermöglicht die kurze Belichtungszeit die Anwendung von Bildrekonstruktionsmethoden auf die Daten, um die störenden Einflüsse atmosphärischen Seeings nachträglich beseitigen zu können.

Sicherlich befindet man sich dabei sowohl bei der spektralen Bandbreite wie auch bei 


\section{Zusammenfassung und Ausblick}

der Belichtungszeit an den Grenzen der sinnvollen Bereiche. Noch schmalbandigere Aufnahmen wären wünschenswert, um feinere spektrale Details von Linienprofilen untersuchen zu können, oder einfach um Spektrallinien von geringerer Äquivalentbreite der Untersuchung zugänglich zu machen. Weiterhin könnte mit schmalbandigeren Aufnahmen eine genauere Untersuchung der Höhenschichtung der Sonnenatmosphäre gelingen, weil das Licht aus den verschiedenen Bereichen der Spektrallinien aus verschiedenen Atmosphärenschichten stammt (Einzelheiten s. Krieg et al. (1999b)). Bezüglich der Belichtungszeiten liegt man mit den genannten Werten ebenfalls an der Grenze dessen, innerhalb der man von „eingefrorenem Seeing“ sprechen kann, so dass sich die momentane OTF und damit das beobachtete Bild während der Belichtungszeit nicht wesentlich ändern. Gute Bildrekonstruktionsergebnisse sind bei gutem Seeing und geeigneten atmosphärischen Bedingungen durchaus noch bei Belichtungszeiten von 60 ms zu erhalten. Gerade aber im kurzwelligeren Bereich des sichtbaren Spektrums, in dem die typischen Korrelationszeiten geringer sind, wäre man mit Belichtungszeiten von 10 ms oder weniger auf der sicheren Seite.

Verbesserungen in diesem Bereich wären auf mehrerlei Weise zu erreichen:

- Die Gesamt-Transmission des optischen Aufbaus kann weiter optimiert werden. Insbesondere bei den als Vorfiltern verwendeten Interferenzfiltern (IF 2 in Abb. 3.2) besteht die Möglichkeit, Filter mit höherer Transmission zu erwerben, was für einige Spektrallinien mittlerweile erfolgt ist. Filter mit einer Maximaltransmission von über $60 \%$ sind im Handel erhältlich (inklusive Blockung für infrarotes Licht).

- Das schmalbandige FPI 2 könnte durch ein moderneres FPI ersetzt werden, vorzugsweise durch ein gleichartiges Gerät wie FPI1 (natürlich mit anderem Plattenabstand). Dieses würde eine etwas höhere Transmission aufweisen. Gleichzeitig wären dann durch die automatische Stabilisierung die Drift-Probleme von FPI 2, die eine genaue Wellenlängenfestlegung des Scans erschweren, behoben, ebenso wie die Probleme mit dem Absinken der effektiven Finesse $\mathcal{F}_{\text {eff }}$ während der Aufnahmeserien (Kap. 3.2).

- Neue Detektoren in Form neuer CCD-Kameras könnten eine höhere Quanteneffizienz haben, d. h. bei gleicher vorhandener Lichtmenge würde ein höherer Anteil der Photonen detektiert. Der Einsatz von Bildverstärkern ist allerdings nicht ratsam.

- Durch den Einsatz dieser oder einer ähnlichen Beobachtungsanlage an einem größeren Teleskop könnte, bei gleicher räumlicher Auflösung, mehr Licht pro Beobachtungselement und Spektralbereich zur Verfügung stehen. Bei dem dann nötigen räumlichen „undersampling“ der Daten könnten allerdings Probleme bei der Fourierdarstellung der Daten auftreten (,aliasing“ usw.).

All diese Verbesserungen könnten einerseits dazu führen, schmalbandigere Aufnahmen zu erhalten, oder die Belichtungszeiten zu verkürzen bzw. auch bei ungünstigeren Be- 
dingungen Daten aufzunehmen, z. B. wenn die Transparenz der Erdatmosphäre durch Staub verringert ist, oder bei Beobachtungen am Sonnenrand oder in Sonnenflecken. Ebenso könnte dadurch aber auch unter Beibehaltung der oben genannten Werte das Signal-zu-Rausch-Verhältnis (SNR) der Daten verbessert werden, was die nachfolgende Bildrekonstruktion erleichtern würde.

\subsection{Bildrekonstruktion und Phase Diversity Methode}

Die aufzunehmenden Daten sollten durch Bildrekonstruktionsmethoden von den störenden Einflüssen des atmosphärischen Seeings befreit werden, um hochwertiges Datenmaterial mit einer räumlichen Auflösung besser als 1" zu erhalten, das dann der spektroskopischen Analyse zugeführt werden sollte. Insbesondere sollte versucht werden, die in der Entwicklung befindliche Phase Diversity Methode zu testen und das Verfahren gegebenenfalls in der Göttinger Arbeitsgruppe „Experimentelle Sonnenphysik“ zu etablieren (Kap. 5.1.6). Dieses Vorhaben ist nur zum Teil gelungen. Durch die Integration optischer Komponenten und einer weiteren CCD-Kamera in die Beobachtungsanlage wurden die experimentellen Voraussetzungen geschaffen, für die Anwendung der Phase Diversity Methode geeignete Daten aufzunehmen. Die Auswertung der Daten und die Anwendung der Methode brachten aber nur Teilerfolge. Mit einiger Mühe konnten einigermaßen zuverlässige Wellenfrontfehler und damit momentane Optische Transferfunktionen (OTFs) für die aufgenommenen Datenpaare berechnet werden. Die damit erhaltenen Bildrekonstruktionen, insbesondere der schmalbandigen Aufnahmen, erreichten jedoch nicht die hohen Qualitätsansprüche.

Deshalb wurden weitere Bildverarbeitungs- und Rekonstruktions-Methoden ausprobiert. Das Ergebnis der umfangreichen Testreihen ist, dass eine auf der Speckle-Interferometrie basierende Rekonstruktions-Methode die besten Ergebnisse liefert (Kap. 5.1.7). Die an den einzelnen spektralen Positionen eines Scans berechneten Einzelbilder zeigen eine große Vielfalt von Feinstrukturen bis hinunter zu einer Größe von 0,4" (ca. $300 \mathrm{~km}$ auf der Sonnenoberfläche) und gute Kontraste. Ebensolche räumliche Auflösung wird in den daraus berechneten physikalischen Datenfeldern erreicht, also z. B. in Magnetfluss- und Geschwindigkeitskarten.

Dieses Ergebnis stellt einen erheblichen Gewinn gegenüber bisherigen Beobachtungsverfahren ohne Bildrekonstruktion dar, ebenso wie gegenüber eindimensionalen spektroskopischen Analysen mit klassischen Spaltspektrografen. Diese sind dem hier beschriebenen Beobachtungsverfahren allerdings in der spektralen Auflösung meistens überlegen.

Je nach Anwendungsgebiet, den technischen Voraussetzungen und Möglichkeiten und nach dem vertretbaren Aufwand können jedoch auch die anderen in Kap. 5 beschriebenen Datenverarbeitungsmethoden sinnvoll einsetzbar sein. Für alle Methoden gilt jedoch gleichermaßen, dass die besten Ergebnisse mit Daten erreicht werden, die ein hohes SNR aufweisen, und die, noch wichtiger, bei exzellentem Seeing aufgenommen worden sind. 
Deshalb gilt es, jede Möglichkeit zu nutzen, durch geeignete konstruktive Maßnahmen unnötiges Seeing zu vermeiden, sowohl außerhalb des Teleskops (Gebäude, Umgebung) wie auch innerhalb (Evakuierung, Luftzug). Auch Maßnahmen wie der Einsatz adaptiver Optik zur direkten Verbesserung der Seeing-Qualität während der Datenaufnahme könnte die spätere Bildrekonstruktion erleichtern. Weiterhin erhält man aus einer großen Auswahl von Bildern am ehesten einzelne, hochaufgelöste Aufnahmen mit hohem Informationsgehalt über kleinste Strukturen. Durch Modernisierung der Detektoren könnten also Möglichkeiten geschaffen werden, in kurzer Zeit mehr Bilder aufzunehmen, aus denen dann die besten (oder alle) für die weitere Bildrekonstruktion ausgewählt werden. Ein erster Schritt in dieser Richtung ist durch den Einbau neuer Steuer-PCs in das Spektro-Polarimeter bereits getan, so dass in Zukunft umfangreichere Datenmengen aufgenommen werden können. Der Einsatz neuer, schnellerer CCD-Kameras könnte auch in diesem Bereich weitere Verbesserungen bringen.

\subsection{Untersuchung magnetischer Strukturen}

Die mit dem „Göttinger“ zweidimensionalen Spektro-Polarimeter aufgenommenen und mit der Speckle-Methode rekonstruierten Datensätze sind ein hervorragendes Ausgangsmaterial, kleinste Strukturen auf der Sonnenoberfläche zu untersuchen, und dabei die spektroskopischen Informationen, also die Linienprofile $I(\lambda)$ und die polarimetrischen Profile $V(\lambda)$, zur Bestimmung physikalischer Größen wie Magnetfeldern oder Geschwindigkeiten zu nutzen. Dazu mussten zunächst Methoden zur Bestimmung dieser Größen (Berechnung von Stokes-Profilen, Linienschwerpunktsmethode, u. a.) sorgfältig an die vorliegenden Datensätze angepasst werden (Kap. 5.2). Durch weitere Maßnahmen (Mittelung, Glättung von Profilen, Korrekturen 2. Ordnung) ist es gelungen, bei einer räumlichen Auflösung von 0, $4^{\prime \prime}-0,5^{\prime \prime}$ magnetische Stokes-V-Signale bis hinunter zu 0,5\% $1 \%$ nachzuweisen, bei der Linienschwerpunktsmethode liegt die Nachweisgrenze bei etwa $5 \cdot 10^{7} \mathrm{~Wb}$ in einem Auflösungselement (Pixel), das auf der Sonnenoberfläche einem Gebiet von $73 \mathrm{~km} \times 73 \mathrm{~km}$ entspricht.

Hier zeigen sich Stärken und Schwächen der vorliegenden Daten und Ergebnisse: Im Vergleich zu ähnlichen Untersuchungen (z. B. Sigwarth (1999)] konnte eine hervorragende, sonst kaum erreichte räumliche Auflösung erzielt werden, dagegen liegt die magnetische Empfindlichkeit noch deutlich unter der anderer Analysen. Hier könnten aber die genannten Verbesserungsmöglichkeiten noch erhebliche Fortschritte bringen.

Während mehrerer Beobachtungsaufenthalte konnten zahlreiche Datensätze von verschiedenen Objekten der Sonnenoberfläche gewonnen werden. Von diesen sind allerdings nur die Datensätze mit dem besten Seeing in die vorliegende Arbeit eingeflossen. Sie zeigen alle Stufen magnetischer Aktivität von ruhiger Granulation über Plage-Gebiete und Poren bis zu einem kleinen Sonnenfleck. Alle beobachteten Objekte befinden sich ungefähr in der Mitte der Sonnenscheibe, so dass in den meisten Fällen davon ausgegangen 


\subsection{Untersuchung magnetischer Strukturen}

werden kann, dass die magnetischen Feldlinien etwa parallel zur Beobachtungsrichtung sind und man es deshalb vorwiegend mit longitudinalem Zeeman-Effekt zu tun hat.

Sorgfältige Inspektionen einzelner Gebiete der Bildausschnitte, aber auch statistische Untersuchungen vieler Messpunkte, brachten umfangreiche Erkenntnisse über Eigenschaften und Dynamik kleinskaliger magnetischer Strukturen:

- Auch scheinbar ruhige, unmagnetische Gebiete der Sonnenoberfläche enthalten in der Granulation zahlreiche schwach magnetische Strukturen, aber vereinzelt auch etwas stärkere „magnetische Knoten“. Diese können dann mit Fackelpunkten verbunden sein, die im Kontinuumslicht vor allen Dingen durch ihre geringen Ausmaße $\left(<0,5^{\prime \prime}\right)$ und ihre Lage im Intergranulum auffallen, in Linienkernbildern dagegen durch ihre überdurchschnittliche Intensität. Diese etwas stärkeren Strukturen könnten Teile des chromosphärischen Netzwerks sein. Dies bestätigt Ergebnisse von Sigwarth (1999).

- Gerade die schwachen magnetischen Strukturen zeigen in dieser Untersuchung Durchmesser von wenigen 100 km. Bei Berücksichtigung der räumlichen Auflösung der Daten muss deshalb davon ausgegangen werden, dass die wahren Durchmesser der Strukturen noch kleiner sind.

- Bei schwachen magnetischen Strukturen innerhalb der Granulation kommt es häufig vor, dass in sehr geringen Abständen bis hinunter zu 0, 4" Strukturen entgegengesetzter Polarität nebeneinander auftreten.

- Bei stärker magnetischen Gebieten (Plages) konnten keine gemischten Polaritäten innerhalb sehr kurzer Distanzen gemessen werden.

- Bei stärkeren magnetischen Strukturen wie Poren oder dem kleinen Sonnenfleck zeigt das magnetische Signal im Gegensatz zur Intensität nur wenig innere Struktur, d. h. der magnetische Fluss verteilt sich recht gleichmäßig über die ganze Struktur. Nichtsdestotrotz können an den Rändern solcher Gebiete starke Gradienten des magnetischen Flusses auftreten, d. h. innerhalb von Distanzen $<1^{\prime \prime}$ können die magnetischen Flusswerte von null auf die Maximalwerte innerhalb der Struktur ansteigen. Insbesondere konnten im Innern von Poren keine feldfreien Gebiete gefunden werden.

- Das Magnetfeld bei Poren reicht häufig über die im Kontinuumslicht als Verdunklung sichtbare Pore hinaus. Die gemessenen Feldstärken von bis zu 0,22 T bestätigen bisherige Messungen (z. B. Sütterlin (1996)]).

- Magnetische Strukturen zeigen im Vergleich zur Granulation eine charakteristische statistische Intensitätsverteilung. Im Kontinuumslicht können sich schwache Strukturen $\left(0,5-1,5 \cdot 10^{8} \mathrm{~Wb}\right.$ in einem Pixel) sowohl im dunklen Intergranulum, wie auch in mäßig hellen Granulen oder an deren Randgebieten befinden. 
Nur das Innere heller Granulen zeigt eine deutliche Unterrepräsentation von Magnetfeldern. Etwas stärkere Strukturen $\left(1,5-2,5 \cdot 10^{8} \mathrm{~Wb}\right.$ in einem Pixel) zeigen häufig etwas erhöhte Intensität (Fackelpunkte). Bei noch stärkeren Magnetfeldern $\left(>2,5 \cdot 10^{8} \mathrm{~Wb}\right.$ in einem Pixel) werden die Strukturen aber zunehmend dunkler und gehen fließend in Poren und Flecken über.

- Im Licht des Linienkerns der FeI-Linie bei 630, $25 \mathrm{~nm}$ ist dagegen eine sofortige Erhöhung der Intensität mit steigendem Magnetfeld zu beobachten. Auch hier werden aber die Strukturen bei Flüssen oberhalb $2,5 \cdot 10^{8} \mathrm{~Wb}$ in einem Pixel dunkel, so dass Poren und Flecken sichtbar werden.

- Mit der Linienschwerpunktsmethode können auch Geschwindigkeiten gemessen werden. Dabei ergeben sich die Sichtlinienkomponenten der Materialbewegung aus ihren Dopplerverschiebungen, und zwar gemittelt über den gesamten Bereich der von der Spektrallinie abgetasteten Höhenschichten. Für unmagnetische Gebiete ergeben sich Auf- und Abströmungen von bis zu 1,5km/s. Für Strukturen mit Magnetflüssen von 0,5-1,5 $\cdot 10^{8} \mathrm{~Wb}$ in einem Pixel ergibt sich eine Abnahme der maximalen Aufströmungen auf etwa $0,3 \mathrm{~km} / \mathrm{s}$, während die maximalen Abströmungen unverändert bleiben. Bei noch stärkeren Feldern $\left(>1,5 \cdot 10^{8} \mathrm{~Wb}\right.$ in einem Pixel) findet man eine konstante Geschwindigkeitsverteilung zwischen Abströmungen von $1,5 \mathrm{~km} / \mathrm{s}$ und Aufströmungen von $0,3 \mathrm{~km} / \mathrm{s}$.

- Die Dynamik der Strukturen hängt deutlich mit ihrer magnetischen Stärke zusammen. Die schwächsten gemessenen Magnetfelder haben die geringsten Lebensdauern von nur wenigen Minuten oder verändern ebensoschnell ihre Form und Lage. Häufig ist schon bei aufeinander folgenden Datensätzen im zeitlichen Abstand von $74 \mathrm{~s}$ nicht mehr klar erkennbar, welche Strukturen einander zugeordnet werden können. Dabei scheint die Dynamik der magnetischen Strukturen von der Dynamik der Granulation bestimmt zu sein, d. h. die magnetischen Strukturen werden von der Granulation umhergestoßen und verschoben. Kleine Elemente können sich spalten oder verschmelzen.

- Stärkere magnetische Gebilde wie Plages oder Poren zeigen eine eher geringe Dynamik, d. h. sie verändern sich nach Stärke und Form der Magnetfelder wenig, jedenfalls während der in dieser Untersuchung vorliegenden Messreihen von insgesamt ca. 25 Minuten Länge.

- Mit abnehmendem magnetischen Fluss nimmt die Streuung anderer physikalischer Größen wie Geschwindigkeiten und Intensitäten um ihren Mittelwert zu. Dies stützt Beobachtungen von Grossmann-Doerth et al. (1996) und Sigwarth (1999) und Simulationen von Steiner et al. (1998),

- In einem Gebiet ruhiger Photosphäre konnte in einem Fall das kurzzeitige Auftauchen einer kleinen, bipolaren Struktur beobachtet werden. Die beiden Komponen- 


\subsection{Untersuchung magnetischer Strukturen}

ten zeigen Flüsse von $\pm 1,5 \cdot 10^{8} \mathrm{~Wb}$ in einem Pixel und haben einen Abstand von $0,5^{\prime \prime}$.

- In einem anderen Gebiet ruhiger Photosphäre konnte in einem Fall die Konzentration und Verstärkung des magnetischen Flusses bei gleichzeitigem Auftreten einer kräftigen Abströmung von 1,4 km/s beobachtet werden. Möglicherweise wird hier der z. B. bei Stix (1989) beschriebene „konvektive Kollaps“ beobachtet.

- In der Umgebung des kleinen Sonnenflecks konnte kein nennenswerter magnetischer Fluss gefunden werden, der als Abtauchen des aus dem Fleck austretenden Flusses interpretiert werden könnte. Dabei muss allerdings erwähnt werden, dass dieser Fleck als Teil einer bipolaren Gruppe mit einem benachbarten Plage-Gebiet in Verbindung stand.

Bei den vorliegenden Untersuchungen konnte die Form der Stokes-V-Profile dazu verwendet werden, magnetische Strukturen aufzuspüren und halbquantitativ ihre ungefähre Stärke zu bestimmen. Leider waren jedoch die genauen Formen der Profile, also insbesondere pathologische Formen und Asymmetrien, nicht so zuverlässig zu bestimmen, dass aus ihnen physikalische Schlussfolgerungen abgeleitet werden sollten. Bei zukünftigen Untersuchungen sollte aber versucht werden, mit höherer spektraler Auflösung und einer zuverlässigen Abschätzung der instrumentellen Polarisation größere Sicherheit bei der Bestimmung der Linienprofile und der Asymmetrien der Stokes-V-Profile zu erhalten. Vergleiche mit anderen Untersuchungen (Sigwarth (1999), Martínez Pillet et al. (1990]) und theoretischen Überlegungen (Sánchez Almeida et al. (1996) u. a.) wären dann möglich. Ebenso könnten dann mittels Inversions-Rechnungen viele weitere physikalische Parameter aus den Linienprofilen berechnet werden (Bellot Rubio et al. (2000), Ritter (2001), u. a.) Auch könnten dann genauere Geschwindigkeitsmessungen anhand des Nulldurchgangs der Stokes-V-Profile bestimmt werden.

Weiterhin wäre es wünschenswert, eine zuverlässige Abschätzung des Streulichts zu erhalten, das die Messungen der Stärke des magnetischen Flusses und der Stokes-VAmplituden verfälscht. Dazu könnten Vergleichsmessungen mit anderen Instrumenten durchgeführt werden, deren Streulichtanteile bekannt sind. Dies würde zuverlässigere quantitative Angaben für diese Größen liefern. Hierzu wären allerdings zusätzlich noch genauere Angaben über die instrumentelle Polarisation vonnöten.

Durch den Einbau neuer CCD-Kameras als Detektoren könnten mehrere Ziele erreicht werden:

- Größere Bildfelder würden die Untersuchung weiträumigerer Gebiete ermöglichen, statistische Aussagen über das Verhalten oder die Eigenschaften bestimmter Strukturen würden sicherer werden.

- Die einzelnen Datensätze könnten in schnellerer zeitlicher Folge aufgenommen werden, so dass die Entwicklung gerade der schwächsten magnetischen Strukturen 
besser verfolgt werden könnte. Auch Messungen von Oszillationen würden dadurch erleichtert.

- Pro Wellenlängenposition könnten evtl. mehr Einzelbilder aufgenommen werden, was die Möglichkeiten der Bildrekonstruktion erweitern würde.

- Eine höhere Empfindlichkeit der neuen Detektoren hätte, wie oben schon beschrieben, Vorteile in Bezug auf spektrale Bandbreite, Belichtungszeit und SNR der Daten.

Durch Untersuchung anderer Spektrallinien könnten viele weitere Erkenntnisse gewonnen werden. Beispielsweise könnten durch Beobachtung einer tief in der Photosphäre entstehenden, magnetisch sensitiven Spektrallinie andere Korrelationen zwischen dem Auftreten magnetischer Felder und der Kontinuumsintensität gefunden werden.

Als lohnenswerte Beobachtungsobjekte kommen natürlich weiterhin die verschiedensten magnetischen oder (scheinbar) unmagnetischen Strukturen der Sonnenoberfläche in Frage, allen voran die ruhige Photosphäre mit ihren vielfältigen magnetischen Signaturen, die schon in der vorliegenden Untersuchung in großer Zahl gefunden wurden. Speziell könnte sich die Entwicklung von Magnetfeldern innerhalb oder in der Umgebung explodierender Granulen als interessant erweisen. Weitere und vielleicht eindeutigere Hinweise auf Phänomene wie den „konvektiven Kollaps“ (Kap. 6.1.2) sind wünschenswert, ebenso wie noch genauere Beobachtungen der Interaktion schwacher Magnetfelder mit der umliegenden Granulation.

Zu guter Letzt muss festgestellt werden, dass durch den Einsatz dieser oder ähnlicher Beobachtungstechniken an einem größeren Teleskop zahlreiche Untersuchungen mit noch besseren Ergebnissen durchgeführt werden könnten und sich darüber hinaus viele neue Möglichkeiten und Untersuchungsobjekte erschließen würden. Es darf deshalb auf die baldige Realisierung der Pläne für ein 1,5 m-Teleskop (GREGOR) am „Observatorio del Teide" auf Teneriffa gehofft werden. 


\section{Literaturverzeichnis}

[Acton (1996)] D. S. Acton, private Mitteilung, 1996

[Atherton et al. (1981)] P. D. Atherton, N. K. Reay, J. Ring, Tunable Fabry-Perot filters, Optical Engineering 20, Nr. 6, 806-814, 1981

[Beckers \& Schröter (1968)] J. M. Beckers, E. H. Schröter, The intensity, velocity and magnetic structure of a sunspot region, Solar Physics 4, 142-164, 1968

[Bellot Rubio et al. (2000)] L. R. Bellot Rubio, B. Ruiz Cobo, M. Collados, Inversion of Stokes profiles from solar magnetic elements, The Astrophysical Journal 535, 475-488, 2000

[Bendlin (1993)] C. Bendlin, Hochauflösende zweidimensionale Spektroskopie der solaren Granulation mit einem Fabry-Perot-Interferometer, Dissertation Universität Göttingen, 1993

[Bendlin \& Volkmer (1995)] C. Bendlin, R. Volkmer, The two-dimensional spectrometer in the German Vacuum Tower Telescope/Tenerife, Astronomy \& Astrophysics Supplement Series 112, 371-382, 1995

[Bianda et al. (1998)] M. Bianda, S. K. Solanki, J. O. Stenflo, Hanle depolarisation in the solar chromosphere, Astronomy \& Astrophysics 331, 760-770, 1998

[Bianda et al. (1998a)] M. Bianda, J. O. Stenflo, S. K. Solanki, Hanle diagnostics of solar magnetic fields: The Sr II 4078 A line, Astronomy \& Astrophysics 337, 565-578, 1998

[Born \& Wolf (1971)] M. Born, E. Wolf, Principles of optics, Pergamon Press, 1971

[Brault \& White (1971)] J. W. Brault, O. R. White, The analysis and restoration of astronomical data via the fast Fourier transform, Astronomy \& Astrophysics 13, 169-189, 1971

[Brault \& Neckel (1987)] J. W. Brault, H. Neckel, Spectral atlas of Solar absolute disk-averaged and disk-center intensities from 3290 to $12510 \AA$, 1987, zitiert in H. Neckel, Solar Physics 184, 421-422, 1999 
[de Boer (1993)] C. R. de Boer, Speckle-Interferometrie und ihre Anwendung auf die Sonnenbeobachtung, Dissertation Universität Göttingen, Verlag Shaker Aachen, 1993

[Degenhardt \& Wiehr (1991)] D. Degenhardt, E. Wiehr, Spatial variation of the magnetic field inclination in a sunspot penumbra, Astronomy \& Astrophysics 252, 821-826, 1991

[Denker (1996)] C. Denker, Untersuchung der Feinstruktur von Sonnenflecken mit Methoden der Speckle-Interferometrie, Dissertation Universität Göttingen, Aktiv Druck Verlag Göttingen, 1996

[Faurobert-Scholl (1993)] M. Faurobert-Scholl, Investigation of microturbulent magnetic fields in the solar photosphere by their Hanle effect in the $\mathrm{Sr} \mathrm{I} 4607 \AA$ line, Astronomy \& Astrophysics 268, 765-774, 1993

[Fried (1966)] D. L. Fried, Optical resolution through a randomly inhomogeneous medium for very long and very short exposures, Journal of the Optical Society of America 56, 1372-1379, 1966

[Gonsalves \& Chidlaw (1979)] R. A. Gonsalves, R. Chidlaw, Wavefront sensing by phase retrieval, in Applications of digital image processing III, A. G. Tescher (Ed.), Proc. SPIE, 32-39, 1979

[Gonsalves (1982)] R. A. Gonsalves, Phase retrieval and diversity in adaptive optics, Optical Engineering 21, Nr. 5, 829-832, 1982

[Grossmann-Doerth et al. (1996)] U. Grossmann-Doerth, C. U. Keller, M. Schüssler, Observations of the quiet Sun's magnetic field, Astronomy \& Astrophysics 315, 610-617, 1996

[Grossmann-Doerth et al. (1998)] U. Grossmann-Doerth, M. Schüssler, O. Steiner, Convective intensification of solar surface magnetic fields: results of numerical experiments, Astronomy \& Astrophysics 337, 928-939, 1998

[Hirzberger et al. (2001)] J. Hirzberger, M. Koschinsky, F. Kneer, C. Ritter, High resolution 2D-spectroscopy of granular dynamics, Astronomy \& Astrophysics, im Druck, 2001

[Hofmann (2000)] A. Hofmann, Astrophysikalisches Institut Potsdam (AIP), private Mitteilung, 2000

[Karo \& Schneidermann (1978)] D. P. Karo, A. M. Schneidermann, Speckle interferometry at finite spectral bandwidths and exposure times, Journal of the Optical Society of America 68, Nr. 4, 480-485, 1978 
[Keller \& v. d. Lühe (1992)] C. U. Keller, O. von der Lühe, Solar speckle polarimetry, Astronomy \& Astrophysics 261, 321-328, 1992

[Keller et al. (1994)] C. U. Keller, F.-L. Deubner, U. Egger, B. Fleck, H. P. Povel, On the strength of solar intra-network fields, Astronomy \& Astrophysics 286, 626-634, 1994

[Kentischer et al. (1998)] T. J. Kentischer, W. Schmidt, M. Sigwarth, M. von Uexküll, TESOS, a double Fabry-Perot instrument for solar spectroscopy, Astronomy \& Astrophysics 340, 569-578, 1998

[Knox \& Thompson (1974)] K. T. Knox, B. J. Thompson, Recovery of images from atmospherically degraded short-exposure photographs, The Astrophysical Journal 193, L 45-L 48, 1974

[Koschinsky (1995)] M. Koschinsky, Untersuchung der Polarisationseigenschaften optischer Geräte, Diplomarbeit Universität Göttingen, 1995

[Koschinsky et al. (1999)] M. Koschinsky, J. Krieg, F. Kneer, C. Ritter, M. Wunnenberg, in High resolution solar physics: Theory, observations and techniques, T. R. Rimmele, K. S. Balasubramaniam, R. R. Radick (Eds.), ASP Conference Series 183, 349-356, 1999

[Koschinsky et al. (2001)] M. Koschinsky, F. Kneer, J. Hirzberger, Speckle spectropolarimetry of solar magnetic structures, Astronomy \& Astrophysics 365, 588-597, 2001

[Korff (1973)] D. Korff, Analysis of a method for obtaining near-diffraction-limited information in the presence of atmospheric turbulence, Journal of the Optical Society of America 63, 971-980, 1973

[Krieg (1999 a)] J. Krieg, Bildrekonstruktion zweidimensionaler Spektrogramme zur Untersuchung der solaren Granulation, Dissertation Universität Göttingen, Cuvillier Verlag Göttingen, 1999

[Krieg et al. (1999 b)] J. Krieg, M. Wunnenberg, F. Kneer, M. Koschinsky, C. Ritter, Height variation of the solar granulation, Astronomy \& Astrophysics 343, 983-989, 1999

[Krieg et al. (2000)] J. Krieg, F. Kneer, M. Koschinsky, C. Ritter, Granular velocities of the Sun from speckle interferometry, Astronomy \& Astrophysics 360, 1157-1162, 2000

[Labeyrie (1970)] A. Labeyrie, Attainment of diffraction limited resolution in large telescopes by Fourier analysing speckle patterns in star images, Astronomy \& Astrophysics 6, 85-87, 1970 
[Landi Degl'Innocenti (1992)] E. Landi Degl'Innocenti, in Solar Observations: Techniques and interpretation, First Canary Islands Winter School of Astrophysics, F. Sánchez, M. Collados, M. Vázquez, Eds., Cambridge University Press, 73-143, 1992

[Lin (1995)] H. Lin, On the distribution of the solar magnetic fields, The Astrophysical Journal 446, 421-430, 1995

[Lin \& Rimmele (1999)] H. Lin, T. Rimmele, The granular magnetic fields of the quiet sun, The Astrophysical Journal 514, 448-455, 1999

[Löfdahl (1996)] M. G. Löfdahl, Phase diversity wavefront sensing and image restoration applied to high-resolution solar observations, Dissertation Universität Stockholm, 1996

[Mack et al. (1963)] J. E. Mack, D. P. McNutt, F. L. Roesler, R. Chabbal, The PEPSIOS purely interferometric high-resolution scanning spectrometer. I. The Pilot Model, Applied Optics 2, Nr. 9, 873-884, 1963

[Martínez Pillet et al. (1997)] V. Martínez Pillet, B. W. Lites, A. Skumanich, Active region magnetic fields. I. Plage Fields, The Astrophysical Journal 474, 810842,1997

[Navarro (1999)] D. Héctor Socas Navarro, NLTE inversions of spectral lines and Stokes profiles, Dissertation, IAC, Universidad de La Laguna, 1999

[Noll (1976)] R. Noll, Zernike polynomials and atmospheric turbulence, Journal of the Optical Society of America 66, 207-211, 1976

[Osherovich (1982)] V. A. Osherovich, A new magneto-hydrostatic theory of sunspots, Solar Physics 77, 63-68, 1982

[Rees \& Semel (1979)] D. E. Rees, M. D. Semel, Line formation in an unresolved magnetic element: a test of the centre of gravity method, Astronomy \& Astrophysics 74, 1-5, 1979

[Ritter (2001)] C. Ritter, Untersuchung polarer Fackeln mittels Bildrekonstruktion und Inversionsrechnungen, Dissertation Universität Göttingen, 2001

[Rust (1991)] D. M. Rust, Etalon Filters, Technical Report No. 50, LEST Foundation, ISSN 0800-7780, Institute of Theoretical Astrophysics, University of Oslo, 1991

[Sánchez Almeida \& Martínez Pillet (1994)] J. Sánchez Almeida, V. Martínez Pillet, The inclination of network magnetic fields, The Astrophysical Journal 424, 1014-1021, 1994 
[Sánchez Almeida et al. (1996)] J. Sánchez Almeida, E. Landi Degl'Innocenti, V. Martínez Pillet, B. W. Lites, Line asymmetries and the microstructure of photospheric magnetic fields, The Astrophysical Journal 466, 537-548, 1996

[Sánchez Almeida \& Lites (2000)] J. Sánchez Almeida, B. W. Lites, Physical properties of the solar magnetic photosphere under the MISMA hypothesis. II. Network and internetwork fields at the disk center, The Astrophysical Journal 532, 12151229,2000

[Semel (1967)] M. D. Semel, Contribution a L'Etude des Champs Magnétiques dans les Régions Actives Solaires, Annales D'Astrophysique 30, 513-551, 1967

[Sheeley (1967)] N. R. Sheeley, Observations of small-scale solar magnetic fields, Solar Physics 1, 171-179, 1967

[Shurcliff (1962)] W. A. Shurcliff, Polarized Light, Harvard University Press, Cambridge, Massachusetts, 1962

[Sigwarth (1999)] M. Sigwarth, Dynamik solarer Magnetfelder, Dissertation Universität Freiburg, 1999

[Smirnow (1990)] W. I. Smirnow, Lehrgang der höheren Mathematik, Teil I, 16. Auflage, 244-245, VEB Deutscher Verlag der Wissenschaften, 1990

[Soltau (1988)] D. Soltau, High resolution observations with the new German VTT on Tenerife, in "High spatial resolution solar observations“, Proceedings of the 10th NSO/SP Summer Workshop, Ed. O. v. d. Lühe, 3-9, 1988

[Soltau (1997)] D. Soltau, Stokes I and V observations of magnetic knots using bright Ca II K network points as tracers, Astronomy \& Astrophysics 317, 586-593, 1997

[Steiner et al. (1998)] O. Steiner, U. Grossmann-Doerth, M. Knölker, M. Schüssler, Dynamical interaction of solar magnetic elements and granular convection: results of a numerical simulation, The Astrophysical Journal 495, 468-484, 1998

[Steiner (1999)] O. Steiner, Small-scale magnetic flux tubes in the photosphere: a simulation perspective, in High resolution solar physics: theory, observations, and techniques, T. R. Rimmele, K. S. Balasubramaniam, R. R. Radick (Eds.), ASP conference series 183, 17-29, 1999

[Steiner (2000)] O. Steiner, The formation of asymmetric Stokes V profiles in the presence of a magnetopause, Solar Physics 196, 245-268, 2000

[Stenflo (1973)] J. O. Stenflo, Magnetic-field structure of the photospheric network, Solar Physics 32, 41-63, 1973 
[Stenflo et al. (1998)] J. O. Stenflo, C. U. Keller, A. Gandorfer, Differential Hanle effect and the spatial variation of turbulent magnetic fields on the sun, Astronomy \& Astrophysics 329, 319-328, 1998

[Stix (1989)] M. Stix, The Sun, Springer, Berlin, 1989

[Stolpe (1999)] F. Stolpe, Magnetfelder mit geringem magnetischen Fluß in der Sonnenatmosphäre, Dissertation Universität Göttingen, Cuvillier Verlag Göttingen, 1999

[Sütterlin (1996)] P. Sütterlin, Über die Struktur von solaren Poren, Dissertation Universität Freiburg, 1996

[Volkmer (1995)] R. Volkmer, Hochauflösende zweidimensionale Spektro-Polarimetrie solarer Magnetfelder, Dissertation Universität Göttingen, Verlag Shaker Aachen, 1995

[Vaughan (1989)] J. M. Vaughan, The Fabry-Perot interferometer, Adam Hilger, Bristol, 1989

[v. d. Lühe (1984)] O. von der Lühe, Estimating Fried's parameter from a time series of an arbitrary resolved object imaged through atmospheric turbulence, Journal of the Optical Society of America A 1, 510-519, 1984

[v. d. Lühe \& Kentischer (2000)] O. von der Lühe, T. J. Kentischer, High spatial resolution performance of a triple Fabry-Perot filtergraph, Astronomy \& Astrophysics Supplement Series 146, 499-506, 2000

[Weigelt (1977)] G. P. Weigelt, Modified astronomical speckle interferometry „speckle masking “, Optics communications 21, Nr. 1, 55-59, 1977 


\section{Verzeichnis der Internetadressen}

[1] National Solar Observatory (NSO), Kitt Peak

ftp://argo.tuc.noao.edu/SPMG_cdrom/SPMG_59/991022M1.Z

bzw. http://www.nso.noao.edu/nsokp/nsokp.html

[2] Mees Solar Observatory

http://www.solar.ifa.hawaii.edu/IVM/Archive/1999/AR8738_991022.2022_ivm.gif bzw. http://koa.ifa.hawaii.edu/

[3] $\operatorname{EIT}(\mathrm{SOHO})$

http://umbra.nascom.nasa.gov/eit/eit-catalog.html

und dort die Dateien efz19991022.130014 bzw. efz19991022.131851

[4] Big Bear Solar Observatory (BBSO)

ftp://ftp.bbso.njit.edu/pub/archive/1999/10/22/bbso_caiik_fr_19991022_155522.jpg ftp://ftp.bbso.njit.edu/pub/archive/1999/10/22/bbso_halph_fr_19991022_153536.jpg bzw. http://www.bbso.njit.edu/

Die Arbeit wurde finanziert von der Deutschen Forschungsgemeinschaft (DFG). 


\section{Lebenslauf}

- Name: Markus Koschinsky

- Geburt: 08. 09.1969 in Bad Lauterberg im Harz

- Staatsangehörigkeit: deutsch

- Schule:

- 1976-1980 Grundschule Scharzfeld

- 1980-1982 Orientierungstufe Herzberg

- 1982-1989 Ernst-Moritz-Arndt-Gymnasium Herzberg, Abitur

- Grundwehrdienst: 1989-1990 5. Panzergrenadierbataillon 12 in Osterode

- Studium:

- 1990-1995 Grundstudium der Physik an der Georg-August-Universität zu Göttingen, Diplom Juni 1995

- 1995-2001 Promotion im Rahmen eines DFG-Projekts, angestrebter Abschluss Frühjahr 2001

- Beruf:

- September-Dezember 2000 Anstellung als wissenschaftlicher Redakteur im „Institut für den wissenschaftlichen Film (IWF)“ in Göttingen im Rahmen des vom BMBF geförderten Projekts „IWF Digiclip“ (zeitlich befristet) 